\title{
Dewetting of Polymer Thin Films on Viscoelastic Substrates
}

\section{Dissertation}

zur Erlangung des mathematisch-naturwissenschaftlichen

Doktorgrades

"Doctor rerum naturalium"

der Georg-August-Universität Göttingen

vorgelegt von

\section{Konstantina Kostourou}

\author{
aus Athen, Griechenland
}

Göttingen, 2010 
Referent : Prof. Dr. Stephan Herminghaus

Koreferent : Prof. Dr. Christoph F. Schmidt

Tag der mündlichen Prüfung : 
To Daniel 



\section{Abstract}

In the present work, we study the wetting and dewetting phenomena on viscoelastic substrates, which are either planar or topographically structured.

The main focus has been given primarily to the planar substrates, where the dewetting behaviour of thin polystyrene (PS) films on viscoleastic substrates (cross-linked Polydimethylsiloxane - PDMS and molten Polymethylmethacrylate - PMMA) is explored. Our aim is to study how the viscoelastic properties of the substrate affect the dewetting dynamics as well as the equilibrium state of the overlying polymer thin film and to gain knowledge on the mechanisms that dominate the dewetting process.

Thus, we have determined the behaviour of the dewetting velocity as a function of time and as a function of the substrate's viscoelastic properties. The experimental results in both cases are in good agreement with previous theoretical predictions.

In particular, we are interested in the overall shape of the rim surrounding the growing hole, i.e. both the air/PS and PS/substrate interface. We demonstrate that the shape of the substrate deformation below the liquid rim changes dramatically depending on whether the substrate's elastic or viscous properties dominate.

Additionally, we study the shape of sessile PS droplets in equilibrium on the substrate. We are interested in the full shape of the droplet, i.e. the shape of the air/PS interface, the deformation at the dry side of the three phase contact line (TPCL) and the deformation below the droplet.

Finally, a preliminary study has been conducted regarding the wetting phenomena in rectangular grooves of PDMS and how these depend on the substrate wettability and geometry. Tuning independently these two parameters, the wetting dynamics is tailored accordingly, resulting in a different final order of the liquid morphologies. 



\section{Kurzzusammenfassung}

In der vorliegenden Arbeit werden Be- und Entnetzungsphänomene sowohl auf ebenen als auch auf topographisch strukturierten viskoelastischen Substraten untersucht.

Das Hauptaugenmerk richtet sich zunächst auf ebene Substrate, auf welchen das Entnetzungsverhalten von dünnen Polystyrolfilmen auf viskoelastischen Substraten (vernetzte Polydimethylsiloxane - PDMS und flüssige Polymethylmethacrylate - PMMA) erforscht wird. Ziel ist es dabei herauszufinden wie die viskoelastischen Eigenschaften den Gleichgewichtszustand des darüberliegenden Polymerdünnfilms als auch die Entnetzungsdynamik beeinflussen und Kenntnisse über die Mechanismen, die diesen Entnetzungsprozess dominieren, zu gewinnen.

Dazu wird das Verhalten der Entnetzungsgeschwindigkeit in Abhängigkeit der Zeit und der viskoelastischen Eigenschaften des Substrats bestimmt. In beiden Fällen zeigen die experimentellen Ergebnisse eine gute Übereinstimmung mit den theoretischen Vorhersagen.

Spezielles Interesse gilt der Form der Wulst, die das wachsende Loch im Flüssigkeitsfilm umgibt, d.h. sowohl die Form der Flüssig/Gas- als auch die der Flüssig/FestGrenzfläche. Wir können zeigen, dass die Deformation des Substrats unter der Flüssigkeitswulst davon abhängt, ob die elastischen oder die viskosen Eigenschaften des Substrats dominieren.

Desweiteren untersuchen wir die Form des auf dem Substrat sitzenden PS Tropfens im Gleichgewicht. Wieder interessiert uns sowohl die gesamte Form des Tropfens, d.h. die Form der Flüssig/Gas- und der Flüssig/Fest-, als auch die Deformation der DreiPhasen-Kontaktlinie.

Schließlich werden erste Experimente bezüglich Benetzungsphänomenen in rechteckigen Gräben aus PDMS durchgeführt. Die Benetzungsdynamik ist dabei sowohl für die Benetzbarkeit des Substrats, als auch für die davon unabhängige Grabengeometrie 
iv

charakteristisch und führt letztlich zu einer für diese Parameter spezifischen Ordung der Flüssigkeitsmorphologien. 


\section{Contents}

Abstract ................................ i

Kurzzusammenfassung . . . . . . . . . . . . . . . . iii

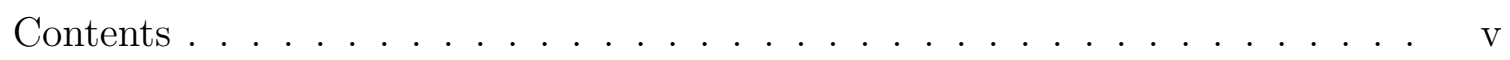

List of Figures . . . . . . . . . . . . . . . . . . ix

List of Symbols . . . . . . . . . . . . . . . . . . . . . xix

$\begin{array}{lr}\text { Introduction } & 1\end{array}$

$\begin{array}{lll}1 & \text { Theoretical Background } & 5\end{array}$

1.1 Fundamentals of wetting and dewetting . . . . . . . . . . . 5

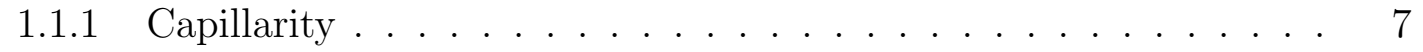

1.1 .2 Stability of homogeneous liquid films . . . . . . . . . . 12

$1.1 .3 \quad$ Moving liquid on a rigid substrate - Slippage . . . . . . . . . 16

1.2 Wetting of structured substrates $\ldots \ldots \ldots \ldots \ldots \ldots$

$1.2 .1 \quad$ Contact line pinning $\ldots \ldots \ldots \ldots \ldots$

1.3 Physical properties of polymers $\ldots \ldots \ldots \ldots \ldots$

$1.3 .1 \quad$ Physical states and transitions . . . . . . . . . . . . . . . . 21

1.3 .2 Rheology and mechanical properties of polymers . . . . . . . . 23

1.3 .3 Elastic modulus . . . . . . . . . . . . . . . . . . . . . . 25

1.3 .4 Viscoelasticity . . . . . . . . . . . . . . . . . 26

\begin{tabular}{|ll|l}
\hline 2 & Sample preparation and system characterisation & 39
\end{tabular}

$2.1 \quad$ Sample Preparation . . . . . . . . . . . . . . . . . . . . . . . . . . . . . . . 39

$2.1 .1 \quad$ Planar viscoelastic substrates . . . . . . . . . . . . . . 40

2.1 .2 Topographically structured viscoelastic substrates $\ldots \ldots$. . . . 46

2.2 Experimental techniques $\ldots \ldots \ldots \ldots \ldots \ldots$

$2.2 .1 \quad$ Optical microscopy $\ldots \ldots \ldots \ldots$ 
2.2 .2 Atomic Force Microscopy $(\mathrm{AFM})$. . . . . . . . . . . . . . 51

2.2 .3 Rheometry . . . . . . . . . . . . . . . 57

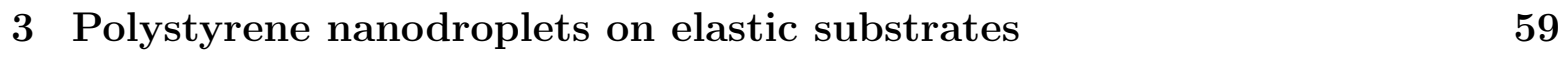

3.1 From rigid to deformable substrates . . . . . . . . . . . . . . . . . . 59

3.2 Imaging the deformed PS/PDMS interface . . . . . . . . . . . . . . . 61

$3.3 \quad$ Extraction of the substrate elastic modulus for small droplets . . . . . . . 63

3.4 Comparison with the Linear Elasticity Theory . . . . . . . . . . . . . . . 64

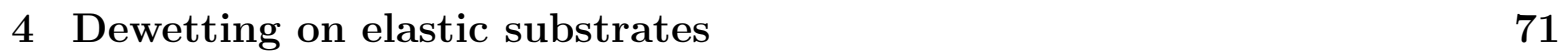

4.1 State of the art - Dewetting on rigid substrates . . . . . . . . . . . 71

4.2 Dewetting dynamics on rubber elastic substrates . . . . . . . . . . . . . . 75

4.2 .1 Dewetting rates . . . . . . . . . . . . . . . . . 75

4.2 .2 Rim profile . . . . . . . . . . . . . . . . 78

4.2 .3 Comparison with a theoretical model . . . . . . . . . . . . . . 83

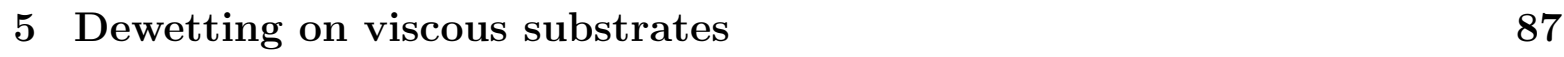

5.1 Dewetting dynamics . . . . . . . . . . . . . . . . . . 89

$5.1 .1 \quad$ Dewetting rates $\ldots \ldots \ldots \ldots$. . . . . . . . . . . . . . . . . 89

5.1 .2 Rim profile . . . . . . . . . . . . . . . 93

5.1 .3 Comparison with numerical simulations . . . . . . . . . . . . . . . 97

5.1 .4 Late stage dewetting . . . . . . . . . . . . . . . . . . 100

6 Topographically structured elastic substrates 103

6.1 Rigid topographically structured substrates . . . . . . . . . . . . . . . . . 104

6.1 .1 Triangular grooves . . . . . . . . . . . . . . . . . . . . 104

6.1 .2 Rectangular grooves . . . . . . . . . . . . . . 107

6.2 Rubber elastic substrates with rectangular grooves . . . . . . . . . . . . . 109

6.2 .1 Results and discussion . . . . . . . . . . . . . . . . 111

$\begin{array}{lll}7 & \text { Summary and outlook } & 119\end{array}$

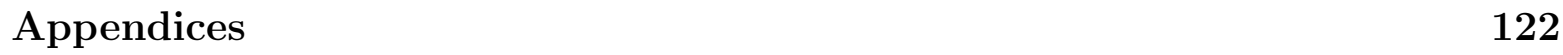

\begin{tabular}{|l|l|}
\hline A Notes on the preparation of the PDMS substrates & 125
\end{tabular}

\begin{tabular}{lr}
\hline B Model - Rubber elastic substrates & 129
\end{tabular} 


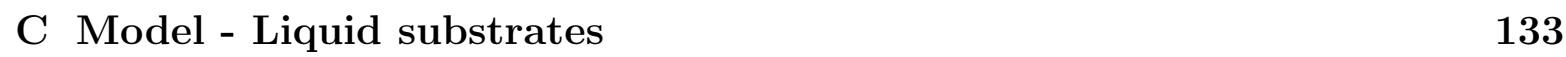

\begin{tabular}{lr}
\hline Bibliography & 137
\end{tabular}

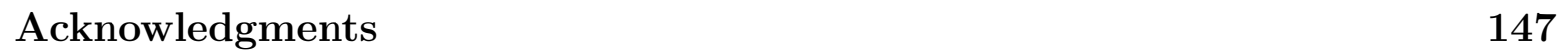





\section{List of Figures}

1.1 Optical images of (a) PS(64k) film dewetting at $150^{\circ} \mathrm{C}$ on an OTS coated Si wafer, (b) PS(100k) film dewetting at $170^{\circ} \mathrm{C}$ on PMMA (c) PS(18k) film dewetting at $120^{\circ} \mathrm{C}$ on PDMS. . . . . . . . . . . . . . . . 5

1.2 Sketch of the temporal evolution of a liquid morphology dewetting/spreading (from left to right/from right to left respectively) on the substrate. The contact radius of the liquid on the solid decreases, respectively increases with time, until the liquid has reached the most favourable configuration that allows the system to minimize its energy. . . . . . . . . . . . . . . . 6

1.3 (a) In the middle of a liquid, a molecule is pulled in all directions resulting in a zero overall force, (b) On the surface of the liquid, the overall force is directed towards the interior. (Image courtesy of David Ansell [1].) . . 7

1.4 Laplace pressure across an air/liquid interface. . . . . . . . . . . . . . . . 8

1.5 Schematic representation of a droplet on a planar surface. The contact angle $\theta_{e}$ is determined by a force balance of the three surface tensions $\gamma_{L V}, \gamma_{S L}$ and $\gamma_{S V}$. (a) Complete wetting for $\theta_{e}=0^{\circ}$, (b) partial wetting for $0^{\circ}<\theta_{e}<180^{\circ}$ and (c) non-wetting for $\theta_{e}=180^{\circ}$. S, L and V denote the solid, liquid and vapour phase. . . . . . . . . . . . . . . . . . 10

1.6 Graph of the gravitational and surface tension force as a function of length scale. The shaded area denotes the area where surface tension forces dominate, whereas in the white area dominate the gravitational forces. . 11

1.7 Effective interface potential $\Phi$ as a function of film thickness $h$. The black curve corresponds to the stable case, the red one to the metastable and the blue curve corresponds to the unstable case. . . . . . . . . . . . . . . 14

1.8 Slippage of a thin liquid film on a solid surface. (a) no-slip $b=0$, (b) full-slip with slip length $b=\infty$ and (c) partial-slip with a finite slip length $b 16$ 
1.9 Profile of a moving front of a hole. (a) AFM image of a part of a dewetting hole. The dashed arrow indicates the direction of the rim motion. (b) A cross section taken in the radial direction of the rim (red solid line in (a)) gives the rim profile (triangles) which is well fitted by an exponentially decaying oscillation (red dashed line). The inset shows the enlarged view of the oscillation. (c) Data of (b) in a 1 : 1 scale. (Image courtesy of R. Fetzer.) .......................... 17

1.10 Optical micrographs showing (a) a polymer droplet on a topographically structured substrate with rectangular grooves, (b) a hexaethylene glycol droplet deposited on a planar hydrophobic substrate bearing horizontal wettable stripes with a periodicity of $400 \mathrm{~nm}$. In both cases, pinning of the three phase contact line is clearly visible from the non-spherical shape of the droplet's perimeter. (Image courtesy of S. Herminghaus.) . . . . . 18

1.11 Sketch of a wetting front moving over (a) a topographic step and (b) a wettability step, and corresponding pinning of the three-phase contact line (patterned area). . . . . . . . . . . . . . . . . . . . . 19

1.12 Temperature dependence of relaxation times in a glass-forming liquid. . . 22

1.13 A shear stress $\sigma$ is applied to the top of the cube, while the bottom is held in place. This stress results in a deformation or strain $\epsilon$, changing the cube into a parallelepiped. . . . . . . . . . . . . . . . . . 24

1.14 Pitch drop flowing through a funnel, University of Queensland in Brisbane, Australia. . . . . . . . . . . . . . . . . . 27

1.15 Schematic strain response of a viscoelastic material to a shear stress applied at time $t=0$ and subsequently held constant. . . . . . . . . . . . . 28

1.16 Some possible responses of a fluid to an applied stress: (a) Newtonian, (b) Shear thinning, (c) Shear thickening. . . . . . . . . . . . . . . . . . . 29

1.17 Stress-strain curve for a typical elastomer. . . . . . . . . . . . . . . . . . 31

1.18 Schematic representation of a Maxwell element. . . . . . . . . . . . . . . 32

1.19 Schematic representation of a Kelvin-Voigt element. . . . . . . . . . . . . 34

1.20 Strain-time relationships at constant stress for simple models: (a) Hookean solid (ideal elastic spring), (b) Newtonian fluid (dashpot) , (c) Maxwell element, (d) Kelvin-Voigt element . . . . . . . . . . . . . . . . . . . . 35

1.21 Schematic representation of a Standard Linear Solid model. . . . . . . . . 36

1.22 Schematic representation of a generalized Maxwell model. . . . . . . . . . 37 
2.1 A typical sample used in our experiments: the lower layer is a piece of Silicon wafer. The intermediate layer is the viscoelastic substrate (rubber or liquid) and the upper layer is the dewetting polymer film. . . . . . . . 40

$2.2 \quad$ Effective interface potential $\Phi$ as a function of film thickness $h$ for the

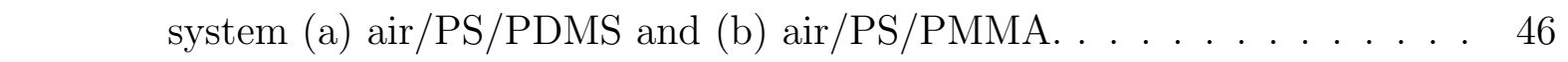

2.3 Diagram illustrating the fabrication of a PDMS stamp. . . . . . . . . 47

2.4 Schematic representation of an Octadecyltrichlorosilane (OTS) molecule. 47

2.5 Diagram illustrating the light path through a dark field microscope. . . . 49

2.6 Schematic representation of a confocal microscope. . . . . . . . . . 50

2.7 General principle of an Atomic Force Microscope. Force acting on the probe tip causes the cantilever to bend, action that is monitored by a sensor. The signal is then used in the scanner feedback, which regulates the sample-tip interaction. . . . . . . . . . . . . . . . . 52

2.8 Force-distance curve describing a single approach-retract cycle of the AFM tip. The AFM tip is approaching the sample surface (1). The initial contact between the tip and the surface is mediated by the attractive van der Waals forces (contact) that lead to an attraction of the tip towards the surface (2). Hence, the tip applies a constant and default force upon the surface that leads to sample indentation and cantilever deflection (3). Subsequently, the tip tries to retract and to break loose from the surface (4). Various adhesive forces between the sample and the AFM tip, however, hamper the tip retraction. These adhesive forces can be taken directly from the force-distance curve (5). The tip withdraws and loses contact to the surface upon overcoming the adhesive forces (6). 53

2.9 Sketch of the forces between the tip and the sample, highlighting where typical imaging modes are operative. . . . . . . . . . . . . . 54

2.10 (a) Front side tip profile, (b) Lateral tip profile. . . . . . . . . . . . . 55

2.11 SEM image of a Harmonix ${ }^{\mathrm{TM}}$ cantilever used to quantitatively map in real-time elastic and dissipative forces during Tapping ${ }^{\mathrm{TM}}$ mode imaging. 56

2.12 Sketch of shear rheometers of different geometries: (a) capillary (b) rota-

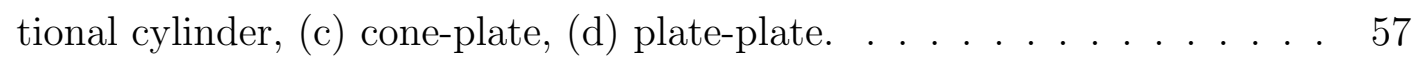


3.1 Sketch of a droplet on a viscoelastic substrate. The dotted line represents the solid without the deformation caused by the vertical component of the liquid surface tension, $\gamma_{L V} \cdot \sin \theta_{e}$. The system has not been sketched to scale, as the wetting ridge near the three-phase contact line of the solid (S)/liquid (L)/vapour (V) phases has been emphasized in order to allow for a better viewing. . . . . . . . . . . . . . . . . 60

3.2 Schematic representation of the lift-off technique. . . . . . . . . . . . . 61

3.3 (a) Cross-section of an AFM scan of the air/PS interface of a sessile droplet of radius $r$ on PDMS (open circles) and the fit to a circular arc (green solid line). (b) Cross-section of an AFM scan corresponding to the PS/PDMS interface of a sessile droplet causing an indentation depth $\delta$ on the substrate (open circles) and the fit to a circle (green solid line). (c) Combination of the cross-sections (a) and (b) showing the complete droplet profile. . . . . . . . . . . . . . . . . . 62

3.4 Theoretical and experimental profile of a deformed PDMS substrate (mixture 1:6 of SG184:SG186) below a sessile PS droplet. The blue dashed curve represents the experimental profile obtained by AFM, whereas the red solid line is the calculated profile according to Rusanov's model (Equation 3.7). . . . . . . . . . . . . . . . . 66

3.5 Theoretical and experimental profile of a deformed PDMS substrate (SG186) at the dry side of the TPCL. The blue curve represents the experimental profile obtained by AFM, whereas the red solid line is the calculated profile according to Rusanov's model (Equation 3.8 ). . . . . . . . . . . . 67

3.6 (a) Top: 3d surface plot of an AFM scan of a PS droplet (blue) on a rubber substrate of PDMS (green) and a highlight of the region of interest (ROI). Bottom: Top view of an AFM scan of the ROI and a cross-section (red line). (b) Graphs of the cross-sections of AFM scans of the height and elasticity signals. (c) Plot of the vertical displacement of the TPCL as a function of substrate elasticity (data points) and the theoretical curve calculated from Rusanov's model (solid line). The values for the elasticities used in this plot are calculated based on Rusanov's model reported in the first column of Table|3.2] . . . . . . . . . . . . . . . . . . . . . . . 69 
4.1 (a)-(b): Sketch of the flow profiles of the two possible mechanisms of energy dissipation and the growth law of the holes. $r$ denotes the hole radius and $t$ the annealing time. (c): Typical behavior of the hole radius as a function of time. Blue dashed curve: the theoretical prediction for the case where energy dissipation is dominated by viscous flow. Green dotted curve: the theoretical prediction for the case where dissipation takes place only by friction at the interface. Red solid curve: theory [2] fitted to the experimental data for the real case where both viscous flow and friction at the interface are present. (Image courtesy of K. Jacobs.) . 73

4.2 (a) Rim profiles of a $130 \mathrm{~nm}$ PS film on OTS and DTS covered Si wafers. The inset depicts a semilog plot of $|h(x)-H|$, where $H$ is the PS film \begin{aligned} \hline thickness and (b) the corresponding dewetting rates on the two different \\ \hline\end{aligned} substrates. (Images courtesy of R. Fetzer) . . . . . . . . . . . . . . . . . 74

4.3 (a) Time series of a PS(17.4k) film with a film thickness of $150 \mathrm{~nm}$ dewet-

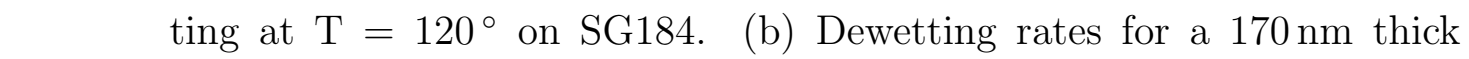
PS(17.4k) film at $120^{\circ} \mathrm{C}$ dewetting on substrates of different elasticities. In the tabular are given the ratios with which SG184 and SG186 are mixed in order to produce substrates with intermediate elasticities. . . . . . . . 76

4.4 Normalized dewetting velocities of a PS(17.4k) film with thickness of $150 \mathrm{~nm}$ dewetting at $\mathrm{T}=120^{\circ}$ on substrates of different elasticities as a function of the substrate elasticity (data points) and the exponential fit to the experimental results (solid curve). . . . . . . . . . . . . . . 77

4.5 (a) AFM scan of the liquid/air interface of a typical hole of PS dewetting on PDMS, (b) AFM scan corresponding to the liquid/rubber interface around the hole, (c) Radial cross section of the hole and the corresponding substrate deformation. . . . . . . . . . . . . . . . . . . . 79

4.6 Cross-sections of AFM scans of rim profiles at increasing annealing time. The hole radii are $9 \mu m, 12 \mu m, 15 \mu m$ and $18 \mu m$ in $150 \mathrm{~nm}$ thick PS(17.4k) films dewetting on SG186 at $120^{\circ} \mathrm{C}$. . . . . . . . . . . . . . . . . . . . . 80

4.7 (a) Rim height $h$ and Full Width at Half Maximum $F W H M$ as a function of the normalized hole radius (b) depth $d_{t}$ of the undulation as a function of hole radius, (c) width $W$ of the upper part of the rim and width $W_{i}$ of the substrate deformation as a function of hole radius and (d) indentation depth $d_{i}$ of the deformed substrate as a function of hole radius. . . . . . . 81 
4.8 (a) Rim profiles of PS(17.4k) dewetting at $120^{\circ} \mathrm{C}$ on substrates of different elasticities. The film thickness and hole radius are kept constant in both systems. (b) Two of the rim profiles of Graph.(a) on SG186 (softest substrate) and SG184 (stiffest substrate). . . . . . . . . . . . . . . 82

4.9 Cross section of an AMF scan of the liquid/rubber interface below a rim of PS(17.4k) dewetting on SG186 at $120^{\circ} \mathrm{C}$ (green dotted curve) compared to the deformation calculated taking into consideration only the Laplace pressure inside the liquid (red solid curve). The radius of the hole is $r=9 \mu m . \ldots \ldots \ldots$. . . . . . . . . . . . . . . . . 84

4.10 Cross section of an AMF scan of the liquid/rubber interface below a rim of PS(17.4k) dewetting on SG186 at $120^{\circ} \mathrm{C}$ (green dotted curve) compared to the deformation calculated taking into consideration only the Laplace pressure inside the liquid (red solid curve). The radius of the hole is $r=12 \mu m$. . . . . . . . . . . . . . . . . . . . 85

$5.1 \quad$ Schematic diagram of the von Neumann construction showing the rim of the dewetting film. . . . . . . . . . . . . . . . . . . . . . . . . 88

5.2 Dewetting of a liquid film A on a liquid substrate B on top of a silicon substrate. By $h_{A}$ and $h_{B}$ we denote the (relative) positions of the interfaces above the substrate surface. . . . . . . . . . . . . . . . . . . . . . . . . . 90

5.3 Dewetting rates of (a) a PS(100k) film and (b) a PS(17.4k) film dewetting at $160{ }^{\circ} \mathrm{C}$ on PMMA substrates of different molecular weights. . . . . . . 91

5.4 Dewetting velocities as a function of substrate molecular weight for $50 \mathrm{~nm}$ thick PS films dewetting on PMMA at $160^{\circ} \mathrm{C}$. The solid lines are guides to the eye. . . . . . . . . . . . . . . . . . . . . . 92

5.5 Cross-sections of AFM scans of the rim and underlying substrate deformation of a PS(17.4k) film dewetting at $160^{\circ} \mathrm{C}$ on a (a) PMMA(62k) substrate and (b) PMMA(520k) substrate. . . . . . . . . . . . . . . . . . 94

5.6 (a) Cross-section of an AFM scan of the rim and underlying substrate deformation of a $50 \mathrm{~nm}$ thick PS(17.4k) film dewetting at $160^{\circ} \mathrm{C}$ on PMMA(62k).

(b) Cross-section of an AFM scan of the rim and underlying substrate deformation of a $100 \mathrm{~nm}$ thick $\mathrm{PS}(100 \mathrm{k})$ film dewetting at $160^{\circ} \mathrm{C}$ on

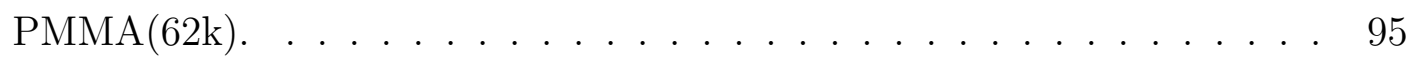


5.7 Cross-sections of AFM scans of the rim and underlying substrate deformation of a growing hole of a PS(17.4k) film dewetting at $170{ }^{\circ} \mathrm{C}$ on PMMA(10k) with (a) $r_{1}=4 \mu m$ and (b) $r_{2}=17 \mu m$. . . . . . . . . . . 96

5.8 Cross-sections of AFM scans of the rim and underlying substrate deformation of a $4 \mu \mathrm{m}$ hole of a $\mathrm{PS}(17.4 \mathrm{k})$ film dewetting at $170{ }^{\circ} \mathrm{C}$ on a (a) PMMA(4k) substrate and (b) PMMA(10k) substrate. . . . . . . . . . . . 97

5.9 (a) Experimental results and (b) simulations for the dewetting rates of a $50 \mathrm{~nm}$ PS (17.4k) film dewetting at $170^{\circ} \mathrm{C}$ on PMMA substrates of different molecular weights. . . . . . . . . . . . . . . . 98

5.10 (a) Experimental results of the rim and interface deformation of a growing hole $\left(r_{1}=4 \mu m\right.$ and $\left.r_{2}=10 \mu m\right)$ of a PS $(17.4 \mathrm{k})$ film dewetting at $170^{\circ} \mathrm{C}$ \begin{tabular}{|c|c|c|c|}
\hline \hline on PMMA (4k). (b) Solutions of Equation & C.6 & of the rim and interface \\
\hline
\end{tabular} deformation of a PS (17.4k) film dewetting at $170^{\circ} \mathrm{C}$ on a PMMA (4k) substrate for the same hole radii. The simulation parameters are $\alpha=2.3$, $\gamma=0.2, h *=0.02 H, \phi *=4.33, H=200 \mathrm{~nm}$ and initial data are $h_{1}=H$ and $h_{2}$ is a smooth step function with values $H+h *$ for $R<0.3 \mu m$ and $H+35 \mathrm{~nm}$ for $R>0.5 \mu \mathrm{m}$. . . . . . . . . . . . . . . . . . . . . . . 99

\begin{tabular}{|l|l|l|l}
5.11 & Solutions of Equation & C.6 & of the rim and interface deformation of a PS
\end{tabular} (17.4k) film dewetting at $170^{\circ} \mathrm{C}$ on a PMMA (4k) substrate for the same hole radii. The varied simulation parameters are: (a) $\alpha=23, \gamma=0.4$, (b) $\alpha=0.23, \gamma=0.4$ and (c) $\alpha=0.23, \gamma=4.0$. . . . . . . . . . . . . . 100

5.12 3d AFM image of a PS(17.4k) droplet on PMMA(4k) after 24 hours of annealing at $170^{\circ} \mathrm{C}$. The droplet has not reached equilibrium yet. . . . . 101

5.13 Time series of droplets of PS(17.4k) on PMMA(4k) obtained from numerical simulations. . . . . . . . . . . . . . . . . . 101

6.1 Sketch of infinite wedges with wedge angle $\psi(\mathrm{a})$, including a wetting morphology for small liquid volume and low contact angle $\theta<\psi(b)$, including a wetting morphology with high contact angle $\theta>\psi(\mathrm{c})$, and corresponding AFM images (d, e, f). (Image courtesy of K. Khare.) . . . 104

6.2 Wetting morphologies in a triangular groove. (a), (b) and (c) show sketches of morphologies for increasing contact angle. Corresponding AFM micrographs are shown in (d), (e) and (f). (Image courtesy of K. Khare.) . . . . . . . . . . . . . . . . . . 105 
6.3 $\quad$ Morphology diagram for a liquid with contact angle $\theta<90^{\circ}$ in a triangular groove of wedge angle $\psi$. . . . . . . . . . . . . . . . . . 106

6.4 AFM images of liquid morphologies in rectangular grooves. For $\theta<$ $45^{\circ}$ (top row) one can observe (a) droplets connected to a pinned wedge [D/pW], (b) extended filaments with positive Laplace pressure connected to a pinned wedge $\left[F^{+} / \mathrm{pW}\right]$ and $(\mathrm{c})$ extended filaments with negative Laplace pressure connected to a pinned wedge $\left[F^{-} / \mathrm{pW}\right]$. For $\theta>45^{\circ}$ (bottom row), one can observe the same basic morphologies without the thin liquid wedges [W] in the groove corners, i.e. (d) overspilling droplets [D] that spread on the ridges, (e) extended filaments with positive Laplace pressure $\left[F^{+}\right]$and (f) extended filaments with negative Laplace pressure $\left[F^{-}\right]$. (Image courtesy of R. Seemann.) . . . . . . . . . . . . . . 107

6.5 Morphology diagram of a rectangular groove as a function of groove aspect ratio $X$ and liquid contact angle $\theta<90^{\circ}$. (Image courtesy of R. Seemann.) 108

6.6 Sketch of a cross section of a topographically structured substrate with rectangular grooves. The parameters used for the characterisation of the sample are the groove width $G$, the ridge width $R$ and its height $H$. . . . 110

6.7 3D projection of a confocal scanning laser microscopy image of a topographically structured Si master with rectangular grooves. . . . . . . . . 110

6.8 Optical micrograph of monodisperse water droplets in rectangular grooves of a topographically structured PDMS substrate. . . . . . . . . . . . . . 111

6.9 Close-up of an optical micrograph of nearby droplets deforming the ridges of the groove in which they are contained. . . . . . . . . . . . . . . . . . 112

6.10 Optical micrograph at the late stage of water condensation on a topographically structured PDMS substrate with high ridge aspect ratio. Filaments can be observed to be arranged in a laterally periodic pattern. Highlighted in the red circle is a filament overspilling on the ridges. . . . 113

6.11 Optical micrograph of the liquid morphologies on a PDMS substrate with rectangular grooves of large ridge aspect ratio and low groove width. . . . 114

6.12 Optical micrograph showing liquid morphologies on a PDMS substrate with rectangular grooves of low ridge aspect ratio and large groove width. 115 
6.13 (a) Histogram of the normalized filament length for the two cases shown in Figure 6.11 and Figure 6.12 , The distribution for the sample with the lower aspect ratio and higher groove width (Figure 6.12 ) is wider and presents a higher mean value than that for the sample with the higher aspect ratio and the smaller groove width (Figure 6.11). (b) Contour plot of the filament length as a function of ridge aspect ratio and groove width. The highest value of filament length can be achieved by increasing the groove width and decreasing the ridge aspect ratio, while, towards lower groove widths and higher aspect ratios, the preferred filament length becomes smaller. . . . . . . . . . . . . . . . . . . 116

6.14 Optical micrograph of a hydrophilized PDMS substrate after water condensation has taken place. The ridges collapse along the length of the groove similar to a closing zipper. . . . . . . . . . . . . . . . . . . . . . 117

6.15 Schematic rapresentation of the "zipper effect". . . . . . . . . . . . . . . 118

A.1 Dewetting rates of a $150 \mathrm{~nm}$ thick PS(17.4k) film on SG184 substrates of different aging. . . . . . . . . . . . . . . . 125

A.2 Dewetting rates for a $150 \mathrm{~nm}$ thick PS(17.4k) film dewetting on SG184 at $120^{\circ} \mathrm{C} \ldots \ldots \ldots \ldots \ldots \ldots$

A.3 Dewetting rates for a $150 \mathrm{~nm}$ thick PS(17.4k) film dewetting on SG184 at $120^{\circ} \mathrm{C} \ldots \ldots \ldots \ldots \ldots \ldots$

A.4 Dewetting rates for a $150 \mathrm{~nm}$ thick PS(17.4k) film dewetting on SG186 at $120^{\circ} \mathrm{C} \ldots \ldots \ldots \ldots 128$ 



\section{List of Symbols}

$\gamma$ Surface tension . . . . . . . . . . . . . . . . . . . . 7

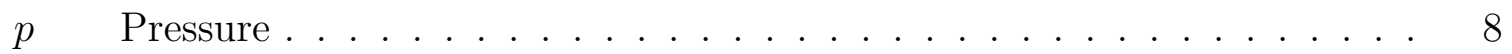

$\Delta P \quad$ Laplcare pressure . . . . . . . . . . . . . . . . . . . . . . . . . . 8

c $\quad$ Mean curvature . . . . . . . . . . . . . . . . . . . . . . . 8

$\theta_{e} \quad$ Equilibrium contact angle . . . . . . . . . . . . . . . . . . . . . . 9

$\rho$ Density . . . . . . . . . . . . . . . . . . . . . . . 10

g $\quad$ Gravitational acceleration . . . . . . . . . . . . . . . . . . . 10

$l_{c} \quad$ Capillary length . . . . . . . . . . . . . . . . . . . . . . . 11

S Spreading coefficient . . . . . . . . . . . . . . . . 12

W Energy per unit area . . . . . . . . . . . . . . . . . . . . 13

A Hamaker constant . . . . . . . . . . . . . . . . . 13

$\Phi \quad$ Effective interface potential . . . . . . . . . . . . . . . . . . 13

$\lambda_{s}$ Spinodal wavelength . . . . . . . . . . . . . . . . . . . 14

b $\quad$ Slip length $\ldots \ldots \ldots \ldots$. . . . . . . . . . . . . . . . . . 16

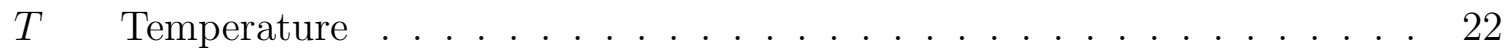

$T_{g} \quad$ Glass transition temperature . . . . . . . . . . . . . . . . 22

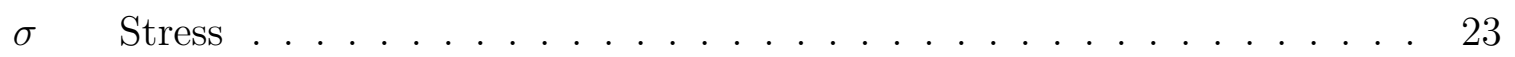

Frorce . . . . . . . . . . . . . . . . . . . . . . . 23

€ Strain . . . . . . . . . . . . . . . . . . . . . 24

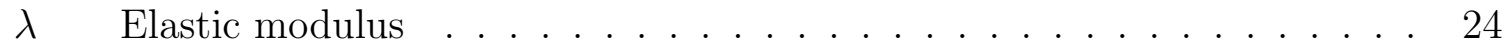

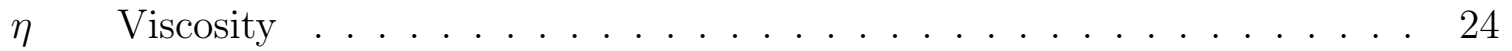

E $\quad$ Young's modulus . . . . . . . . . . . . . . . . . . . 25

G Shear modulus . . . . . . . . . . . . . . . . . 25

K $\quad$ Bulk modulus . . . . . . . . . . . . . . . . . . . . . 26

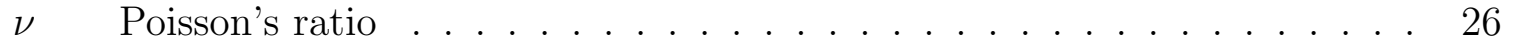

Trelaxation time . . . . . . . . . . . . . . . . . . 27 


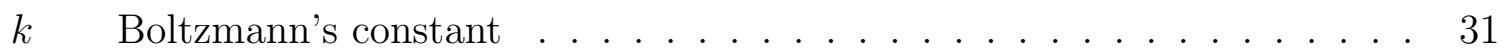

$M_{n}$ Molecular number $\ldots \ldots \ldots \ldots \ldots \ldots$

$M_{w} \quad$ Molecular weight $\ldots \ldots \ldots \ldots \ldots \ldots$. . . . . . . . . . . . . 44

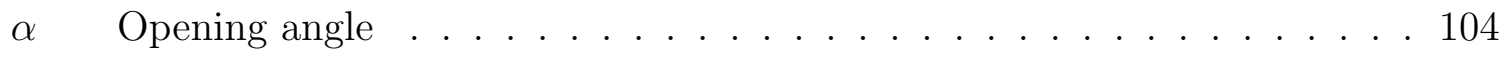

$\psi$

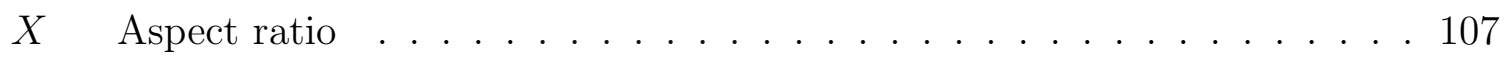

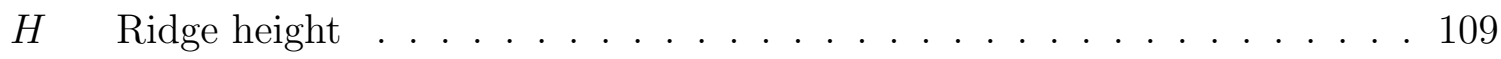

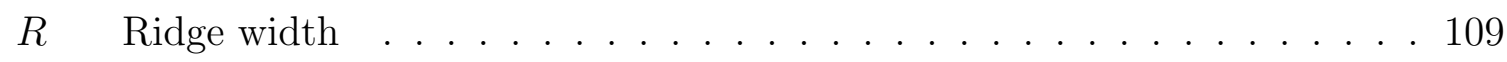




\section{Introduction}

In recent years substantial efforts have been invested in miniaturizing chemical processes by building microfluidic systems. The Lab-on-a-chip (LOC) concept integrates a great variety of chemical and physical processes into a single device in a similar way that an integrated circuit incorporates many electronic devices into a single chip [3]. These microfluidic devices not only do they allow for cheap mass production, but they can operate with much smaller quantities of reactants and reaction products than standard laboratory equipment. This is particularly important for rare and expensive substances such as some biological material and for toxic or explosive elements [4].

One of the most promising areas of microfluidics is its potential for integration of nanofluidic systems into it, e.g. in Micro Total Analysis System ( $\mu$-TAS) technology, in which scientists are trying to embody and miniaturize desktop laboratory procedures on hand-held micro and nanofluidic chips. For instance, NCAMs (Neural Cell Adhesion Molecules), when incorporated into microfluidic devices, can reproducibly perform digital switching, allowing transfer of fluid from one microfluidic channel to another [5], selectively separate and transfer analytes by size and mass [5] 9], mix reactants efficiently [10] and separate fluids with disparate characteristics [5, 11]. These technologies have already found application in various fields such as biotechnology, chemistry and engineering.

Besides meeting technical challenges, new theoretical concepts are needed to understand the basic physical processes underlying this new technology [12, 13]. Similarly to the ultimate limits for the miniaturization of electronic devices being set by quantum fluctuations, in a chemical chip these limits are determined by thermal fluctuations and can be explored by methods of classical statistical mechanics. When structures approach the molecular size regime, new physical constraints are placed on the behaviour of the 
fluid that induce it to exhibit new properties not observed in bulk. Some examples of such small scale effects are:

- surface energy effects dominate over gravitational effects,

- slip boundary conditions might come into play,

- the flow's Reynolds number becomes small, which can create problems e.g. in small scale mixing devices.

The need for such smaller and efficient fluidic systems has resulted in a variety of nanofabrication techniques to produce micro and nanochannels in silicon, glass and polymers. Each fabrication method is capable of producing micro or nanoscale channels, however some methods are time-consuming while others are costly or require expensive machines/equipments, which makes them unsuitable for small-scale production.

Much of the exploratory research in microfluidic systems has been carried out in Polydimethylsiloxane (PDMS), a polymer, the properties of which are entirely distinct from those of silicon [14, 15] or glass. Among the advantages that have made it a key material for exploratory research and research engineering are its optical transparency, low toxicity, inexpensive and uncomplicated clean-room fabrication and its ability to support certain very useful components such as pneumatic valves [16].

In order to better exploit the potentialities that a microfluidic system made of PDMS can offer, we need to gain knowledge of how various liquids behave on such a substrate. Based on the good understanding of the spreading dynamics and morphology of liquid thin films on rigid substrates, the aim of this thesis is to address the more complicated problem of dewetting on viscoelastic substrates. Specifically, we are interested in exploring how the viscoelastic properties of the substrate influence the dewetting dynamics and statics of the overlying polymer thin film and which are the mechanisms that dominate the dewetting process.

This thesis is divided into seven chapters.

The first chapter is dedicated to the theoretical background required for the good understanding of the following chapters. We give an overview of the main theoretical aspects and the state-of-the-art of wetting and dewetting. In this study, polymers are employed not only as the dewetting liquid, but also as viscoelastic substrates onto which dewetting takes place. Therefore, in the second part of this chapter we discuss the 
properties of polymers and what makes them such valuable tools in exploring dewetting phenomena.

In the second chapter, we firstly describe the preparation methods to fabricate the systems used in our experiments. Following, we present the characterization techniques applied in our experimental work and we give a short introduction to the physical principles of operation of each technique.

The shape of sessile liquid droplets on rubber elastic substrates and the deformation they cause on the substrate is discussed in the third chapter.

In the fourth chapter we tune the elasticity of the substrate and examine how the dynamics of dewetting are directly connected to the elastic properties of the underlying substrate.

In chapter five we vary the viscosity of the substrate and observe how this affects the dewetting process. Additionally, we propose a theoretical model that describes our system and has been developed in collaboration with B. Wagner and A. Münch. The outcome of the simulations carried out based on this model are presented and confronted with the experimental results .

In chapter six, some preliminary experiments on wetting phenomena on topographically structured viscoelastic substrates will be introduced and discussed.

Finally, in the last chapter, we summarize the main contributions of the thesis and point out some open problems and future research perspectives. 



\section{Chapter 1}

\section{Theoretical Background}

\subsection{Fundamentals of wetting and dewetting}

Varnish manufacturers advise to clean the surface to be painted. What will happen if we paint a dusty or oily substrate? The varnish film will bead up into droplets and dewet on the substrate before it has time to cure into a homogeneous film. Similarly, if we pour oil in a Teflon $\AA$ coated frying pan, unless the oil film thickness is above a certain length in order to be stabilized by gravity, the oil will not spread on the pan. From the above examples, it is evident that stability conditions play an important role in dewetting phenomena. When conditions exist such that the film acquires sufficient mobility, destabilization will occur through the formation of dewetting patterns at the free interface, which grow over time.

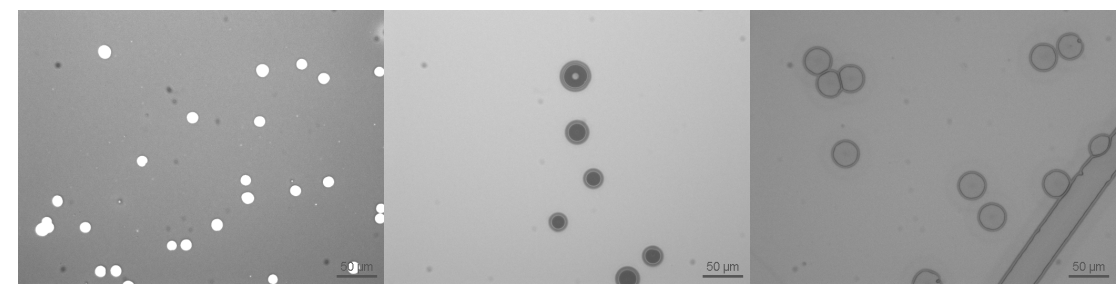

(a)

(b)

(c)

Figure 1.1: Optical images of (a) $\mathrm{PS}(64 \mathrm{k})$ film dewetting at $150^{\circ} \mathrm{C}$ on an OTS coated Si wafer, (b) PS(100k) film dewetting at $170^{\circ} \mathrm{C}$ on PMMA (c) PS(18k) film dewetting at $120^{\circ} \mathrm{C}$ on PDMS. 
Figure 1.1 displays details of the typical rupture scenario of liquid thin polystyrene films dewetting on three different substrates: an OTS coated Si wafer, a liquid PMMA substrate and a cross-linked PDMS substrate. As we can observe, the different combinations of dewetting film and underlying substrate can give rise to a variety of dewetting patterns. Generalizing though, we can affirm that the initially flat film ruptures spontaneously or under the influence of defects, resulting in the formation of tiny dry patches that expose the substrate to the air. The dry patches grow laterally with time and coalesce, resulting in polymer ridges, which then decay into drops. Thus, dewetting (Figure 1.2. from left to right) tends to minimize the film/substrate interface, in contrast to wetting (or spreading) (Figure 1.2 , from right to left), which tends to maximize it.

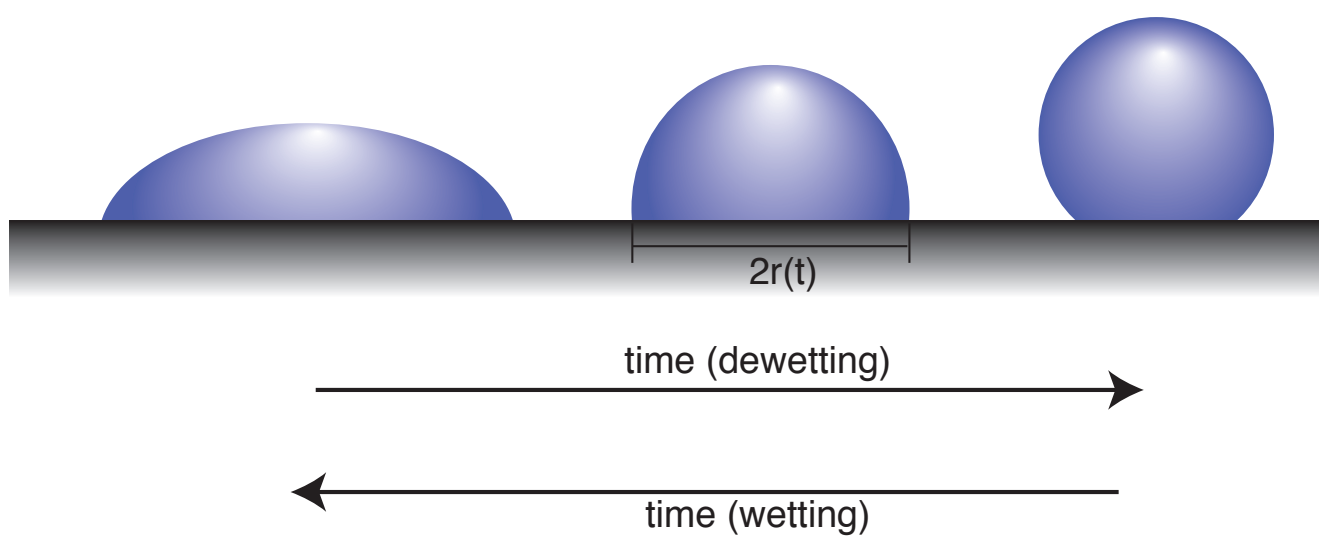

Figure 1.2: Sketch of the temporal evolution of a liquid morphology dewetting/spreading (from left to right/from right to left respectively) on the substrate. The contact radius of the liquid on the solid decreases, respectively increases with time, until the liquid has reached the most favourable configuration that allows the system to minimize its energy.

In the first part of this chapter, the main notions of the theory required for the further reading of this thesis will be discussed. Since this thesis deals with the dewetting on deformable substrates, we will first introduce and examine the behaviour of a liquid in contact with a rigid surface in order to eventually be able to apply these concepts to the deformable substrates. 


\subsubsection{Capillarity}

\section{Surface tension - Free liquid interface}
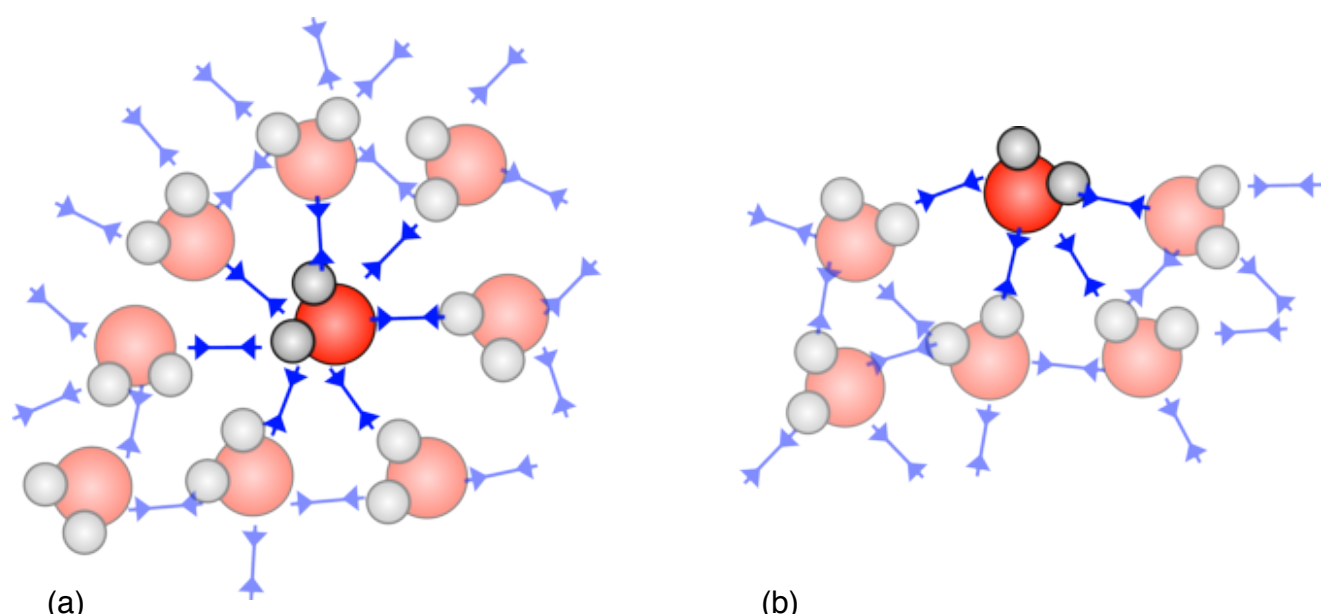

(b)

Figure 1.3: (a) In the middle of a liquid, a molecule is pulled in all directions resulting in a zero overall force, (b) On the surface of the liquid, the overall force is directed towards the interior. (Image courtesy of David Ansell [1].)

A molecule in contact with a neighbouring molecule is in a lower state of energy than if it hadn't been in contact with a neighbour. In the bulk of a liquid, the interior molecules all have as many neighbours as they can possibly get (Figure 1.3.(a)). The boundary molecules though, on the surface of the liquid, have fewer neighbours than the interior molecules, resulting therefore in a higher state of energy (Figure 1.3.(b)).

For the liquid to minimize its energy state, it must minimize its number of boundary molecules and must therefore minimize its surface area. This phenomenon is called surface tension $\gamma$ and it is referred to as a free energy per unit area, although it can be equally thought of as force per unit length.

\section{Laplace pressure}

The curvature of a surface, which locally increases its surface area gives rise to a restoring pressure difference across the liquid/vapour interface. Let us consider a small 


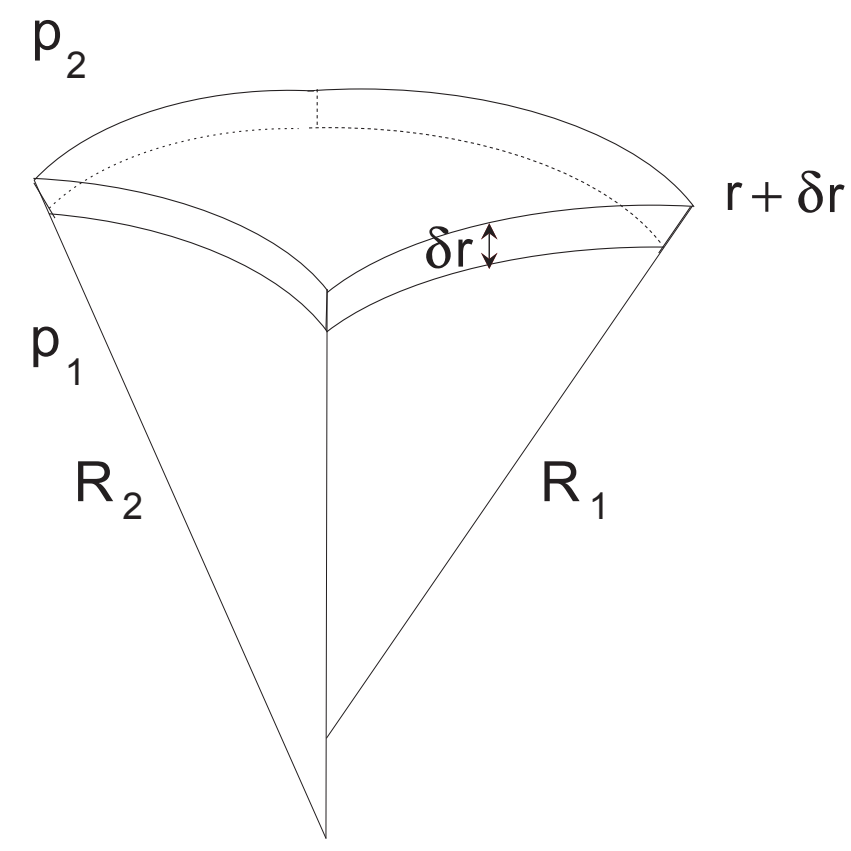

Figure 1.4: Laplace pressure across an air/liquid interface.

displacement $\delta r$ of the interface illustrated in Figure 1.4. For a sufficiently small area, a curved surface can be described in terms of two principal radii of curvature in mutually orthogonal planes, $R_{2}$ and $R_{2}$. The pressures in the liquid and air phase are $p_{1}$ and $p_{2}$ respectively. In equilibrium, the work done to displace the surface parallel to itself over a distance $\delta r$, has to be equal to the work necessary in order to change the surface area of the interface by $\delta A$. The increase in surface area involves an increase in the surface energy $\gamma \delta A$, where $\gamma$ is the liquid/air surface tension. The pressure on the concave side is greater than on the convex side of the liquid interface by an amount depending on the surface tension and on the curvature of the interface. This difference is called Laplace pressure and is defined as:

$$
\Delta P=\left(p_{1}-p_{2}\right)=2 \kappa \gamma
$$

where $c$ is the mean curvature of the curved interface. For any arbitrary surface, the local mean curvature $c=\left(c_{\|}+c_{\perp}\right) / 2$ is the arithmetic mean of the two principal curvatures $c_{\|}$and $c_{\perp}$ in two perpendicular directions at the respective point $x$ of the surface. 


\section{Contact angle and Young's equation - Liquid on a rigid substrate}

Let us consider a liquid drop on a rigid surface. The junction of the two interfaces in contact, forms a curve known as the contact line and the angle that these two interfaces form with each other is called the equilibrium contact angle $\theta_{e}$. If the liquid is very strongly attracted to the rigid surface (for example water on a strongly hydrophilic solid), then the surface will be completely covered by the liquid (wetting), whereas, on highly hydrophobic surfaces, the water forms droplets that simply rest on the surface (nonwetting). The expressions wetting and non-wetting, as employed in various practical situations, tend to be defined in terms of the effect desired. Usually, however, wetting means that the contact angle between a liquid and a solid is zero or so close to zero that the liquid spreads over the solid easily and non-wetting means that the angle is $180^{\circ}$ or close to $180^{\circ}$ such that the liquid tends to ball up and run off the surface easily. The wetting or non-wetting of a liquid that comes into contact with a rigid surface does not depend only on the interfacial properties of the liquid/vapour interface, but also on the interfacial properties of the liquid/solid and solid/vapour interfaces resulting in a certain contact angle of the liquid on the surface.

Summarizing, three possible cases that are shown schematically in Figure 1.5 are observed:

- complete wetting contact angle $\theta_{e}=0^{\circ}$, the liquid wets completely the solid surface (Figure 1.5. (a)),

- non-wetting contact angle $\theta_{e}=180^{\circ}$, the liquid remains as a spherical drop on the solid surface (Figure 1.5.(b)) and

- partial wetting contact angle $0^{\circ}<\theta_{e}<180^{\circ}$, the liquid wets the solid surface only partially and there is a finite liquid/solid surface (Figure 1.5.(c)).

The equilibrium at the three-phase contact line of a solid $(\mathrm{S})$, liquid $(\mathrm{L})$ and vapor $(\mathrm{V})$ is described by the well-known Young's equation [17] in the form:

$$
\gamma_{S V}-\gamma_{S L}=\gamma_{L V} \cos \theta_{e}
$$

where $\gamma_{L V}, \gamma_{S L}$ and $\gamma_{S V}$ represent interfacial tensions, respectively, for the solid/vapour, solid/liquid and liquid/vapour interfaces and $\theta_{e}$ is the equilibrium contact angle. One 


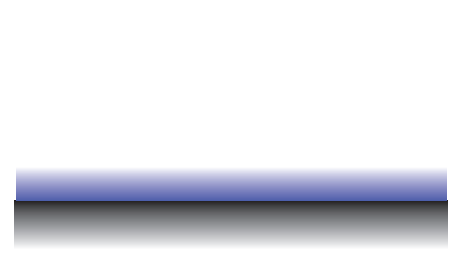

(a)

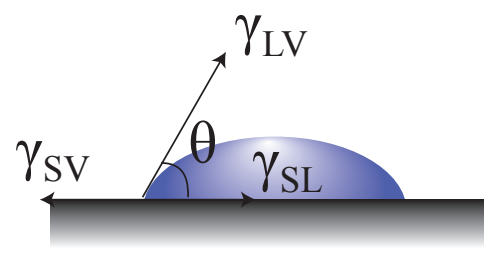

(b)

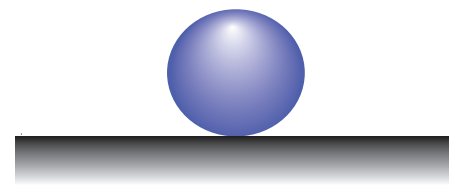

(c)

Figure 1.5: Schematic representation of a droplet on a planar surface. The contact angle $\theta_{e}$ is determined by a force balance of the three surface tensions $\gamma_{L V}, \gamma_{S L}$ and $\gamma_{S V}$. (a) Complete wetting for $\theta_{e}=0^{\circ}$, (b) partial wetting for $0^{\circ}<\theta_{e}<180^{\circ}$ and (c) non-wetting for $\theta_{e}=180^{\circ}$. S, L and $\mathrm{V}$ denote the solid, liquid and vapour phase.

simple way to derive Young's equation is to describe the interfacial tensions as forces per unit length and to consider the local force balance along the $\mathrm{x}$-axis parallel to the solid surface (Figure 1.5.(b)).

\section{Capillary length}

Let's now consider a microscopic liquid droplet hanging from a ceiling. If the droplet is small enough such that the surface tension force overcomes gravity, then the droplet will remain on the ceiling. As we increase its liquid volume, at the crossover point where gravity becomes larger than the force due to surface tension, the droplet will fall off the ceiling under its own weight. As indicated in Figure 1.6, the crossover occurs around the millimetre range. Well below this crossover regime, the force due to surface tension is dominant, whereas well above it, the force due to gravity dominates.

The force due to the surface tension (blue dashed line in Figure 1.6) is:

$$
F_{\gamma}=2 \pi r \gamma
$$

$r$ being the radius of the contact area between the liquid and the wall, while the force due to gravity (green dotted line) for a spherical drop is:

$$
F_{g}=\frac{4}{3} \pi r^{3} \rho g
$$

where $\rho$ is the density of the fluid and $g$ is the acceleration due to gravity. The two forces 


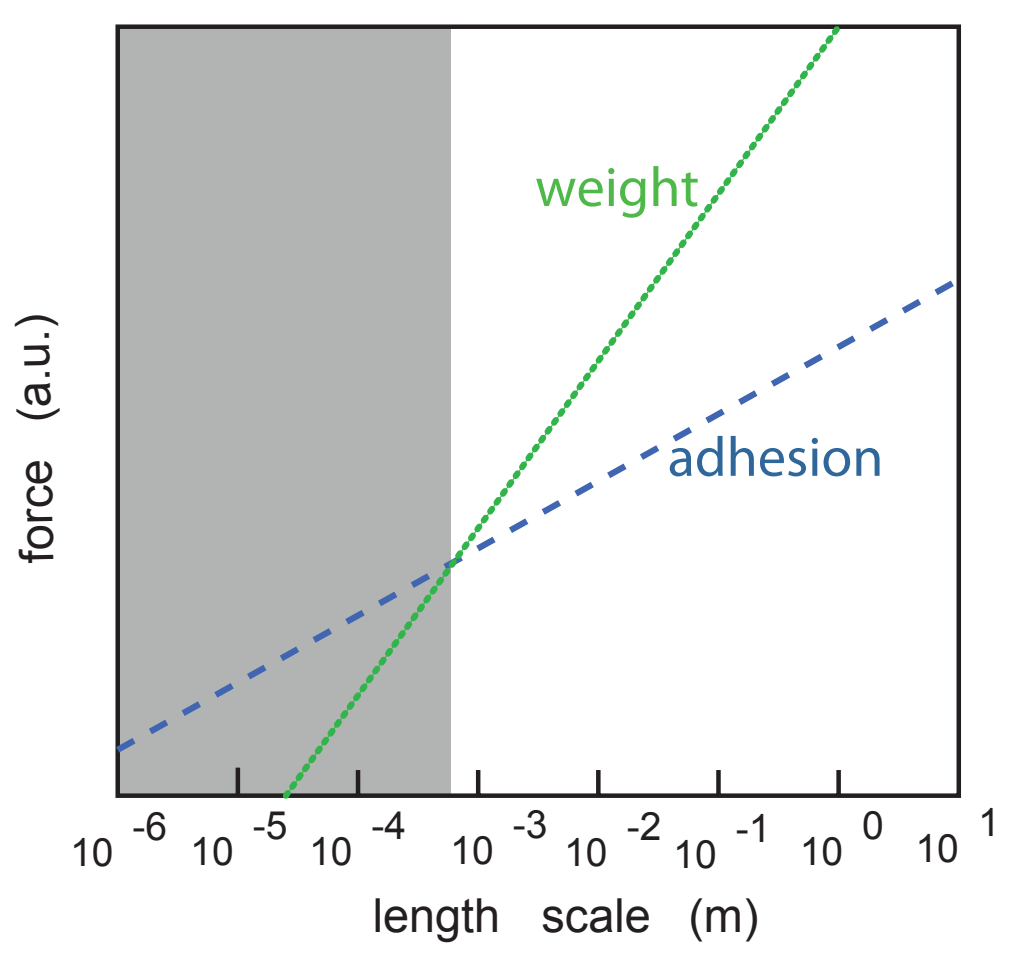

Figure 1.6: Graph of the gravitational and surface tension force as a function of length scale. The shaded area denotes the area where surface tension forces dominate, whereas in the white area dominate the gravitational forces.

are equal when the drop separates from the ceiling. The critical radius of the drop when it separates, is obtained by balancing Equation 1.3 and Equation 1.4

$$
r_{c}=l_{c}=\sqrt{\frac{3 \gamma}{2 \rho g}}
$$

The quantity $l_{c}$ is called capillary length and it defines the length scale below which the surface tension force is dominant and gravity can be considered negligible. As deducted from Equation 1.5, the capillary length varies with the liquid and with the surrounding medium. For example, the capillary length for water at standard temperature and pressure is $3.2 \mathrm{~mm}$, whereas for polystyrene it is $2.1 \mathrm{~mm}$.

In order to avoid gravitational effects, which would make our system more complicated and would not allow for simplifications such as the approximation of a sessile drop to a spherical shape (which is the solution to the Young-Laplace equation for zero gravity), we choose the dimensions of our system to be on a length scale that is significantly 
below the capillary length of polystyrene.

\section{Kinetics-Spreading coefficient}

What happens inbetween the moment a liquid is placed on a surface and the moment the system reaches equilibrium? When a drop of a liquid is put on top of a solid surface, there are two competing effects. The interactions with the solid substrate make it energetically favourable for the drop to spread such that it wets the surface. However, spreading increases the area of contact between the liquid and vapour, which also increases the surface energy between the drop and the vapor. The factor determining if the drop will spread or dewet is the so-called spreading coefficient $S$, which arises from the unbalanced surface forces:

$$
S=\gamma_{S V}-\gamma_{S L}-\gamma_{L V}=\gamma_{L V}(\cos \theta-1)
$$

When $S$ is positive, a thermodynamic wetting angle cannot be achieved and the liquid will spread over the solid surface, whereas a negative spreading coefficient refers to the partial wetting regime with a finite contact angle [18].

\subsubsection{Stability of homogeneous liquid films}

\section{Effective interface potential}

The stability of a thin liquid film on a solid surface is determined by the balance of short and long range interactions between the liquid and the solid. Short range forces arise due to the repulsion of overlapping electron orbitals and vary as a function of $r^{-12}, r$ being the distance between the molecules. Considering two planar surfaces, this

repulsion yields an interaction energy per area varying as $h^{-8}, h$ being the distance between the two surfaces. The long range interactions are caused by correlations in the fluctuating polarizations of nearby molecules and vary as a function of $h^{-6}$. For large distances $(>100 \mathrm{~nm}$ ) retardation effects have to be considered and the interaction falls as $h^{-7}$. 
Considering two planar surfaces, the non-retarded interaction yields [19, 20]:

$$
W(h)=-\frac{A}{12 \pi h^{2}}
$$

where $W$ is the energy per unit area and $A$ is known as the Hamaker constant [21] and is equal to :

$$
A=\pi^{2} \rho_{1} \rho_{2} C
$$

$\rho_{1}$ and $\rho_{2}$ being the molecular number densities of the two surfaces involved. $C$ is a coefficient in the particle-particle interaction and it depends on molecular parameters such as polarizability, but, not on the geometry of the bodies. The definition of the Hamaker constant in Equation 1.8 ignores the influence that neighboring atoms can have on the interaction between any pair of molecules. Even though this approximation is definitely not exact for condensed phase, it turns out that the expression of Equation 1.7 remains valid even within the framework of continuum theories. The only change is in the way the Hamaker constant is calculated [19, 22], namely by the optical properties of the materials involved. For $A<0$, the system can gain energy by increasing the distance $h$ between the surfaces, while for $A>0$, the system gains energy by reducing the distance $h$.

The effective interface potential $\Phi$ is the result of the combination of the short and long range interactions and is defined as the excess free energy per unit area which is necessary to bring two interfaces from infinity to a certain distance and can be characterized by the following expression [23, 24]

$$
\Phi(h)=C^{*} \frac{1}{h^{8}}+W(h)
$$

where $C^{*}$ is a constant characterizing the interaction strength.

\section{Symmetry breaking mechanisms}

The distinction between stable, metastable and unstable films can be made in terms of $\Phi$ as seen in Figure 1.7 .

The black curve characterizes a film that is stable on the substrate, since energy would be necessary to thin the film. The equilibrium film thickness is infinite. The two other curves exhibit a global minimum of $\Phi(h)$ at $h=h^{*}$ and the system can gain energy 


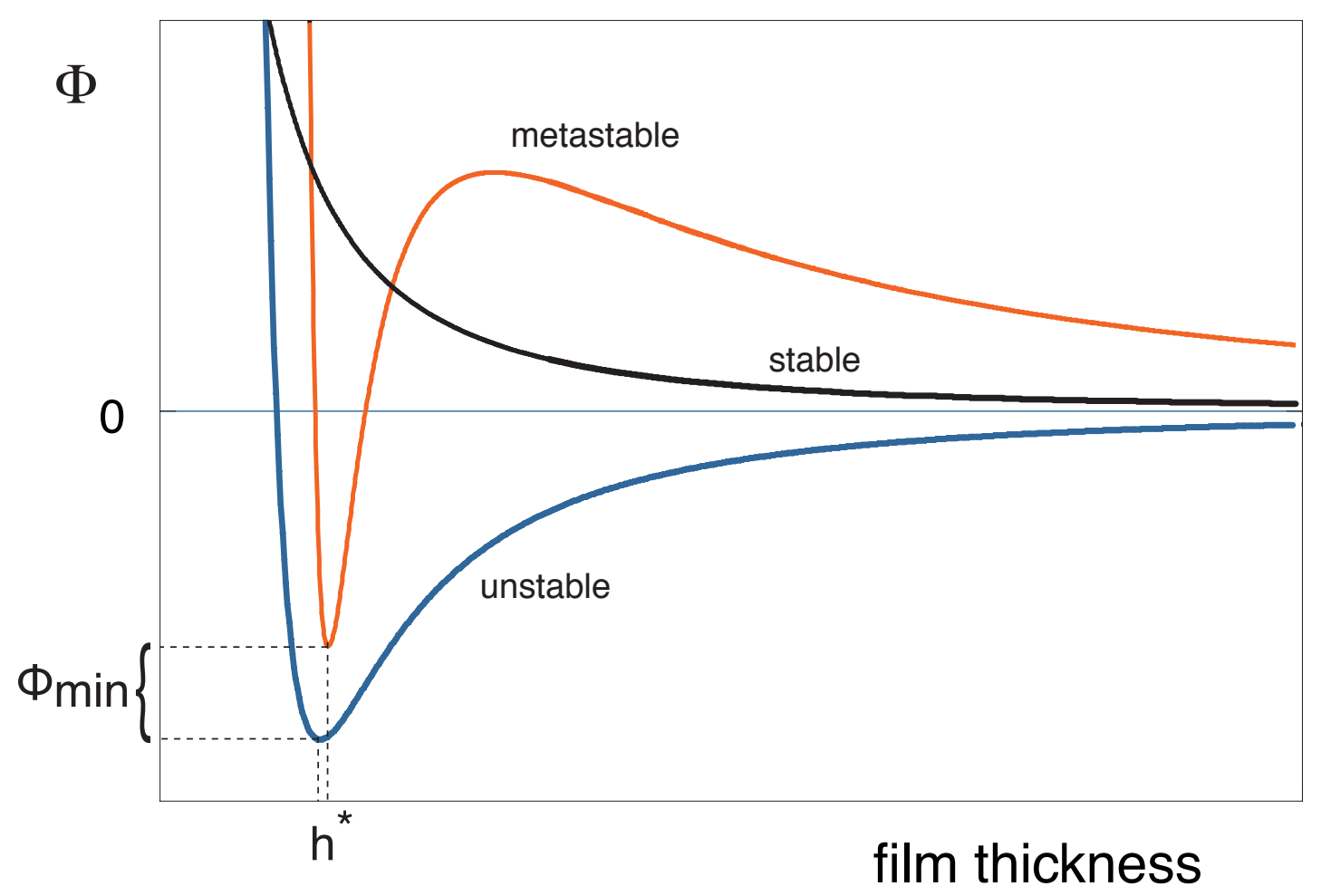

Figure 1.7: Effective interface potential $\Phi$ as a function of film thickness $h$. The black curve corresponds to the stable case, the red one to the metastable and the blue curve corresponds to the unstable case.

by changing its present film thickness $h$ to $h^{*}$. The blue curve characterizes a film that is unstable on the substrate. It is readily shown [25-28] that, if the second derivative of $\Phi(h)$ with respect to $h$ is negative, unstable modes exist, whose amplitudes grow exponentially according to $\exp (t / \tau)$, where $\tau$ is the growth time that is characteristic for the respective mode. Furthermore, there is a characteristic wavelength $\lambda_{s}$ of these modes, the amplitude of which grows the fastest and will therefore dominate the emerging dewetting pattern. This process is analogous to spinodal decomposition of a blend of incompatible liquids, which occurs if the second derivative of the free energy with respect to the composition is negative. There, as well, a certain wavelength exists, the amplitude of which is amplified the strongest. Following this analogy, dewetting via unstable surface waves has been termed spinodal dewetting.

The red curve characterizes a film that is metastable on the substrate. For film thick- 
nesses where the second derivative $\Phi^{\prime \prime}(h)$ is positive, the film is considered metastable, since the system has to overcome a potential barrier in order to reach its state of lowest energy at $h=h^{*}$. Some kind of nucleus, e.g. a dust particle, is required to lower $\Phi(h)$ and can therefore induce dewetting. This rupture mechanism is termed heterogeneous nucleation. For film thicknesses where $\Phi^{\prime \prime}(h)$ is negative and $\left|\Phi^{\prime \prime}(h)\right|$ is increasing with decreasing film thickness, no nucleus is necessary in order for the film to lower its local thickness, as the thermal "activation" is sufficient to overcome the energy barrier (homogeneous nucleation). Finally, for film thicknesses where $\Phi^{\prime \prime}(h)$ is negative and $\left|\Phi^{\prime \prime}(h)\right|$ is decreasing with decreasing film thickness, the film becomes unstable and its decay will proceed via spinodal dewetting.

\section{Linking the effective interface potential to macroscopic properties}

Already in 1938, A. Frumkin had stressed that there is a link between the effective interface potential and the macroscopic contact angle of Young [29], which is given in the following equation:

$$
\frac{\Phi\left(h^{*}\right)}{\gamma_{L V}}=1-\cos (\theta)
$$

Thus, knowing the surface tension $\gamma_{L V}$ and the macroscopic contact angle $\theta$, we can use Equation 1.10 to determine the global minimum of the effective interface potential $\Phi\left(h^{*}\right)$.

Another "macroscopically" accessible quantity that is linked to the effective interface potential is the spinodal wavelength $\lambda_{s}[26,27$ :

$$
\lambda_{s}(h)=\sqrt{\frac{-8 \pi^{2} \gamma}{\Phi^{\prime \prime}(h)}}
$$

The above equation illustrates that only if $\Phi^{\prime \prime}(h)<0$ (spinodal dewetting), is $\lambda_{s}(h)$ real. For $\Phi^{\prime \prime}(h)=0, \lambda_{s}(h)$ diverges to infinity. By determining the spinodal wavelength as a function of the film thickness, one can gain insight into the course of $\Phi^{\prime \prime}(h)$. By measuring the equilibrium layer thickness $h^{*}$ and the contact angle $\theta$, it is possible to reconstruct the complete effective interface potential. An offspring of the reconstruction of the effective interface potential $\Phi$ is that the Hamaker constant $A$ of the system can be determined. The values for $A$ can be compared with the values calculated from optical properties of the media involved. 


\subsubsection{Moving liquid on a rigid substrate - Slippage}

In hydrodynamics, it was typically assumed for convenience that the velocity of a flowing liquid becomes zero at the solid/liquid interface. This means that the liquid molecules adjacent to the solid surface are stationary relative to the solid. Navier was the first to propose [30] that a liquid may slip on a solid surface and this slippage would be opposed by a frictional force that is proportional to the velocity of the fluid relative to the solid and thus introduced the idea of slip length [31 33. The slip length $b$ is the distance to which the velocity extrapolates to zero away from the wall, inside the solid and is related to the velocity of the liquid at the wall $\nu_{r}$ by the Equation 1.12

$$
\nu_{r}=b \frac{\partial \nu_{b}}{\partial z}
$$

where $\nu_{b}$ is the velocity of the fluid in the bulk and $z$ is the axis perpendicular to the wall.

(a)

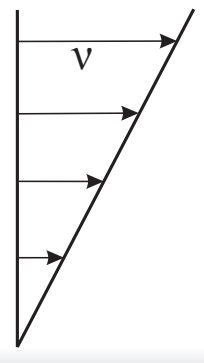

$\mathrm{b}=0$ (b)

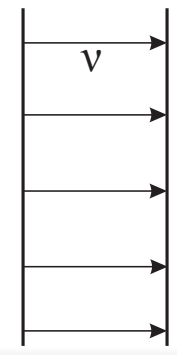

$b=\infty$ (c)

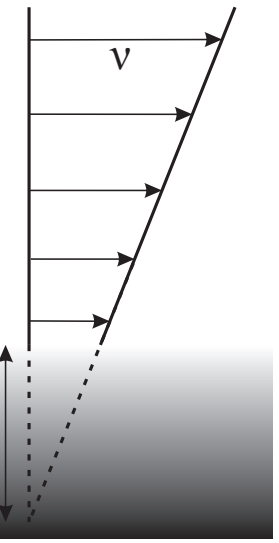

Figure 1.8: Slippage of a thin liquid film on a solid surface. (a) no-slip $b=0$, (b) full-slip with slip length $b=\infty$ and (c) partial-slip with a finite slip length $b$ 
When the velocity of the liquid at the solid/liquid interface is the same as at the liquid/vapor interface, the whole liquid is moving as a plug and the slip length is infinite. This is known as the full-slip boundary condition (Figure 1.8,(b)). The opposite case where the velocity of the liquid at the solid/liquid interface is zero is known as the no-slip boundary condition (Figure 1.8.(a)). The situation where the velocity of the liquid at the solid/liquid interface is non-zero, but nevertheless smaller than that at the liquid-vapor interface, is known as the partial-slip boundary condition (Figure 1.8.(c)) and can be divided into three further cases: the weak, intermediate and the strong slip.

There are several techniques to measure the slip-length in a system, such as Surface Force Apparatus (SFA), Particle Image Velocimetry (PIV), colloidal probe AFM, just to mention some [34]. Recently, a new method has been developed by Fetzer et al. [35], that allows for the extraction of the slip-length by studying the dewetting rim profile (Figure 1.9). Fetzer et al. investigated the dewetting rim profiles for different polymer melts on substrates of different wettabilities. Under the lubrication approximation, the NavierStokes equation was solved with the slip boundary condition and, from the solution, a value for the slip length could be extracted.
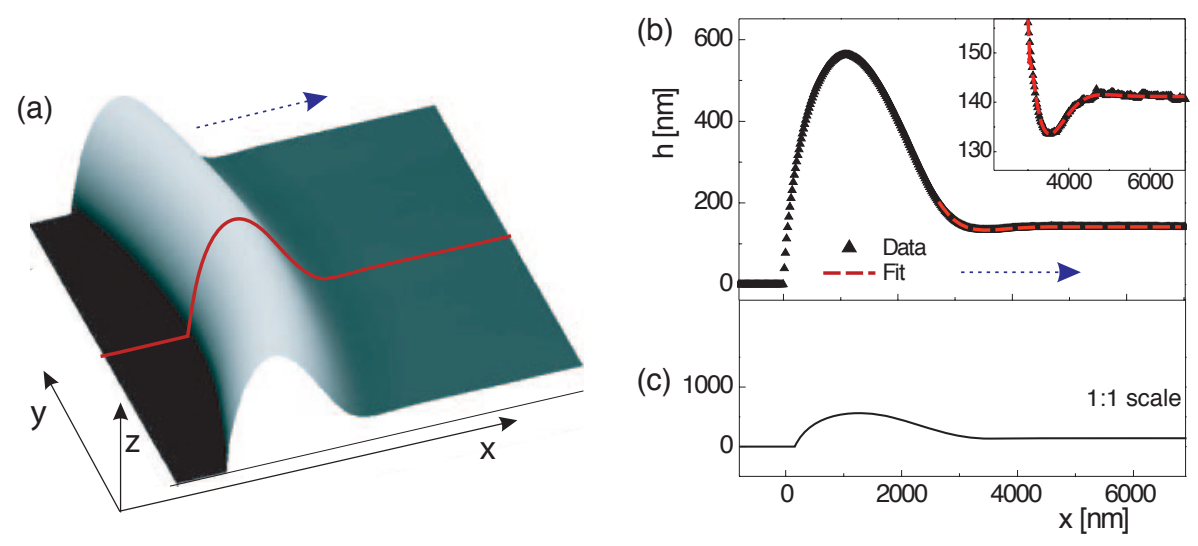

Figure 1.9: Profile of a moving front of a hole. (a) AFM image of a part of a dewetting hole. The dashed arrow indicates the direction of the rim motion. (b) A cross section taken in the radial direction of the rim (red solid line in (a)) gives the rim profile (triangles) which is well fitted by an exponentially decaying oscillation (red dashed line). The inset shows the enlarged view of the oscillation. (c) Data of (b) in a 1 : 1 scale. (Image courtesy of R. Fetzer.) 


\subsection{Wetting of structured substrates}

In the framework of this thesis, we are interested also in the liquid morphologies on topographically structured rubber substrates, subject which will be discussed in Chapter 6. In order to be able to better interpret our experimental findings in the case of the topographically structured rubber substrates, in the present section we will introduce the influence that a non-planar or chemically heterogeneous rigid substrate exerts on the resulting liquid morphology.

\subsubsection{Contact line pinning}
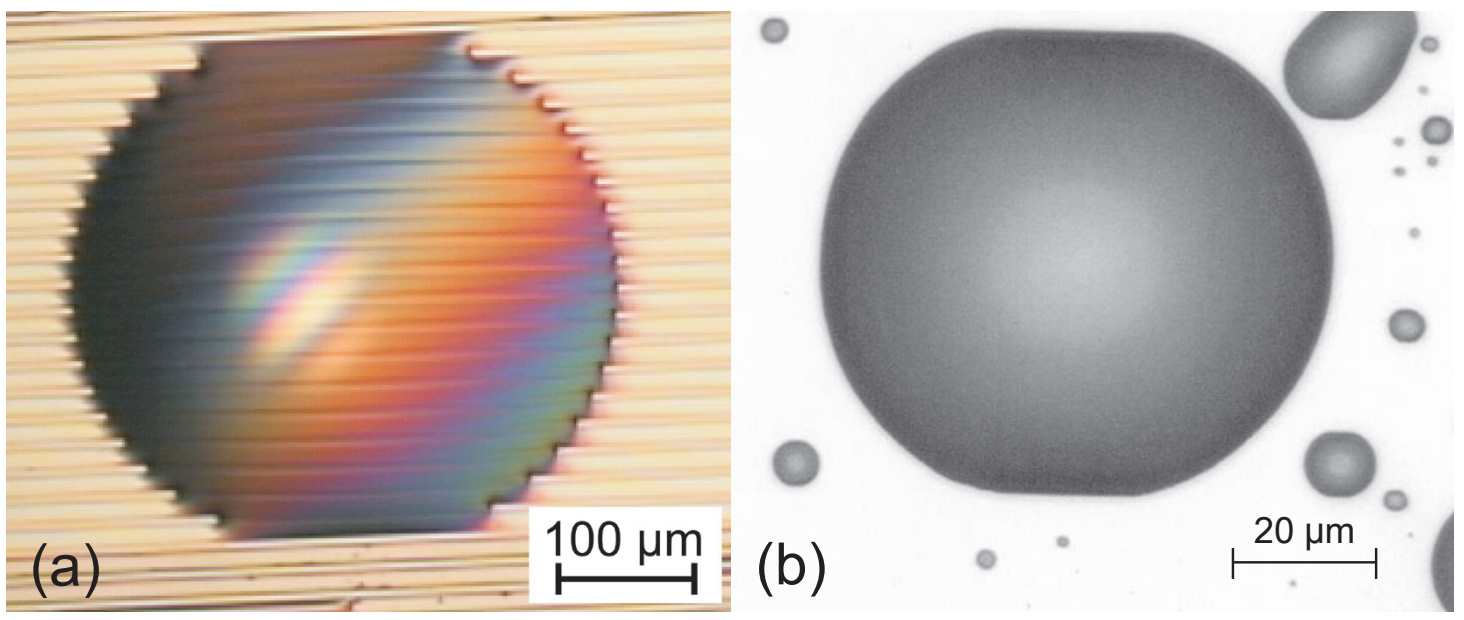

Figure 1.10: Optical micrographs showing (a) a polymer droplet on a topographically structured substrate with rectangular grooves, (b) a hexaethylene glycol droplet deposited on a planar hydrophobic substrate bearing horizontal wettable stripes with a periodicity of $400 \mathrm{~nm}$. In both cases, pinning of the three phase contact line is clearly visible from the non-spherical shape of the droplet's perimeter. (Image courtesy of S. Herminghaus.)

Figure 1.10 depicts two examples of the vast variety of liquid morphologies that can be observed on surfaces with complex geometries, where the three phase contact line of a drop is deformed due to topographical (Figure 1.10.(a)) or chemical (Figure 1.10.(b)) heterogeneities. The key to understanding the large spectrum of different liquid 
morphologies on such surfaces, lies in the behaviour of the three-phase contact line [36[39]. If a surface has a continuously varying topography or chemical heterogeneity, then the contact angle is well defined locally according to Young's equation (Equation 1.2). However, if the substrate has a sharp topography (corner, edge) as in Figure 1.10. (a) or a discontinuous wettability pattern as in Figure 1.10.(b), then the situation is very different.

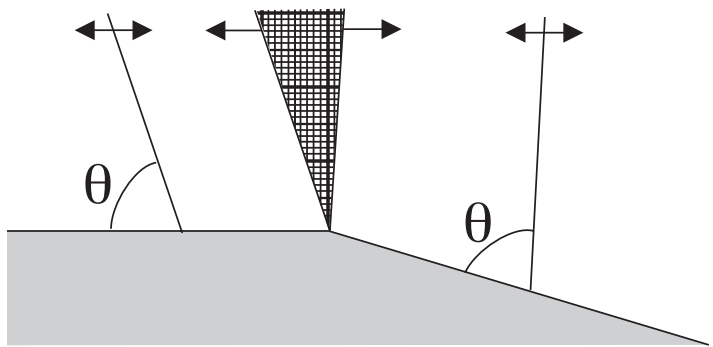

(a)

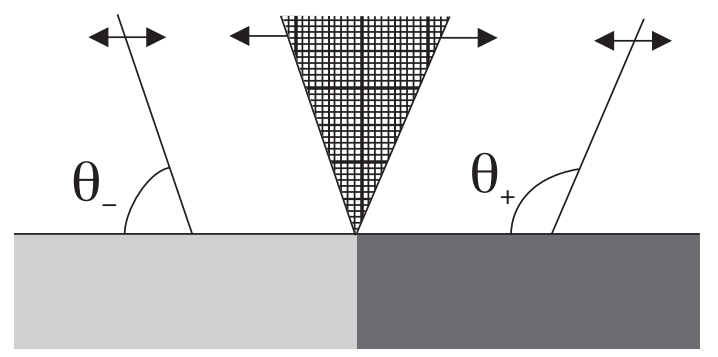

(b)

Figure 1.11: Sketch of a wetting front moving over (a) a topographic step and (b) a wettability step, and corresponding pinning of the three-phase contact line (patterned area).

In this case, Young's equation becomes ill-defined on the discontinuities and the three phase contact line of the liquid can become immobilized due to a big gradient in wettability or an acute edge. The sketch in Figure 1.11.(a) illustrates the pinning of an advancing contact line towards a convex edge on a substrate with homogeneous wettability. Any configuration of the liquid/vapour interface which forms a contact angle larger than the equilibrium contact angle $\theta$ according to Equation 1.2 with respect to the left side of the kink and a contact angle smaller than the contact angle $\theta$ with respect to the right side of the kink can be in equilibrium. Because of its enlarged free range of contact angles, an acute convex edge of the substrate can immobilize the contact line. This effect is also known as the pinning of the contact line. Concave corners, contrary to convex edges, however, cannot immobilize a contact line. Configurations of the liquid/vapour interface with a contact line which is fully or partially aligned with a concave corner of the topography are unstable.

On a planar substrate with sharp wettability patterns (Figure 1.11.(b)), the contact 
angle at the boundary can have any value inbetween the smaller value $\theta_{-}$on the more hydrophilic part and the larger value $\theta_{+}$on the more hydrophobic part. As a consequence, the contact line becomes immobilized and its position gets fixed to the line of discontinuity as long as the contact angle falls into the free range between $\theta_{-}$and $\theta_{+}$.

Hence, the contact angle is not only governed by the local wettability of the substrate, but it also depends on the global shape of the liquid/vapour interface in equilibrium. Therefore, the wettability patterns or the surface topographies may act as "anchoring points" for the contact line of a wetting liquid.

Pinning the contact line by means of surface topographies or chemical steps opens new degrees of freedom for the liquid/vapour interface in equilibrium, as it is responsible for the rich spectrum of static droplet shapes on topographically or chemically structured substrates. But not only the equilibrium is affected by the contact line pinning: the dewetting dynamics of filamentous liquid structures in linear surface grooves is substantially different for laterally pinned and non-pinned contact lines. This phenomenon has strong implications for the liquid pattern after dewetting and for the final droplet morphology [40].

\subsection{Physical properties of polymers}

Over half a century ago, Wofgang Ostwald coined the term the land of neglected dimensions to describe the range of sizes between molecular and macroscopic, within which occur most colloidal particles. The term neglected dimensions could be applied equally well to the world of polymer molecules, the high-molecular-weight compounds so important to man and his modern technology. A polymer is a large molecule built up by the repetition of small, "simple" chemical units. In some cases, the repetition is linear so that a chain is built up from its links. In other cases, the chains are branched or interconnected to form three-dimensional networks. The repeat unit of the polymer is usually equivalent or nearly equivalent to the monomer, the starting material from which the polymer is formed [41].

Our experimental system comprises of polymers. Both the dewetting liquid and the underlying substrate are polymeric, therefore, a good knowledge of the properties of polymers is essential in the context of the research objectives and research approach of 
this thesis. A brief overview of the main physical properties of polymers is presented in the following sections of this chapter.

\subsubsection{Physical states and transitions}

Polymeric materials can be found in a variety of physical states.

- Liquid: Polymer melts and solutions are liquids, but they often are very viscous and show marked viscoelastic properties.

- Crystalline: Polymers can sometimes crystallize, but crystallization is usually not complete, owing to kinetic limitations and the presence of quenched disorder. Familiar examples include synthetic polymers such as polyethylene as well as natural materials like starch.

- Liquid crystalline: Some polymers are rather rigid molecules, which can line up to form liquid crystalline phases. These can form the basis of very strong engineering materials such as Kevlar and liquid crystalls.

- Glass: Because of the difficulty of crystallizing polymers, polymer glasses are very common. Familiar examples include atactic polystyrene, whose arrangement is an advantage in dewetting experiments due to the absence of crystallization effects and its very low ability to take up any humidity from the air.

These physical states depend not only on the processing route taken for the synthesis of the polymer, but also on the given temperature of the polymeric material. Thus, the mechanical response of a polymer depends greatly on temperature. It is impossible to understand the properties of polymers if the transitions that occur in such materials and specifically the temperatures at which these occur, are not known. This strong temperature dependence represents an important characteristic property of polymeric materials and contrasts with the much less sensitive behavior of metals or ceramics.

\section{Glass transition}

The main thermodynamic transitions are the crystalline melting point for the crystalline polymers and the glass transition for the glassy polymers. We are particularly 
interested in the latter, as the transition of polystyrene from the glassy to the liquid state is a mandatory condition in order to observe dewetting. While at relatively high temperatures it is found that relaxation times in liquids, and consequently their viscosities, depend on temperature according to the Arrhenius law, at lower temperatures, assuming that the liquid has not first crystallized, the situation is different. The relaxation time associated with changes in configuration of the atoms or molecules, $\tau_{\text {config }}$, assumes a temperature dependence that deviates strongly from the temperature dependence of the characteristic time of vibrations $\tau_{v i b}$ of the atoms or molecules [42]. This is sketched in Figure 1.12 what we see is that the configurational relaxation time $\tau_{\text {config }}$ appears to diverge at a finite temperature $T_{0}$, the Vogel-Fulcher temperature. Experimentally it is found that the temperature dependence of the relaxation time, and thus the viscosity, follows an empirical law known as the Vogel-Fulcher law [43]:

$$
\eta=\eta_{0} \exp \left[\frac{B}{T-T_{0}}\right]
$$

where $B$ is an emperical constant.

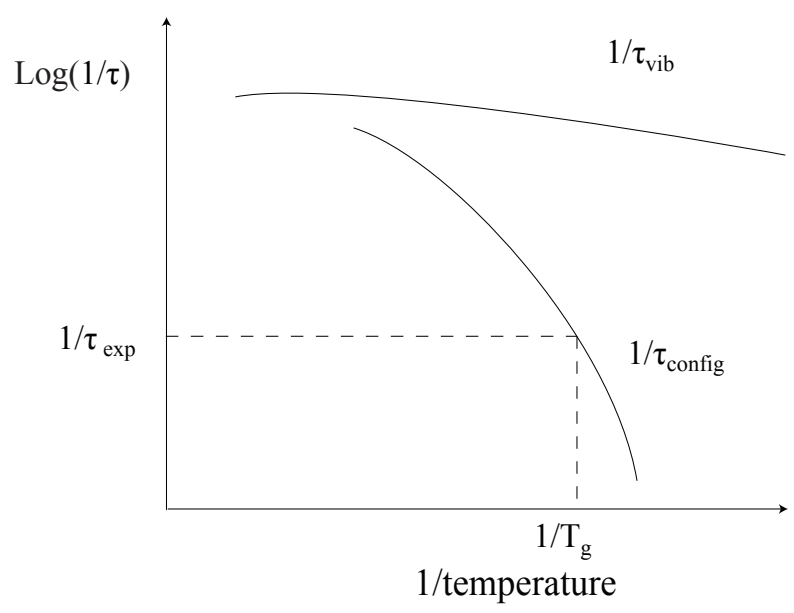

Figure 1.12: Temperature dependence of relaxation times in a glass-forming liquid.

In practice, as the temperature is lowered, we reach a state at which the relaxation time becomes comparable to the timescale of the experiment $\tau_{\text {exp }}$. When this happens, the system falls out of equilibrium with respect to configurational degrees of freedom. This marks the onset of the experimental glass transition, at a temperature $T_{g}$, which is 
called the glass transition temperature.

The glass transition temperature, $T_{g}$, defines a pseudo second-order phase transition in which a glassy solid loses its strength and elasticity and yields a polymer melt or, if it is cross-linked, a rubber. Below the glass transition temperature, $T_{g}$, amorphous solids are in a glassy state [44] and most of their joining bonds are intact. In organic polymers, secondary, non-covalent bonds between the polymer chains become weak above $T_{g}$ and the material becomes soft and capable of plastic deformation without fracture. This behaviour is one of the things that makes most plastics useful for many industrial processes. Nevertheless, such behaviour is not exhibited by crosslinked thermosetting plastics such as the rubber material employed as one of the substrates in our experiments. This type of material, once cured, is "set for life" and will deform elastically under pressure rather than being reshaped, never becoming plastic again when heated, nor melting, even though ageing effects might appear after an extended period of time.

It is well known [45] that the glass transition temperature depends strongly on the molecular weight of the polymer, especially at lower values of molecular weight due to the free volume around the chain ends. The relation between the two is given by the Fox-Flory equation:

$$
T_{g}\left(M_{w}\right)=T_{g}^{\infty}-\frac{K}{M_{w}}
$$

where $T_{g}^{\infty}$ is the glass transition temperature at an infinite molecular weight and $K$ is a constant and they can both be determined experimentally.

\subsubsection{Rheology and mechanical properties of polymers}

In the previous section we introduced the importance of temperature on the mechanical properties of polymeric material. In order to better understand, explain and predict the behaviour of our system during dewetting, we need to classify these mechanical properties. Typical condensed matter comes in two forms: solid and liquid. "Soft condensed matter" is more difficult to categorize. Let us think of glue, soap, tomato ketchup or pastes, are they solid or liquid? In a certain way they seem to have attributes of both. In this section we will first review the ideal behaviour of normal solids and liquids and we will then discuss some of the more common types of non-ideal behaviour.

In Figure 1.13 we can see how shear stress and shear rate are defined. The shear stress $\sigma$ is given in terms of the applied force $F$ and the area $A$ as $\sigma=F / A$, while the 
shear strain $\epsilon$ is given as $\epsilon=\Delta x / y$.

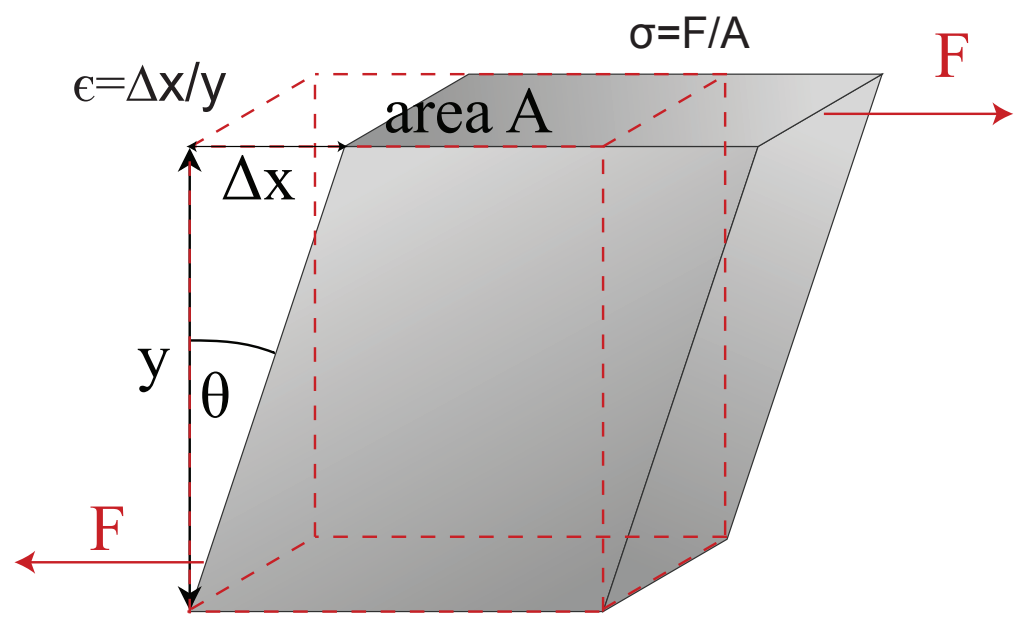

Figure 1.13: A shear stress $\sigma$ is applied to the top of the cube, while the bottom is held in place. This stress results in a deformation or strain $\epsilon$, changing the cube into a parallelepiped.

For a solid, the application of a stress to the material produces a constant strain in response; if the strain is simply proportional to the applied stress, then the solid is called a Hookean solid with the constant of proportionality being the elastic modulus $\lambda$. Thus:

$$
\lambda=\frac{\sigma}{\epsilon}
$$

For a liquid, a constant applied stress will result in a time-dependent strain. If the strain rate is constant when a constant stress is applied, then the liquid is called a Newtonian liquid. In the elementary definition of viscosity, we imagine some liquid sandwiched between parallel plates of area $A$ separated by a distance $y$. If the plates are moved with relative velocity $u$, then the force $F$ resisting the relative motion of the plates is given by:

$$
F=A \eta \frac{u}{y}
$$

where the coefficient $\eta$ is the viscosity of the fluid. The velocity gradient $u / y$ is in fact identical to the time derivative of the shear strain $\dot{\epsilon}$, so we can write this definition in a 
more general form as:

$$
\sigma=\eta \dot{\epsilon}
$$

Summarizing these two types of behaviour:

\section{Hookean solid}

- An applied shear stress produces a shear strain in response.

- The shear strain is proportional to the shear stress and the constant of proportionality is the elastic modulus.

\section{Newtonian liquid}

- An applied shear stress produces a flow with a constant shear strain rate in response.

- The shear strain rate is proportional to the shear stress and the constant of proportionality is the viscosity.

\subsubsection{Elastic modulus}

In the definition of a Hookean solid, the constant of proportionality between strain and stress is the elastic modulus $\lambda$. Depending on how stress and strain are measured, we can define several types of elastic moduli. The three primary ones are:

- Young's modulus E; describes tensile elasticity or the tendency of an object to deform along an axis when opposing forces are applied along that axis; it is defined as the ratio of tensile stress to tensile strain. It is often referred to simply as the elastic modulus.

- The shear modulus or modulus of rigidity $G$ describes an object's tendency to shear (the deformation of shape at constant volume) when acted upon by opposing forces; it is defined as shear stress over shear strain. The shear modulus is part of the derivation of viscosity. 
- The bulk modulus $K$ describes volumetric elasticity or the tendency of an object's volume to deform under pressure; it is defined as volumetric stress over volumetric strain and is the inverse of compressibility. The bulk modulus is an extension of Young's modulus to three dimensions.

For homogeneous isotropic materials, simple relations exist between elastic constants (Young's modulus $E$, shear modulus $G$, bulk modulus $K$, and Poisson's ratio $\nu$ ) that allow calculating them all as long as two are known:

$$
E=2 G(1+\nu)=3 K(1-2 \nu)
$$

\subsubsection{Viscoelasticity}

So far we have defined two simple limiting behaviours for condensed matter: the elastic Hookean solid, and the viscous Newtonian liquid. Real materials and specifically polymers, at one extreme, they behave like elastic solids; when an external stress is released, the material snaps back to its undeformed state and energy spent on deforming the polymer is recovered. At the other extreme, polymers exhibit viscous flow accom-

panied by energy dissipation in response to stress. In general though, the response of a polymer to a stress is characterized by both viscous and elastic components with an additional dependence on timescale, so that their mechanical behaviour is described as viscoelastic.

A famous experiment that demonstrates the dual nature of the viscoelastic substances is the pitch drop experiment shown in Figure 1.14. It is a long-term experiment that started at the University of Queensland in Brisbane, Australia in 1927 and is still progressing. Its aim is to demonstrate that some substances that appear to be solid are in fact very-high-viscosity fluids. The experiment consists in a piece of pitch that flows extremely slowly through a funnel. For each droplet to form and fall about a decade is required.

Another example of this kind of behaviour can be observed in the material "silly putty", available in toy shops. If one applies a stress on a slow timescale, this material flows like a very viscous liquid, but if one rolls it up into a ball and drops it on a hard surface, it bounces back elastically.

In Figure 1.15 we imagine a stress being applied at time $t=0$ and held constant. 


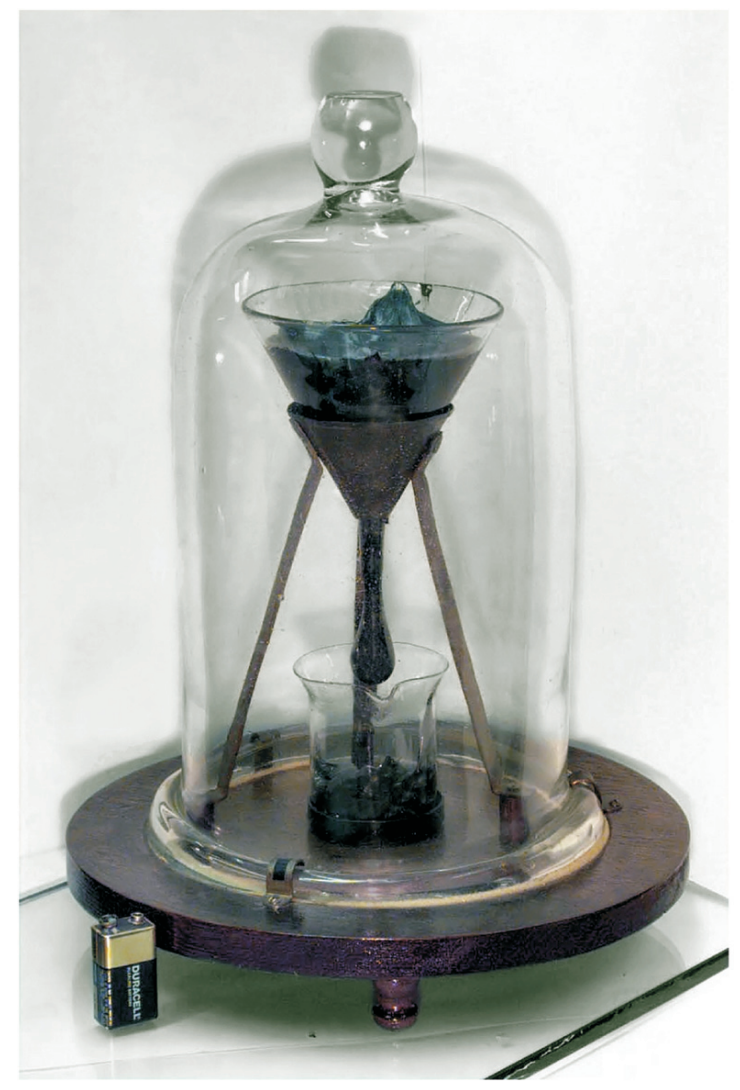

Figure 1.14: Pitch drop flowing through a funnel, University of Queensland in Brisbane, Australia.

The material responds at first in an elastic way, with a constant strain, but, after a certain time $\tau$, it begins to flow like a liquid, with the strain increasing linearly with time. The time $\tau$ is the relaxation time; it is the time that separates the solid-like from the liquid-like behaviour. If a stress is applied on a timescale that is shorter than the relaxation time, the material will behave like a solid. For stresses that are applied on longer timescales than the relaxation time, the viscoelastic material flows. Another way in which complex fluids can display departures from the ideal Newtonian fluid behaviour is in having an effective viscosity which depends on shear rate. In this case, for a steady flow, we can generalize Equation 1.17 to:

$$
\sigma=\eta(\dot{\epsilon}) \dot{\epsilon}
$$




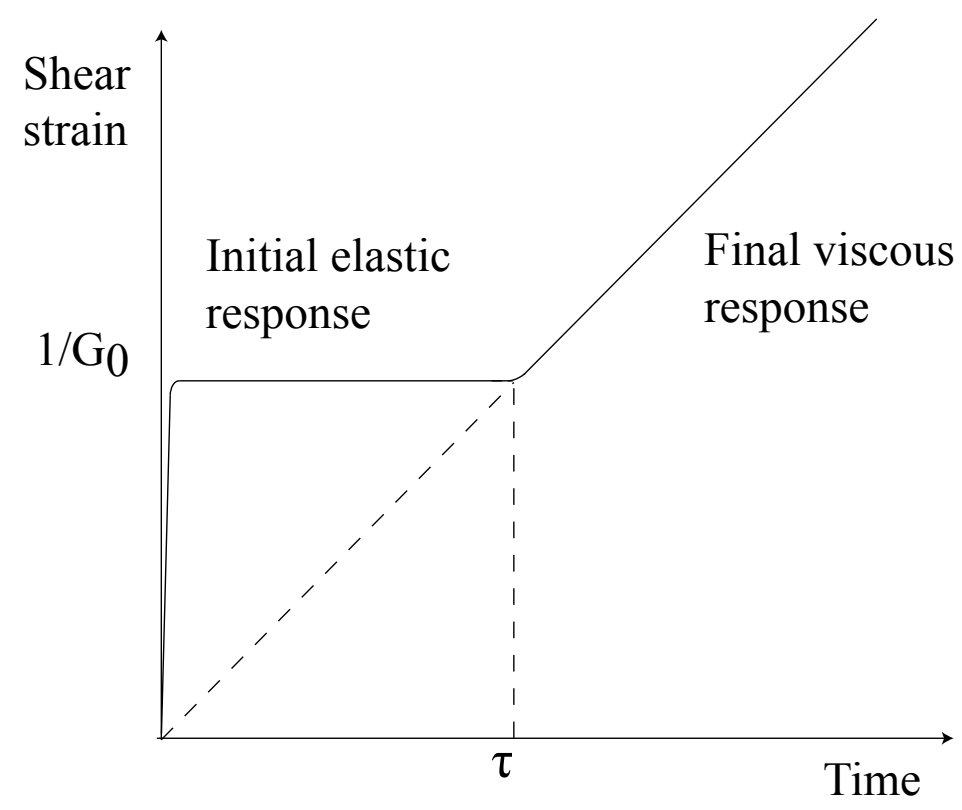

Figure 1.15: Schematic strain response of a viscoelastic material to a shear stress applied at time $t=0$ and subsequently held constant.

where $\eta(\dot{\epsilon})$ is shear-rate dependent viscosity.

Possible responses of a fluid to an applied stress are sketched in Figure 1.16. In contrast to a Newtonian fluid (Figure 1.16.(a)), a shear-thinning fluid (Figure 1.16.(b)) becomes progressively easier to make flow as the shear rate gets larger. This is for example a desirable behaviour for the ink in a ballpoint pen and for a paint that should be easy to apply at the relatively high shear rates achieved by brushing, but, as a thin film applied to a vertical surface, it should not sag under its own weight. A shear thickening fluid (Figure 1.16.(c)), on the other hand, flows relatively easily when a low shear rate is applied but becomes much more resistant to flow when sheared at a high rate. This behaviour is often seen in pastes with a rather high volume fraction of particles.

Liquid polymers are employed throughout this thesis not only in the role of the dewetting liquid, but also as substrates. Above a critical polymer chain length, which is called entanglement length and varies from polymer to polymer, there exist ropelike intermolecular couplings at a number of points along the length of the molecule, which cause non-linear flow behaviour and make the studying of dewetting phenomena significantly more complicated. Therefore, throughout this thesis, we always make a 


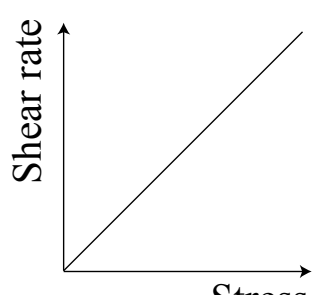

Stress

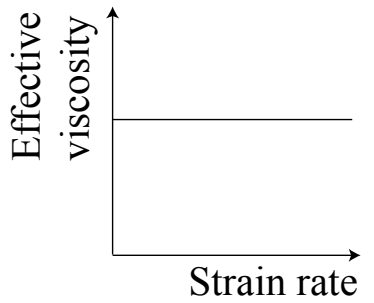

(a)
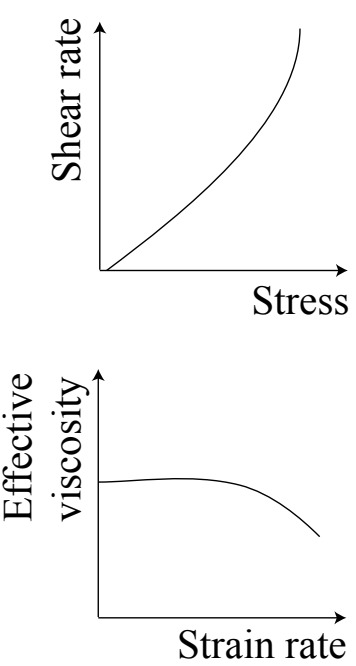

(b)
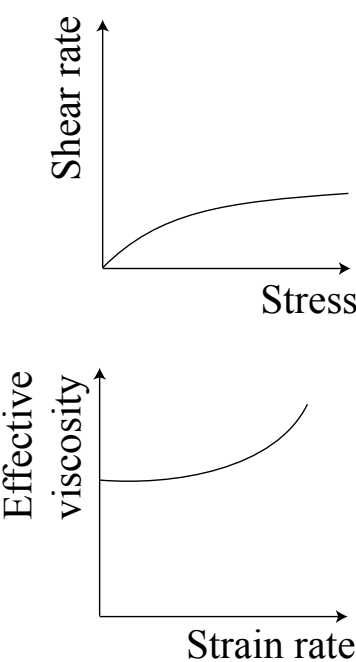

(c)

Figure 1.16: Some possible responses of a fluid to an applied stress: (a) Newtonian, (b) Shear thinning, (c) Shear thickening.

distinction if the chain-length of the polymer used is above or below the entanglement length. In the latter case, the polymer can be considered to behave as a Newtonian liquid.

\section{Elastomers}

One of the deformable substrates employed in our experiments is an elastomer called Poly(dimethylsiloxane) (PDMS). An elastomer is a polymer melt in which cross-links, randomly placed between adjacent chains, bond the chains together to form a macroscopic network. At a local level, the material behaves like a liquid; in particular the bulk modulus is rather high and, to a first approximation, the material may be taken as incompressible. However, the cross-links mean that macroscopic bulk flow cannot take place and the material has a finite shear modulus. The properties of typical elastomers are defined by the following requirements:

- They must stretch rapidly and considerably under tension, reaching high elongations (500-1000\%) with low damping, that is, little loss of energy as heat.

- They must exhibit high tensile strength and high elastic modulus (stiffness) when fully stretched. 
- They must retract rapidly, exhibiting the phenomenon of snap or rebound.

- They must recover their original dimensions fully on the release of the stress, exhibiting the phenomena of resilience and permanent set close to zero. That means that the energy absorbed when deformed should be fully recovered upon unloading. In parallel, the deformation remaining after the elastomer has been deformed for a definite period and then released should approach zero.

Although the thermodynamics associated with rubber elasticity was developed in the middle of the nineteenth century, the molecular requirements for the exhibition of rubbery behaviour were not recognized until 1932. Theories of the mechanism relating these molecular-structure requirements to the phenomena of rubber elasticity were developed soon after. The molecular requirements of elastomers may be summarized as follows:

- The material must be a "high polymer", i.e. a polymer with more than 100 repeat units.

- It must be above its glass transition temperature $T_{g}$ to obtain high local segment mobility.

- It must be amorphous in its stable (unstressed) state for the same reason.

- It must contain a network of crosslinks to restrain gross mobility of its chains.

\section{Stress-strain behaviour of elastomers}

The stress-strain curve of a typical elastomer in Figure 1.17 shows marked deviations from the straight line required by Hooke's law. The relatively low slope of the curve decreases to about one third of its original value over the first hundred percent elongation and later increases, often to quite high values at high elongations. A simple model that explains this behaviour was proposed by Guth in 1946 [46]. In his model, Guth assumes that the actual tangled mass of polymer chains may be represented by an idealized network of flexible chains, irregular in detail, but homogeneous and isotropic, extending

throughout the sample. The network consists of $m$ chains of average length $\sqrt{r^{2}}$ per unit volume directed along each of the three perpendicular axes. To obtain the proper 


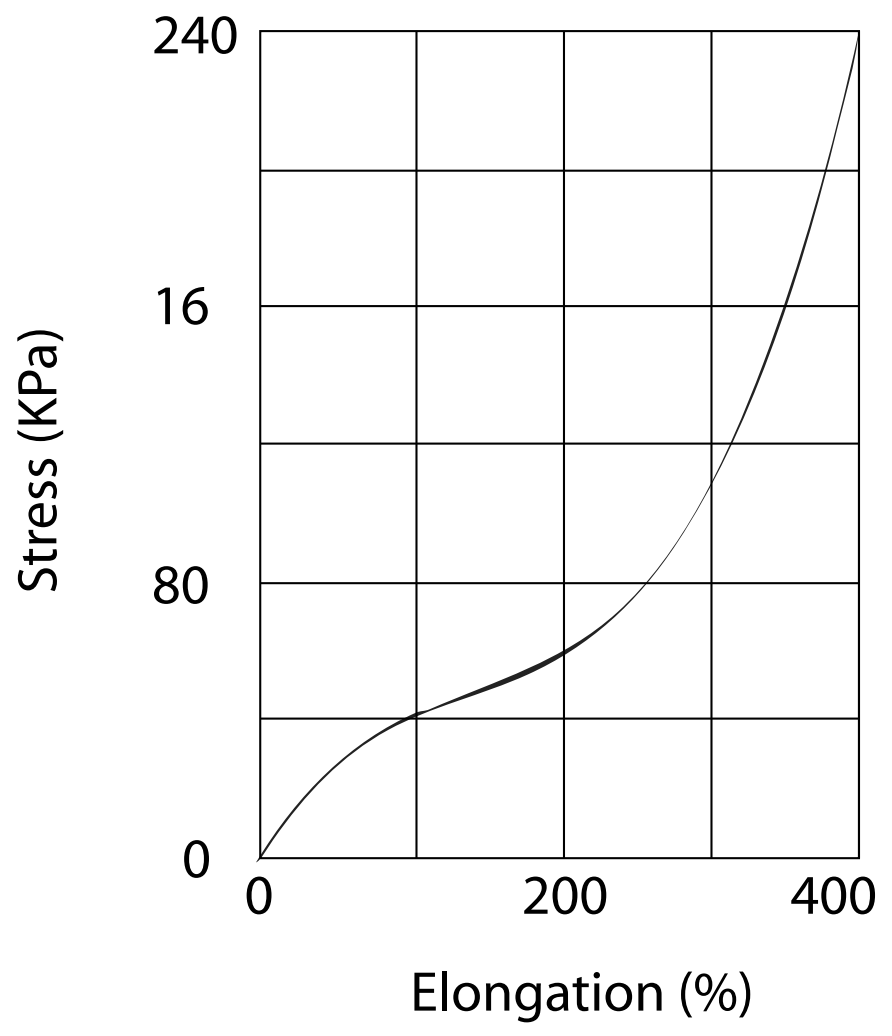

Figure 1.17: Stress-strain curve for a typical elastomer.

space-filling properties of the model, it is assumed that the space between the chains is filled with an incompressible fluid exerting a hydrostatic pressure $p$ outwards against the elastic tension of the chains. If we consider the equilibrium of inward and outward forces when a cube of this material is stretched into a parallelepiped, it can be shown that the stress $\sigma$ is related to the strain $\epsilon$ by:

$$
\sigma=2 m k T b^{2}\left[\epsilon-\frac{1}{e^{2}}\right]
$$

where $k$ is Boltzmann's constant and $b^{2}=\frac{3}{2} x l^{2}, x$ being the number of links with length 
$l$. The shear modulus is given then by:

$$
G=\frac{d \sigma}{d \epsilon}=2 m k T b^{2}\left[1+\frac{2}{\epsilon^{3}}\right]
$$

A simple physical interpretation of the rate dependence of mechanical response in viscoelastic systems can be devised in terms of a mechanical model involving springs and dashpots that recalls that of electric circuits with resistance and inductance. Before attempting to propose a model that explains the viscoelastic behaviour of an actual polymer, it is advisable to examine the simplest configuration which comprises one spring that obeys Hooke's law and one dashpot corresponding to a Newtonian liquid.

The two elements of spring and dashpot can be basically combined in two ways.

\section{Maxwell Model}

If spring and dashpot are placed in series, the resulting Maxwell element exhibits flow plus elasticity on the application of stress.

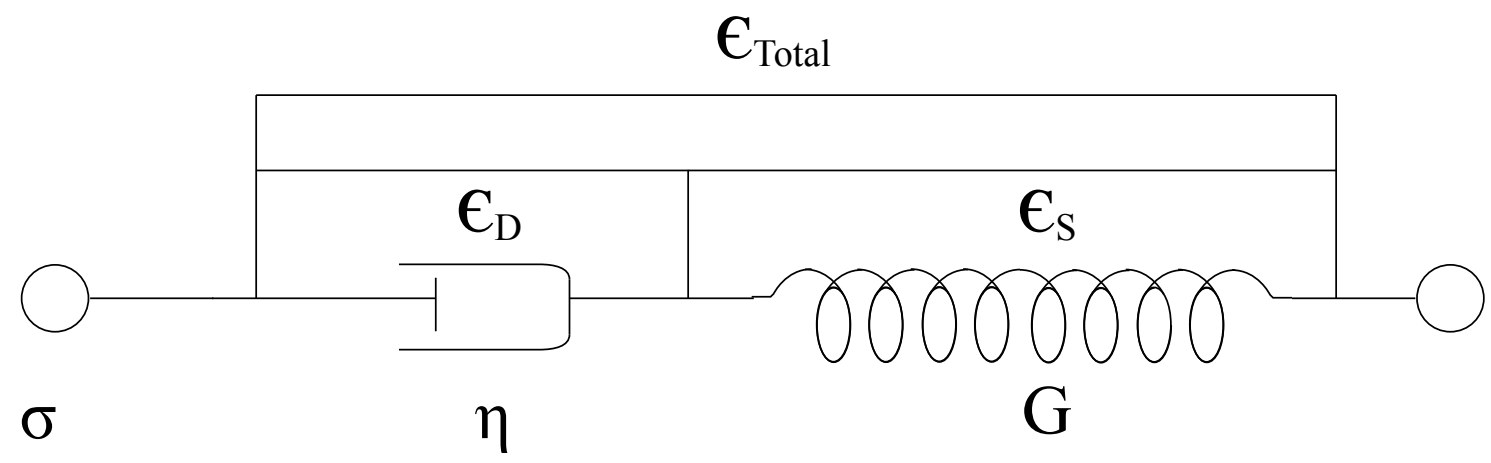

Figure 1.18: Schematic representation of a Maxwell element.

In this configuration, under an applied axial stress, the total stress, $\sigma_{\text {Total }}$ and the total strain, $\epsilon_{\text {Total }}$ can be defined as follows [41]:

$$
\begin{gathered}
\sigma_{\text {Total }}=\sigma_{D}=\sigma_{S} \\
\epsilon_{\text {Total }}=\epsilon_{D}+\epsilon_{S}
\end{gathered}
$$

where the subscript $D$ indicates the stress/strain in the dashpot and the subscript $S$ 
indicates the stress/strain in the spring. When the stress is applied, the spring elongates, while the dashpot slowly yields. On the removal of the stress, the spring recovers, but the dashpot does not. Taking the derivative of strain with respect to time, we obtain:

$$
\frac{d \epsilon_{\text {Total }}}{d t}=\frac{d \epsilon_{D}}{d t}+\frac{d \epsilon_{S}}{d t}=\frac{\sigma}{\eta}+\frac{1}{G} \frac{d \sigma}{d t}
$$

or, in dot notation:

$$
\dot{\epsilon}=\frac{\sigma}{\eta}+\frac{\dot{\sigma}}{G}
$$

where $G$ is the shear modulus and $\eta$ is the material coefficient of viscosity. This model describes the damper as a Newtonian fluid and the spring as a Hookean solid.

The relation of creep to stress relaxation may be seen by considering the experiment in which a strain is obtained and then held by fixing the ends of the system, i.e. $\dot{\epsilon}=0$ in Equation 1.25. The equation can then be solved:

$$
\sigma=\sigma_{0} e^{-\frac{G}{\eta} t}=\sigma_{0} e^{-\frac{t}{\tau}}
$$

where the stress $\sigma$ relaxes from its initial value $\sigma_{0}$ exponentially as a function of time. The time $\eta / G$ after which the stress reaches $1 / e$ of its initial value is the relaxation time $\tau$.

The equation can be applied either to the shear stress or to the uniform tension in a material. In the former case, the viscosity corresponds to that of a Newtonian fluid. In the latter case, it has a slightly different meaning relating stress and rate of strain.

The model is usually applied to the case of small deformations.

\section{Kelvin-Voigt Model}

Most polymers do not exhibit the unrestricted flow permitted by the Maxwell model, although it might be a reasonable model for Silly Putty or warm tar. For more typical polymers, whose conformational change is eventually limited by the network of entanglements or other types of junction points, their response to a stress is better represented by a parallel combination of spring and dashpot in a Kelvin-Voigt element.

This element shows a retarded elastic or viscoelastic response. The dashpot acts as a damping resistance to the establishment of the equilibrium of the spring. Since the 


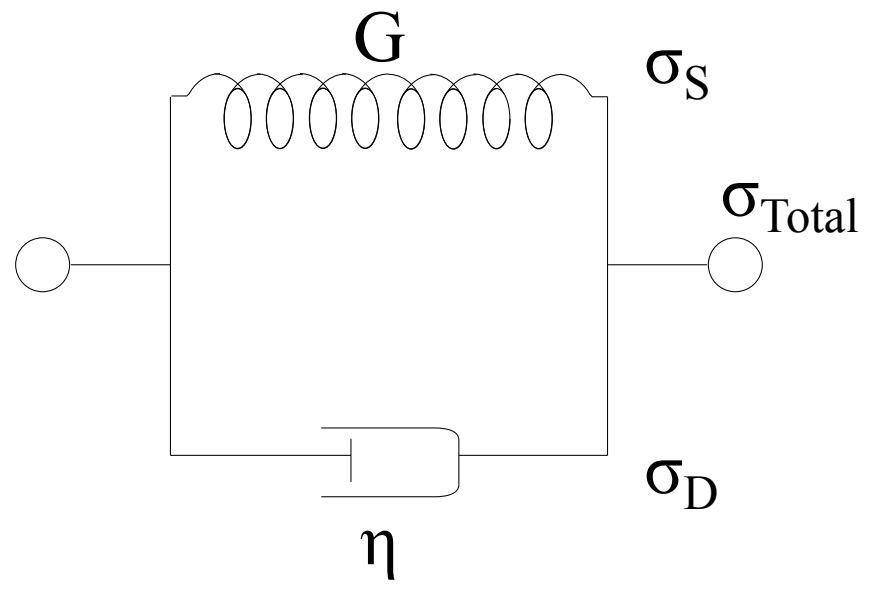

Figure 1.19: Schematic representation of a Kelvin-Voigt element.

two components of the model are arranged in parallel, the strains in each component are identical:

$$
\epsilon_{\text {Total }}=\epsilon_{D}=\epsilon_{S}
$$

Similarly, the total stress will be the sum of the stress in each component:

$$
\sigma_{\text {Total }}=\sigma_{D}+\sigma_{S}
$$

The equation for the stress is:

$$
\sigma(t)=\eta \frac{d \epsilon(t)}{d t}+G \epsilon(t)
$$

where $G$ is the shear modulus and $\eta$ is the viscosity. The equation can be applied either to the shear stress or to the normal stress of a material.

If a stress is applied and removed after some time, the deformation curve is given by

$$
\epsilon=\frac{\sigma}{G}\left(1-e^{-\frac{G}{\eta} t}\right)=\frac{\sigma}{G}\left(1-e^{-\frac{t}{\tau}}\right)
$$

where $\tau$ is a retardation time. When the stress is removed, the sample returns to its original shape along the exponential curve:

$$
\epsilon=\epsilon_{0} e^{-\frac{t}{\tau}}
$$


Summarising, the relaxation times for the basic models are shown schematically in Figure 1.20 .

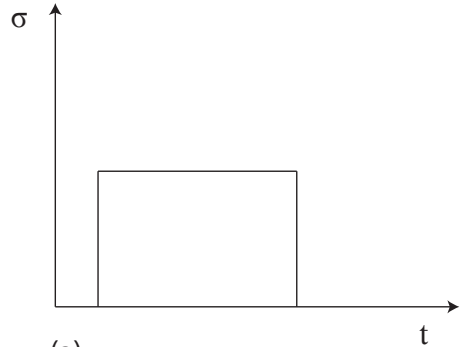

(a)

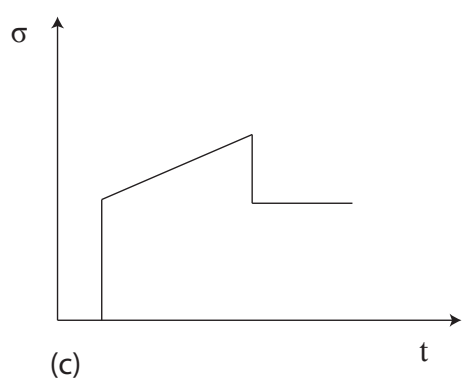

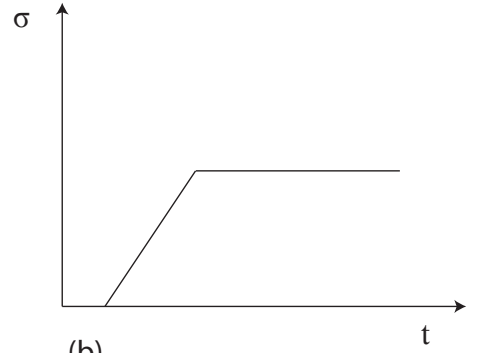

(b)

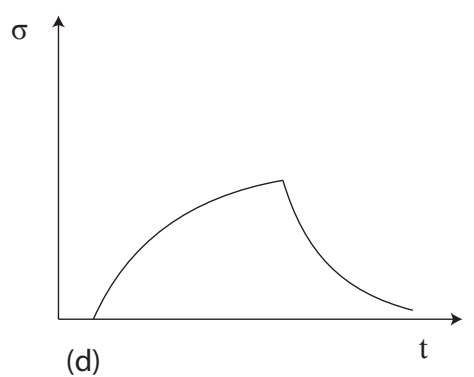

Figure 1.20: Strain-time relationships at constant stress for simple models: (a) Hookean solid (ideal elastic spring), (b) Newtonian fluid (dashpot), (c) Maxwell element, (d) Kelvin-Voigt element

\section{Standard Linear Solid Model (SLS)}

Placing a spring in parallel with a Maxwell element gives a very useful model that describes the overall behaviour of a system under a given set of loading conditions, known as the Standard Linear Solid (S.L.S.) shown in Figure 1.21.

In this arrangement, the Maxwell arm, which contains a spring $\left(G=G_{2}\right)$ and a dashpot (viscosity $\eta$ ) in series, and the parallel spring $\left(G=G_{1}\right)$ experience the same strain $\epsilon$, whereas the total stress $\sigma$ is the sum of the stress in each arm: $\sigma_{\text {Total }}=$ $\sigma_{S}+\sigma_{\text {Maxwell }}$.

Although the SLS model is capable of describing the general features of viscoelastic relaxation, being a three-parameter model, its ability to fit the experimental data over the full range of the relaxation is usually poor. 


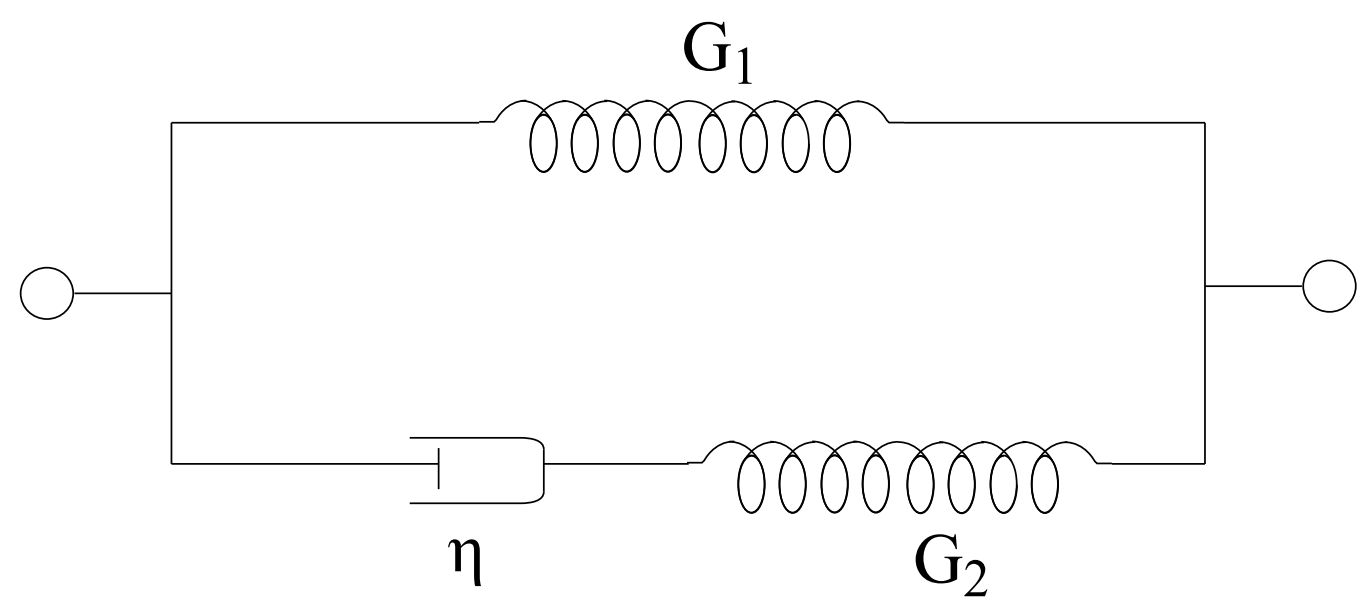

Figure 1.21: Schematic representation of a Standard Linear Solid model.

\section{Basic generalized models}

The components that make up the simplest behaviour of an actual polymer sample in creep can be represented by a mechanical model which combines one Maxwell and one Kelvin-Voigt element in series. If a stress is suddenly applied to this model, the strain changes with time. The model departs from the initial conditions at time $t_{1}$ by an elastic deformation $\sigma / G_{1}$. A viscoelastic response approaching $\sigma / G_{2}$ as an equilibrium value and a viscous flow at rate $\sigma / \eta$. On the removal of the stress at time $t_{2}$, the elastic element relaxes "immediately" and the viscoelastic one slowly, but the viscous flow is never recorded.

Alternatively, the creep and stress-relaxation experiments can be described with a generalized model consisting of one Maxwell and one Kelvin-Voigt element arranged in parallel. The two generalized models are entirely equivalent.

Although these models exhibit the main characteristics of the viscoelastic behaviour of polymers, they are nevertheless oversimplified, since the flow of the polymer is most probably not ideally Newtonian and its elastic behaviour might not be strictly Hookean. 


\section{Generalized Maxwell Model}

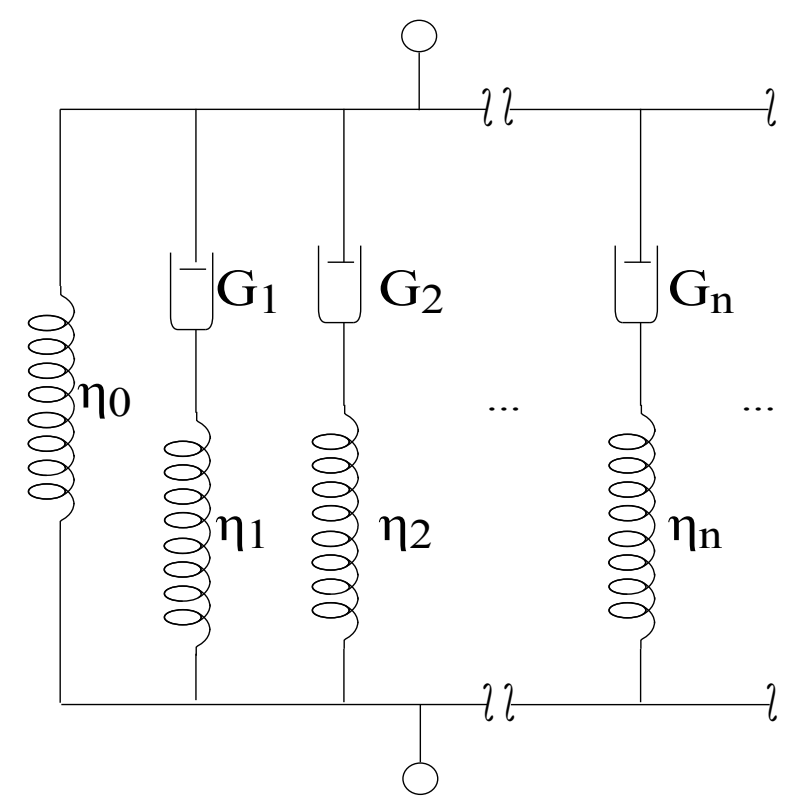

Figure 1.22: Schematic representation of a generalized Maxwell model.

The Generalized Maxwell model (Figure 1.22 ), also known as the Maxwell-Weichert model, is the most general form of the linear model for viscoelasticity. It takes into account that the relaxation does not occur at a single time, but at a distribution of times. Due to molecular segments of different lengths with shorter ones contributing less than longer ones, there is a varying time distribution. The Generalized Maxwell model shows this by having as many spring-dashpot Maxwell elements as necessary to accurately represent the distribution. In practice, the more relaxation times a model considers, the better it represents the behaviour of real polymers, which cannot be characterized by discrete relaxation times, but require a spectrum of relaxation times to account for all phases of their behaviour. 



\section{Chapter 2}

\section{Sample preparation and system characterisation}

For every experimental investigation, the sample preparation marks the first prominent step before proceeding to the actual measurement we are interested in. For dewetting experiments, the cleaning of the underlying substrate and the preparation of the initially homogeneous polymeric film are of great importance. In the first half of this chapter, all techniques used for the preparation of our samples will be introduced and fabrication details will be presented. In the second half, we discuss the different experimental techniques adopted for the characterization of the prepared sample and the execution of the dewetting experiments. The discussion involves an introduction to the physical law on which the technique is based and the corresponding operation principle.

\subsection{Sample Preparation}

We start the description of our preparation methods with the planar viscoelastic substrates. In order to be able to tune independently both the elasticity and the viscosity of the underlying substrate, we use two different types of substrates: rubber substrates, in which we tune the elasticity and liquid substrates in which we tune the viscosity. Subsequently, the preparation of topographically structured rubber elastic substrates will be discussed. 


\subsubsection{Planar viscoelastic substrates}

In Figure 2.1 we show schematically a typical system employed in our experiments. It consists of three layers: The bottom layer is an approximately $(1 \times 1) \mathrm{cm}^{2}$ Silicon wafer. Above lies the viscoelastic substrate, which is either a rubber or a liquid. Finally, on top of it is placed the polystyrene (PS) film to be dewetted. The detailed procedure of the realization of such samples will be presented in the following sections, both for the rubber and for the liquid type of substrates.

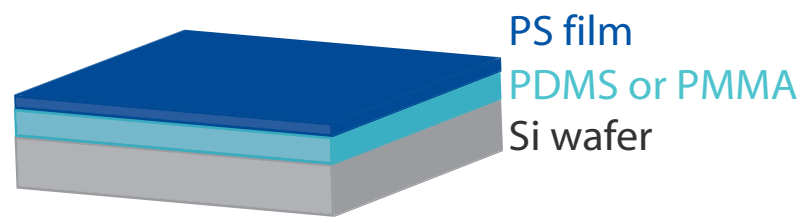

Figure 2.1: A typical sample used in our experiments: the lower layer is a piece of Silicon wafer. The intermediate layer is the viscoelastic substrate (rubber or liquid) and the upper layer is the dewetting polymer film.

\section{Silicon (Si) Wafer Preparation}

The basic purpose of the cleaning is the removal of surface contamination and the establishing of a reproducible and well defined surface. On silicon ( $\mathrm{Si}$ ) substrates, as received from the manufacturer, the top oxide layer is hydrophilic or hydrophobic, depending on the chemicals used during the polishing of the surface. In addition, frequently, contaminations from dust particles or the laboratory environment are present. Without a substrate cleaning, arbitrary results regarding the dewetting of a polymer film are to be expected.

We use planar $\mathrm{Si}$ wafers with a native silicon dioxide layer $(\sim 1 \mathrm{~nm})$ as supporting substrates onto which the actual viscoelastic substrates are placed. The Si wafers (Crystec, Germany) are p-type $<100>$ (dopant Boron) with resistivity $1 \sim 20 \Omega \mathrm{cm}$. The Si wafer is firstly cut into pieces of approximately $(1 \times 1) \mathrm{cm}^{2}$, which are subsequently cleaned by snow-jet (stream of $\mathrm{CO}_{2}$ crystals), a cleaning process that removes micron and submicron particulates and hydrocarbon-based contamination. Next, they are put in a 
Harrick oxygen plasma cleaner and treated for 30 seconds in order to oxidize and remove organic depositions from the surface. Subsequently, they are sonicated successively in solvents of decreasing polarity, i.e. Ethanol, Acetone and Toluene for 3 minutes respectively and dried with compressed nitrogen gas in between changing solvents. Finally, the Si wafers are put again in the plasma cleaner and treated for 30 seconds.

After cleaning the Si wafer, the next step is the preparation of the viscoelastic layer, which, placed on top of the Si wafer, will form the substrate onto which the PS thin film will be deposited.

\section{Rubber substrates}

The rubber viscoelastic substrates used in our experiments consist of cross-linked Poly-dimethylsiloxane (PDMS). Some physical and chemical attributes that make PDMS a suited material for our studies are the low glass transition temperature $\left(T_{g}=-125^{\circ} \mathrm{C}\right)$ and its unique flexibility (the elastic modulus may vary between $300 \mathrm{kPa}$ and $10 \mathrm{MPa}$ ) 47].

The PDMS employed in our study is prepared from SYLGARDR184 (SG184) and SYLGARD®186 (SG186) (Dow Corning) purchased from SASCOHOLZ (Frankfurt, Germany). Both SG184 and SG186 consist of a "base" and a "curing agent" that are typically mixed in a 10:1 w/w ratio. After degassing in vacuum for 15 min., the PDMS is spin-coated on cleaned Si wafers to produce PDMS films with a thickness of $6 \pm 0.5 \mu \mathrm{m}$. The PDMS is subsequently cured in a protective atmosphere $\left(\mathrm{N}_{2}\right)$ at $170{ }^{\circ} \mathrm{C}$ for one hour. The value for the film thickness was chosen after considering the following: since PDMS is a thermal insulator, it should be as thin as possible in order to avoid any thermal gradients during the annealing of the overlying film. On the other hand, the PDMS film had to be thick enough so that it could be safely considered semi-infinite with the presence of the underlying Si wafer not affecting the dewetting phenomena. Finally, the spin-coating parameters resulting in the specific film thickness also had to be chosen such that the roughness of the resulting film would be optimal.

Before degassing, the highly viscous mixture of SG186 is further diluted with toluene in a 1:1 volume ratio in order to achieve PDMS films of the same thickness as for the less viscous SG184. Alternatively, lower thicknesses can also be achieved by increasing the spin-coating angular speed, but in our case we found that this resulted in an 
increased roughness of the SG186 substrate, therefore, we opted for the dilution with toluene. This process does not affect the viscoelastic properties of the final PDMS film, as toluene evaporates completely during spin-coating. This assumption has been verified by comparing our experimental results on SG186 prepared with and without dilution in toluene as shown in Appendix A.

Surface characterization of the prepared substrates by Atomic Force Microscopy (AFM) in Tapping Mode ${ }^{\mathrm{TM}}$ revealed an RMS roughness of $0.28 \pm 0.05 \mathrm{~nm}$.

In order to prepare substrates with elasticities that vary in-between those of pure SG184 and SG186, we prepare separately mixtures of SG184 and SG186 and, before degassing, we combine the two mixtures in the w/w ratios (SG184:SG186) 1:2, 1:3 and 1:6. Subsequently, we follow the same procedure as for the plain SG184 and SG186. Another method to reach intermediate elasticities, would be to mix the base and curing agent of e.g. SG184 with mixing ratios other than 10:1 in order to achieve different cross-linking ratios and thus different elasticities for the same type of PDMS. We did not follow this procedure, because the substrate produced would be a gel rather than a rubber. This would modify qualitatively the viscoelastic properties of the substrates produced with this method in comparison to the ones produced by full cross-linking (10:1 mixing ratio). Since the viscoelastic properties of our substrates play a very important role in the outcome and interpretation of our experimental work, we opt for the full cross-linking of mixtures of the two types of PDMS.

Dow Corning does not indicate the elastic moduli of SG184 and SG186, but it rather gives the hardness of the two types of PDMS in Durometer Shore A scale, which is a typical measure of hardness for polymers and it is shown in the left column of Table 2.1. In order to measure the elasticities of our substrates, we perform rheology measurements, whose results are given in the middle column of Table 2.1. As expected, the substrate with the highest elastic modulus is SG184. As this is mixed with SG186, its elastic modulus decreases until it reaches its lowest value for SG186.

In the third column of Table 2.1 we report the elastic moduli of our substrates measured on our experimental system with a technique we developed during the work on this thesis based on the Hertzian model. This technique and the exact experimental procedure will be presented more analytically in Chapter 3. Although the values extracted with the two techniques are in the same order of magnitude and present the same decreasing behaviour going from SG184 towards SG186, there is still a disagreement between the two techniques, which increases for the stiffer substrates. 


\begin{tabular}{|c|c|c|c|}
\hline PDMS type & $\begin{array}{c}\text { Durometer } \\
\text { Shore A }\end{array}$ & $\begin{array}{c}E \text { modulus [MPa] } \\
\text { (shear rheometer) }\end{array}$ & $\begin{array}{c}E \text { modulus [MPa] } \\
\text { (Hertzian model) }\end{array}$ \\
\hline SG184 & 50 & $2.7 \pm 0.4$ & $5.0 \pm 0.5$ \\
SG184(1)-SG186(2) & - & $2.3 \pm 0.5$ & $3.4 \pm 0.3$ \\
SG184(1)-SG186(3) & - & $2.1 \pm 0.5$ & $3.1 \pm 0.2$ \\
SG184(1)-SG186(6) & - & $1.7 \pm 0.2$ & $2.2 \pm 0.2$ \\
SG186 & 24 & $1.5 \pm 0.2$ & $1.5 \pm 0.1$ \\
\hline
\end{tabular}

Table 2.1: The elastic modulus of the different PDMS substrates as measured with the shear rheometer (middle column) and with the Hertzian model technique (right column). In the right column is reported the stiffness of SG184 and SG186 as given by Dow Corning.

The reasons for this can be summarised as follows: The elastic modulus of crosslinked PDMS depends, among others, on external factors, such as the curing procedure and the parameters used during the measurement itself (frequency, strain level etc) [48, 49]. The temperature control of the rheometer was achieved by circulation of hot water, therefore the maximum temperature attained was set at $80^{\circ} \mathrm{C}$. On the other hand, the temperature used to cure the PDMS films of our experimental system was $170^{\circ} \mathrm{C}$, i.e. much higher than the temperature at which the samples were annealed in order to induce the dewetting of the PS film $\left(120^{\circ} \mathrm{C}\right)$. In this way, any further crosslinking during the dewetting of the overlying film was avoided. Moreover, the actual measurement of the elastic modulus was done at $80^{\circ} \mathrm{C}$ for the rheology measurements (for the same reasons stated above), whereas for the PDMS films the temperature at which the elastic modulus was measured was the annealed temperature of the system in order to induce the dewetting of the PS thin film, i.e. $120^{\circ} \mathrm{C}$.

Furthermore, rheology measurements were performed in bulk, while our experimental system consisted of PDMS films. Elasticity is an extensive property of the system, i.e. it depends on the system size or the amount of material in the system, therefore, the final result in the two cases is expected to be different.

Finally, deviations in the geometry of the sample during the rheology measurements, such as the presence of air-bubbles in the bulk of the PDMS or the formation of menisci in its circumference in contact with the two plates of the rheometer, also contributed in the uncertainty of the results.

Despite the afore mentioned ambiguities, the results for the elastic moduli of our 
substrates from the rheology measurements proved to be very helpful at the beginning of our experiments as they gave an insight into our experimental system and were employed in a preliminary analysis of our results.

\section{Liquid substrates}

The liquid viscoelastic substrates used in our experiments are made of Poly-methylmethacrylate (PMMA), which is a glassy polymer, with glass transition temperature in bulk $T_{g} \sim 105^{\circ} \mathrm{C}$. An important physical property of PMMA that makes it suitable for our studies, is that, at the molecular weights and temperatures employed in our experiments, PMMA is immiscible with PS. PMMA with a monodispersity of $M_{w} / M_{n}=$ 1.04 was purchased from Polymer Standards Service (Mainz, Germany). In order to tune the viscosity, we use different molecular weights of PMMA as follows: $M_{w 1}=4.1 \mathrm{~kg} / \mathrm{mol}$, $M_{w 2}=10.1 \mathrm{~kg} / \mathrm{mol}, M_{w 3}=14.1 \mathrm{~kg} / \mathrm{mol}, M_{w 4}=20.7 \mathrm{~kg} / \mathrm{mol}, M_{w 5}=62.2 \mathrm{~kg} / \mathrm{mol}$, $M_{w 6}=89.3 \mathrm{~kg} / \mathrm{mol}, M_{w 7}=110.0 \mathrm{~kg} / \mathrm{mol}, M_{w 8}=520.0 \mathrm{~kg} / \mathrm{mol}$. The PMMA substrates are prepared by spin coating a toluene solution of PMMA directly onto a Si wafer with the proper spin-coating parameters in order to produce PMMA thin films of $180 \pm 5 \mathrm{~nm}$.

\section{Overlying thin liquid film}

The liquid used for dewetting in our experiments is atactic polystyrene (PS) with a monodispersity of $M_{w} / M_{n}=1.04$ and was purchased from Polymer Standards Service (Mainz, Germany). Polymers such as polystyrene (PS) are very suitable model liquids, since they have a very low vapour pressure in the melt and thus mass conservation is valid in our experiments. Moreover, they are chemically inert, non-polar and their dynamics can be tailored by choosing different chain lengths and annealing temperatures. The majority of the experiments was carried out using polystyrene with a molecular weight of $M_{w}=17.4 \mathrm{~kg} / \mathrm{mol}$, so that it could be assumed that we are dealing with a Newtonean liquid [50, 51], as already mentioned on page 24. Nevertheless, in cases where modification of polystyrene viscosity was necessary while keeping the temperature constant, different chain lengths were employed and this is mentioned in the text.

Although there exist several techniques for preparing thin polymer films, spin-coating is still a very convenient and efficient way to make ultra-thin, highly uniform polymer 
films in a non-equilibrium state on the substrate. Prior to spin-coating, PS was diluted in toluene in various concentrations depending on the desired thickness of the final PS film. Toluene is a good solvent both for PDMS and PMMA. Therefore, to avoid damaging the surface of the substrate, the PS-toluene solution cannot be spin-coated directly on the substrate. The option of using another solvent instead of toluene was also taken into consideration. For example cyclohexane is a good solvent for PS (and PDMS) and a poor one for PMMA. Nevertheless, no good solvent for PS was available that was at the same time a poor solvent for PDMS. Therefore, the thin polystyrene films were prepared by spin-coating PS onto a freshly cleaved mica sheet. The solidified PS film was floated on Millipore ${ }^{\mathrm{TM}}$ water and transferred onto the previously prepared viscoelastic substrate. Atomic force microscopy (AFM) measurements in Tapping Mode ${ }^{\mathrm{TM}}$ revealed the equilibrium contact angle of PS on PDMS to be $\theta_{e, P D M S}=62 \pm 1^{\circ}$, whereas the equilibrium contact angle on PMMA is $\theta_{e, P M M A}=7 \pm 2^{\circ}$.

In order to observe dewetting, the polymer thin film has to be transformed by a rupture mechanism from a spatially homogeneous to a spatially inhomogeneous layer. Necessary precondition to that is that the film be either metastable or unstable on the substrate. Based on the theory given in Section 1.1, the van der Waals potential of an experimental system can be readily determined, provided the Hamaker constant can be calculated by the optical properties of the materials involved and the thickness of the PDMS layer is known. Thus, to determine the effective interface potential and thus the stability of our two systems, we first calculate the Hamaker constants, which were found to be: $A_{\text {air } / P S / P D M S}=2.69 \cdot 10^{-20} \mathrm{~J}$ for our two-interface-system of air/PS/PDMS (the PDMS layer is thick enough to neglect the effect of the underlying $\mathrm{SiO}_{2} / \mathrm{Si}$ layers) $A_{\text {air } / P S / P M M A}=1.33 \cdot 10^{-20} \mathrm{~J}$ and for the system air/PS/PMMA.

From the shape of the curves given in Figure 2.2, one could assume that the dewetting pattern that we observe corresponds to spinodal dewetting. Nevertheless, as we will see later in Figure 4.3. (a) the holes are randomly (Poisson) distributed, thus they are assumed to stem from heterogeneous nucleation, as nuclei typically exhibit random statistics. Actually, the experimental distinction between spinodal dewetting and heterogeneous nucleation is far from being obvious: the thicker the film, the smaller the driving force and hence, the larger the growth time $\tau$ of the spinodal mode (typically $\left.\tau \propto h^{5}\right)$. Therefore, for thicker films, dewetting by heterogeneous nucleation may be quicker and can suppress a spinodal pattern [52], which is actually the case for our experimental system, where the film thicknesses range from $50 \mathrm{~nm}$ to $200 \mathrm{~nm}$, significantly 


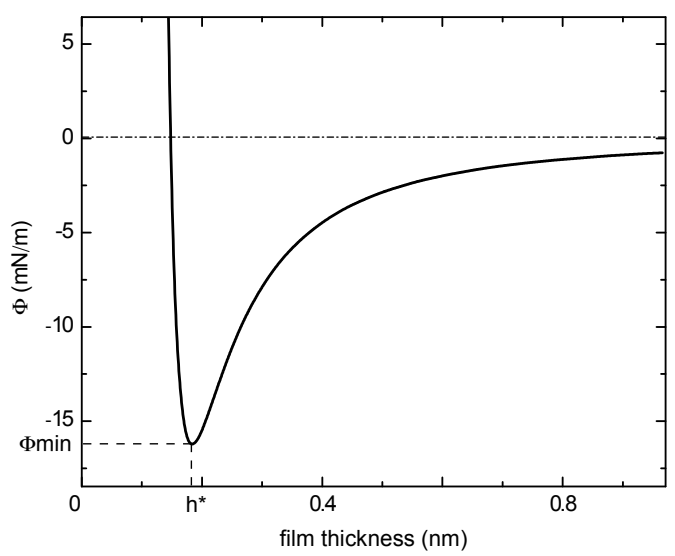

(a)

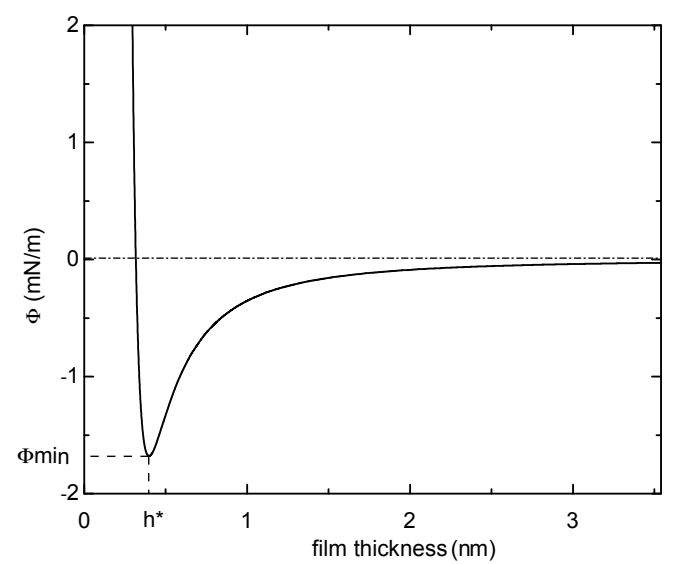

(b)

Figure 2.2: Effective interface potential $\Phi$ as a function of film thickness $h$ for the system (a) air/PS/PDMS and (b) air/PS/PMMA.

higher than the equilibrium film thickness $h_{\text {air } / P S / P D M S}^{*} \sim 0.18 \mathrm{~nm}$ (Figure 2.2.(a)) and $h_{\text {air } / P S / P M M A}^{*} \sim 0.39 \mathrm{~nm}$ (Figure 2.2.(b)).

\subsubsection{Topographically structured viscoelastic substrates}

The preparation of the topographically structured PDMS substrates is done by using a topographically structured Si wafer as a template onto which the PDMS is molded. The Si templates were purchased from IMS-Chips (Stuttgart). On one single Si wafer of $15 \mathrm{~cm}$ diameter, there are several areas of rectangular grooves with $30 \mu-m 40 \mu m$ depth, aspect ratios of width to depth that range from $1: 3$ to $1: 10$ and various wall thicknesses and groove widths (Figure 2.3.

In order to be able to use the Si wafer as a master, we need to be able to easily peel off the PDMS from the wafer after curing. To do so, it is substantial that we lower the surface energy of the Si wafer by coating it with a self-assembled monolayer (SAM). The cleaniness of the Si wafer plays an important role in order to achieve a high quality SAM. Initially, we follow the cleaning procedure that has been described in Section 2.1.1 on page 40. Subsequently, the samples are kept in Piranha Solution $\left(50 \% \mathrm{H}_{2} \mathrm{SO}_{4}\right.$ and $50 \% \mathrm{H}_{2} \mathrm{O}_{2}$ ) for $1 \sim 2$ hours and they are then flushed thoroughly with hot Millipore ${ }^{\mathrm{TM}}$ water in order to remove any traces of piranha. 


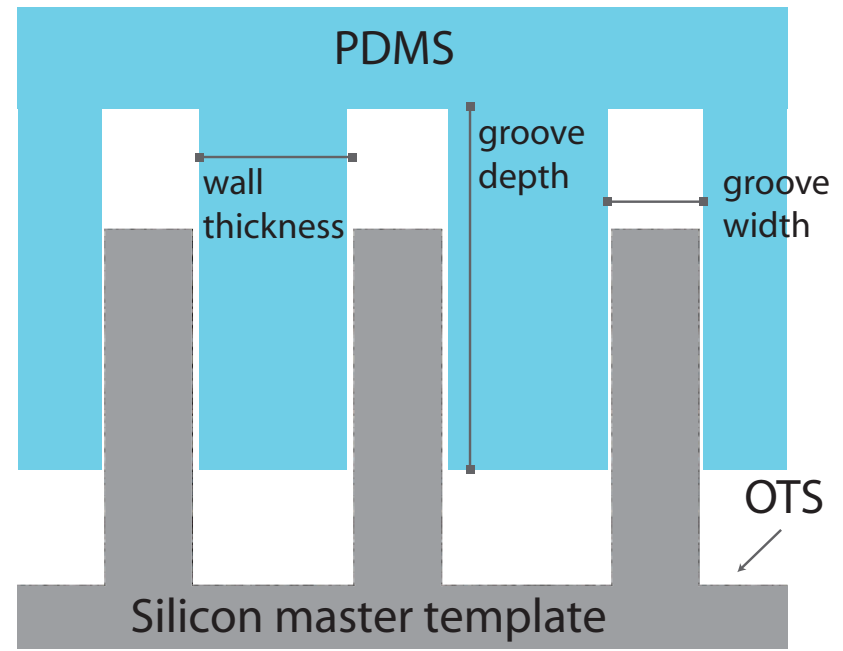

Figure 2.3: Diagram illustrating the fabrication of a PDMS stamp.

The molecule chosen for the Si wafer coating is Octadecyltrichlorosilane (OTS) (Figure 2.4), because, compared to other SAMs, it gives a very good surface quality, as it has a very small contact angle hysteresis and very low roughness [53].

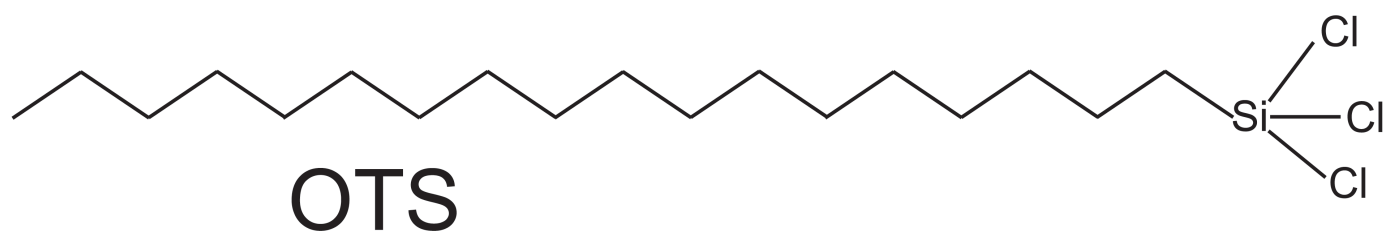

Figure 2.4: Schematic representation of an Octadecyltrichlorosilane (OTS) molecule.

To deposit OTS, we immerse the cleaned substrates in a bath [54] of:

- $50 \mathrm{ml}$ of Bicyclohexane

- 30 drops of Carbontetrachloride

- 15 drops of OTS

for $3 \sim 4$ hours. During this time, the $-\mathrm{SiCl}_{3}$ head groups of the OTS molecules form covalent bonds with the hydroxyl groups of the Si wafer, while the hydrophobic tail of 
alkyl groups assemble far from the substrate, creating thus a hydrophobic surface. In order to avoid the formation of "islands" of OTS on the Si wafers that would lead to a less ordered OTS layer, in the first 15 minutes that the wafers are in the solution, we regularly take them out of the solution and rinse them with chloroform, eliminating thus the excess OTS. At the end, the samples are removed from the solution, rinsed again with chloroform and dried carefully.

In the meantime, we have prepared a mixture of PDMS as described on page 41, and we pour it on the Si "master". We degas again for 5 minutes in order to break any gas bubbles that might have formed while pouring the PDMS on the Si wafer and we cure it in a protected atmosphere $\left(N_{2}\right)$ at $170^{\circ} \mathrm{C}$ for one hour. Subsequently, we carefully remove the PDMS from the Si wafer and we end up with a viscoelastic substrate that has a pattern that mirrors the Si master (Figure 2.3).

\section{$2.2 \quad$ Experimental techniques}

\subsubsection{Optical microscopy}

\section{Widefield optical microscopy}

Light microscopy is an important investigative tool in many scientific sectors. During this thesis work, a Leica microscope was used in brightfield reflection mode in combination with a Lincam heating stage, in order to image in situ and capture the dewetting process of our PS film with a CCD camera. Images were then analysed with the software "Image Pro Plus 5.0" in order to extract the radius over time behaviour of the single holes.

Standard reflection brightfield microscopy relies upon light from the light source being gathered by a condenser lens and shaped into a cone whose apex is focused at the plane of the specimen. Specimens are seen because of their ability to change the speed and the path of the light reflected on them. This ability is dependent upon the refractive index and the opacity of the specimen. If the specimen has a refractive index 


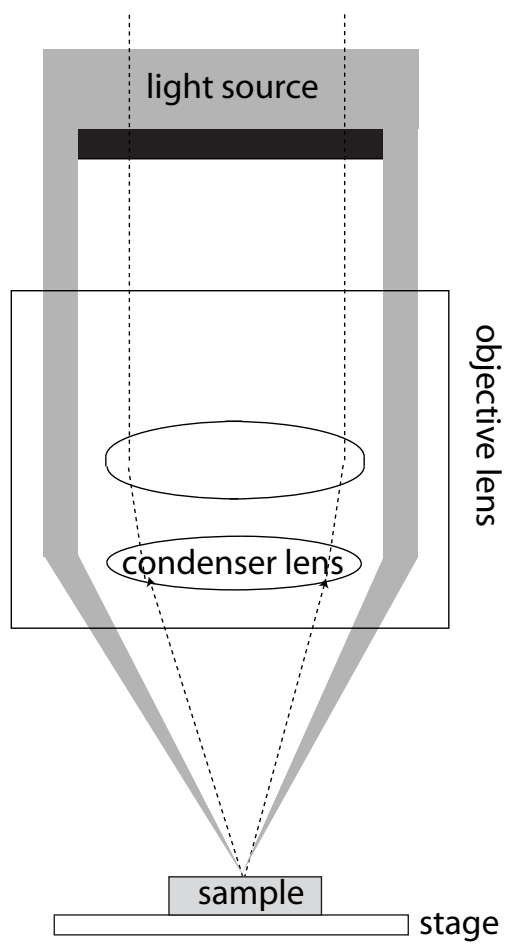

Figure 2.5: Diagram illustrating the light path through a dark field microscope.

too similar to the surrounding medium between the microscope stage and objective lens, it will not be seen and darkfield microscopy has to be employed.

Darkfield microscopy relies on a different illumination system from that of brightfield microscopy. Rather than illuminating the sample with a filled cone of light, the condenser is designed to form a hollow cone of light. As shown in Figure 2.5, the light at the apex of the cone is focused at the plane of the specimen. As this is reflected from the specimen plane, it spreads again into a hollow cone. When a sample is on the stage, the light at the apex of the cone strikes it. The image is made only by those rays scattered by the sample and captured in the objective lens and it appears bright against the dark background. This situation can be compared to the glittery apearance of dust particles in a dark room illuminated by strong shafts of light rays.

For the scope of this thesis, a Mitutoyo microscope in darkfield mode was used in order to check the mechanical cleanliness, i.e. the amount of remaining particles, of the viscoelastic substrate before the PS film was transferred onto it. 


\section{Confocal Microscopy}

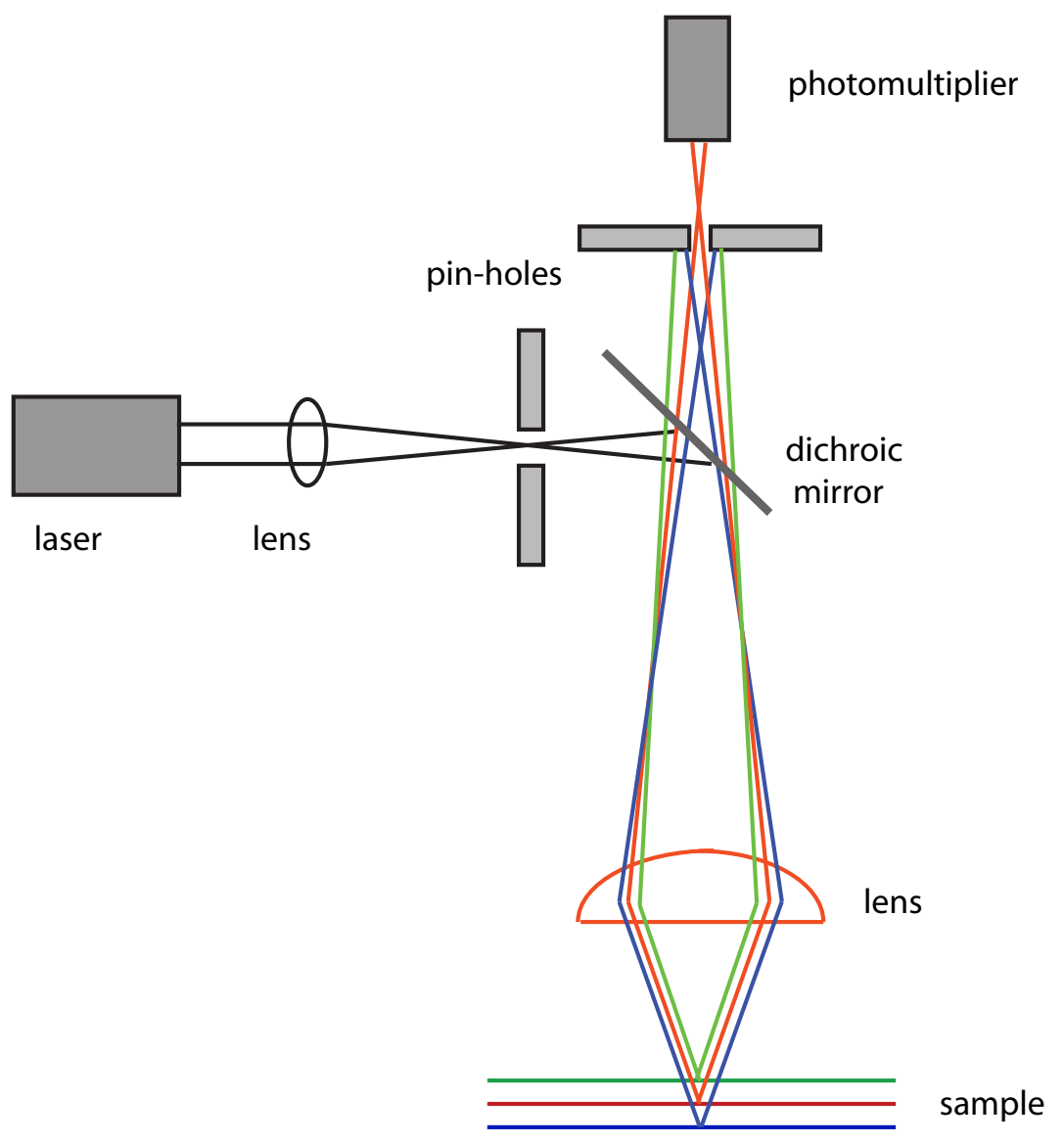

Figure 2.6: Schematic representation of a confocal microscope.

A Leica confocal microscope was used during this experimental work in order to determine the thickness of the plain PDMS substrates and to characterise geometrically the topographically structured PDMS substrates.

Confocal microscopy offers several advantages over conventional widefield optical microscopy, including the ability to control depth of field, elimination or reduction of background information away from the focal plane (that leads to image degradation) and the capability to collect serial optical sections from thick specimens. The basic key to the confocal approach is the use of spatial filtering techniques to eliminate out-of-focus 
light or glare in specimens whose thickness exceeds the immediate plane of focus.

The confocal principle in epi-fluorescence laser scanning microscopy is diagrammatically presented in Figure 2.6. Coherent light emitted by the laser system (excitation source) passes through a pinhole aperture that is situated in a conjugate plane (confocal) with a scanning point on the specimen and a second pinhole aperture positioned in front of the detector (a photomultiplier tube). As the laser is reflected by a dichromatic mirror and scanned across the specimen in a defined focal plane, secondary fluorescence emitted from points on the specimen (in the same focal plane) passes back through the dichromatic mirror and is focused as a confocal point at the detector pinhole aperture.

The significant amount of fluorescence emission that occurs at points above and below the objective focal plane is not confocal with the pinhole and forms extended Airy disks in the aperture plane. Because only a small fraction of the out-of-focus fluorescence emission is delivered through the pinhole aperture, most of this extraneous light is not detected by the photomultiplier and does not contribute to the resulting image. The dichromatic mirror, barrier filter, and excitation filter perform similar functions to identical components in a widefield epi-fluorescence microscope. Refocusing the objective in a confocal microscope shifts the excitation and emission points on a specimen to a new plane that becomes confocal with the pinhole apertures of the light source and detector.

\subsubsection{Atomic Force Microscopy (AFM)}

Atomic Force Microscopy has been an invaluable investigative tool for the realization of the experimental work for this thesis. It gave insight regarding the contact angles of the dewetting liquid on the different substrates, the surface topographies and the material properties of our system. In the following section, the basic features and characteristics of an Atomic Force Microscope are presented.

The basic implementation of an AFM is sketched in Figure 2.7. The AFM consists of a microscale cantilever with a sharp tip (probe) at its end that is used to scan the specimen surface. When the tip is brought into proximity with a sample surface, forces between the tip and the sample lead to a deflection of the cantilever according to Hooke's law. The deflection is measured using a laser spot, reflected from the top surface of the cantilever into an array of position-sensitive photodiodes. 


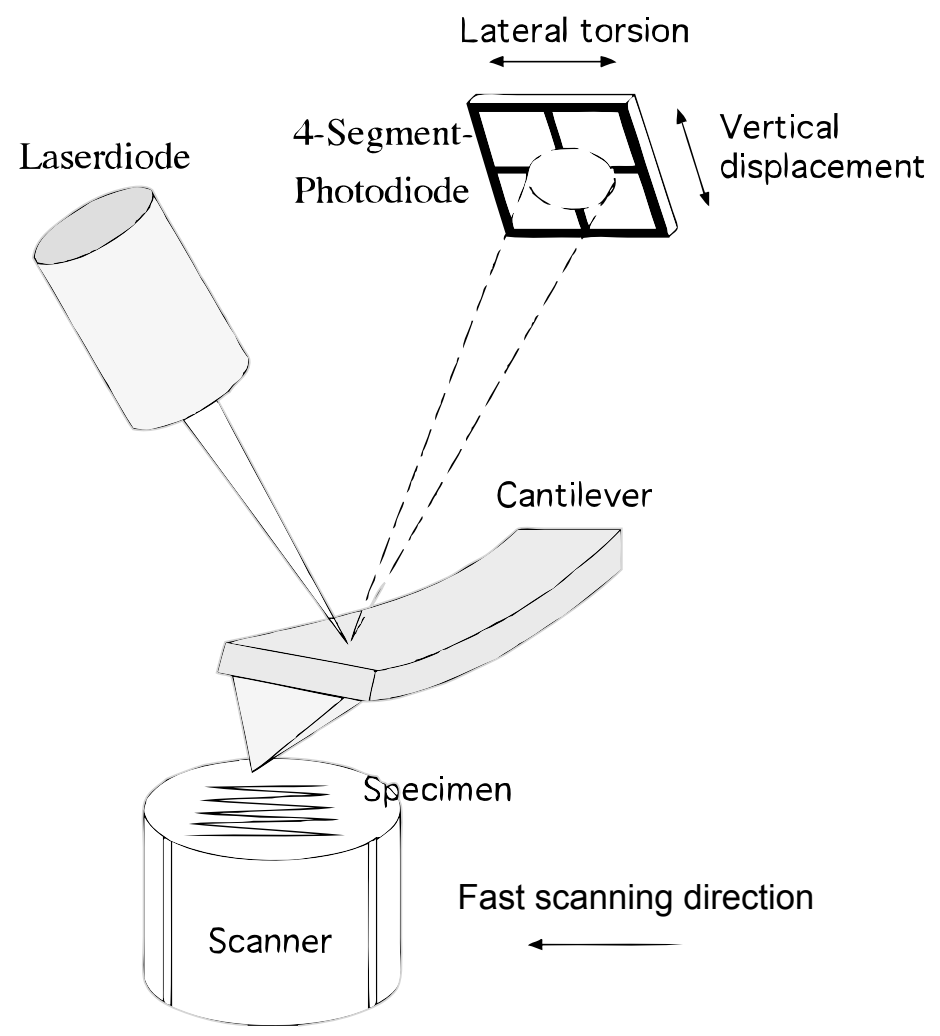

Figure 2.7: General principle of an Atomic Force Microscope. Force acting on the probe tip causes the cantilever to bend, action that is monitored by a sensor. The signal is then used in the scanner feedback, which regulates the sample-tip interaction.

The force experienced by a probe approaching a surface is illustrated in Figure 2.8. Initially there is a weak repulsive force until the probe "jumps" to contact with the surface layer. Further movement towards the surface results in bending of the cantilver due to the stiffness of the surface and the probe remains stuck to the surface until it can overcome the adhesive forces between the tip and the sample. The force applied after the tip has made contact with the surface can provide a measurement of the stiffness or compliance of the sample, while the force required to pull the sample up from the surface is a measure of the adhesion between the tip and the sample. 


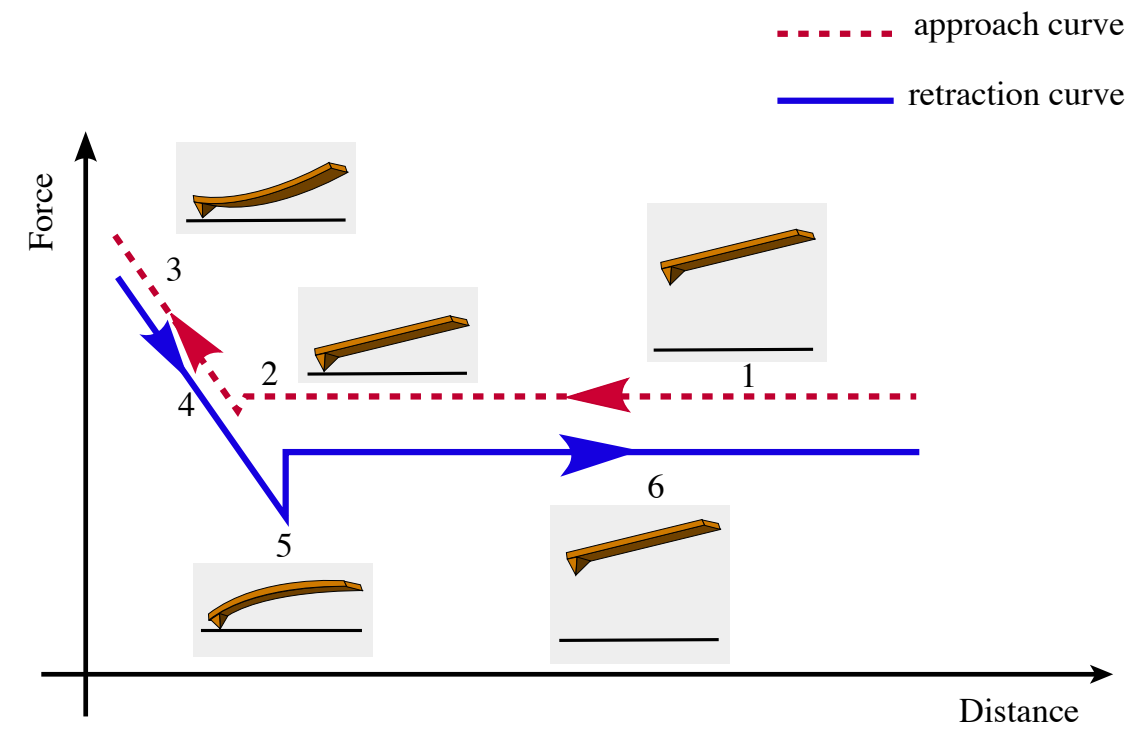

Figure 2.8: Force-distance curve describing a single approach-retract cycle of the AFM tip. The AFM tip is approaching the sample surface (1). The initial contact between the tip and the surface is mediated by the attractive van der Waals forces (contact) that lead to an attraction of the tip towards the surface (2). Hence, the tip applies a constant and default force upon the surface that leads to sample indentation and cantilever deflection (3). Subsequently, the tip tries to retract and to break loose from the surface (4). Various adhesive forces between the sample and the AFM tip, however, hamper the tip retraction. These adhesive forces can be taken directly from the forcedistance curve (5). The tip withdraws and loses contact to the surface upon overcoming the adhesive forces (6).

\section{Interaction forces and imaging modes}

Depending on the situation, the forces between the tip and the sample that lead to a deflection of the cantilever include mechanical contact forces, Van der Waals forces, capillary forces, chemical bonding, electrostatic forces and magnetic forces. In order to determine which measurement mode to employ, a rough idea of the forces involved is needed. From a physical point of view, one can make a distinction between different operating modes depending on the sign of the forces in the interaction between the tip and the sample (Figure 2.9). In general, possible imaging modes are divided into static modes and a variety of dynamic modes where the cantilever is vibrated. 


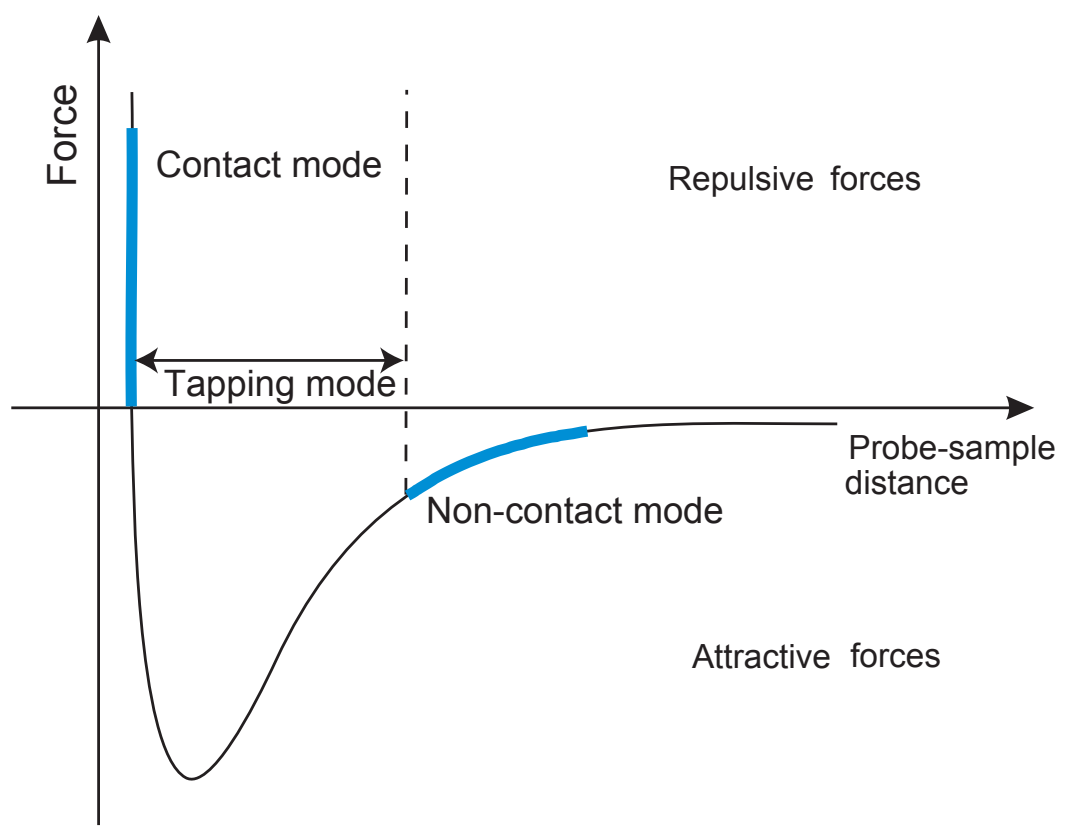

Figure 2.9: Sketch of the forces between the tip and the sample, highlighting where typical imaging modes are operative.

Contact mode: The contact mode is the direct AFM mode in which the tip is brought in hard contact with the surface. In this mode of operation, the static tip deflection is used as a feedback signal. During the scan, the cantilever deflection is kept constant by the feedback loop. Because the measurement of a static signal is prone to noise and drift, low stiffness cantilevers are used to boost the deflection signal. However, close to the surface of the sample, attractive forces can be quite strong, causing the tip to "snap-in" to the surface. Thus the static mode AFM is always done in regime where the overall force is repulsive. Since the tip is permanently in contact with the surface while scanning, a considerable shear force is generated, which might cause damage to the surface. For contact mode imaging, cantilevers made from SiN are generally used because they have low stiffness in the rage of $0.06 \mathrm{~N} / \mathrm{m}$ to $1 \mathrm{~N} / \mathrm{m}$. Image contrast depends on the applied force, which depends on the cantilever spring constant.

Non-contact mode: The non-contact mode is implemented in situations where tip contact might alter the sample in subtle ways. In this mode, the probe operates in the attractive force region and the tip-sample interaction is minimized. The tip of the cantilever does not contact the sample surface. Instead, during scanning, it hovers 
$50-150 \AA$ above the sample surface. The forces used to construct the input signal are the attractive Van der Waals forces, which are substantially weaker than the forces used by contact mode. Therefore, the tip must be given a small oscillation, so that a change in amplitude, phase or frequency of the oscillating cantilever can be detected as a response to force gradients from the sample. With the constant aid of feedback, the system moves the scanner up and down at each $(\mathrm{x}, \mathrm{y})$ data point in order to keep the resonant frequency and amplitude constant. The average tip-to-sample distance is therefore kept constant as well. It is this motion of the scanner that is used to generate the data sheet stored to form the topographic image of the sample surface.

Tapping $^{\text {TM }}$ mode: In Tapping ${ }^{\mathrm{TM}}$ mode the cantilever is driven to oscillate up and down at near its resonance frequency by a small piezoelectric element mounted in the AFM tip holder. The amplitude of this oscillation is typically greater than $20 \mathrm{~nm}$. The interaction of forces acting on the cantilever when the tip comes close to the surface, Van der Waals forces or dipole-dipole interaction, electrostatic forces, etc., causes the amplitude of this oscillation to decrease as the tip gets closer to the sample. As in non-contact mode, the feedback loop adjusts the height to maintain a set cantilever oscillation amplitude as the cantilever is scanned over the sample. Tapping ${ }^{\text {TM }}$ mode cantilevers: OMCL160TS Olympus Cantilevers (Japan) were typically used for the Tapping $^{\mathrm{TM}}$ mode imaging. The probes mounted on those cantilevers are tetrahedral in shape and asymmetric from the side view. The front and back sides of the tip are inclined at $0^{\circ}$ and $35^{\circ}$ respectively from the cantilever vertical axis. Furthermore, the

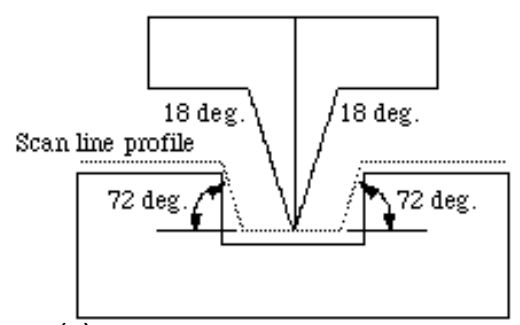

(a)

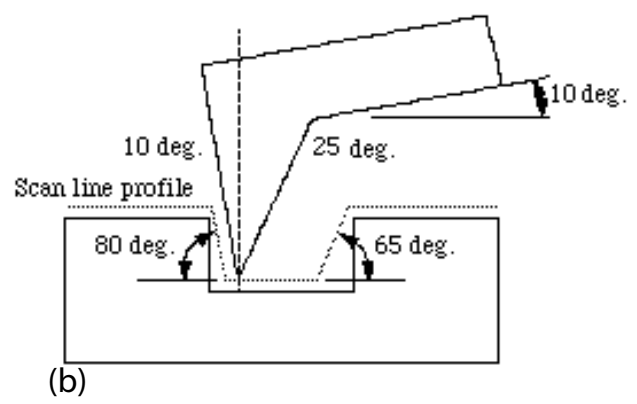

Figure 2.10: (a) Front side tip profile, (b) Lateral tip profile.

cantilever is mounted on the cantilever holder at an inclination angle of $10^{\circ}$, resulting in the maximum detectable slope being $80^{\circ}$ on the "left side" and $65^{\circ}$ on the "right side" respectively (Figure 2.10), which is a feature that has to be considered when imaging 
sharp topographies such as the ones we are dealing with.

\section{Harmonix $^{\mathrm{TM}}$}

As described above, in Tapping ${ }^{\mathrm{TM}}$ mode AFM, the cantilever is excited and vibrates at its resonant frequency as it is brought close to the sample. Intermittent contact with the surface alters the amplitude and phase of the cantilever vibration, which are the two primary observables and can be approximated by a sinusoidal trajectory. Nevertheless, these quantities provide only time-averaged values of the tip-sample interaction forces [55]. Therefore, the information about how the interaction force varies as the tip undergoes its trajectory is lost to a large extent. Such information can be given by the use of higher harmonic vibrations excited by the tip-sample forces [56 61].

There is, however, a major difficulty in this approach: the overall frequency response of the cantilever, required for translating harmonic vibration signals into harmonic forces acting on the tip, depends on the shape of the cantilever mode and the position of the laser spot [62]. It is difficult to determine all of these parameters accurately. As a solution, the Harmonix ${ }^{\mathrm{TM}}$ cantilever (Figure 2.11), having a tip that is offset from

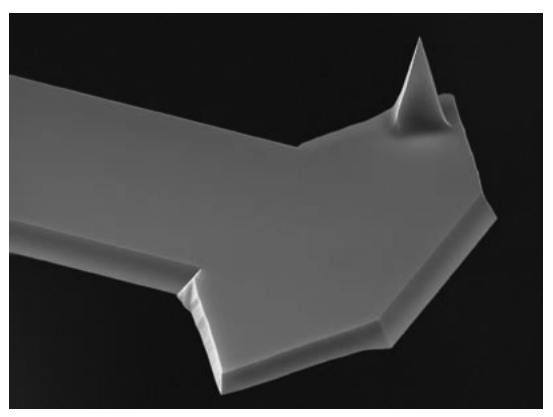

Figure 2.11: SEM image of a Harmonix ${ }^{\mathrm{TM}}$ cantilever used to quantitatively map in real-time elastic and dissipative forces during Tapping ${ }^{\mathrm{TM}}$ mode imaging.

the long axis of the cantilever, enhances the signals at higher harmonics and provides frequency response that can be accurately determined. When it is vibrated in the tapping mode, tip-sample interaction forces generate a torque around the long axis of the cantilever and excite the torsional modes.

This method has the inherent nanometre scale lateral resolution of the Tapping ${ }^{\mathrm{TM}}$ 
mode AFM and, compared to conventional tools, it exhibits significant improvement in temporal and spatial resolution as well as in indentation and mechanical loading.

Throughout the majority of the experiments, a Dimension V AFM (Veeco, Santa Barbara, USA) has been used. In the case of roughness measurements, where a very high signal to noise ratio is required and in the case of online measurements at elevated temperatures with a heating stage, a Multi Mode V AFM (Veeco, Santa Barbara, USA) has been employed. All of the experiments were performed in Tapping ${ }^{\mathrm{TM}}$ mode operation. In parallel, for a set of experiments where the real-time investigation of the material properties was necessary, a Dimension Icon AFM (Veeco, Santa Barbara, USA) in Harmonix ${ }^{\mathrm{TM}}$ - Tapping ${ }^{\mathrm{TM}}$ mode was used.

\subsubsection{Rheometry}

Rheometry has been employed in order to measure the elastic modulus of our rubber substrates and confront it with the results of other independent experimental techniques. Rheological measurements were carried out using a Haake Mars Rheometer from Thermo Scientific Karlsruhe that was operated in temperatures up to $80^{\circ}$, kindly provided by Prof. C. Wagner, at the Department of Technical Physics of the University of Saarland.

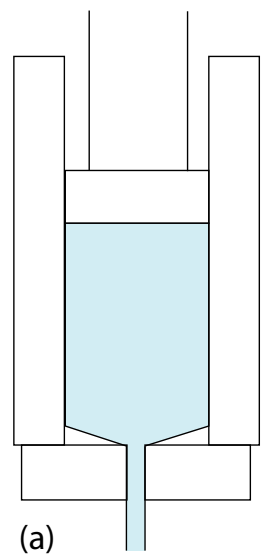

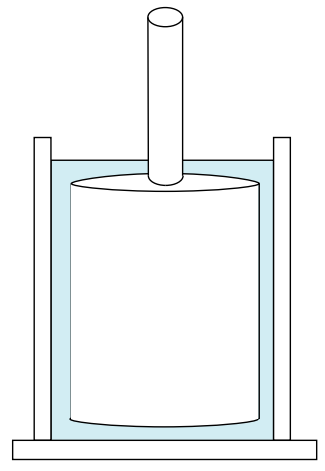

(b)

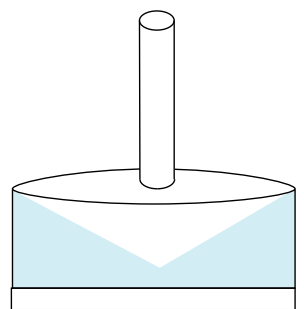

(c)

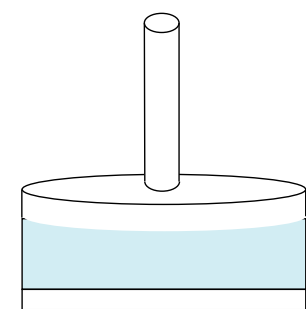

(d)

Figure 2.12: Sketch of shear rheometers of different geometries: (a) capillary (b) rotational cylinder, (c) cone-plate, (d) plate-plate. 
There are two distinctively different types of rheometers, depending on the geometry of the applied stress. When the applied stress is tensional, the rheometer is called extensional rheometer, whereas, when shear stress is applied, the rheometer is called shear rheometer. In this section, we will deal with the latter.

As seen in Figure 2.12, the shear rheometers can be categorised into three main groups:

- Capillary, in which the liquid is forced through a tube of constant cross-section and precisely known dimensions under conditions of laminar flow (Figure 2.12.(a)). Either the flow-rate or the pressure drop is fixed and the other is measured. Knowing the dimensions, the flow-rate can be converted into a value for the shear rate and the pressure drop into a value for the shear stress. Varying the pressure or flow allows for a flow curve to be determined. The capillary rheometer is a common device for measuring viscosities.

- Rotational cylinder, in which the liquid is placed within the annulus formed by one cylinder being inside the other (Figure 2.12.(b)). One of the cylinders is rotated at a set speed. This determines the shear rate inside the annulus. The liquid tends to drag the other cylinder around, and the force it exerts on that cylinder (torque) is measured and converted to a shear stress. This type of rheometer is used widely for determining the flow character of drilling fluids.

- Cone and plate, The cone and plate geometry consists of an inverted cone in near contact with a lower plate (Figure 2.12. (c)). The cone is usually designed with an angle of less than $4^{\circ}$. Either the upper or lower surface may rotate depending on the instrument design. The parallel plate geometry can be considered a simplified version of the cone and plate, having an angle of $0^{\circ}$ (Figure 2.12.(d)). The test fluid is constrained in the narrow gap between the two surfaces. Cone and plate measurement tools are most often used for highly viscous pastes, gels, and concentrated suspensions.

Given the rheological properties of the PDMS before cross-linking, a shear rheometer in plate - plate geometry was chosen for our measurements. PDMS was prepared as explained on page 41 and was cured in situ, while the change of its rheological properties during curing was monitored and measured. 


\section{Chapter 3}

\section{Polystyrene nanodroplets on rubber elastic substrates}

In this chapter we analyse the complete shape of PS nanodroplets in equilibrium on rubber elastic substrates. Even though nanodroplets in equilibrium is the last stage of dewetting, we start our analysis from here, because it will give us information that is necessary in order to analyse and comprehend the more complicated phenomena involved in the dewetting dynamics, which we will discuss in Chapter 4.

\subsection{From rigid to deformable substrates}

When a liquid nanodroplet is deposited on a solid surface, gravity effects can be neglected and its shape can be approximated to a spherical cap. The equilibrium contact angle $\theta_{e}$ of the droplet with the substrate is a result of the balance between the solid/liquid, solid/vapour and liquid/vapour interfacial free energies. This balance is represented by Young's equation [17]

$$
\cos \theta_{e}=\frac{\gamma_{S V}-\gamma_{S L}}{\gamma_{L V}}
$$

Equation 3.1 was derived considering a perfectly smooth, homogeneous and rigid solid surface. If now the droplet is placed on an elastic surface, it causes a deformation, whose 
shape depends on the properties of the materials involved and their surface interactions. Specifically, the sessile drop pulls upwards the surface at the three-phase contact line (TPCL), while it pushes downwards at its contact area due to the Laplace pressure inside the liquid (Figure 3.1).

Pericet-Càmara et al. [63] were the first ones to image experimentally in-situ the effect of capillary pressure on the deformation of an elastic surface caused by a sessile microdrop. Their experiments involved imaging by laser scanning confocal microscopy the deformation of a silicone elastomeric polymer below a microdrop of an ionic liquid, as well as the rim of the external profile of the deformation in the vicinity of the TPCL using a white-light confocal profilometer. The resolution of the techniques used, as the authors sustain, was not high enough for the specific system, such that no precise and detailed imaging could be achieved, but, even so, there was a satisfactory agreement between their experimental results and the theoretical curves extrapolated from the Linear Elasticity Theory for the specific system.

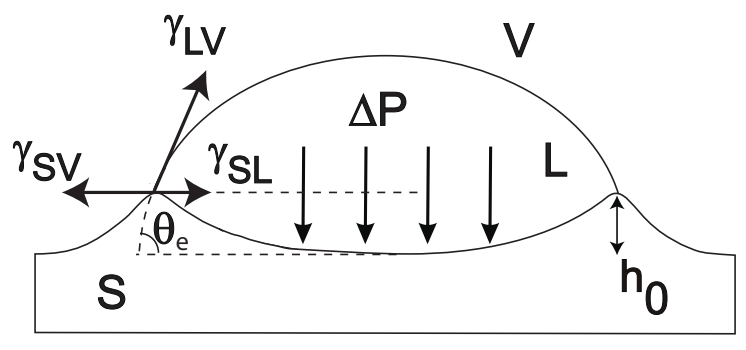

Figure 3.1: Sketch of a droplet on a viscoelastic substrate. The dotted line represents the solid without the deformation caused by the vertical component of the liquid surface tension, $\gamma_{L V} \cdot \sin \theta_{e}$. The system has not been sketched to scale, as the wetting ridge near the three-phase contact line of the solid (S)/liquid (L)/vapour (V) phases has been emphasized in order to allow for a better viewing.

In the following paragraphs, we analyse the shape of nanometre-sized polystyrene droplets generated by the complete dewetting of PS films on PDMS substrates of different elasticities, taking into account both the air/PS interface and the PS/PDMS interface. Our interest is focused mainly on the shape of the deformation at the outer part of the droplet in the vicinity of the three-phase contact line, as well as on the shape of the deformed substrate below the droplet. 


\subsection{Imaging the deformed PS/PDMS interface}

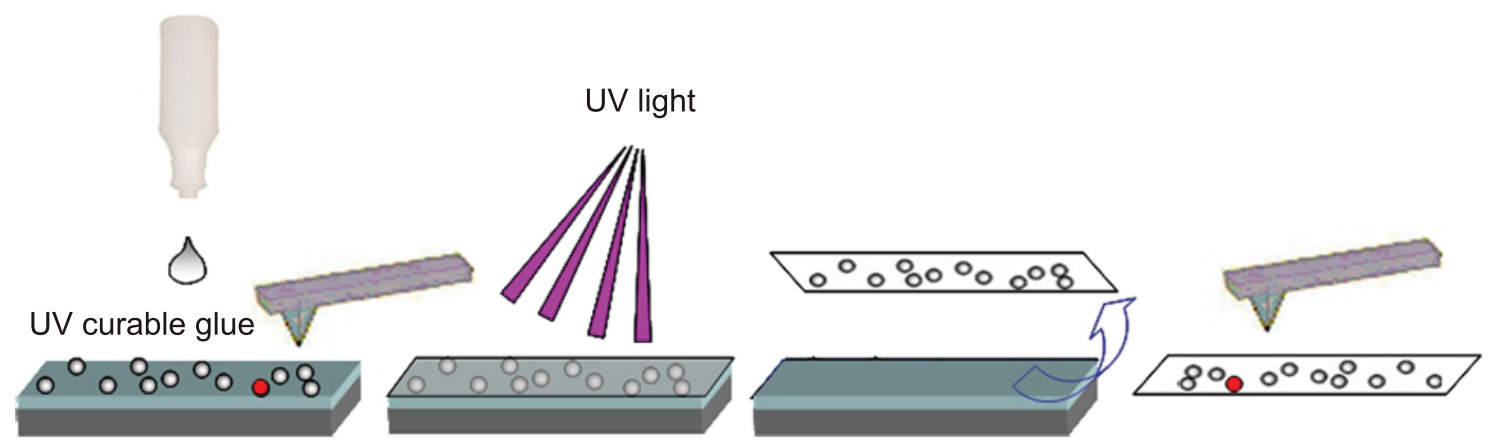

Figure 3.2: Schematic representation of the lift-off technique.

How does this deformed interface look like and how can we image it? Since PDMS is a rubber, it restores its initial shape when the PS film is removed, therefore, we cannot image the deformation directly on the substrate. To overcome this obstacle, we have developed a technique, where we lift-off the partially or totally dewetted PS film in its glassy state and image its "bottom side", which corresponds to the shape of the PS/PDMS interface. To do that, we identify the specific feature we are interested in and we first image by AFM its air/PS interface (Figure 3.3.(a)). Consequently, we pour a drop of UV curable glue (NOA 60, Edmund Optics, Karlsruhe, Germany) 64] on the sample and induce its spreading by covering it with a microscopy slide. After the glue has been cured by UV light exposure $(\lambda=366 \mathrm{~nm})$ for $15 \mathrm{~min}$, we lift off the microscopy slide and concurrently remove with it the solidified UV curable glue and the polystyrene morphologies partly "immersed" in the glue (Figure 3.2). Using this protocol, we end up with a glass support covered with a film of cross-linked polymer glue on which our polystyrene features are placed with their previously "bottom side" (liquid/PDMS interface) now facing upwards. We identify the particular polystyrene feature on which we had earlier performed the AFM measurement and image it again (Figure 3.3.(b)). This image of the air/PS interface corresponds to the former PS/PDMS interface, into which the substrate deformation is "frozen". As one can see, the stress that this nanodroplet applies on the substrate generates a characteristic craterlike shape 

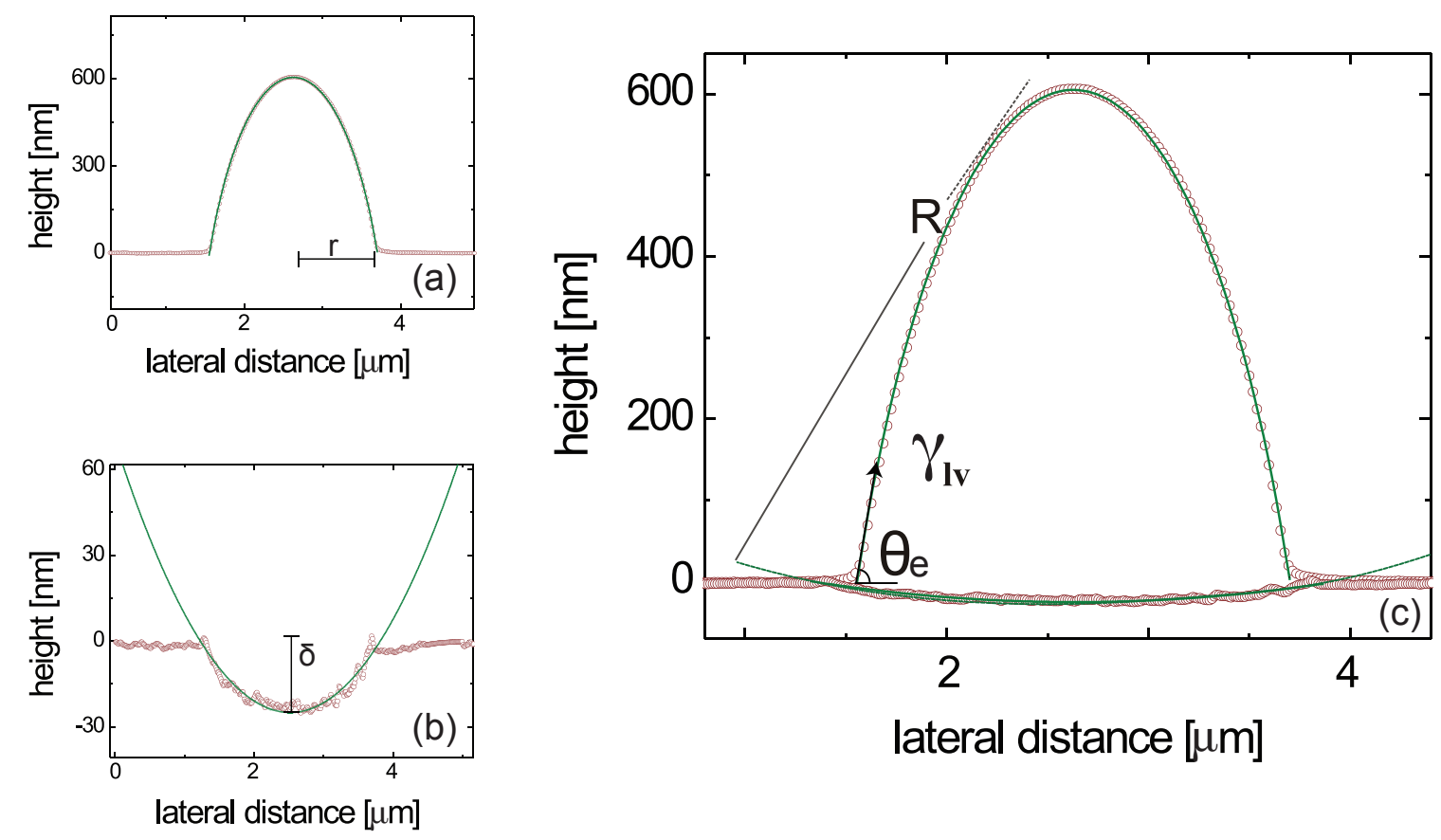

Figure 3.3: (a) Cross-section of an AFM scan of the air/PS interface of a sessile droplet of radius $r$ on PDMS (open circles) and the fit to a circular arc (green solid line). (b) Cross-section of an AFM scan corresponding to the PS/PDMS interface of a sessile droplet causing an indentation depth $\delta$ on the substrate (open circles) and the fit to a circle (green solid line). (c) Combination of the cross-sections (a) and (b) showing the complete droplet profile.

with indentation depth $\delta$. Both the air/PS and the PS/PDMS interface of the droplet can be fitted to spherical caps. By combining the two scans of the top Figure 3.3. (a) and bottom Figure 3.3. (b) side of the droplet, we manage to "reconstruct" the complete $3 \mathrm{~d}$ shape of the polystyrene droplet of radius $r$, deposited on the PDMS substrate and deforming it (Figure 3.3. (c)). 


\subsection{Extraction of the substrate elastic modulus for small droplets}

The reconstruction of the full shape of the droplet can also be used for the calculation of the elastic modulus of the substrate, by approximating the system PS/PDMS to a continuum model [65] that, given the contact area, can predict the elastic modulus for different geometries. In fact, we can consider the droplet to be a rigid indenter of radius $R$, which corresponds to the radius of the circular arc fitted to the bottom side of the droplet in Figure 3.3. (c). Additionally the rubber substrate is approximated by an elastic semi-infinite plane and the total force between them is given by the force that the vertical component of the surface tension is applying on the substrate along the perimeter of the droplet:

$$
F=2 \pi r \gamma_{L V} \sin \theta_{e}
$$

where $\gamma_{L V}$ is the surface tension of the system air/PS, $\theta_{e}$ is the contact angle of PS on PDMS and $r$ is the radius of the contact area of the PS droplet on the PDMS. With these approximations we can accurately describe our system with the Hertzian model [66] that correlates the radius of the circle of contact $r$ to the force $F$, the spherical indenter radius $R$ and the elastic properties of the deformable material:

$$
r^{3}=\frac{3 F R\left(1-\nu^{2}\right)}{4 E}
$$

where $E$ and $\nu$ are respectively the elastic modulus and Poisson's ratio of the elastic plane. We consider the PDMS rubber to be incompressible, thus $\nu=0.5$. Resolving Equation 3.3 for $E$, we find that $E$ is given by:

$$
E=\frac{3}{4}\left(1-\nu^{2}\right) F R r^{-3}
$$

Applying some simple trigonometry, we find that $R=\left(r^{2}+\delta^{2}\right) / 2 \delta$ and since in our case $r>>$, we can safely write $R \sim r^{2} /(2 \delta)$. Substituting $R$ and $F$ in Equation 3.4 , we find:

$$
\delta=\frac{3}{4}\left(1-\nu^{2}\right) \pi \gamma_{L V} \sin \theta_{e} E^{-1}
$$

which returns the indentation depth $\delta$ as a function of substrate elasticity $E$. We can 
observe that, with the approximation that we made, i.e. $r>>\delta$, thus for sufficiently small drops, the indentation depth does not depend on the drop radius. Quantifying the deformation that droplets cause to the substrate and using Equation 3.5, we can extrapolate the elastic modulus for each of our substrates. Our results based on this technique are given in Table 3.1 .

\begin{tabular}{|c|c|}
\hline PDMS substrate & $E$ modulus [MPa] \\
\hline SG184 & $5.0 \pm 0.5$ \\
SG184(1)-SG186(2) & $3.4 \pm 0.3$ \\
SG184(1)-SG186(3) & $3.1 \pm 0.2$ \\
SG184(1)-SG186(6) & $2.2 \pm 0.2$ \\
SG186 & $1.5 \pm 0.1$ \\
\hline
\end{tabular}

Table 3.1: The elastic modulus of the different PDMS substrates calculated from the Hertzian model.

\subsection{Comparison with the Linear Elasticity Theory}

Even though the previously discussed method provides a quick and easy way to extract the substrate's elasticity by approximating the system to a rigid spherical indenter deforming an elastomeric plane, it is valid only for small drop radii, where the substrate deformation can be accurately fitted by a spherical cap. A more precise and complete theory is needed in order to explain theoretically not only the deformation below the droplet, but also the deformation at the dry side of the three-phase contact line and the vertical displacement of the latter, independently of drop size.

Already in 1975, Rusanov [67], based on the theory of elasticity, considered the deformation of the surface of a solid body under the effect of a liquid sessile drop and developed analytical expressions for the complete profile below and around the sessile drop. Considering that a stress vector $\mathbf{P}$ is the combination of the surface tension at the TPCL and the capillary pressure at the liquid-substrate interface, he calculated the vector $\mathbf{z}(x, y)$ of the vertical displacement at all points of the surface by using the Theory of Linear Elasticity. Projecting his calculations in a $1 \mathrm{D}$ representation in the absence of a gravitational field, Rusanov extrapolated the surface profile $z(x)$ for three sections: the deformation underneath the drop $(x \leq r)$, the deformation at the TPCL, where 
the TPCL is assumed to be a thin line of finite thickness $t(r \leq x \leq r+t)$ and the deformation of the surface not covered by the drop $(x \geq r+t)$.

$$
\begin{aligned}
& z(x)= \\
& -\frac{4\left(1-\nu^{2}\right)}{\pi E}\left\{\Delta \operatorname{Pr} E\left(\frac{x}{r}\right)+\frac{\gamma_{L V} \sin \theta}{t}\left[r E\left(\frac{x}{r}\right)-(r+t) E\left(\frac{x}{r+t}\right)\right]\right. \\
& \left.+\frac{\pi(1-2 \nu)}{4(1-\nu)} \gamma_{L V} \Delta \cos \theta\right\}, \quad x \leq r \\
& z(x)= \\
& -\frac{4\left(1-\nu^{2}\right)}{\pi E}\left\{\Delta \operatorname{Pr} G\left(\frac{r}{x}\right)+\frac{\gamma_{L V} \sin \theta}{t}\left[r G\left(\frac{r}{x}\right)-(r+t) E\left(\frac{x}{r+t}\right)\right]\right. \\
& \left.+\frac{\pi(1-2 \nu)}{4(1-\nu)} \gamma_{L V} \Delta \cos \theta(r+t-x)\right\}, \quad r \leq x \leq r+t \\
& z(x)=-\frac{4\left(1-\nu^{2}\right)}{\pi E}\left\{\Delta P x G\left(\frac{r}{x}\right)+\frac{\gamma_{L V} \sin \theta}{t} x\left[G\left(\frac{r}{x}\right)-G\left(\frac{r+t}{x}\right)\right]\right\}, \quad x \geq r+t
\end{aligned}
$$

where $G(k) \equiv E(k)-\left(1-k^{2}\right) K(k)$ and $E(k)$ and $K(k)$ are the total normal elliptical Legendre integrals of the first and second kind respectively, $\Delta \cos \theta \equiv \cos \theta-\cos \theta_{0}$ is the difference between the macroscopic contact angle and the equilibrium contact angle given by Young's equation, $t=\tau / \sin \theta$, where $\tau$ is the thickness of the precursor film, $\nu$ is Poisson's ratio of the substrate, $r$ is the contact radius of the drop, $E$ is the substrate's Young's modulus and $\Delta P$ is the Laplace pressure inside the sessile drop.

\section{Deformation at the contact area}

In order to compare the shape of the PS/PDMS interface with the theoretical predictions, we use Equation 3.7. In Equation 3.7 we have considered $\Delta \cos \theta \sim 0$, because the macroscopic contact angle is expected to be equal or very close to the equilibrium contact angle, as no significant change in the contact angle was observed when it was measured on the different substrates. In Figure 3.4 is shown the comparison between the experi- 
mentally derived deformation of a PDMS substrate (mixture 1:6 of SG184:SG186) and the theoretical curve derived from Equation 3.7. The same procedure has been followed for all PDMS substrates with equally good agreement between theory and experiment.

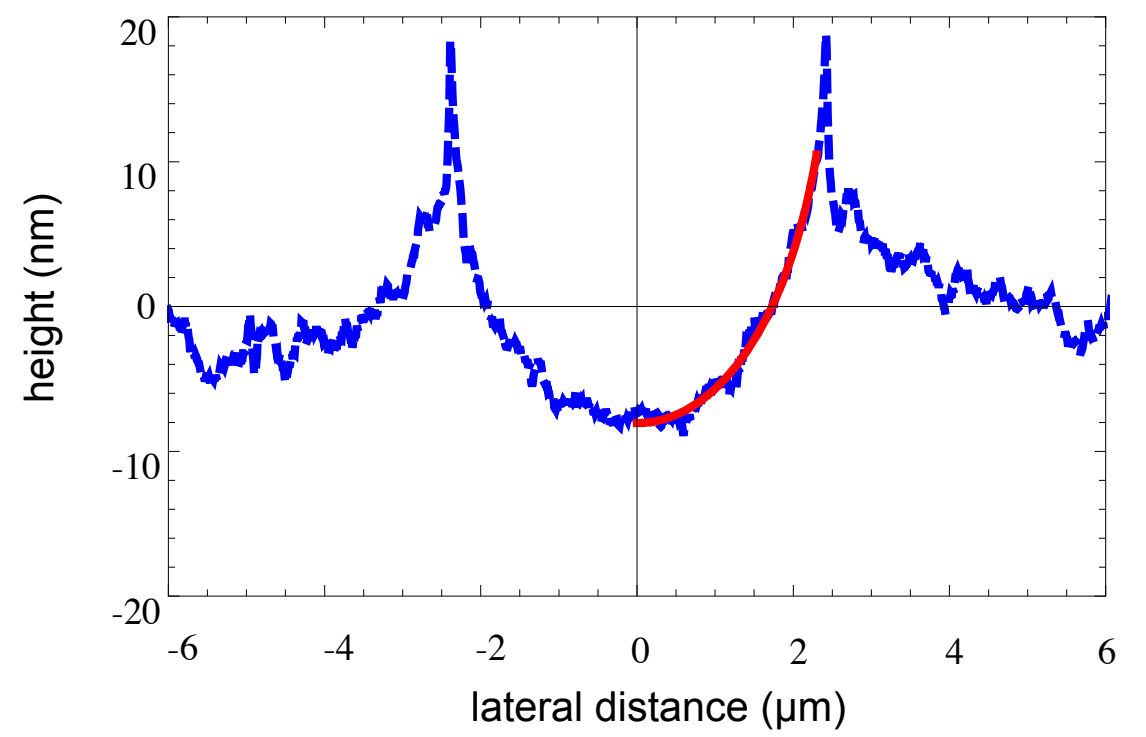

Figure 3.4: Theoretical and experimental profile of a deformed PDMS substrate (mixture 1:6 of SG184:SG186) below a sessile PS droplet. The blue dashed curve represents the experimental profile obtained by AFM, whereas the red solid line is the calculated profile according to Rusanov's model (Equation 3.7).

\section{Deformation at the dry side of the three phase contact line}

The shape of the deformation at the dry side of the TPCL is equally essential in order to reconstruct the complete droplet profile and understand the stresses that the droplet applies on the substrate. In Figure 3.5 is depicted the experimental profile of the substrate deformation at the dry side of the TPCL and the corresponding theoretical profile based on Rusanov's theory calculated from Equation 3.8. Again here the same procedure has been followed for all PDMS substrates with equally good agreement between theory and experiment. 


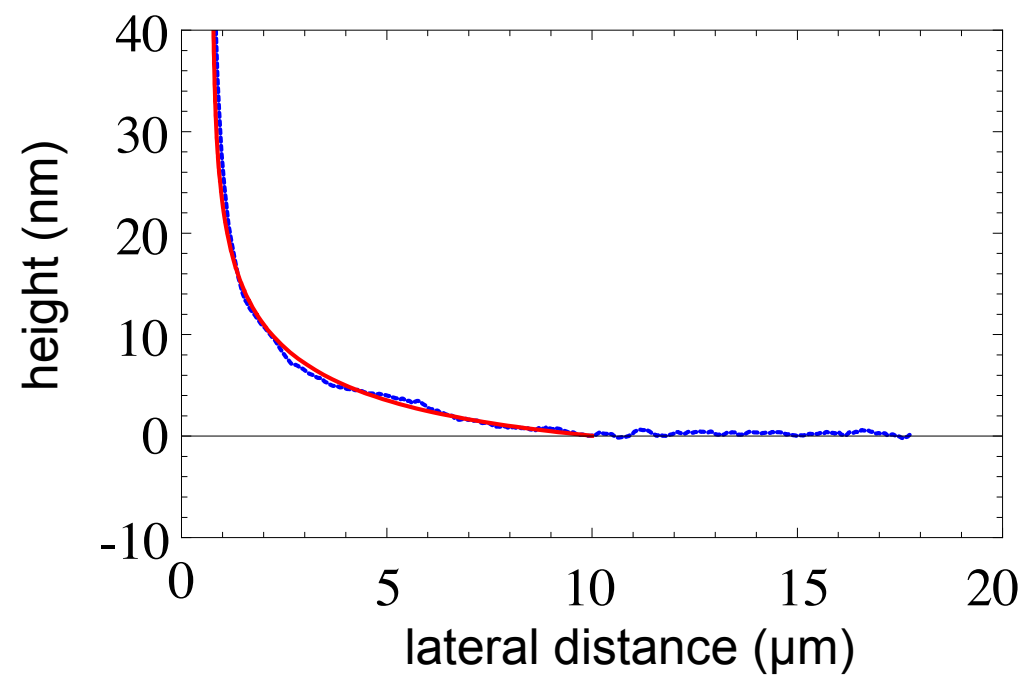

Figure 3.5: Theoretical and experimental profile of a deformed PDMS substrate (SG186) at the dry side of the TPCL. The blue curve represents the experimental profile obtained by AFM, whereas the red solid line is the calculated profile according to Rusanov's model (Equation 3.8).

\section{Extraction of the substrate elastic modulus independent of drop size}

The above procedure of comparing the experimentally derived profiles of the substrate deformation with the given theory, applied for all our elastic substrates, can be used to calculate their elastic modulus. Specifically, using Equation 3.7 and Equation 3.8 as fitting functions with the elastic modulus $E$ being the only free parameter, we end up with two values for the elasticity of each substrate: the value extracted from the deformation below the droplet and the value from the fitting function of the TPCL. Averaging these values, we derive the elastic modulus of our substrates based on Rusanov's model. Our results are given in the second column of Table 3.2 in comparison with the values extracted from the Hertzian model, discussed previously.

From Table 3.2, we can conclude that the two methods, in the range of droplet size used, give results that are in good agreement. The method based on the Hertzian model, due to its simplicity, is preferable for small droplet sizes, where the substrate deformation exhibits a spherical cap shape. Nevertheless, for larger droplet sizes, where 


\begin{tabular}{|c|c|c|}
\hline PDMS substrate & $\begin{array}{c}E \text { modulus [MPa] } \\
\text { (Rusanov) }\end{array}$ & $\begin{array}{c}E \text { modulus [MPa] } \\
\text { (Hertz) }\end{array}$ \\
\hline SG184 & $4.8 \pm 0.3$ & $5.0 \pm 0.5$ \\
SG184(1)-SG186(2) & $3.9 \pm 0.4$ & $3.4 \pm 0.3$ \\
SG184(1)-SG186(3) & $3.6 \pm 0.5$ & $3.1 \pm 0.2$ \\
SG184(1)-SG186(6) & $2.6 \pm 0.5$ & $2.2 \pm 0.2$ \\
SG186 & $2.1 \pm 0.5$ & $1.5 \pm 0.1$ \\
\hline
\end{tabular}

Table 3.2: The elastic modulus of the different PDMS substrates calculated from Rusanov's model (left column) and from the Hertzian model (left column).

the substrate deformation is not spherical anymore, but it rather presents a flat area in the center, the use of the more elaborate model of Rusanov is recommended.

\section{Vertical displacement of the three phase contact line}

Determining the position of the TPCL is a rather complicated task. One could achieve it by analysing the "bottom side" of the droplet as has been done for the deformation of the PS/PDMS interface in Figure 3.4. Nevertheless, in this case, a UV curable glue has been employed to lift-off the PS droplets that might have affected even slightly the deformable PDMS. Thus, when analysing the "bottom side" of the PS liquid morphologies, especially in the case of such a sensitive measurement as the locating of the TPCL, only the data that correspond to the glassy PS surface can be considered absolutely reliable. Thus, to locate the TPCL, we can only use the "top side" of the droplet (Figure 3.3. (a)). The air/PS interface is scanned using the HarmoniX ${ }^{\mathrm{TM}}$ Nanoscale Material Property Mapping Mode (Veeco, Santa Barbara, USA), which, in addition to the signals obtained from the classical Tapping Mode ${ }^{\mathrm{TM}}$ also provides a signal for the elasticity of the material scanned, which is independent of the surface topography, as opposed to the phase signal, which is often affected by the topography when the latter is very sharp or when the scan rate is not sufficiently low.

As can be seen in Figure 3.6, by extracting contemporaneously from the AFM scans the topography and elasticity signals, we are able to preform a direct correlation of the two, which allows us to identify the region that corresponds to the undisturbed PDMS substrate (left region in Figure 3.6. (b)), the region of higher elasticity that corresponds to the glassy PS (right region in Figure 3.6.(b)) and the region where the elastic modulus 
changes rapidly that corresponds to the PDMS ridge (middle region in Figure 3.6. (b). The exact point of the TPCL can be identified as the point joining the end of the deformed PDMS and the beginning of the PS droplet.

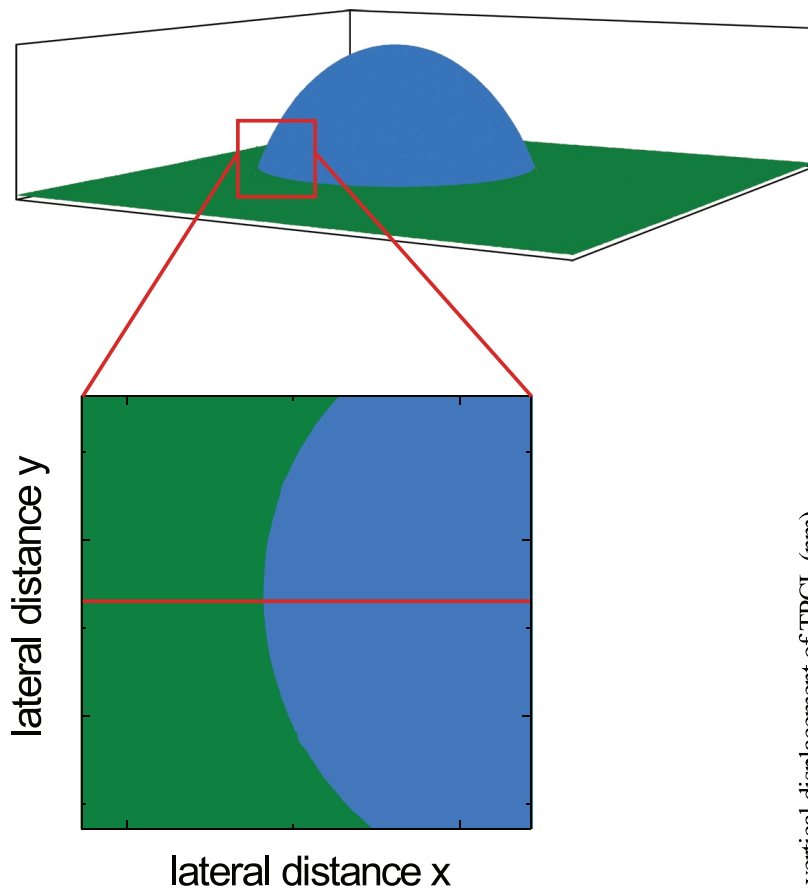

(a)

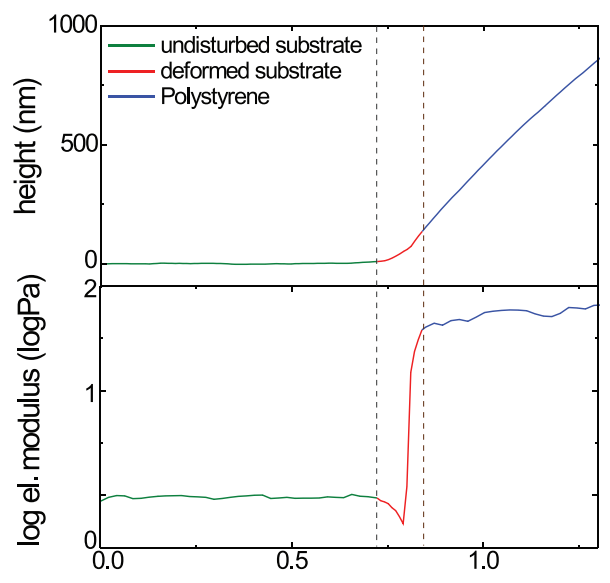

(b)

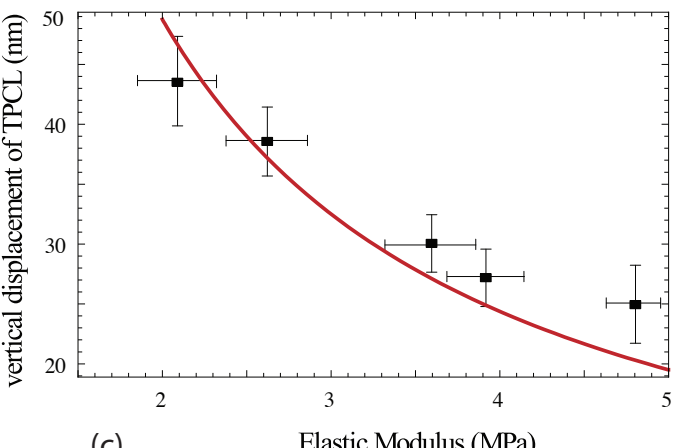

(c)

Figure 3.6: (a) Top: 3d surface plot of an AFM scan of a PS droplet (blue) on a rubber substrate of PDMS (green) and a highlight of the region of interest (ROI). Bottom: Top view of an AFM scan of the ROI and a cross-section (red line). (b) Graphs of the cross-sections of AFM scans of the height and elasticity signals. (c) Plot of the vertical displacement of the TPCL as a function of substrate elasticity (data points) and the theoretical curve calculated from Rusanov's model (solid line). The values for the elasticities used in this plot are calculated based on Rusanov's model reported in the first column of Table 3.2 .

Performing a similar analysis for all our substrates, we can extract and plot the vertical displacement of the TPCL as a function of the substrate elasticity (Figure 3.6.(c)). Our results are plotted together with the theoretical curve calculated from Equation 3.8 by Rusanov for $x=r+t / 2, t=10^{-10} \mathrm{~m}$. 
In parallel to locating the position of the TPCL, with this technique one can also extract the elastic modulus of the substrates. To do that, the geometry and physical properties of the tip have to be known and the elasticity signal has to be normalized using a standard. For our system, an absolute value of the elastic moduli was not extracted, as it would be unreliable. That is because the order of magnitude of the elasticity of PDMS is roughly a factor of two lower than the lower limit of elasticity that can be measured by HarmoniX with the available probes, as reported by the manufacturer. Nevertheless, we can still extract relative values, by comparing the elasticity signals given from the bottom graph of Figure 3.6.(b) for different substrates, if we consider that the PS droplets on the different types of PDMS always have the same elastic modulus. Thus, comparing the elastic modulus signals for SG184 and SG186, we find that the ratio of their elasticities is $3.0 \pm 0.1$, SG184 being the stiffest one, which is in very good agreement with the values given in Table 3.2 . 


\section{Chapter 4}

\section{Dewetting on rubber elastic substrates}

Having analysed in Chapter 3 the static case of a PS droplet in equilibrium on a rubber elastic substrate, we can now take a chronological step back and examine the mechanism that generates these droplets, i.e. the dynamics of a PS thin film dewetting on a rubber elastic substrate.

\subsection{State of the art - Dewetting on rigid substrates}

We will restrict this short overview to a summary of some of the most important aspects of dewetting on rigid substrates and specifically the dynamics involved in the dewetting process and its impact on the shape of the rim profiles.

In the typical dewetting scenario, a rupture mechanism induces isolated holes to pop up in an elsewhere quite uniform film. The material removed from the inside of the hole is accumulated in a rim surrounding the hole and the liquid polymer forms a finite contact angle with the substrate, which is characteristic for the system [68]. When a hole meets its neighbouring holes, it cannot grow any further and the rims of these holes will overlap forming a common liquid ribbon, which is dynamically unstable and decays via a Rayleigh-Plateau instability into single droplets. Thus, the resulting pattern is a polygonal network made up of droplets. 
For the dewetting on a rigid solid substrate, the mechanism that controls the dewetting speed is relatively well known [2, 33] and is determined essentially by a dynamic energy balance between the free surface/interfacial energy and the energy released by the dewetting process. The driving force for the growth of the hole is the negative spreading coefficient,

$$
S=\gamma_{L V}\left(\cos \vartheta_{e}-1\right)
$$

where $\gamma_{L V}$ is the surface tension of the air/liquid interface of the dewetting liquid and $\vartheta_{e}$ is the equilibrium contact angle of the dewetting liquid on the substrate. On the other hand, energy is dissipated both by viscous flow within the rim of the hole (Figure 4.1. (a)) and by viscous friction (Figure 4.1.(b)) confined at the liquid/solid interface as the film slips on the substrate [18]. The amount of energy dissipation distributed between these two mechanisms changes with the film thickness and the size of the hole. Specifically in a small rim (either for a small hole or for a rim in a very thin film) slippage plays a prominent role. Since the volume of the rim is determined by the conservation law for the liquid volume, as the rim grows, the area of slippage at the liquid/solid interface increases too, leading to an $r \propto t^{2 / 3}$ (Figure 4.1.(c)) dependence and an asymmetric rim shape. As the rim continues to grow, it eventually shifts to a weak- or no-slip regime, where viscous dissipation dominates the hole growth and $r \propto t$ (Figure 4.1.(c)) resulting in a rather symmetric rim profile [69, 70]. In [2] Jacobs et al. derive an expression for the temporal development of circular holes, which yields the limiting cases of pure viscous flow and full slippage of the film on the rigid substrate. Moreover, they determine a theoretical growth law, which is in good agreement with the experimental results and which can be used to characterize the interplay in the system between viscous flow and sliding friction [2, 71].

From the above it is clear that slip effects, respectively friction at the interface, may dominate the dynamics and control morphological instabilities that occur in dewetting. Fetzer and al. [72] studied the influence of slippage on the dewetting dynamics by comparing the flow behavior of liquid polymer films on silicon wafers coated with different self-assembled monolayers (SAMs), changing thus the short range interactions at the solid/liquid interface and keeping all other parameters of the system fixed. Their main results are summarized in Figure 4.2. Their experiments show that, for the thicker monolayer (Octadecyltrichlorosilane - OTS), the shape of the rim develops a damped oscillatory structure towards the undisturbed film, while for the thinner one (Dodecyl- 
(a)

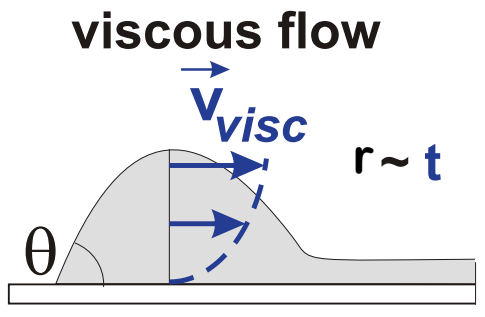

(b)

friction at interface

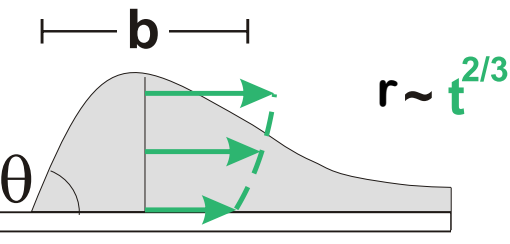

(c)

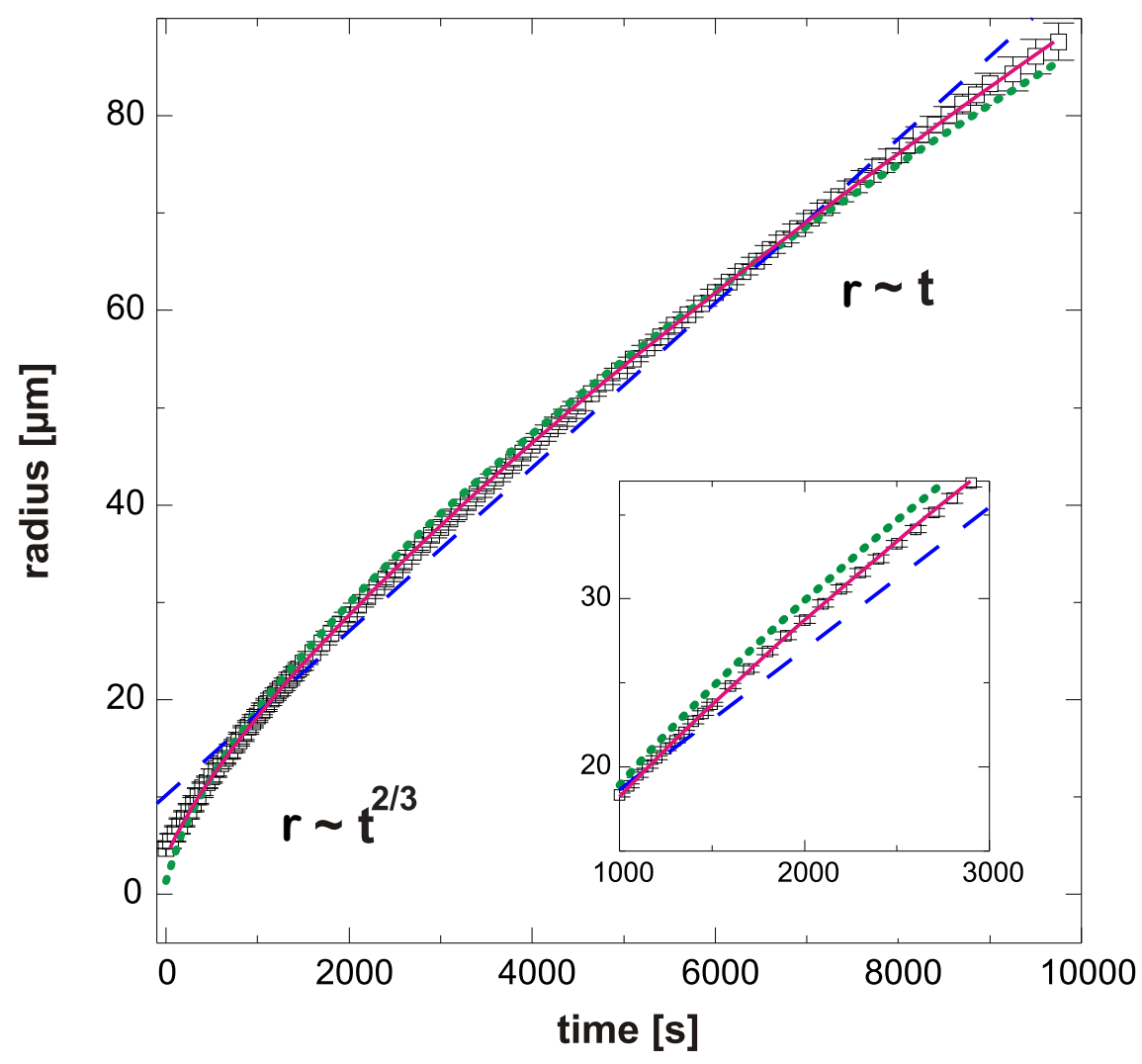

Figure 4.1: (a)-(b): Sketch of the flow profiles of the two possible mechanisms of energy dissipation and the growth law of the holes. $r$ denotes the hole radius and $t$ the annealing time. (c): Typical behavior of the hole radius as a function of time. Blue dashed curve: the theoretical prediction for the case where energy dissipation is dominated by viscous flow. Green dotted curve: the theoretical prediction for the case where dissipation takes place only by friction at the interface. Red solid curve: theory [2] fitted to the experimental data for the real case where both viscous flow and friction at the interface are present. (Image courtesy of K. Jacobs.) 


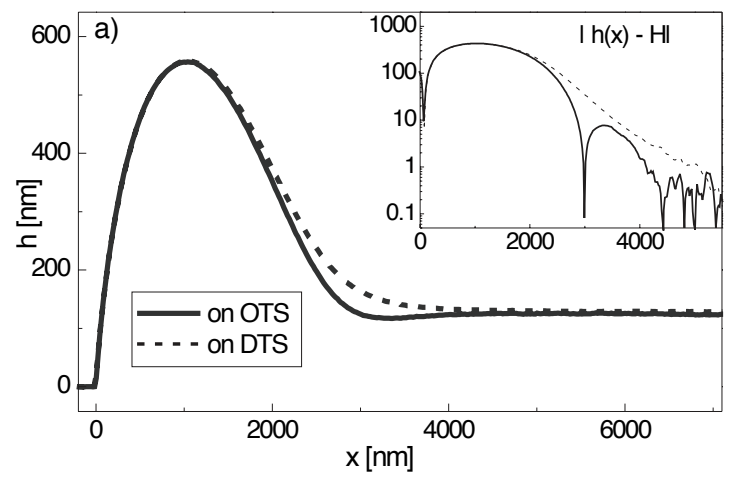

(a)

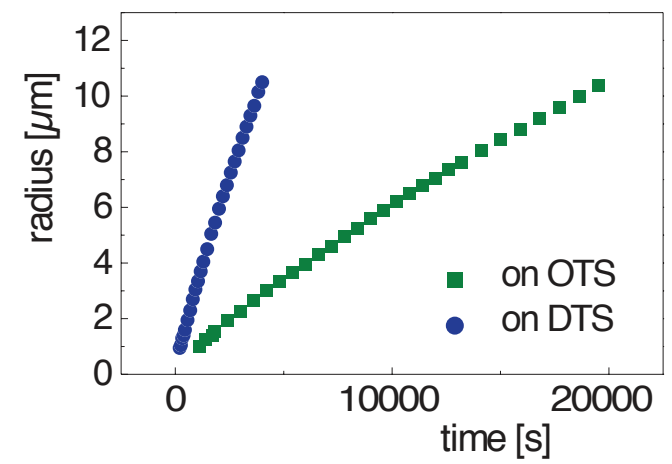

(b)

Figure 4.2: (a) Rim profiles of a $130 \mathrm{~nm}$ PS film on OTS and DTS covered Si wafers. The inset depicts a semilog plot of $|h(x)-H|$, where $H$ is the PS film thickness and (b) the corresponding dewetting rates on the two different substrates. (Images courtesy of R. Fetzer)

trichlorosilane - DTS) the rim decays monotonically. This behaviour can be explained by analysing the dewetting rates, which are significantly higher for DTS, indicating a lower friction coefficient and hence a higher slippage for this coating, which suppresses the spatially oscillatory structure on the side of the rim facing the undisturbed film. The above considerations were verified by a mathematical model developed by A. Münch and B. Wagner [72], which is able to predict the above experimental findings on the basis of purely viscous and slippage effects.

Taking into account the foregoing discussion, our aim in the following sections is to investigate the role of the deformability of the viscoelastic substrates on the dewetting dynamics. 


\subsection{Dewetting dynamics on rubber elastic substrates}

\subsubsection{Dewetting rates}

In this section, we will concentrate mainly on the dynamics of growth of the circular holes, the impact of the substrate elasticity on the rim profiles and its influence on the shape of the underlying deformation.

The dewetting process was started by annealing the sample above the glass transition temperature $T_{g}$ of the polystyrene. After a few minutes of annealing, circular holes appear on the film and grow with time. The dewetting process is monitored in situ by reflection microscopy and recorded by a CCD-camera (Figure 4.3.(a)). Subsequently, the hole growth is analysed as a function of time. The dewetting rates for the different substrates are shown in Figure 4.3.(b). The elasticities of the substrates have been calculated in Chapter 3 and are given in Table 3.2. It is readily seen that the dewetting of polystyrene progresses faster on the stiffer SG184 substrate and gradually slows down as the rubber gets softer, i.e. for SG184 mixed with larger quantities of SG186, in order to finally reach the lowest dewetting rates for pure SG186.

In order to preclude that this result might be due to any viscous properties of the substrate stemming from a possible incomplete cross-linking during preparation, we perform several tests that include leaching the PDMS substrates before picking-up the PS film and using different curing times, but no considerable difference in the dewetting rates was observed. The details and results of this analysis are given in Appendix A.

This finding of the decreasing dewetting rates with decreasing substrate elasticity can be correlated to the results of Carré and Shanahan on the viscoelastic breaking of a running drop [73]. In a series of experiments they performed, a sessile droplet is running down an inclined elastomeric plane. The speed of the droplet is measured for different liquid volumes, angles of inclination and substrate elasticities. What is found is that, for a specific liquid volume and angle of inclination, the velocity with which the droplet moves on the elastomeric surface is dependant on the substrate elasticity and specifically it decreases with decreasing substrate elasticity. They attribute this phenomenon to the wetting ridge caused by the vertical displacement of the three-phase contact line due 


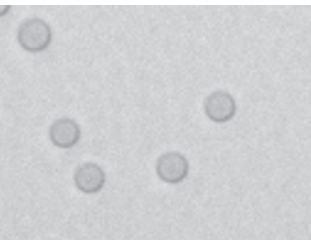

(a)
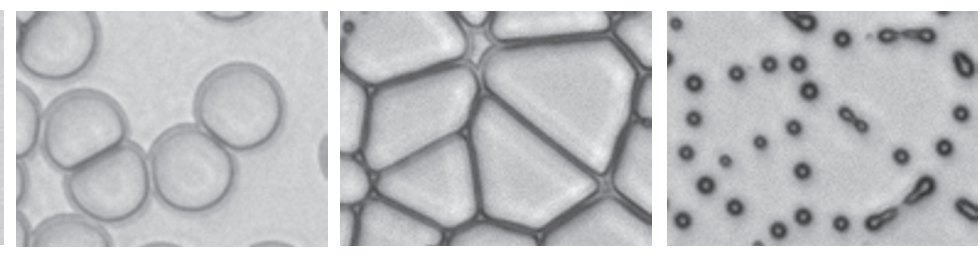

$10 \mu \mathrm{m}$

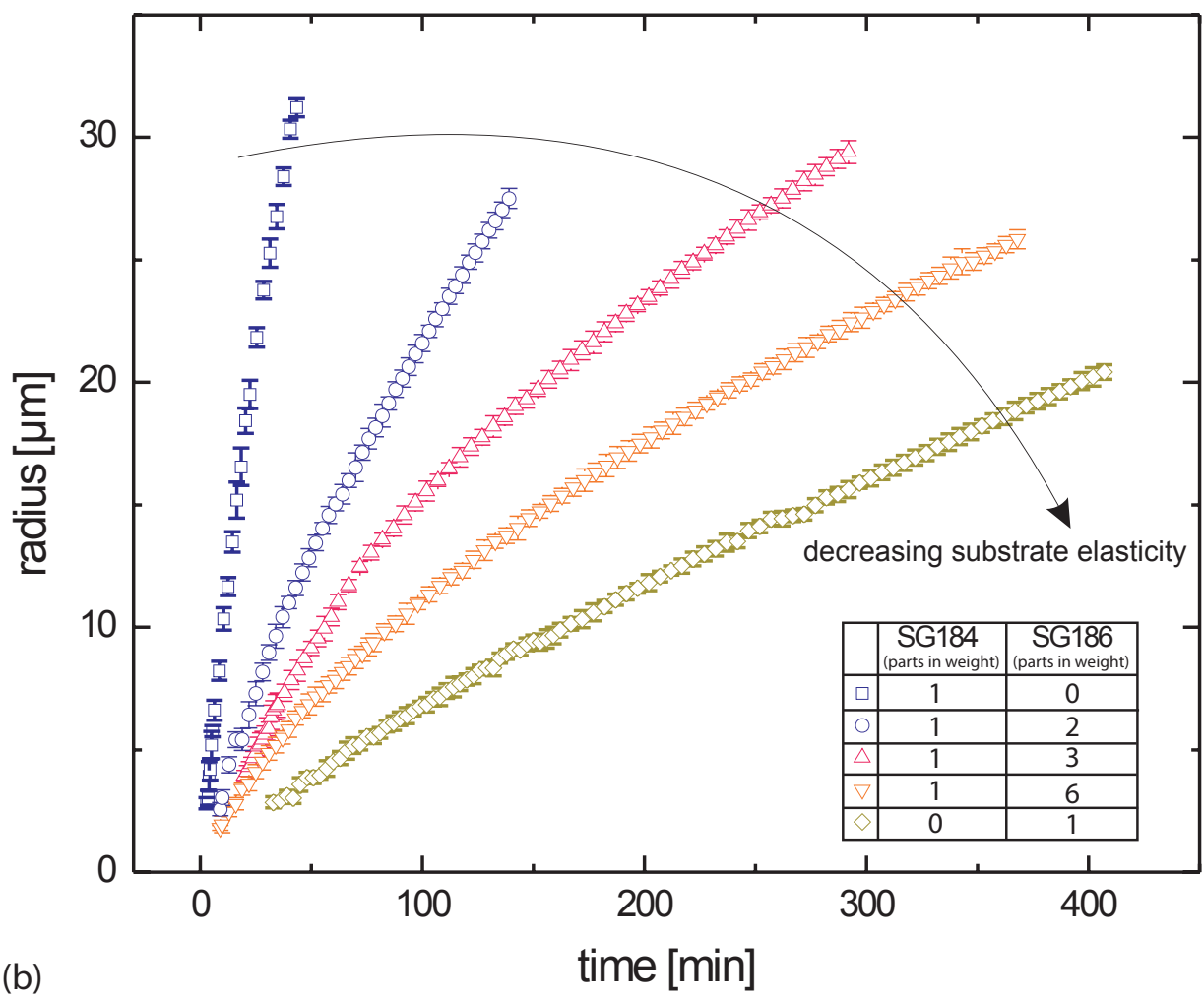

Figure 4.3: (a) Time series of a PS(17.4k) film with a film thickness of $150 \mathrm{~nm}$ dewetting at $\mathrm{T}=120^{\circ}$ on SG184. (b) Dewetting rates for a $170 \mathrm{~nm}$ thick PS(17.4k) film at $120^{\circ} \mathrm{C}$ dewetting on substrates of different elasticities. In the tabular are given the ratios with which SG184 and SG186 are mixed in order to produce substrates with intermediate elasticities.

to the liquid surface tension $\gamma_{L V} \sin \theta_{e}$, discussed previously in Chapter 3 . According to their theory, as the dewetting front advances, so does the accompanying deformation and negative work is done by deforming the solid. For a perfectly elastic substrate, 
after the dewetting front has moved forward, the surface is released, and no net work is done. If, however, the deformable solid possesses hysteretic strain characteristics, its continuous deformation and subsequent release will have a significant influence on the wetting/dewetting dynamics [74, 75].

Regarding the shape of the single curves, it resembles the one obtained for the real case of a polymer dewetting on a rigid solid substrate (experimental data in Figure 4.1.(c)), i.e. for all substrate elasticities, the dewetting is faster for small holes and slows down as the hole, respectively the width of the dewetting rim, grows. The fact that the dewetting velocity does not remain constant with time, implies that energy is not dissipated exclusively at the three-phase contact line. Energy must be dissipated also at the liquid/rubber interface of the rim, which is expected to be deformed under the stress of the dewetting liquid film.

Given that the elasticities of the substrates are known as they have been calculated in Chapter 3, we can now examine the behaviour of the dewetting velocity as a function of substrate elasticity. In Figure 4.4 we plot the dewetting velocity of a $150 \mathrm{~nm}$ thick PS(17.4k) film dewetting at $120^{\circ} \mathrm{C}$ on PDMS as a function of the PDMS elastic moduli, which have been calculated based on the Hertzian model. The values for the velocities

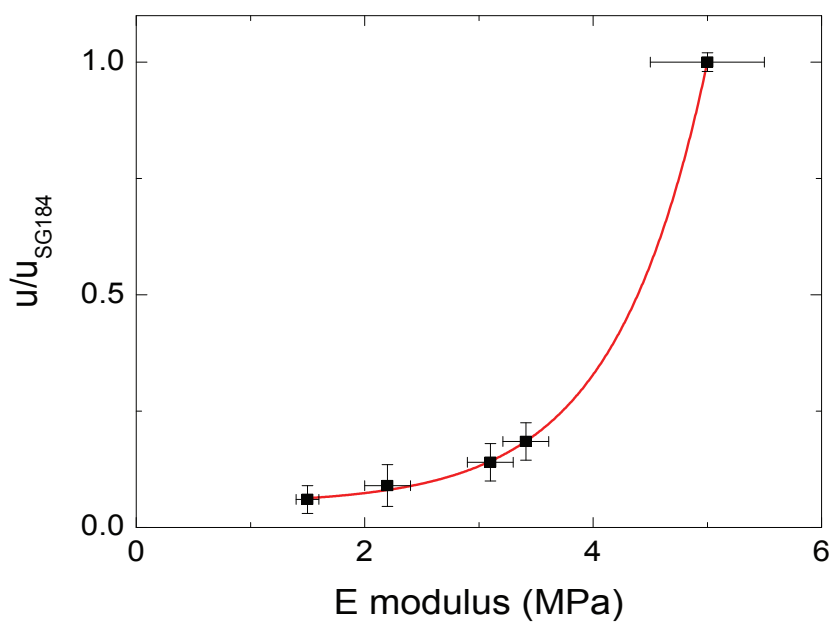

Figure 4.4: Normalized dewetting velocities of a PS(17.4k) film with thickness of $150 \mathrm{~nm}$ dewetting at $\mathrm{T}=120^{\circ}$ on substrates of different elasticities as a function of the substrate elasticity (data points) and the exponential fit to the experimental results (solid curve). 
have been calculated from the first derivative of the linear part of the dewetting rates shown in Figure 4.3. Subsequently, they have been normalized with respect to the velocity of SG184. The red curve is the fit of the data points to an exponential growth, which expresses the sensibility of the dewetting velocity on even small variations of the substrate elasticity. The exponential fit though, is valid only within a certain region of the substrate elasticity. If we extend the x-axis of the substrate elasticity to higher values, we expect the exponential fit to "transform" into a sigmoidal function, as, above a certain threshold, the overlying film will "feel" a rigid substrate and the increase in substrate elasticity will not play a role anymore. Thus the dewetting velocity will be independent of the substrate elasticity.

\subsubsection{Rim profile}

The next step in the investigation of the influence of the substrate elasticity on the dewetting dynamics is to study the morphology of the dewetting rim, both the air/PS and the PS/PDMS interface. To do that, we continued with the annealing of the dewetting film as described above and, once the holes had reached the desired radius, we immediately quenched our samples to room temperature in order to bring the polystyrene film in the glassy state and imaged the air/PS interface by AFM in Tapping mode ${ }^{\mathrm{TM}}$. The ex-situ imaging of the rims was adopted for practical and simplicity purposes, as previous studies have revealed that rim profiles imaged at $T<T_{g}$ do not present any significant differences from the ones imaged at $T>T_{g}$ [35, 72].

A radial cross section of a typical rim surrounding a hole on a PS film dewetting on PDMS is shown in the graph of Figure 4.5. (c). The air/PS interface of the rim is found to be rather symmetric, forming a dynamic contact angle $\theta_{d}$ with the substrate that is very close to the equilibrium contact angle $\theta_{e}$. The height $h$ of the rim is measured between the free air/PDMS interface and the peak of the rim, while two different measurements are taken in order to characterize the width of the rim: the full width at half maximum FWHM, maximum being the distance between the undisturbed air/PS interface and the peak of the rim and $W$, the width of the rim measured between the three-phase contact line and the part of the rim that advances into the undisturbed film. Exactly at that point, one can observe a "trough" of depth $d_{t}$, a structure that resembles a damped harmonic oscillation. Regarding the deformation that the liquid rim causes 

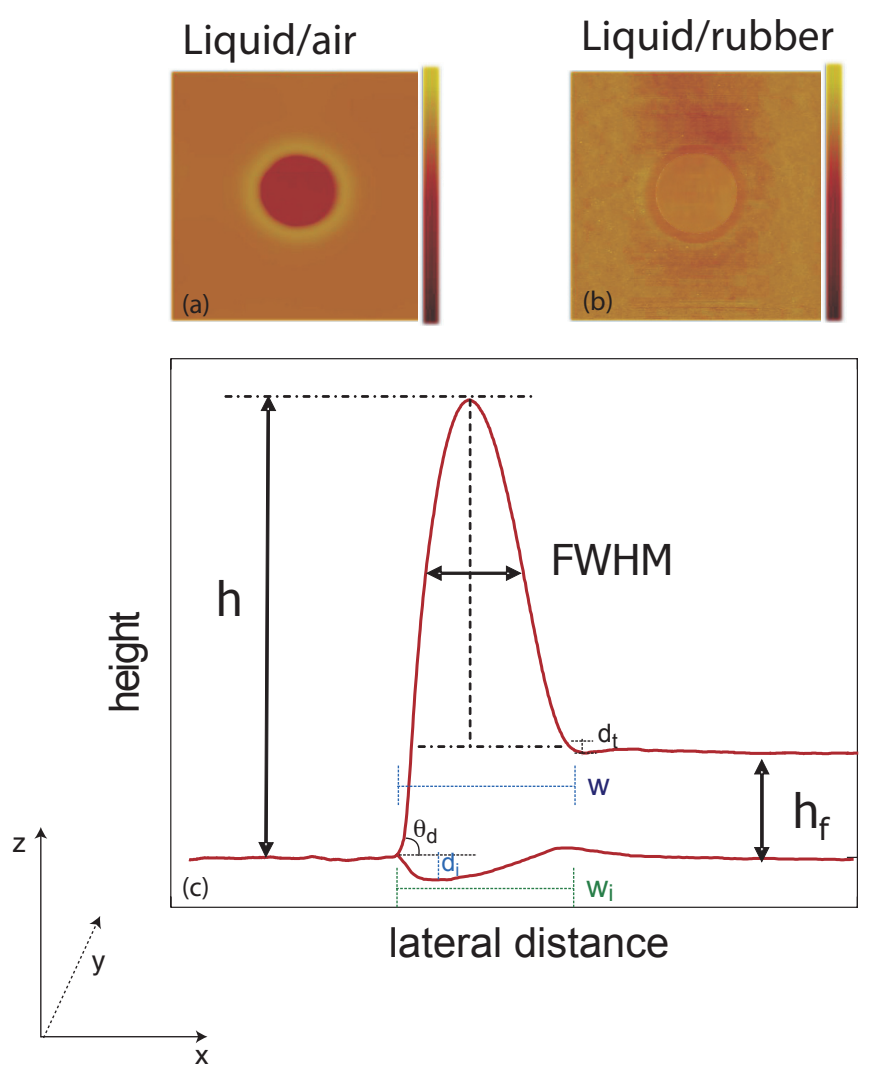

Figure 4.5: (a) AFM scan of the liquid/air interface of a typical hole of PS dewetting on PDMS, (b) AFM scan corresponding to the liquid/rubber interface around the hole, (c) Radial cross section of the hole and the corresponding substrate deformation.

on the substrate, we observe the following: below the symmetric rim profile, we find the presence of a depression of depth $d_{i}$ that attempts to "mirror" the overlying rim profile. Accordingly, right below the trough of the air/PS interface a protuberance of the PS/PDMS interface can be observed.

To understand how these characteristic features develop with time, we image by AFM a hole growing with time as seen in the series of cross-sections of AFM scans in Figure 4.6. To obtain this "time series", we performed several dewetting experiments on the same type of samples using identical parameters and stopped the annealing when the holes had reached the desired radius. At that point, the air/PS and the corresponding PS/PDMS interface were imaged.

Examining the development of the rim, we observe that the wet side of the TPCL 


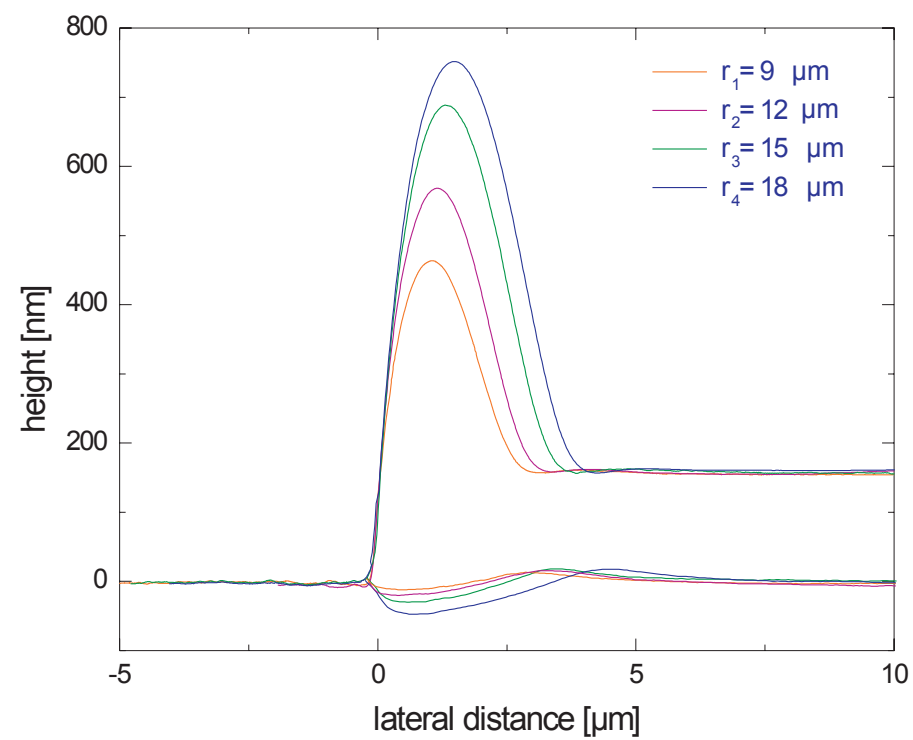

Figure 4.6: Cross-sections of AFM scans of rim profiles at increasing annealing time. The hole radii are $9 \mu \mathrm{m}, 12 \mu \mathrm{m}, 15 \mu \mathrm{m}$ and $18 \mu \mathrm{m}$ in $150 \mathrm{~nm}$ thick PS(17.4k) films dewetting on SG186 at $120^{\circ} \mathrm{C}$.

overlaps perfectly for all rims, i.e. the contact angle $\theta_{d}$, which is calculated from the slope at the TPCL, stays constant with time. Accordingly, the slope at the wet side of the rim, with the exception for the first hole where the rim might not be fully developed yet, also stays constant with time. This means that the rim, which can be fitted to a spherical cap, undergoes an affine expansion during the dewetting process. Furthermore, as the rim grows with time, so does the indentation depth of the deformed substrate. Finally, the trough towards the undisturbed side of the film moves along as the rim grows, followed by the corresponding protuberance at the PS/PDMS interface. This protuberance might be generated in order to balance the high Laplace pressure that the large curvature of the trough is creating at that point of the air/liquid interface of the liquid film.

To quantify the above affirmations, in Figure 4.7 we have plotted the behaviour of the rim features as a function of hole radius, respectively time. Graph (a) has been plotted based on data extracted from several experiments of PS films of different thicknesses dewetting under the same conditions on SG186. In order to be able to compare these data, we have normalized them with respect to the film thickness. One can observe that 

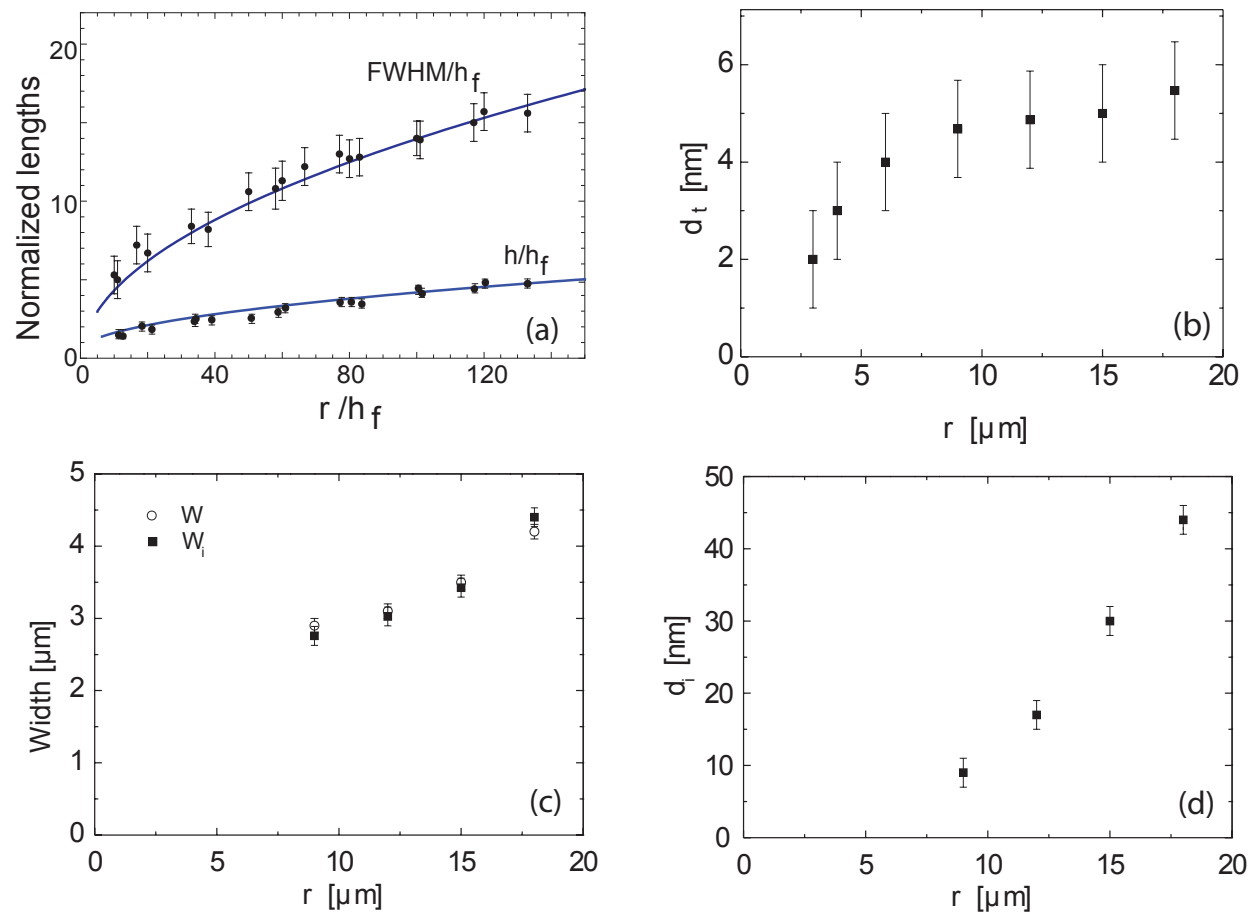

Figure 4.7: (a) Rim height $h$ and Full Width at Half Maximum $F W H M$ as a function of the normalized hole radius (b) depth $d_{t}$ of the undulation as a function of hole radius, (c) width $W$ of the upper part of the rim and width $W_{i}$ of the substrate deformation as a function of hole radius and $(\mathrm{d})$ indentation depth $d_{i}$ of the deformed substrate as a function of hole radius.

both the rim height and $F W H M$ data collapse into two single curves, which correspond to the theoretical curves for the rim height and $F W H M$ respectively calculated from the mass conservation of the liquid volume that formerly covered the inside of the hole and is now surrounding the hole in a rim.

This theoretical curve is calculated making the following assumptions: the interior of the hole can be approximated to an inverted truncated cone with bottom radius $r$ equal to the hole radius, opening angle $\pi-\theta_{e}$ and height equal to the film thickness $h_{f}$. In parallel, the rim surrounding the hole can be approximated to a truncated ring torus generated as the product of a circle of radius $r$ corresponding to the radius of the circular fit of the air/PS interface of the rim with a circle of radius $r+W / 2$. According to the 
mass conservation of the liquid volume, the volume of the cone at every given time $t$ must equal the volume of the torus. From this equation the theoretical prediction of the height and width of the rim can be extrapolated. A more precise calculation would also involve the difference in the liquid volume due to the substrate deformation, but since this is much smaller than the liquid volumes we are dealing with, it can be neglected and still satisfactory agreement between theory and experiments can be achieved. Figure 4.7. (c) depicts the behaviour of the width $W$ of the upper part of the rim and width $W$ of the deformation as a function of hole radius. The width of the rim increases with time and the two widths increase with the same rate throughout the growth of the hole. Regarding the depth of the trough (Figure 4.7.(b)) at the air/PS interface, initially, for small hole radii, it exhibits a small increase as a function of hole radius, which nevertheless tends to disappear for bigger rims. Finally, the indentation depth $d_{i}$ exhibits a clear increase as a function of rim size (Figure 4.7.(d)), contrary to what has been found for the indentation depth of the static case, which is independent of drop size.

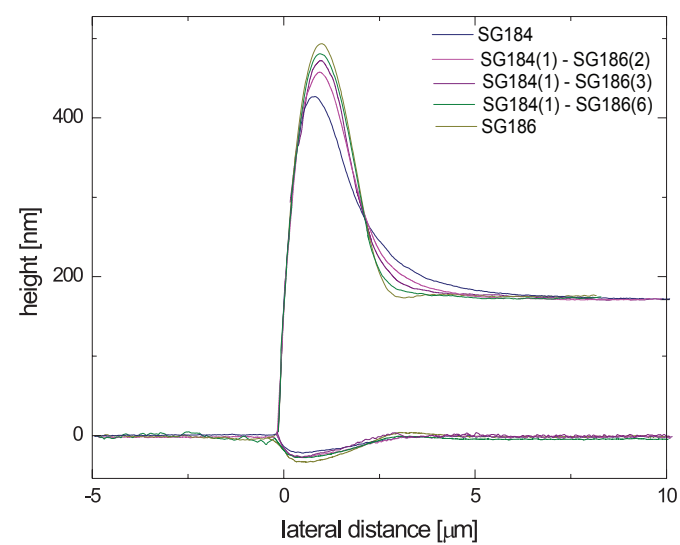

(a)

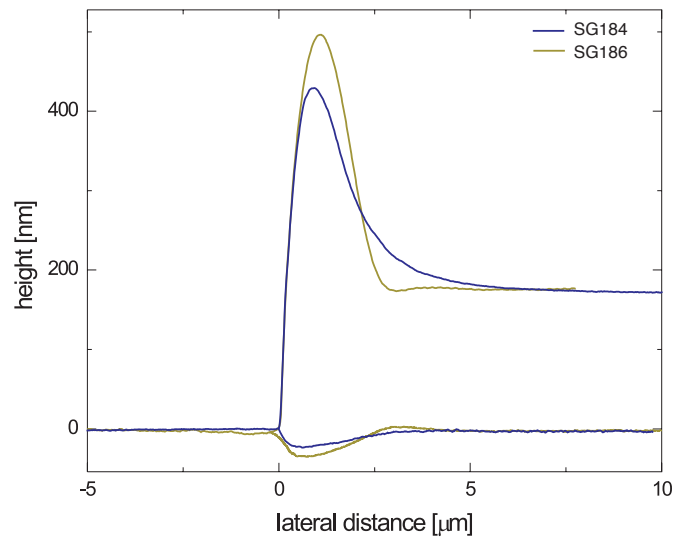

(b)

Figure 4.8: (a) Rim profiles of $\operatorname{PS}(17.4 \mathrm{k})$ dewetting at $120^{\circ} \mathrm{C}$ on substrates of different elasticities. The film thickness and hole radius are kept constant in both systems. (b) Two of the rim profiles of Graph.(a) on SG186 (softest substrate) and SG184 (stiffest substrate).

So far, we have examined how the shape of the rim changes with the growing of the hole on one specific substrate. How does the rim and the corresponding interface 
deformation change with the substrate elasticity? In Figure 4.8.(a) are plotted the dewetting profiles of a $180 \mathrm{~nm}$ thick $\mathrm{PS}(17.4 \mathrm{k})$ film on substrates of different elasticities. The film thickness, the hole radius, and the dewetting temperature are held constant. For the sake of clarity, in Figure 4.8.(b), we report the results only for the two extreme cases of pure SG184 and SG186, but, as can been seen, an analogous behaviour is found for all the substrates used in our study. Regarding the air/PS interface of the rim, it is evident that the rim corresponding to the substrate of lower elasticity (SG186) is more symmetric with respect to the rim corresponding to the higher elasticity substrate (SG184). Furthermore, towards its undisturbed part, the rim exhibits an oscillatory behaviour as we have seen also in the previous images that is suppressed for the rim on SG184, which decays monotonically into the undisturbed film. Taking into account the dewetting rates that correspond to these two systems as shown in Figure 4.3, we can claim that, for the softer substrates, the rim progresses more slowly, is more symmetric and presents a more pronounced damped oscillatory behaviour. The relation between the dewetting rates and the shape of the rim is qualitatively analogous to the one found by Fetzer et al. [72, presented at the beginning of the chapter and summarized in Figure 4.2. In that case, the difference in the dewetting rates and in the shape of the rim profile between the two different substrates was attributed to the higher slip on DTS than on OTS. In our system, the change in the shape of the rim on the two different substrates comes to strengthen the assumption made based on the dewetting rates, i.e. that energy is not dissipated only at the three-phase contact line, but also at the deformed PS/PDMS interface. As far as the PS/PDMS interface is concerned, the indentation depth is larger for the lower elasticity substrate and it is accompanied by a protuberance, which tends to disappear for the higher elasticity substrate, "obeying" the monotonical nature of the decay at the air/PS interface.

\subsubsection{Comparison with a theoretical model}

To explain theoretically the previously discussed results regarding the shape of the rim, we implement the Linear Elasticity Theory. In collaboration with M. Brinkmann, we calculate theoretically the amount of the local deformation that the Laplace pressure of the liquid causes on the substrate and we compare it with our experimental results. The details of the theoretical calculations can be found in Appendix B. 


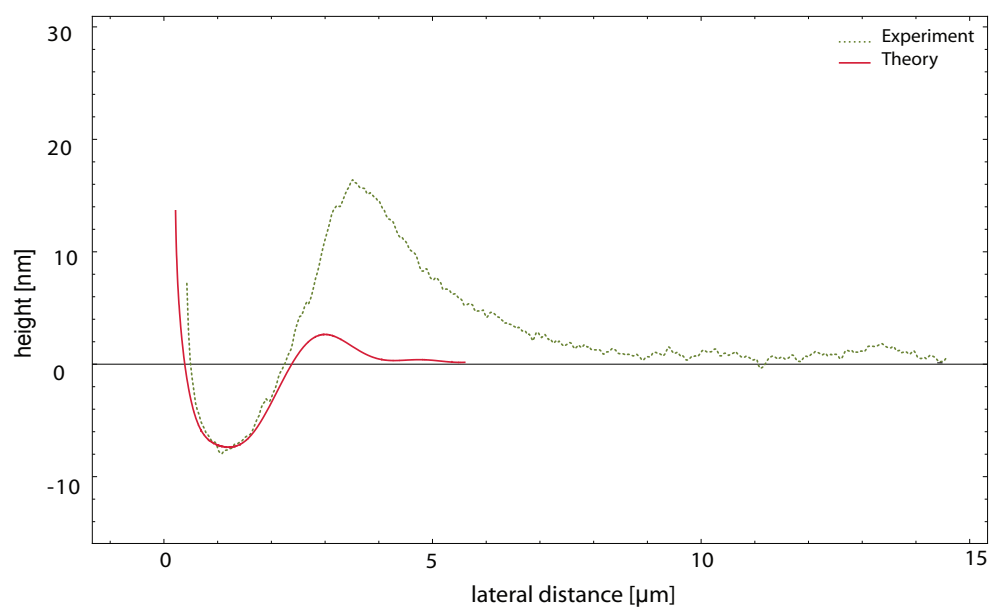

Figure 4.9: Cross section of an AMF scan of the liquid/rubber interface below a rim of PS(17.4k) dewetting on SG186 at $120^{\circ} \mathrm{C}$ (green dotted curve) compared to the deformation calculated taking into consideration only the Laplace pressure inside the liquid (red solid curve). The radius of the hole is $r=9 \mu \mathrm{m}$.

In Figure 4.9 is depicted the PS/PDMS interface below the rim of the hole with radius $r=9 \mu \mathrm{m}$ shown in Figure 4.6 and the corresponding theoretically calculated deformation. We observe that the deformation below the circular arc of the rim is adequately predicted by the theoretical model, whereas the protuberance towards the undisturbed PDMS substrate is found to be larger, both in height and width, than the one calculated by the theoretical model.

Applying the same considerations to the rim of a larger hole $(r=12 \mu \mathrm{m}$ in Figure 4.6), we obtain the graph of Figure 4.10. We observe that, in this case, the deformation below the circular arc of the rim begins to deviate from the one expected theoretically. Furthermore, the deviation between the experimentally measured protuberance towards the undisturbed PDMS substrate and the one calculated theoretically has increased.

This means that, for the smaller hole, the deformation below the circular arc of the rim can be attributed entirely to the Laplace pressure inside the liquid. As the hole, respectively the rim, grows, dynamic effects become more significant and the sole implementation of the Laplace pressure in the model is not sufficient anymore in order to quantify the deformation below the rim. The deviation between the experimental and theoretical protuberance towards the undisturbed PDMS substrate could be moreover attributed to the depletion zone at the dry side of the three-phase contact line that can 
be observed in Figure 4.8 for SG186. Material is "stretched" away from that area and it is "redistributed" in the area below the rim. Since the depletion zone has not been taken into account in the theoretical model, mass conservation in the model accounts for less material below the rim than the amount of material actually present.

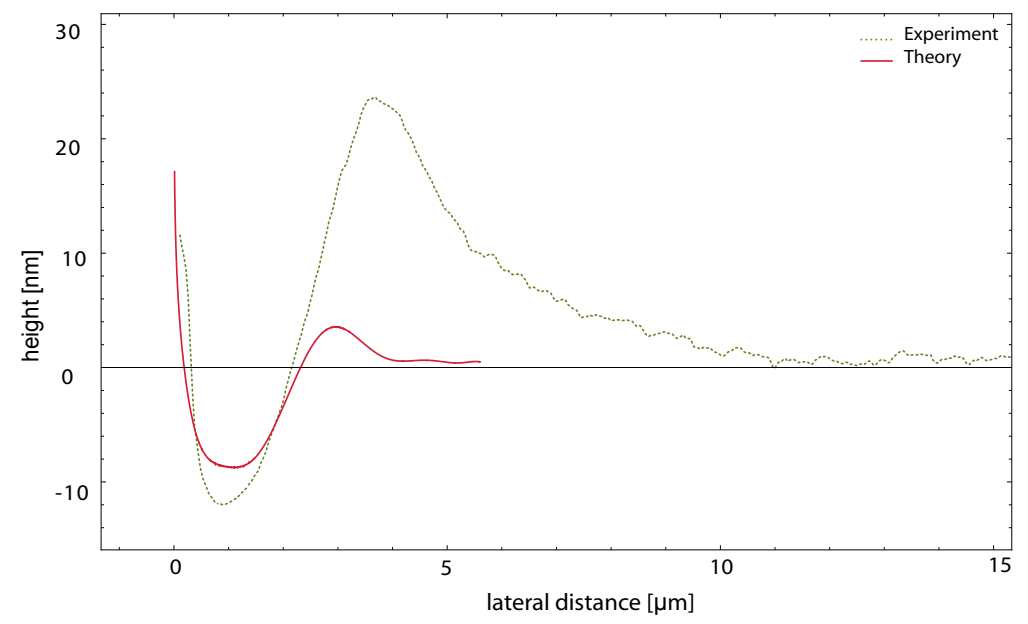

Figure 4.10: Cross section of an AMF scan of the liquid/rubber interface below a rim of PS(17.4k) dewetting on SG186 at $120^{\circ} \mathrm{C}$ (green dotted curve) compared to the deformation calculated taking into consideration only the Laplace pressure inside the liquid (red solid curve). The radius of the hole is $r=12 \mu \mathrm{m}$.

From the above, we can conclude that dynamic effects do indeed play a significant role during dewetting. If no energy loss took place at the PS/PDMS interface, then the implementation in our theoretical model of the Laplace pressure would be sufficient in order to explain the shape and scale of the deformation. The fact that it does not, makes it evident that a more complete model has to be developed that takes into consideration the tangential forces that act on the liquid/rubber interface as well as the possibility that a non-linear elasticity theory might be needed in order to better describe the substrate deformation. 



\section{Chapter 5}

\section{Dewetting on viscous liquid substrates}

We start this chapter with a brief review of the theory of dewetting on a liquid substrate. Let us consider the system shown in Figure 5.1, where A and B are in the liquid phase. The wetting behavior is described by the spreading parameter $S=$ $\gamma_{B}-\left(\gamma_{A}+\gamma_{A B}\right)$, where $\gamma_{A}$ and $\gamma_{B}$ are the surface tensions of the liquids $A$ and $B$ respectively and $\gamma_{A B}$ is the interfacial tension between $A$ and $B$. In order for liquid $A$ to dewet on liquid $B$, the spreading coefficient must be negative. In this case, the upper film will deform the lower film as it dewets and one may note that, at equilibrium, both the horizontal and vertical components of the capillary force must vanish at the three-phase contact line (Figure 5.1). Balancing the interfacial and surface forces yields the von Neumann construction:

$$
\begin{gathered}
\gamma_{A}=\gamma_{B} \cos \theta_{A}+\gamma_{A B} \cos \theta_{B} \\
\gamma_{B} \sin \theta_{A}=\gamma_{A B} \sin \theta_{B}
\end{gathered}
$$

which in the limit of small contact angles can be written as:

$$
\gamma_{A} \theta_{A}=\gamma_{A B} \theta_{B}
$$

where $\theta_{A}$ and $\theta_{B}$ are the contact angles defining the deformation into the upper and 


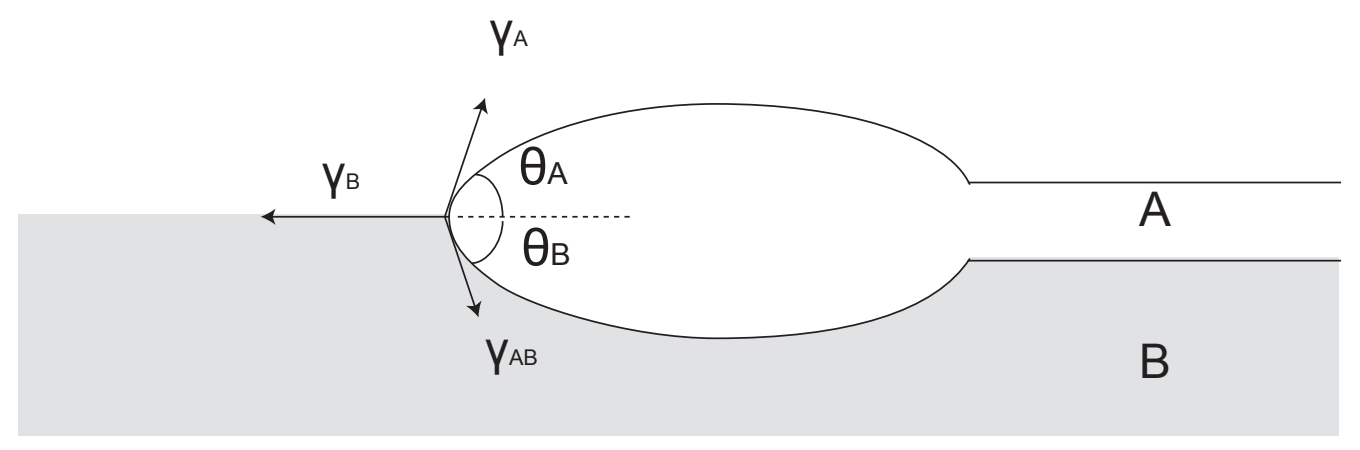

Figure 5.1: Schematic diagram of the von Neumann construction showing the rim of the dewetting film.

lower film respectively. Balancing the surface tensions horizontally and vertically in the limit of small angles leads to $S=-1 / 2 \gamma \theta_{e}^{2}$ where $\gamma=\gamma_{A} \gamma_{B} /\left(\gamma_{A}+\gamma_{B}\right)$ is the effective surface tension and $\theta_{e}=\theta_{A}+\theta_{B}$.

Some years ago, Brochard-Wyart et al. presented a detailed theoretical study [76], suggesting that liquid-liquid dewetting should exhibit a variety of different regimes, depending mainly on the relative viscosities of the two liquids, the thicknesses of the respective liquid layers and the surface and interfacial tensions involved.

According to their theory, if the viscosity of the dewetting liquid $\eta_{A}$ is much smaller than the viscosity of the substrate liquid $\eta_{B}\left(\eta_{A}<<\eta_{B}\right)$, the lower layer behaves like a solid. In this solid-like case two regimes are found: an inertial regime, where A has a low viscosity and a large contact angle and a viscous regime, where the liquid $\mathrm{A}$ is highly viscous and has a small contact angle on the lower liquid. In the latter case, the dewetting velocity is predicted to be constant and independent of the viscosity of the liquid substrate and given by:

$$
v=\frac{\gamma_{L V} \theta_{e}^{3}}{12 \ln \sqrt{2} \eta_{A}}
$$

Equation 5.4 is valid if the width of the rim is smaller than the diameter of the hole.

The opposite case of the liquid-like substrate regime occurs when $\eta_{A} / \theta_{e}>\eta_{B}$. Here three regimes are found. A purely inertial regime, a viscoinertial regime and a viscous regime similar to the one discussed above. The latter can be divided into two subregimes based on whether there is slippage at the interface or not. In the case of no slip boundary conditions, the dewetting velocity is again constant, but, in contrast to the solid-like 
situation, $v$ now depends on the substrate viscosity rather than on the viscosity of the dewetting film:

$$
v=\frac{\gamma_{L V} \theta_{e}^{2}}{12 \eta_{B}}
$$

Regarding the experimental verification of the above theory, Martin et al. [77] reported experiments on the dewetting of macroscopic liquid PDMS films on (liquid) fluorinated PDMS lower layers. They observed a constant dewetting velocity, which depends on the viscosity of the substrate and decreases with increasing substrate viscosity, in accordance with the viscous regime of the liquid-like case.

Around the same time, Lambooy et al. [78] reported a qualitative study of the dewetting of PS films on PMMA as a function of PMMA molecular weight. They observed two regimes for the dewetting velocities: at low PMMA molecular weights, the dewetting velocity decreased rapidly with increasing lower layer viscosity until it reached a minimum and thereafter it increased slowly. The authors attributed this behaviour to the change in the relative viscosities, but no experimental evidence was provided in order to prove this assumption. Later on, Qu et al. [79] continued along the same line with a series of experiments varying systematically the molecular weights of both the PS and the PMMA. In all cases the chosen molecular chain lengths were well above the entanglement length of PS and PMMA. Their results show good agreement between experiments and the theoretical predictions from Brochard-Wyart for dewetting from solid-like and liquid-like substrate layers.

In the following sections, we apply the above discussed theory of Brochard-Wyart in our system. We then extend the study of PS dewetting on PMMA of molecular weight below the entanglement length and develop a numerical model to describe the dewetting dynamics and statics for this system.

\subsection{Dewetting dynamics}

\subsubsection{Dewetting rates}

In the case of dewetting on a viscous substrate, the liquid/liquid interface is not 
constrained to a plane as it would be if the dewetting took place on a rigid solid substrate, but adjusts itself to minimize the surface free energy and the vertical component of the liquid surface tension $\gamma_{L V} \sin \vartheta_{e}$ should lead to a local deformation of the substrate close to the three-phase contact line as it is sketched in Figure 5.2 .

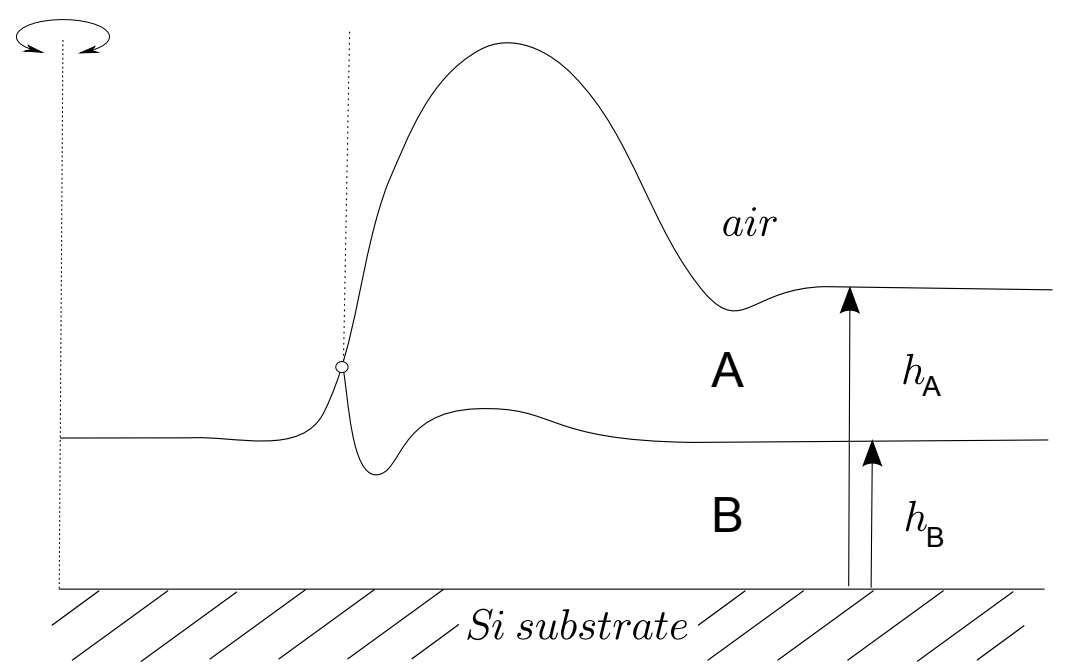

Figure 5.2: Dewetting of a liquid film A on a liquid substrate B on top of a silicon substrate. By $h_{A}$ and $h_{B}$ we denote the (relative) positions of the interfaces above the substrate surface.

We start the description of our experiments with a $100 \mathrm{~nm}$ thick PS(100k) film dewetting on liquid PMMA substrates of different chain lengths, all above the entanglement length of PMMA, which is around PMMA(10k). In order to induce dewetting, the sample PS/PMMA was annealed above the glass transition temperature $T_{g}$ of PS. After a few minutes of annealing, circular holes appear on the PS film and grow with time. The analysis of the experimental data of the hole growth as a function of time is plotted in Figure 5.3. (a). The radii of the holes grow at constant speed. We observe that the dewetting rates decrease as the substrate molecular weight, respectively the viscosity, increases for molecular weights of PMMA (62k) to (110k), whereas for molecular weights above $(110 \mathrm{k})$ the dewetting rates seem to remain almost constant.

If we keep the same set of substrates, but we change the dewetting film to the less 

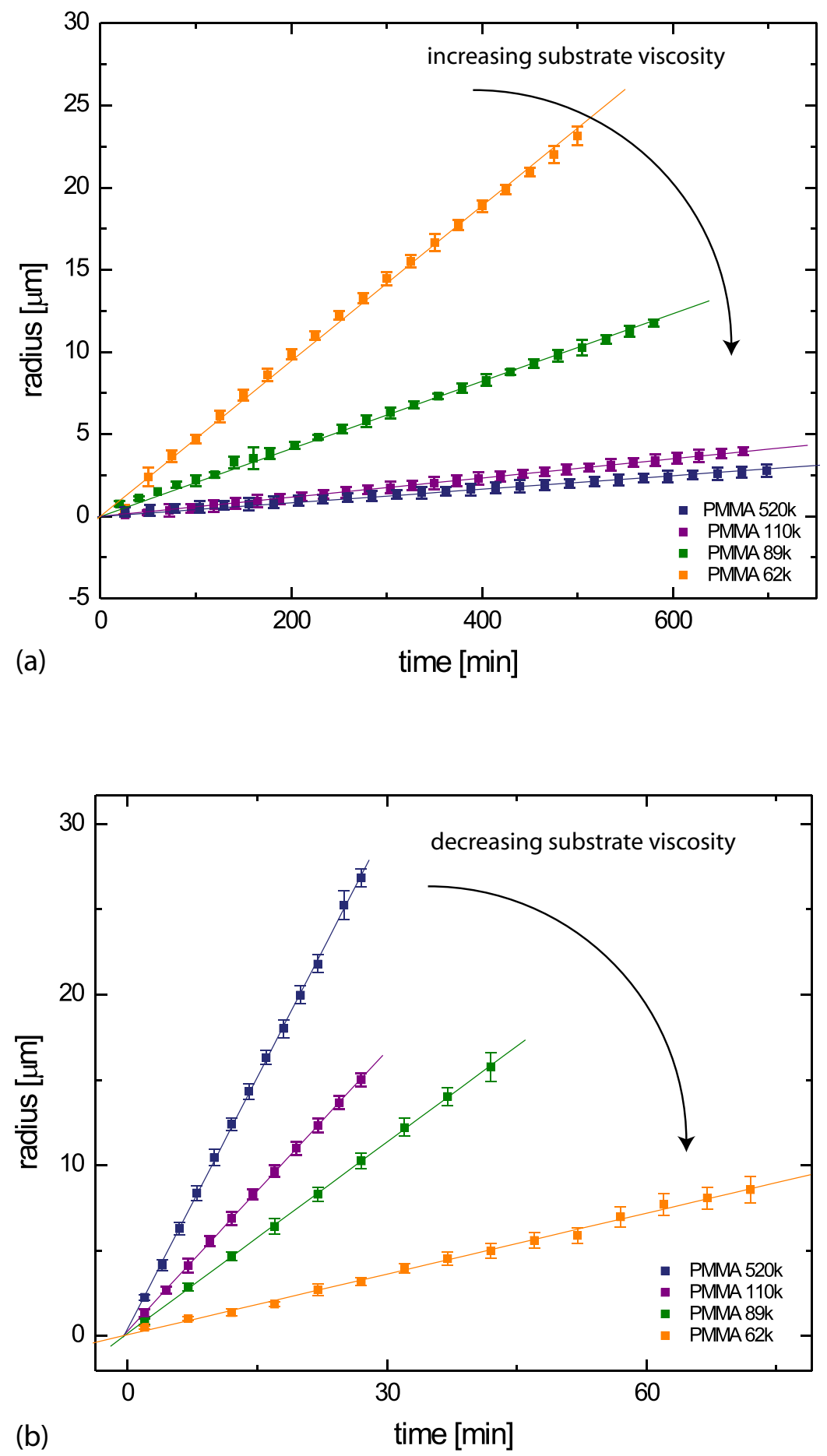

Figure 5.3: Dewetting rates of (a) a PS(100k) film and (b) a PS(17.4k) film dewetting at $160^{\circ} \mathrm{C}$ on PMMA substrates of different molecular weights. 
viscous $\mathrm{PS}(17.4 \mathrm{k})$, the dewetting velocity as a function of time exhibits the opposite behaviour: in this case, shown in Figure 5.3.(b), the dewetting rates decrease with the decreasing of the substrate molecular weight.

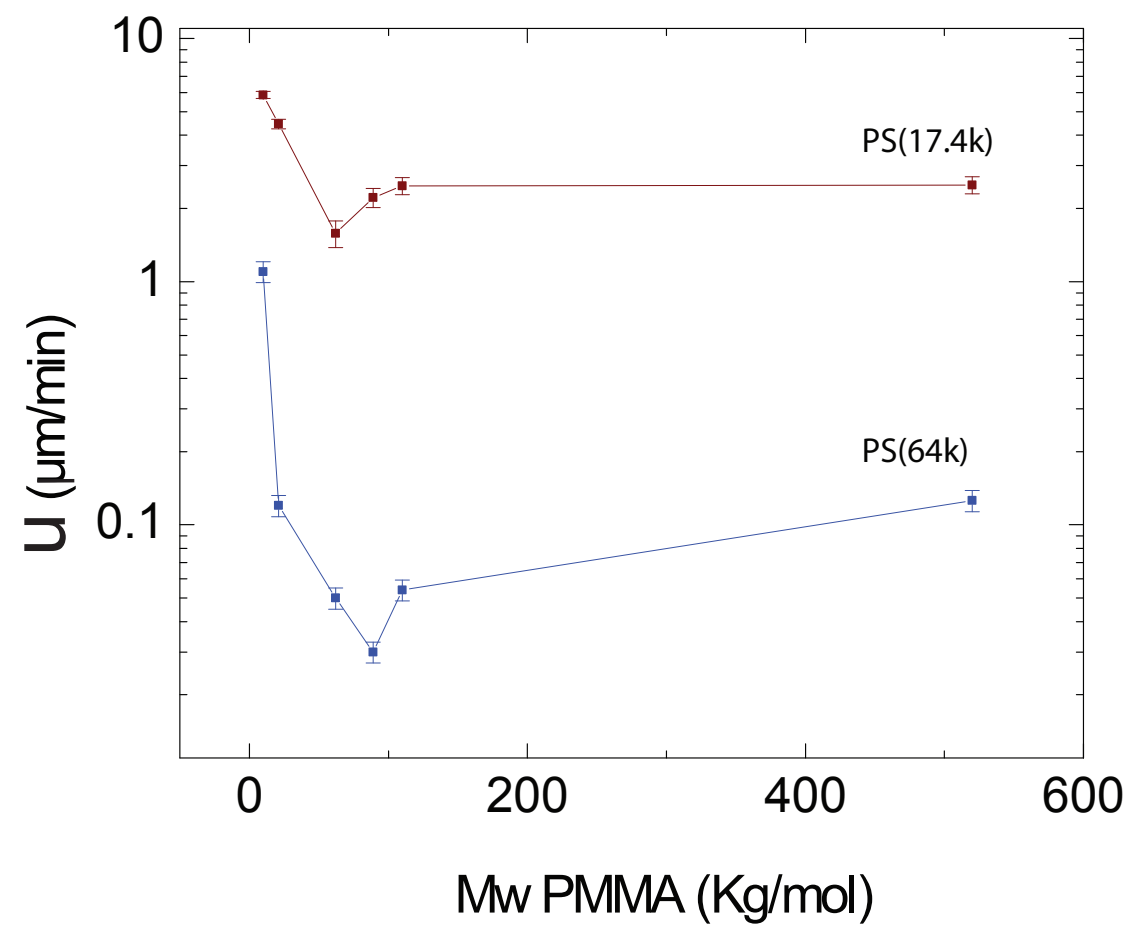

Figure 5.4: Dewetting velocities as a function of substrate molecular weight for $50 \mathrm{~nm}$ thick PS films dewetting on PMMA at $160^{\circ} \mathrm{C}$. The solid lines are guides to the eye.

To obtain a clearer idea about how the holes grow as a function of time and substrate viscosity, we have plotted on a semilogarithmic scale the dewetting velocity for two different PS films (PS $(17.4 \mathrm{k})$ and PS(64k)) as a function of substrate molecular weight shown in Figure 5.4. In this case we have used a range of molecular weights from PMMA(10k) to PMMA(520k). What we observe for both PS chain lengths is the following: for substrates of low viscosity, we recognize a regime where the dewetting velocity decreases quickly with increasing substrate viscosity until it reaches a minimum. The higher the molecular weight of the dewetting film, the higher the substrate molecular weight that corresponds to the minimum velocity. After which, the dewetting velocity starts to increase slowly with increasing substrate molecular weight, respectively viscos- 
ity, until the substrate viscosity does not play a role anymore and the dewetting velocity stays constant.

The regime where the dewetting velocity decreases with increasing molecular weight can be explained by the viscous regime of the liquid-like case, where the dewetting velocity is constant with time and inversely proportional to the substrate viscosity (Equation 5.5). Additionally, the regime where the dewetting velocity remains constant as a function of the substrate molecular weight can be explained by the viscous regime of the solid-like case as we discussed previously in the theory, where the dewetting velocity is inversely proportional to the viscosity of the dewetting film (Equation 5.4). Since the viscosity of the dewetting film remains constant, so does the dewetting velocity. Regarding the intermediate regime, where the dewetting velocity increases with increasing molecular weight, it can be thought of as a transition regime between the liquid-like and the solid-like regime. Nevertheless, it has not been predicted by the theory of Brochard-Wyart.

For a better understanding of the comparison with the theoretical predictions, it needs to be emphasized that the shape of the rims derived experimentally, as we will see in the next sections, differs significantly from the von Neumann construction presented at the beginning of this chapter, on which the theory of Brochard-Wyart is based. Moreover, the substrates of our experimental system do not clearly fall neither in the bulky case nor in the thin film case considered by the theory. They rather belong to a transition region between the two. Therefore, the aforementioned theory does not fully correspond to our system. Thus it cannot explain in detail its dewetting dynamics and a new model is needed that takes into account the specific characteristics of our system.

\subsubsection{Rim profile}

To understand the rim growth dynamics it is essential that we also take into account the shape of the rim at the air/PS interface, as well as the deformation of the PS/PMMA interface underneath the rim. Once the holes had reached the desired radius, we immediately quenched our samples to room temperature in order to bring the polystyrene film to the glassy state and imaged the shape of the dewetting PS rim by AFM. Regarding the imaging of the deformation of the PS/PMMA interface, since PMMA is glassy at room temperature, it is sufficient to remove the overlying PS film and image directly 
the deformation. To do that, we immerse the sample into a selective solvent (Cyclohexane) for ca. $1-2$ minutes, which removes the PS and, after drying it, we subsequently image the remaining PMMA film, which, in room temperature, is glassy and thus has maintained its deformation.
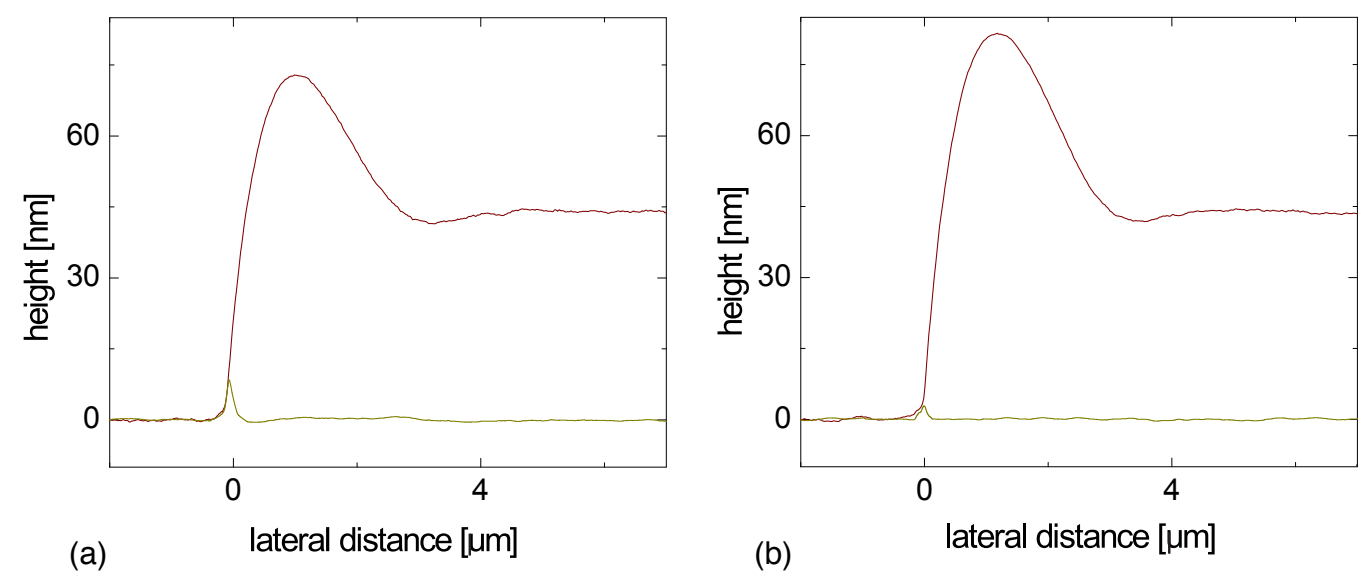

Figure 5.5: Cross-sections of AFM scans of the rim and underlying substrate deformation of a $\mathrm{PS}(17.4 \mathrm{k})$ film dewetting at $160^{\circ} \mathrm{C}$ on a (a) $\mathrm{PMMA}(62 \mathrm{k})$ substrate and (b) PMMA(520k) substrate.

In Figure 5.5 we compare the rim and corresponding deformations of a $\operatorname{PS}(17.4 \mathrm{k})$ film on PMMA(62k) and on PMMA(520k). The radius of the hole of the two systems is similar, the film thickness and the dewetting temperature are kept constant in both cases. We observe that the rim presents a damped oscillatory behaviour towards the undisturbed part, similar to the one found for the PS film dewetting on the softest elastic substrate discussed in the previous chapter. The deformation of the underlying substrate is limited mainly close to the three-phase contact line, where the surface tension seems to be lifting the substrate upwards. The shape of the deformation remains qualitatively the same in both systems, but for the less viscous substrate, it is clearly of a larger scale.

In Figure 5.6 we make a qualitative comparison of the rim and corresponding deformation of a $50 \mathrm{~nm}$ PS(17.4k) film and a $100 \mathrm{~nm} \mathrm{PS}(100 \mathrm{k})$ film dewetting at $160^{\circ} \mathrm{C}$ on a substrate of PMMA(62k). We observe that, in both systems, the deformation is again more pronounced close to the TPCL. Furthermore, in this case, the deformation of the underlying substrate is not only quantitatively different between the two systems, but 
also qualitatively. Specifically, we see that, for the higher viscosity dewetting film, after the lifting of the TPCL, there appears also an oscillation on the PS/PMMA interface, which disappears into the undisturbed PMMA substrate. Furthermore, on the same system, the PS seems to be penetrating into the PMMA substrate, forcing it to move along the direction of the growing hole, resulting in the air/PMMA interface being lower than the PS/PMMA interface.

(a)
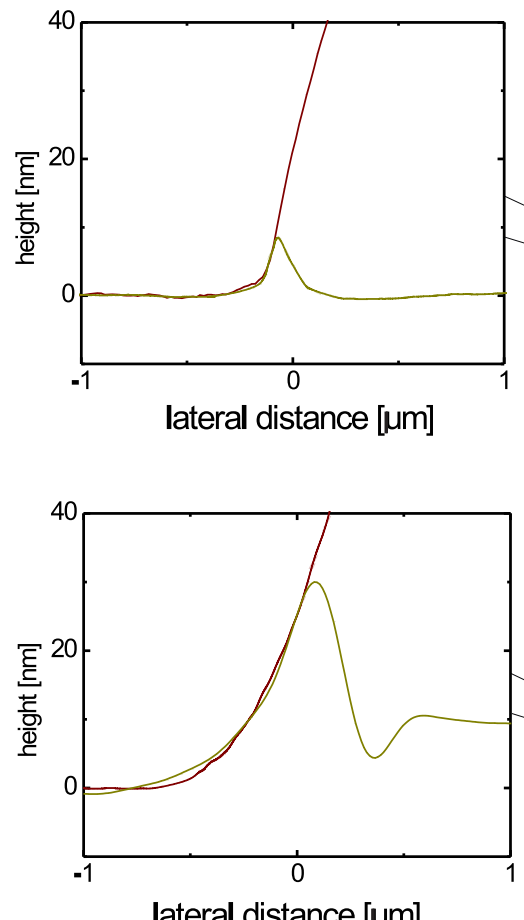

(b)

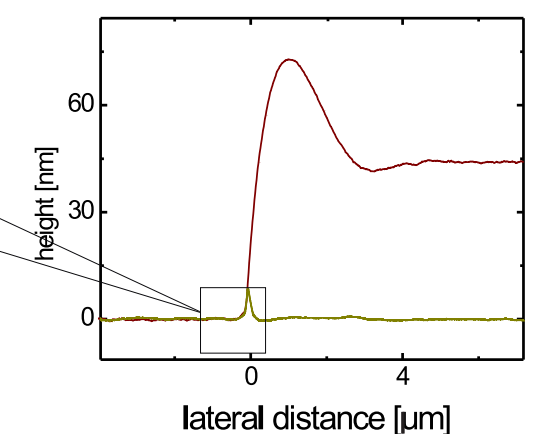

lateral distance $[\mu \mathrm{m}]$

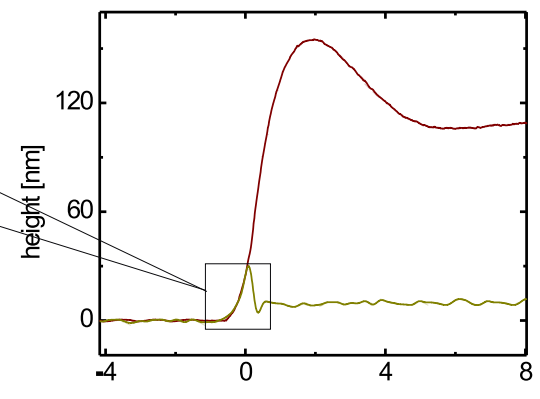

lateral distance $[\mu \mathrm{m}]$

Figure 5.6: (a) Cross-section of an AFM scan of the rim and underlying substrate deformation of a $50 \mathrm{~nm}$ thick $\mathrm{PS}(17.4 \mathrm{k})$ film dewetting at $160^{\circ} \mathrm{C}$ on $\operatorname{PMMA}(62 \mathrm{k})$. (b) Cross-section of an AFM scan of the rim and underlying substrate deformation of a $100 \mathrm{~nm}$ thick PS(100k) film dewetting at $160^{\circ} \mathrm{C}$ on PMMA(62k).

So far, we have studied the shape of the rim of dewetting PS films on PMMA substrates of molecular weight above the entanglement length. We have seen that the PS/PMMA interface deformation is localized close to the TPCL and its lateral extension is significantly smaller than the total size of the rim, as opposed to the theoretical model discussed at the beginning of this chapter, which predicts a lens-like shape. In order to exclude that any of our observations discussed above might be the result of a 
shear-rate dependent viscosity or slip conditions in any of our liquids, both mechanisms that can drastically change the dynamics of the dewetting process, from now on, we will restrict our study to Newtonian liquids, both for the dewetting film and the underlying substrate.

In Figure 5.7 are shown the rims of a growing hole of $\mathrm{PS}(17.4 \mathrm{k})$ film dewetting on $\operatorname{PMMA}(10 \mathrm{k})$ for $r_{1}=4 \mu \mathrm{m}$ and $r_{2}=17 \mu \mathrm{m}$. We observe that, as the rim grows the damped oscillatory structure also becomes more evident. In parallel the height of the lifting of the interface at the three-phase contact line seems to not depend significantly on the hole radius. The oscillatory structure of the PS/PMMA interface that we observed in the case of $\operatorname{PS}(100 \mathrm{k})$ on $\operatorname{PMMA}(62 \mathrm{k})$ is again present, but in this case the "trench" actually penetrates into the substrate. Furthermore, the deformation is not limited anymore to the vicinity of the TPCL, but it extends underneath the hole rim profile with the presence of a slight protuberance with width equal to the rim width.

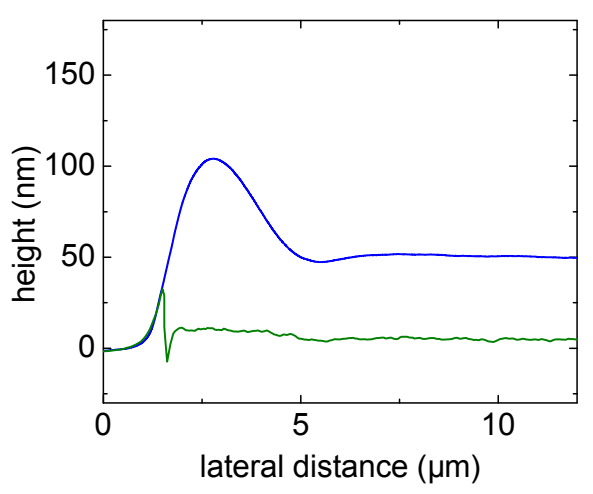

(a)

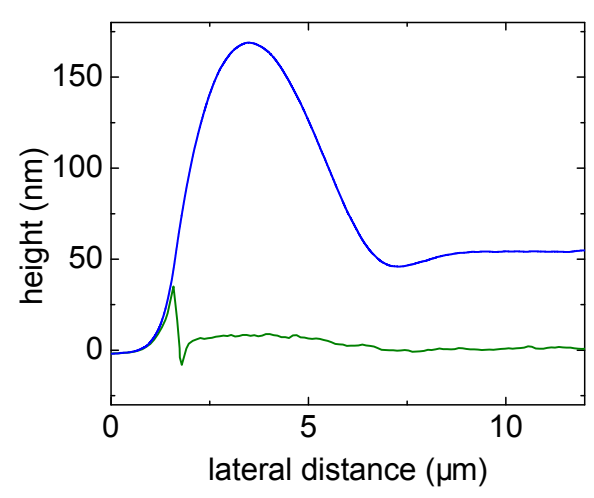

(b)

Figure 5.7: Cross-sections of AFM scans of the rim and underlying substrate deformation of a growing hole of a $\operatorname{PS}(17.4 \mathrm{k})$ film dewetting at $170^{\circ} \mathrm{C}$ on $\operatorname{PMMA}(10 \mathrm{k})$ with (a) $r_{1}=4 \mu m$ and (b) $r_{2}=17 \mu m$.

In Figure 5.8 we compare the rims of a $\mathrm{PS}(17.4 \mathrm{k})$ film on PMMA substrates of different chain lengths: $\operatorname{PMMA}(4 \mathrm{k})$ and $\operatorname{PMMA}(10 \mathrm{k})$. The air/PS interface of the rim seems to hardly depend on the PMMA chain length. On the other hand, we observe a strong dependence of the PS/PMMA interface deformation on the PMMA chain length. The general features of the deformation at the liquid/liquid interface are the lifting of 


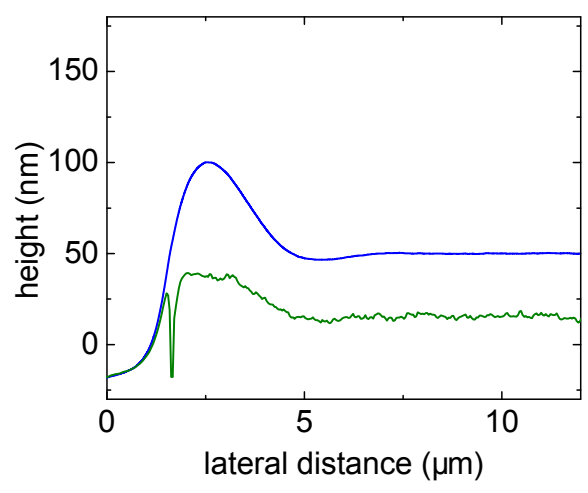

(a)

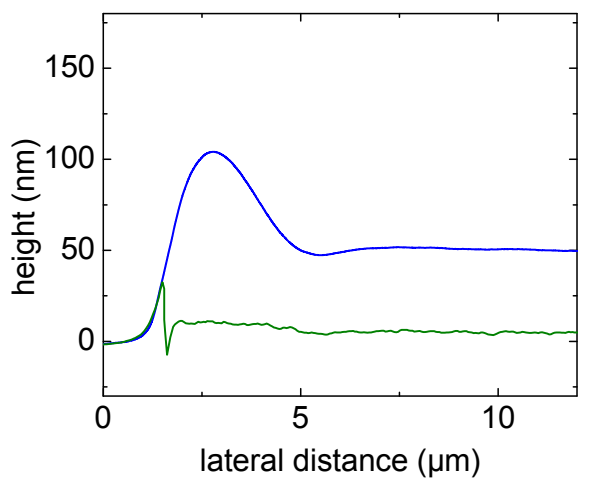

(b)

Figure 5.8: Cross-sections of AFM scans of the rim and underlying substrate deformation of a $4 \mu \mathrm{m}$ hole of a $\operatorname{PS}(17.4 \mathrm{k})$ film dewetting at $170^{\circ} \mathrm{C}$ on a (a) $\operatorname{PMMA}(4 \mathrm{k})$ substrate and (b) PMMA(10k) substrate.

the interface at the three-phase contact line and the "trench" right behind it in direction of the unperturbed PS film. Furthermore, towards the unperturbed film we find a wide rim of the liquid/liquid interface followed by a trough whose shape resembles the shape of the air/PS interface above it. For increasing molecular weight of the PMMA substrate and thus for increasing viscosity, the amplitude of the deformation of the liquid/liquid interface decreases, i.e. for PMMA (10k), the lifting of the three-phase contact line and the trench are still well visible, whereas the deformation of the liquid/liquid interface that follows the shape of the PS rim tends to disappear.

The AFM scans of the PS rims for decreasing PMMA molecular weight (Figure 5.5. Figure 5.7 and Figure 5.8 indicate that we establish a transition from a solid-like to a liquid-like behaviour of the substrate, similar to the one observed in the graph of the dewetting velocities in Figure 5.4 .

\subsubsection{Comparison with numerical simulations}

In order to explain theoretically our results, we have compared them with simulations based on a theoretical model developed by A. Münch, B. Wagner and D. Peschka in collaboration with our group. The details of the theoretical calculations can be found 
in Appendix C.

In the following, the numerically obtained dewetting rates and rim profiles are compared with the corresponding experimental results. We note that our numerical simulations of the dewetting rates shown in Figure 5.9.(b) are qualitatively consistent with the experimentally observed trend (Figure 5.9.(a)) for PS and PMMA below the entanglement length, in which the dewetting rates decrease with increasing substrate viscosity. Starting at very small hole radii we find an increasing dewetting velocity within the first
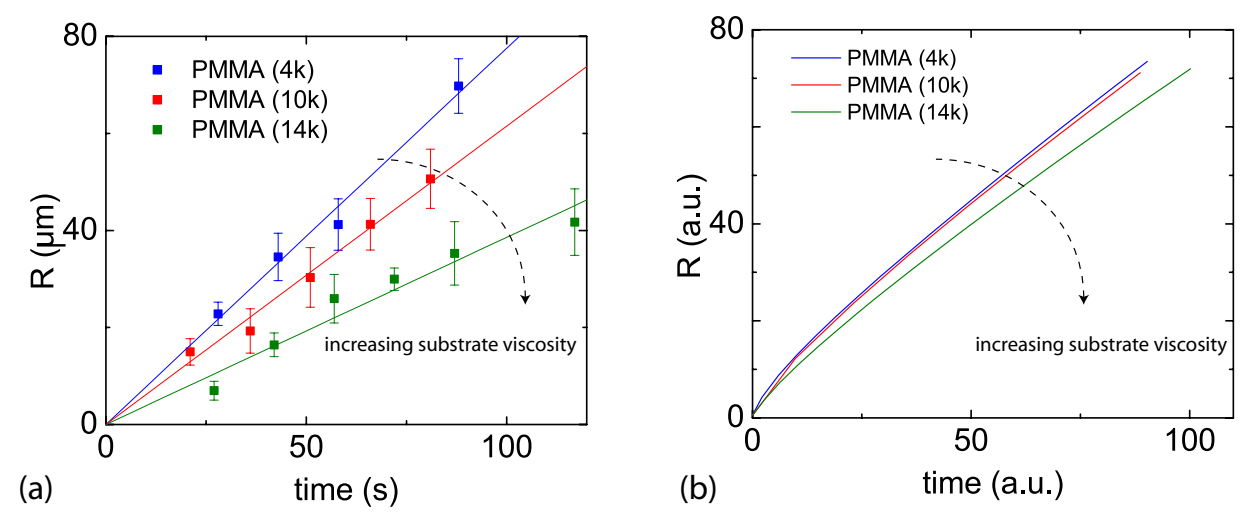

Figure 5.9: (a) Experimental results and (b) simulations for the dewetting rates of a $50 \mathrm{~nm}$ PS $(17.4 \mathrm{k})$ film dewetting at $170^{\circ} \mathrm{C}$ on PMMA substrates of different molecular weights.

micrometers, whereas, for larger hole radii the velocity settles as constant $(r \sim t)$. The non-linear increase of the dewetting velocity as a function of time is not visible in the experimental results due to the limitations in the resolution for very small hole radii.

The numerically obtained rim profiles are displayed in Figure 5.10.(b) in comparison with the corresponding experimental results (Figure 5.10.(a)). The basic features of the PS rim shape and the deformation of the liquid/liquid interface are also reflected in good agreement by our numerical results. Any deviations between theory and experiment can be safely attributed to the uncertainty in the input parameters used for the numerical calculations. The sensitivity of the system on experimental parameters can be seen in Figure 5.11. Here are shown the rims and corresponding deformations obtained changing only one parameter each time. Specifically, between Figure 5.11. (a) and Figure 5.11. (b), the relative surface tension $\gamma$ is kept fixed, while the relative viscosity $\alpha$ is changed. Whereas, between Figure 5.11.(b) and Figure 5.11.(c), the relative viscosity is kept fixed, 

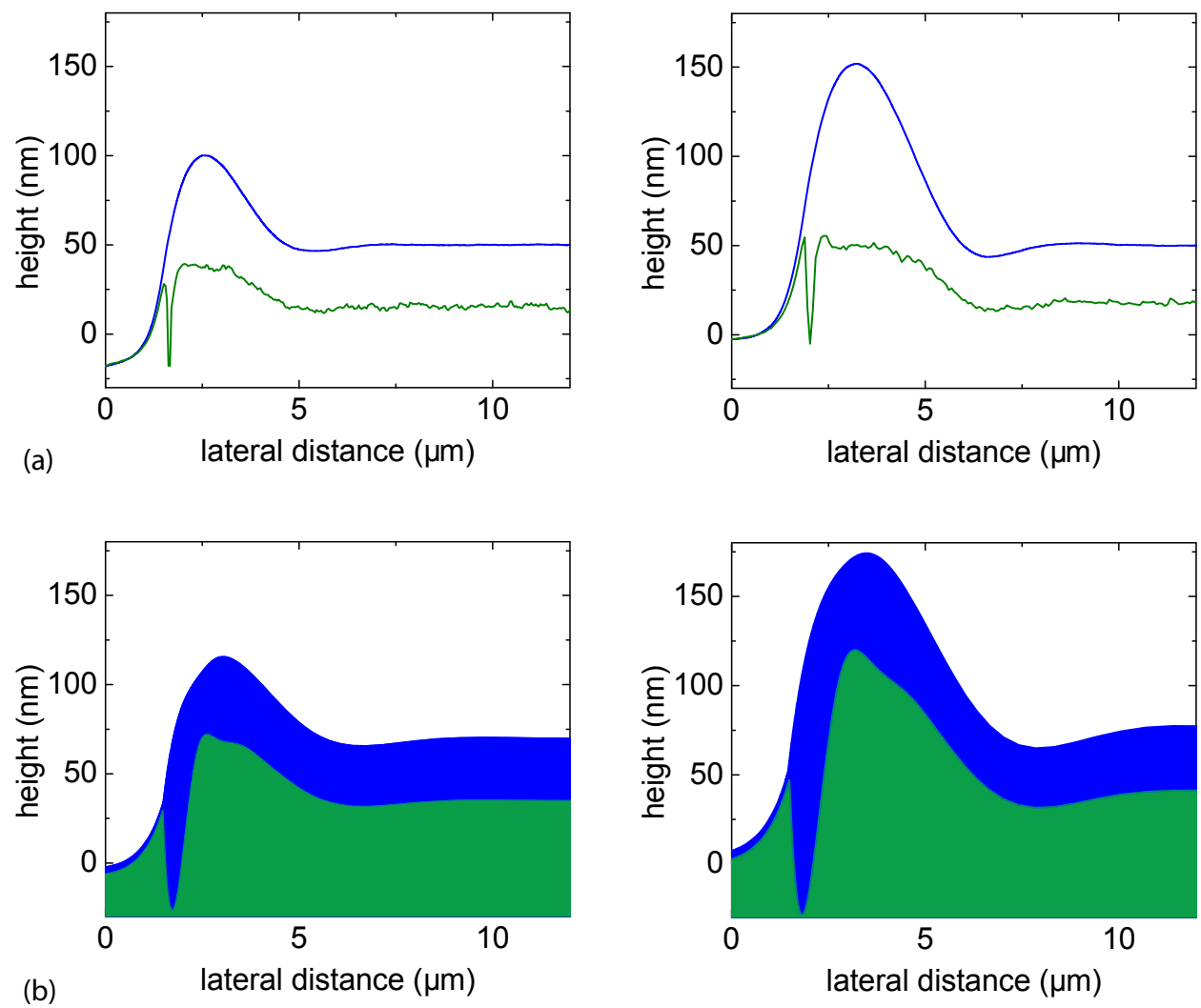

Figure 5.10: (a) Experimental results of the rim and interface deformation of a growing hole $\left(r_{1}=4 \mu \mathrm{m}\right.$ and $\left.r_{2}=10 \mu \mathrm{m}\right)$ of a PS (17.4k) film dewetting at $170{ }^{\circ} \mathrm{C}$ on PMMA (4k). (b) Solutions of Equation C.6 of the rim and interface deformation of a PS (17.4k) film dewetting at $170^{\circ} \mathrm{C}$ on a PMMA (4k) substrate for the same hole radii. The simulation parameters are $\alpha=2.3, \gamma=0.2, h *=0.02 H, \phi *=4.33, H=200 \mathrm{~nm}$ and initial data are $h_{1}=H$ and $h_{2}$ is a smooth step function with values $H+h *$ for $R<0.3 \mu m$ and $H+35 \mathrm{~nm}$ for $R>0.5 \mu \mathrm{m}$.

while the relative surface tension is changed. All other parameters, such as contact angle, film thickness etc. are held fixed. From the comparison between these three numerically obtained rims, it is evident that, changing even only one parameter each time, the effect this has on the resulting rim is impressive.

Therefore, considering that many of the parameters on which our simulations are based are given with a significant uncertainty, we can conclude that our numerical model can accurately describe the liquid/liquid dewetting. 

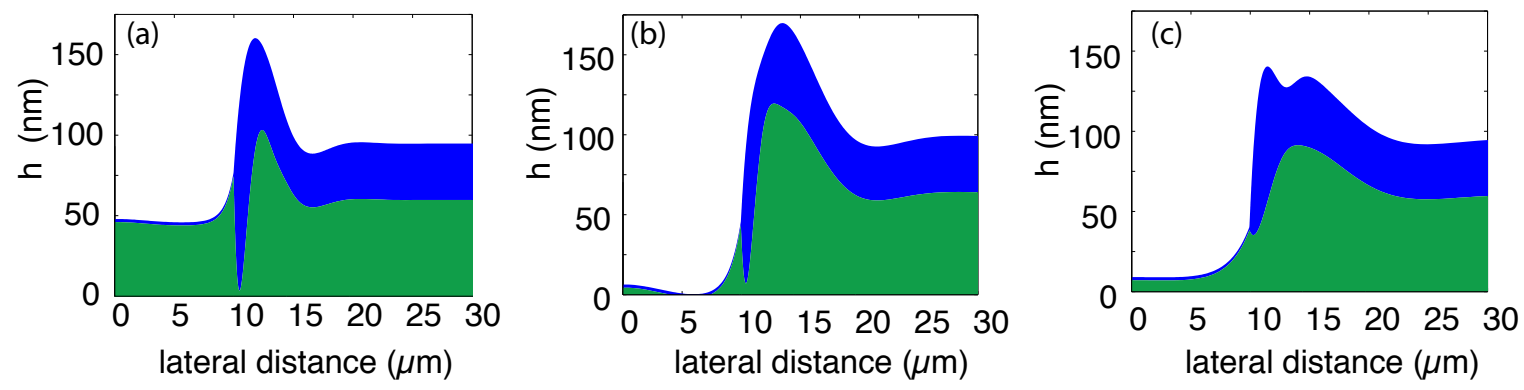

Figure 5.11: Solutions of Equation C.6 of the rim and interface deformation of a PS (17.4k) film dewetting at $170^{\circ} \mathrm{C}$ on a PMMA (4k) substrate for the same hole radii. The varied simulation parameters are: (a) $\alpha=23, \gamma=0.4$, (b) $\alpha=0.23, \gamma=0.4$ and (c) $\alpha=0.23, \gamma=4.0$.

\subsubsection{Late stage dewetting}

As for the PS droplets on the rubber elastic substrates, sessile nanodroplets on viscous substrates could also give valuable information regarding the viscous properties not only of the substrate, but also of the dewetting film itself. A detailed study of the shape of the air/PS and PS/PMMA interface of the droplets in equilibrium was not possible, as the time required to reach equilibrium at the temperatures employed in our experimental setup exceeded by far the typical experimental time scales. In Figure 5.12, is depicted a 3d AFM image of a PS droplet on PMMA and the corresponding underlying deformation after 24 hours of annealing at $170^{\circ} \mathrm{C}$. From the shape of the deformation it is clear that the system has not yet reached equilibrium, as one would expect a lens-like deformation due to the Laplace pressure inside the liquid rather than the upwards deformed substrate underneath the drop. We compare the shape of this droplet with the time series shown in Figure 5.13 calculated from our simulations based on the model described in Appendix C. The relative time lapse between each image is given, with $t$ being in the order of magnitude of $1-10$ minutes. Based on that, it is easy to calculate that the droplet in Figure 5.13. (d) is obtained after at least one year of annealing at $170^{\circ} \mathrm{C}$. Thus, a droplet needs several years of annealing under the specific experimental conditions in order to reach equilibrium. 


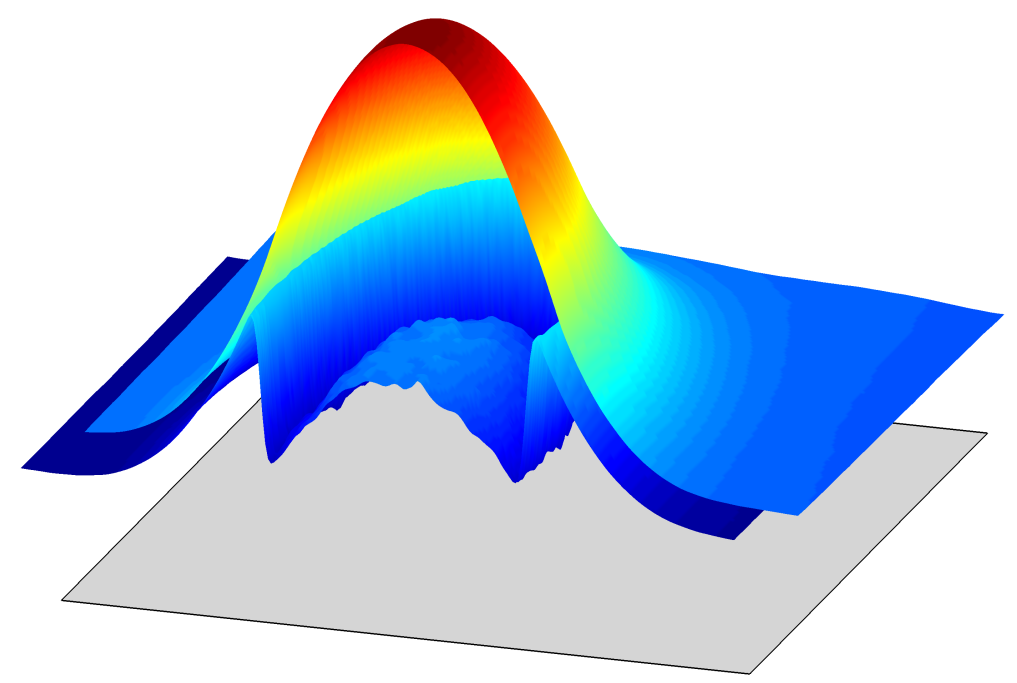

Figure 5.12: 3d AFM image of a PS(17.4k) droplet on PMMA(4k) after 24 hours of annealing at $170^{\circ} \mathrm{C}$. The droplet has not reached equilibrium yet.

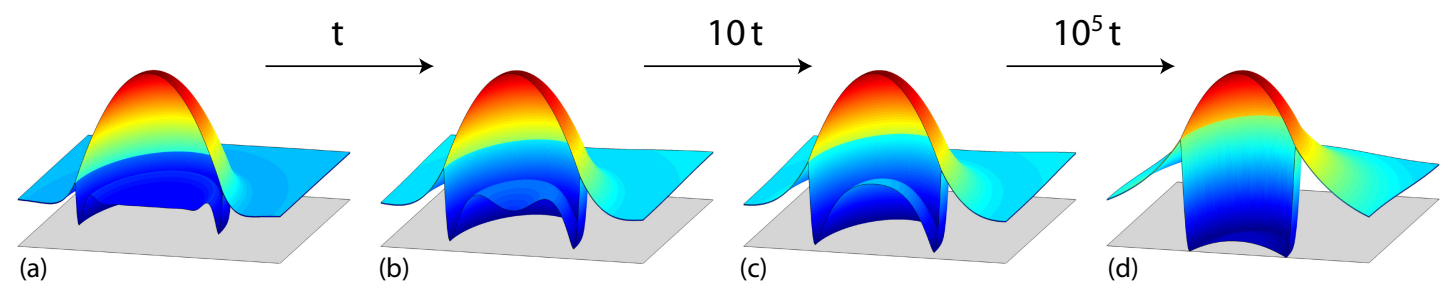

Figure 5.13: Time series of droplets of PS(17.4k) on PMMA(4k) obtained from numerical simulations. 



\section{Chapter 6}

\section{Wetting phenomena on topographically structured rubber elastic substrates}

The control and growth of surface liquid morphologies on solid surfaces has a variety of applications in fields such as microelectronics, catalysis and lithography. Generally, there are two approaches regarding the manipulation of liquids in open microfluidic structures. The first one is to prepare patterns with different wettability on planar substrates [80 85]. A major disadvantage of this approach is that the Laplace pressure of any liquid morphology on a planar surface will be strictly positive and a liquid will never spread spontaneously along the chemical patterns when brought in contact with a large liquid reservoir of zero Laplace pressure. The second approach is to offer an appropriate surface topography to the liquid [36, 39, 86]. This exploits the fact that the liquid prefers to wet wedges and grooves rather than planar surfaces, provided that its contact angle on the substrate is sufficiently small. Depending on control parameters such as liquid volume, contact angle and substrate geometry, a rich variety of liquid morphologies can be found on topographically structured substrates.

In this chapter, we will first present the state of the art regarding liquid morphologies on rigid topographically structured substrates, followed by some preliminary results of our study on wetting morphologies on rubber elastic rectangular grooves. These results are based on experiments performed by Dominik Michler under the supervision of the 
author of this thesis, in the course of a lab-training program.

\subsection{Wetting morphologies on rigid topographically structured substrates}

\subsubsection{Triangular grooves}

Khare et al. [37] carried out an experimental and theoretical study on the wetting of liquid morphologies in triangular grooves that were symmetric along their long axis and that were characterised either by their opening angle $\alpha$ or by their wedge angle $\psi$, given by $\psi=\frac{1}{2}(\pi-\alpha)$ as shown in Figure 6.1. The experiments consisted in evaporating polystyrene (PS) on substrates of different wedge angles and wettabilities and imaging and classifying the resulting liquid morphologies.
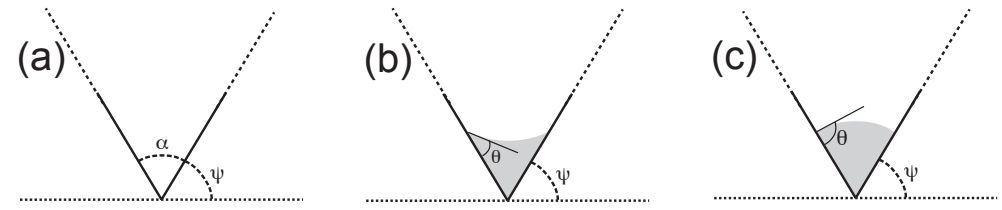

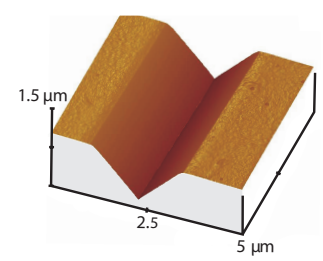

$\psi=54.7^{\circ}$

(d)

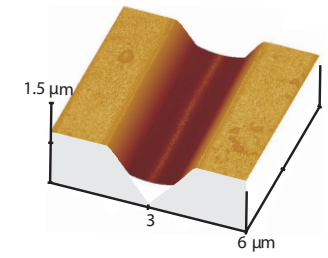

$\psi=54.7^{\circ}, \theta=27 \pm 1^{\circ}$

(e)

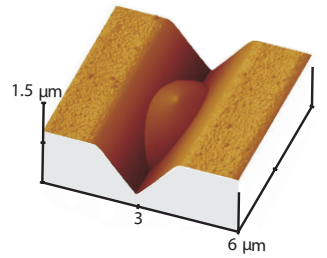

$\psi=54.7^{\circ}, \theta=64 \pm 1^{\circ}$

(f)

Figure 6.1: Sketch of infinite wedges with wedge angle $\psi$ (a), including a wetting morphology for small liquid volume and low contact angle $\theta<\psi(b)$, including a wetting morphology with high contact angle $\theta>\psi(\mathrm{c})$, and corresponding AFM images (d, e, f). (Image courtesy of K. Khare.) 
Depending on the volume of the liquid and its contact angle on the substrate, one can obtain different wetting morphologies in the triangular groove. If the liquid volume deposited in the groove is small enough such that the three phase contact line does not "feel" the ridge, the depth of the channel can be considered infinite and the wetting morphologies obtained are either the wedge wetting morphology [W] for $\theta<(\pi / 2-\alpha / 2)$ (Figure 6.1.(b) and (e)) or the corner drop morphology [cD] for $\theta>(\pi / 2-\alpha / 2)$ (Figure 6.1. (c) and (f)).

Increasing the volume such that the three phase contact line gets pinned at the edge formed by the wall of the groove and the ridge, three morphologies can appear as shown in Figure 6.2. For small contact angles, a liquid forms a pinned wedge [pW], i.e. a filament with negative Laplace pressure $\left[F^{-}\right]$that extends to the entire length of the groove (Figure 6.2. (a) and (d)). For intermediate contact angles the liquid forms filaments of finite length with positive Laplace pressure $\left[\mathrm{F}^{+}\right]$. Finally, for large contact angles, the liquid forms droplets [D] that are overspilling on the ridges (Figure 6.2.(c) and (f)).

(a)
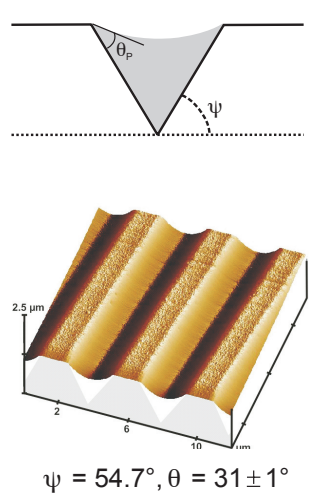

(d) (b)
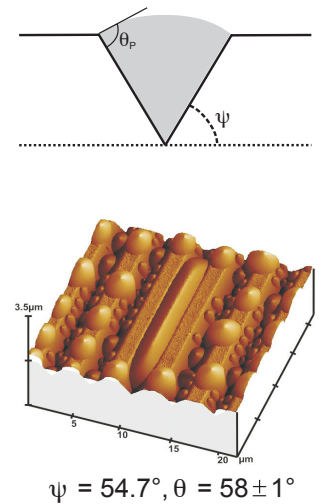

(e)
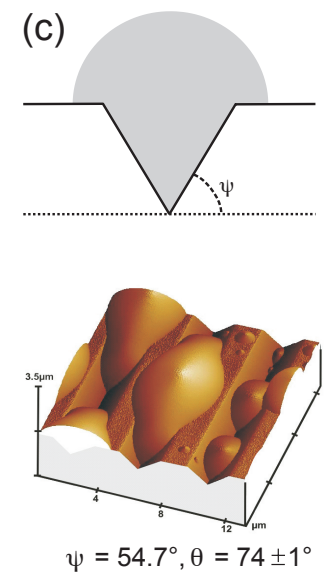

(f)

Figure 6.2: Wetting morphologies in a triangular groove. (a), (b) and (c) show sketches of morphologies for increasing contact angle. Corresponding AFM micrographs are shown in $(\mathrm{d}),(\mathrm{e})$ and (f). (Image courtesy of K. Khare.)

Based on their results, Khare et al. [37] derived a morphology diagram for triangular grooves (Figure 6.3 that depends only on the wedge angle $\psi$ of the groove and on the contact angle $\theta$ of the liquid on the substrate. This diagram has been derived via a 


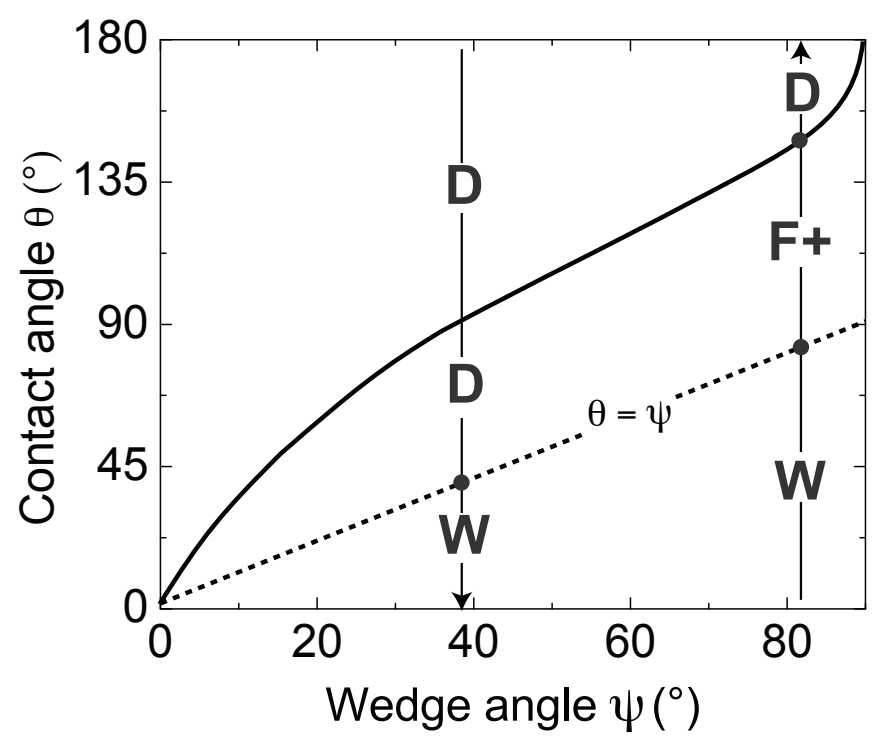

Figure 6.3: Morphology diagram for a liquid with contact angle $\theta<90^{\circ}$ in a triangular groove of wedge angle $\psi$.

simple calculation based on the stability of the liquid filament morphology. There are three regions in the diagram representing three different wetting morphologies. The bottom region corresponds to the wedge wetting [W], where liquid filaments with negative Laplace pressure extend along the entire length of the groove. The top region corresponds to the drop morphology $[\mathrm{D}]$, whereas the middle region is a metastable region, where coexistence of two morphologies, i.e. $\left[F^{+}\right]$and $[\mathrm{D}]$ can be found, depending on the previous morphology of the liquid: starting from the top region with a drop morphology and moving downwards in the diagram, i.e. decreasing the contact angle, one gets wedge wetting morphologies only after crossing the dotted line, i.e. at $\theta=\psi$. In this case, in the middle region, one observes drop morphologies. On the other hand, if one starts with a liquid wedge $[\mathrm{W}]$ and moves upwards in the diagram, i.e. increasing the contact angle, then, as soon as the dotted line has been crossed, filaments with positive Laplace pressure $\left[F^{+}\right]$appear and the drop morphology $[\mathrm{D}]$ is generated only after having crossed the black line and having entered the top region. From Figure 6.3 it is clear that, in a triangular groove, different morphologies can be obtained by simply changing the contact angle of the liquid. A similar behaviour would emerge if one changed the wedge angle $\psi$. 


\subsubsection{Rectangular grooves}

Adding one more corner to the triangular groove and keeping $\psi=90^{\circ}$ results in obtaining a rectangular groove. Seemann et al. [36] investigated experimentally and theoretically the wetting on rectangular microstructured surfaces and derived a morphology diagram for rectangular grooves that depends only on the aspect ratio $X$ of the grooves, i.e. the ratio of the height to the width of the groove and on the contact angle $\theta$ of the liquid on the substrate. A summary of their experimental results can be seen in Figure 6.4. where the variation of the contact angle of the liquid on the substrate and of the groove aspect ratio, leads to the formation of different liquid morphologies. In

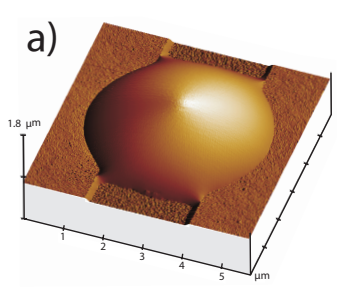

$X=0.031, \theta=43 \pm 1^{\circ}$

d)

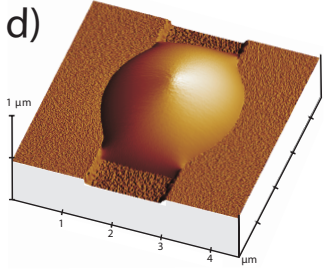

$X=0.063, \theta=54 \pm 1^{\circ}$

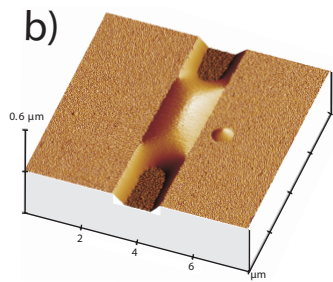

$X=0.063, \theta=20 \pm 2^{\circ}$

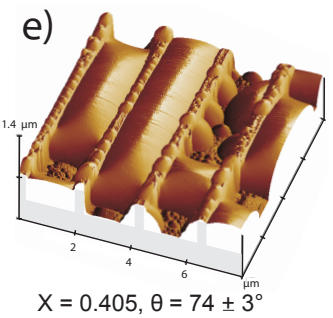

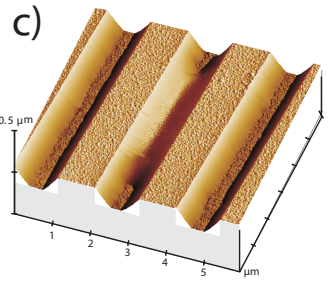

$X=0.165, \theta=20 \pm 3^{\circ}$

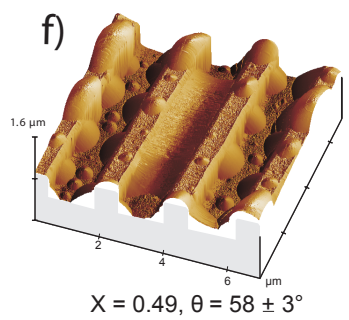

Figure 6.4: AFM images of liquid morphologies in rectangular grooves. For $\theta<45^{\circ}$ (top row) one can observe (a) droplets connected to a pinned wedge $[\mathrm{D} / \mathrm{pW}]$, (b) extended filaments with positive Laplace pressure connected to a pinned wedge $\left[F^{+} / \mathrm{pW}\right]$ and (c) extended filaments with negative Laplace pressure connected to a pinned wedge $\left[F^{-} / \mathrm{pW}\right]$. For $\theta>45^{\circ}$ (bottom row), one can observe the same basic morphologies without the thin liquid wedges $[\mathrm{W}]$ in the groove corners, i.e. (d) overspilling droplets [D] that spread on the ridges, (e) extended filaments with positive Laplace pressure $\left[\mathrm{F}^{+}\right]$ and (f) extended filaments with negative Laplace pressure $\left[F^{-}\right]$. (Image courtesy of R. Seemann.)

the figure, the top row corresponds to a contact angle of $\theta<45^{\circ}$ and aspect ratios $X$ that range from 0.03 to 0.17 , whereas the bottom row corresponds to a contact angle of 
$\theta>45^{\circ}$ and aspect ratios $X$ that range from 0.06 to 0.49 . As we can see, for $\theta>45^{\circ}$, the liquid that condenses on the rectangular grooves forms either drops $[D]$ or extended filaments with either positive $\left[\mathrm{F}^{+}\right]$or negative $\left[\mathrm{F}^{-}\right]$Laplace pressure. For $\theta<45^{\circ}$, the liquid forms the same three basic wetting morphologies, but in this case, they are in coexistence with a pinned wedge, i.e. $[\mathrm{D} / \mathrm{pW}],\left[F^{+} / \mathrm{pW}\right]$ and $\left[F^{-} / \mathrm{pW}\right]$.

From the above discussion it is clear that the morphology of a liquid in a rectangular groove is mainly affected by the contact angle of the liquid on the substrate and by the aspect ratio of the groove. The experimental observations have been combined with numerical and analytical calculations to construct the morphology diagram shown in Figure 6.5. In this figure, the full, dashed, and dotted lines represent the boundary and

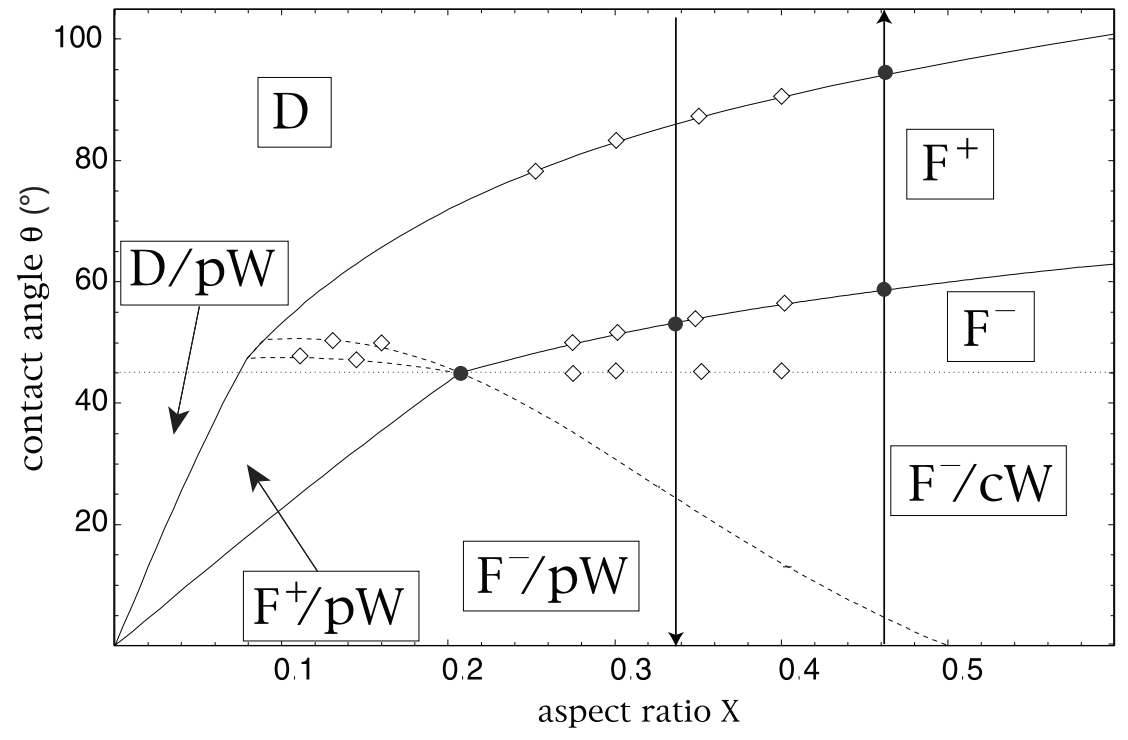

Figure 6.5: Morphology diagram of a rectangular groove as a function of groove aspect ratio $X$ and liquid contact angle $\theta<90^{\circ}$. (Image courtesy of R. Seemann.)

instability lines as obtained by the analytical theory. The small diamonds along those lines correspond to explicit numerical calculations of the three-dimensional boundary shapes. The dotted horizontal line divides the morphology diagram into two parts: the upper part corresponds to liquid morphologies with contact angle $\theta>45^{\circ}$, while the lower part corresponds to liquid morphologies with contact angle $\theta<45^{\circ}$. The lower solid line corresponds to filaments with zero Laplace pressure. All morphologies above 
and below this boundary line have positive and negative Laplace pressure respectively. The upper solid line is the stability line of filaments with positive Laplace pressure $\left[F^{+}\right]$.

\subsection{Wetting morphologies on rubber elastic substrates with rectangular grooves}

According to the prediction of the morphology diagram for rectangular grooves (Figure 6.5), switching between different morphologies can be achieved either by changing the contact angle of the liquid or by varying the aspect ratio of the groove. Given a specific non-deformable substrate, the aspect ratio is fixed and the only way to switch between different morphologies is by modifying the contact angle, e.g. using electrowetting [38, 87, 88]. On the other hand, on a rubber elastic substrate, switching between different liquid morphologies can be achieved by simply changing the substrate geometry, e.g. by bending or stretching it. In the following sections, we will not actively control the geometry of the rectangular grooves of the rubber elastic substrates, but we will restrict our study to the wetting phenomena that occur by the natural deformation of the grooves/ridges due to their interaction with the liquid.

Before proceeding to the fabrication of the PDMS substrates as described in Section 2.1.2 on page 46, the exact structure of each Si master was characterised by confocal microscopy. The necessary parameters for the characterisation are the aspect ratio and the groove width. It is important to mention here that, as opposed to what has been acknowledged so far regarding the aspect ratio on topographically structured rigid substrates, the aspect ratio considered in the rubber elastic substrates is not referring to the groove any more, but to the ridge, i.e. the aspect ratio is given by the height of the ridge $H$ with respect to its width $R$, as shown in Figure 6.6. Additionally, the ridge aspect ratios as well as the height of the ridges of the rubber elastic substrates have been chosen to be much larger than those on the rigid substrates.

Figure 6.7 shows a typical 3D image of a topographically structured Si master with rectangular grooves, acquired by confocal scanning laser microscopy.

One can observe that the topography in this image seems to be trapezoidal or triangular rather than rectangular. This is a result of the limitations of the confocal microscopy for the specific system: during scanning, we tried to use the optimum aperture setting 


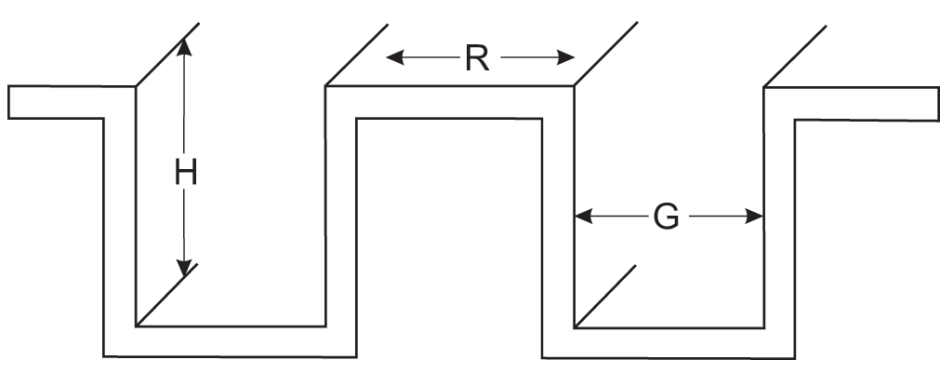

Figure 6.6: Sketch of a cross section of a topographically structured substrate with rectangular grooves. The parameters used for the characterisation of the sample are the groove width $G$, the ridge width $R$ and its height $H$.

in order to achieve a compromise between accurately rendering the substrate topography in sufficient contrast and retaining the resolution necessary to image the substrate structure while avoiding diffraction artifacts. As a result, an increased depth of field was sacrificed, therefore, the bottom of the grooves results distorted. Nevertheless, the information we obtain is sufficient in order to analyse the images and extract the necessary data.

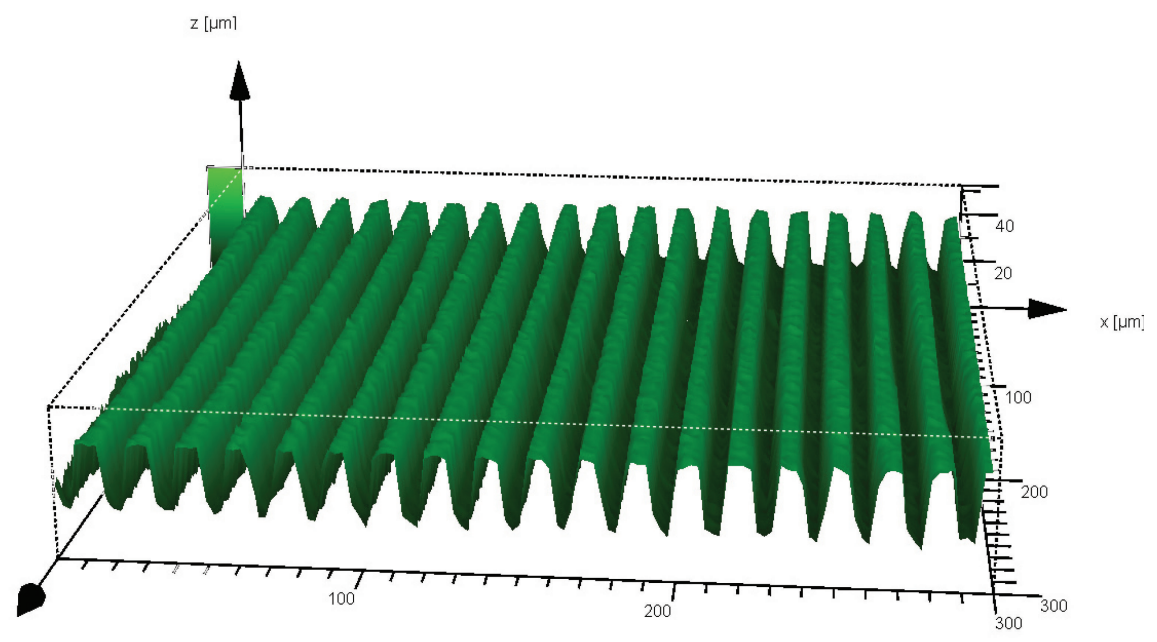

Figure 6.7: 3D projection of a confocal scanning laser microscopy image of a topographically structured Si master with rectangular grooves. 
The liquid used in order to study the wetting phenomena on the topographically structured rubber substrates is water. To create liquid morphologies, the samples were cooled below the dew point using a Peltier element, such that water from the atmosphere would condense from the vapour phase onto the structured PDMS. The experiments were monitored using a Leica microscope and time sequences of images were captured in order to follow the temporal evolution of the liquid morphologies.

\subsubsection{Results and discussion}

The condensation of water begins when the surface of the sample reaches a temperature below the dew point. First, water adsorbs on irregularities on the substrate surface. These irregularities can be dust particles, mechanical defects of the substrate or other residual stresses induced by chemical and thermal shrinkage when the elastomeric stamp was molded on the Si master and they increase the contact angle of the liquid on the substrate, acting as nucleation points for the growth of the droplets.

As one can observe in Figure 6.8, the system of droplets seems to be rather monodisperse. The explanation for this is that water condenses on the substrate with the same

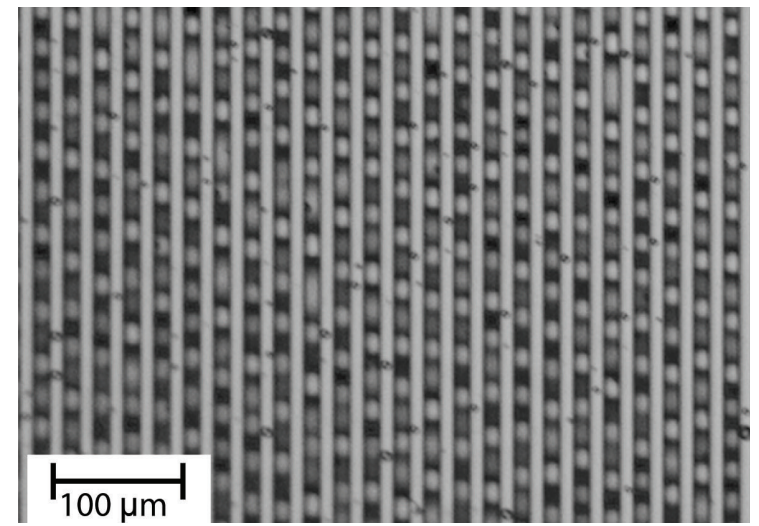

Figure 6.8: Optical micrograph of monodisperse water droplets in rectangular grooves of a topographically structured PDMS substrate.

rate, thus droplets are nucleated at the same time and grow with the same rate. Moreover, droplets seem to preferentially adsorb in the grooves rather than on the ridges. A possible explanation for that is that the bottom of the grooves has a slightly lower temperature than the top due to the PDMS being an excellent thermal insulator. 
When a droplet in a groove increases its volume such that it touches both walls of the groove where it is contained, the liquid, due to its large surface tension, applies an attractive force on the walls, respectively the ridges of the groove. Due to this force, the ridges bend towards each other and the morphology of the overall liquid pattern changes. This effect is stronger the closer the droplets are to each other: the more droplets there are per unit groove length, the larger the sum of the force applied on the ridges, enhancing thus their deformation. An example of this affirmation can be seen in Figure 6.9

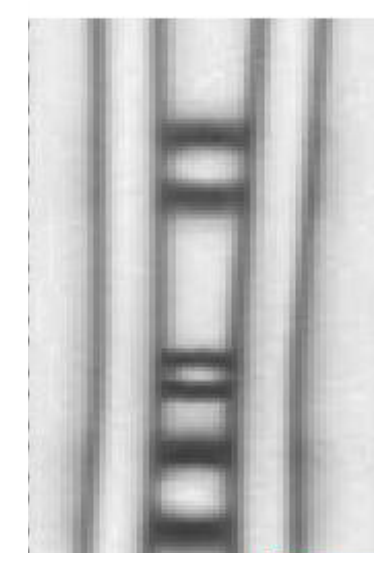

Figure 6.9: Close-up of an optical micrograph of nearby droplets deforming the ridges of the groove in which they are contained.

After water droplets have appeared on the substrate, the intermediate stage of condensation begins, where the droplets continue to grow in size until they reach their neighbouring droplets with which they coalesce, forming filaments with positive mean curvature. Such a filament, due to its positive Laplace pressure, exerts a repulsive force to the confining ridges that define the groove. The local widening of one groove due to the outwards bending of its ridges, leads to the local narrowing of its neighbouring groove. The filament that is in the narrowed groove "feels" the force that its confining ridges apply on it and, under this force, it is constrained to move along the groove towards a region where it itself will exert a repulsive force to its confining ridges, constraining the laterally neighbouring filament to relocate inside its groove. This local phenomenon spreads like a domino effect on the whole area of the structured substrate. Eventually, as the filaments relocate inside their groove, the force they apply on their neighbouring ridges is so large that the ridges come very close and, due to the adhesion 
forces between them, they "snap" to each other. This mechanism appears at the late stage of condensation (Figure 6.10) and it gradually impedes the contact between the filaments and thus decreases the number of coalescing filaments until coalescing eventually stops completely. At this point, any further condensation of water will not lead to a rearrangement of the filaments, but the volume of the single filaments will increase, leading to their overspilling on the ridges of the grooves, as can be seen in the area inside the red circle in Figure 6.10.

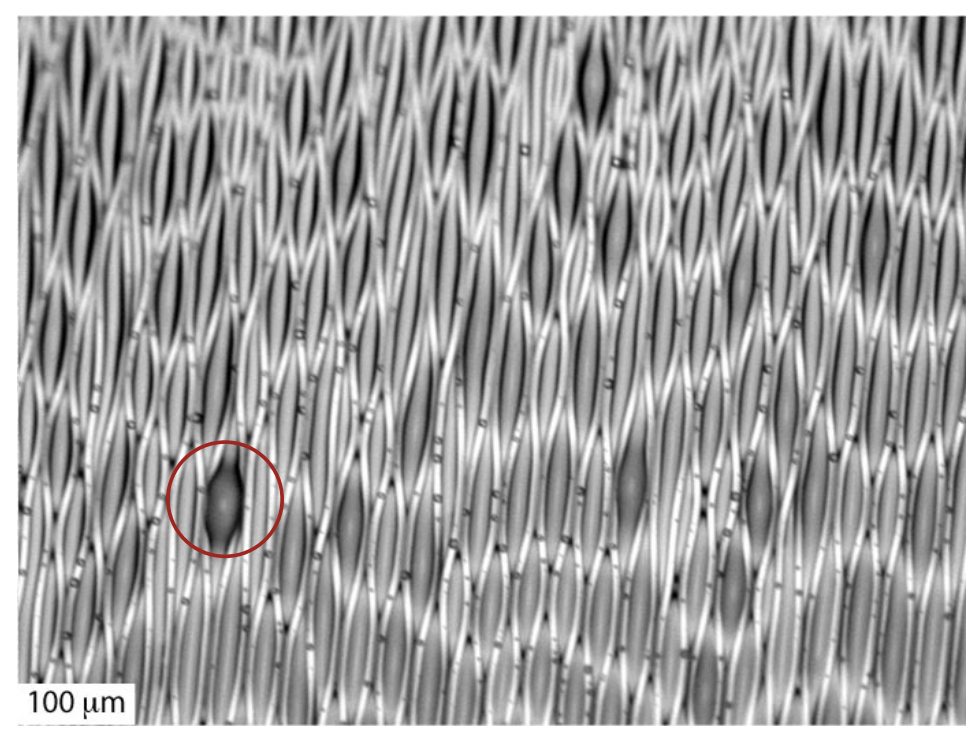

Figure 6.10: Optical micrograph at the late stage of water condensation on a topographically structured PDMS substrate with high ridge aspect ratio. Filaments can be observed to be arranged in a laterally periodic pattern. Highlighted in the red circle is a filament overspilling on the ridges.

The final pattern is expected to depend on various parameters such as the substrate elasticity, geometry and wettability of the given substrate by the specific liquid. In the following we have focused our study on actively tuning the geometry and the wettability.

The influence of the geometry can be examined by actively tuning two independent parameters: the ridge aspect ratio and the groove width.

Figure 6.11 depicts liquid morphologies formed by the condensation of water on a rubber elastic substrate with rectangular grooves of large ridge aspect ratio and low groove width. We can observe that most filaments seem to have similar lengths and the lateral distribution of the filaments presents a periodic pattern. The histogram of the 
normalized filament length is a narrow Gaussian with low standard deviation (Figure 6.13. (a), blue histogram), located towards the low values for the filament length. That means that there is a preferred size in which the filaments grow, after which, due to the interaction between the ridges of the groove, the coalescence between the filaments stops.

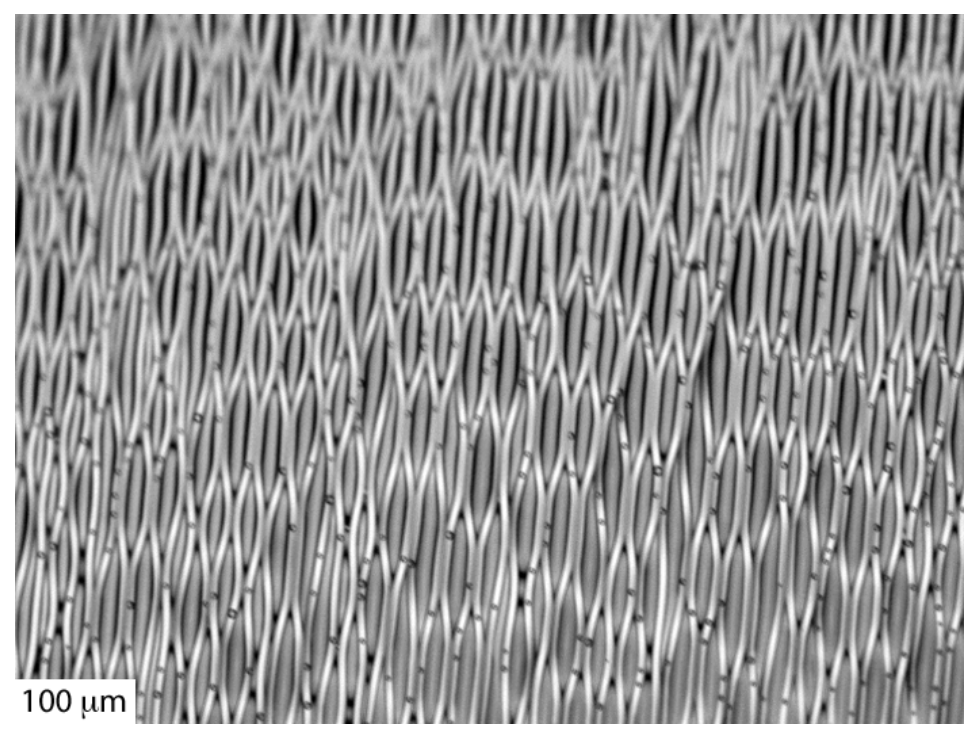

Figure 6.11: Optical micrograph of the liquid morphologies on a PDMS substrate with rectangular grooves of large ridge aspect ratio and low groove width.

Figure 6.12 shows an optical micrograph of water filaments condensed in PDMS rectangular grooves of low ridge aspect ratio and large groove width. In this case, we observe that filaments appear in various sizes and that the size of an average filament appears to be larger that the one found in the case depicted in Figure 6.11. Furthermore, the ordering effects do not seem to be dominant in this system. This is due to the fact that, at a certain threshold, the Laplace pressure is not sufficient any more to bend the ridges enough, so that the interaction between the laterally neighbouring filaments is less strong than the interaction between the filaments in the same groove. In this case, we derive a wider histogram that is located towards the higher filament lengths (Figure 6.13. (a), red histogram) in comparison to the histogram for the rectangular grooves of large ridge aspect ratio and low groove width.

Similar results to the ones discussed above for a wide range of aspect ratios and groove widths are summarized in Figure 6.13.(b), where the contour plot of the filament length as a function of ridge aspect ratio and groove width is shown. We can observe 


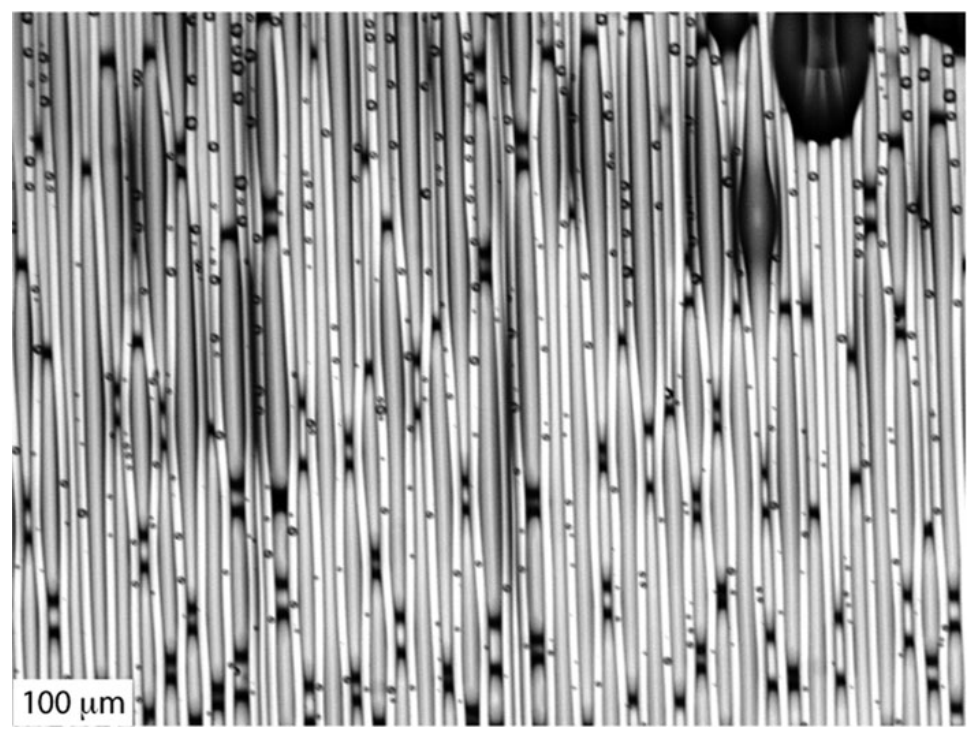

Figure 6.12: Optical micrograph showing liquid morphologies on a PDMS substrate with rectangular grooves of low ridge aspect ratio and large groove width.

that the highest value of filament length can be achieved by increasing the groove width and decreasing the ridge aspect ratio, while, as we move towards smaller groove widths and higher aspect ratios, the preferred filament length becomes smaller.

From the above we can summarize and deduct that the final pattern of the liquid morphologies on rectangular grooves of rubber elastic substrates depends on the geometry of the groove and specifically on:

- the ridge aspect ratio that is, to a certain extent, equivalent to the elastic properties of the substrate. For higher aspect ratios, the ridges become more flexible and thus the interaction between the filaments is stronger,

- the groove width, i.e. the distance between the ridges. A smaller groove width forces the liquid filament into a larger curvature, respectively higher Laplace pressure applied on the ridges, thus, the interaction between the filaments is stronger the smaller the groove width. 

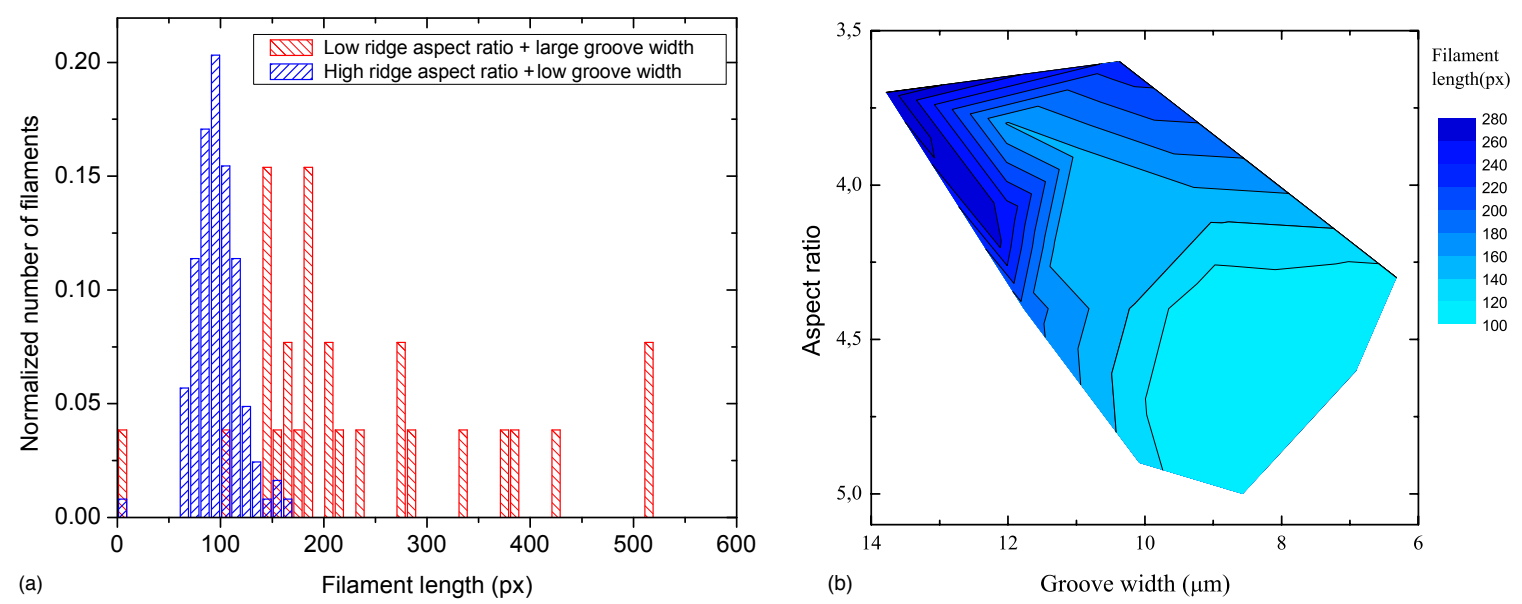

Figure 6.13: (a) Histogram of the normalized filament length for the two cases shown in Figure 6.11 and Figure 6.12. The distribution for the sample with the lower aspect ratio and higher groove width (Figure 6.12) is wider and presents a higher mean value than that for the sample with the higher aspect ratio and the smaller groove width (Figure 6.11). (b) Contour plot of the filament length as a function of ridge aspect ratio and groove width. The highest value of filament length can be achieved by increasing the groove width and decreasing the ridge aspect ratio, while, towards lower groove widths and higher aspect ratios, the preferred filament length becomes smaller.

Finally, we want to explore the influence of the contact angle on the ordering behaviour of the condensed water morphologies on the rubber elastic grooves. Based on the morphology diagram for rectangular grooves on rigid substrates (Figure 6.5), what we expect in case of a low contact angle is filaments with a negative mean curvature $\left[F^{-}\right]$. Such filaments, due to their negative Laplace pressure, are predicted to exert an attractive force on the ridges and a periodic pattern is expected to arise due to this force.

To obtain contact angles lower than $60^{\circ}$, we modified our structures chemically. This was achieved by exposing our PDMS substrates to oxygen plasma for a certain amount of time, which causes the formation of $\mathrm{SiOx}$ groups during the plasma mediated oxidation and the formation of a hydrophilic silica-like surface on the PDMS. However, the hydrophilic surface is unstable and low molecular weight chains diffuse from the bulk of the PDMS and cover up the thermodynamically unstable surface. This leads to a gradual recovery of the initial contact angle and makes the investigation of the wetting phenomena on hydrophilic surfaces much more complicated than on hydrophobic ones.

Furthermore, a periodic structure was impeded from arising: soon after water con- 


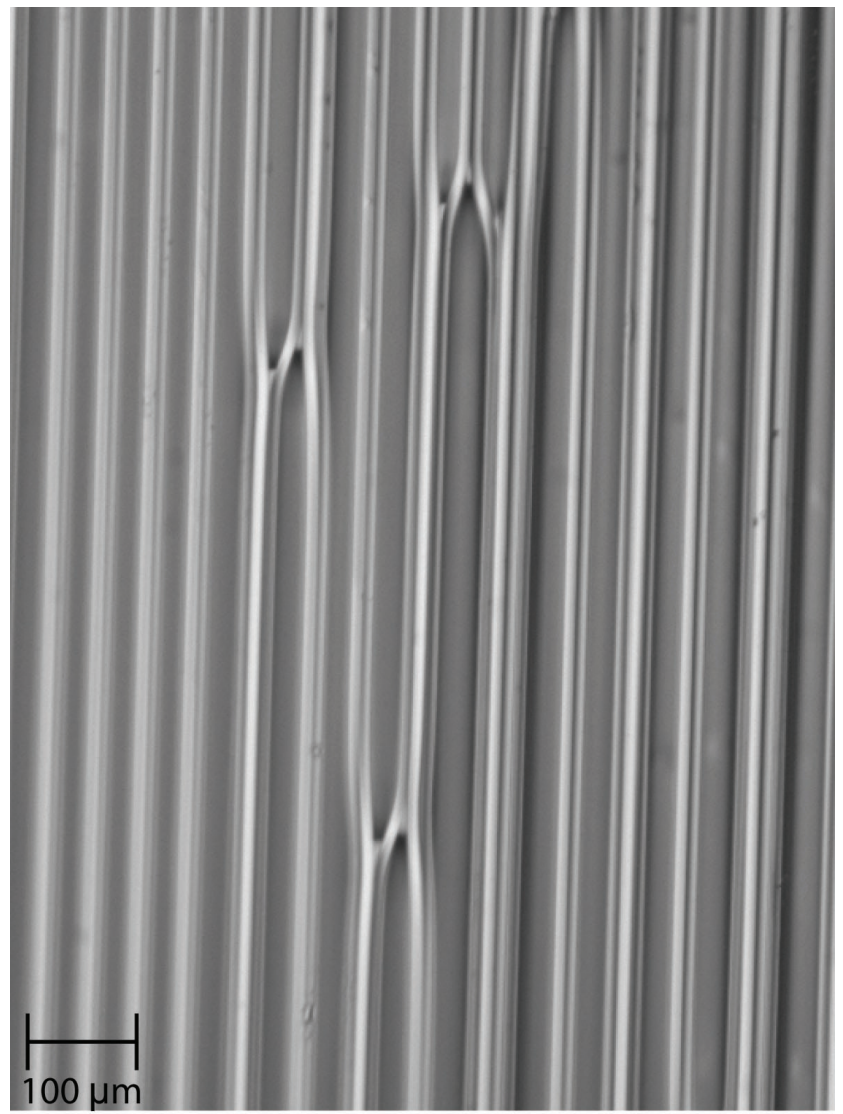

Figure 6.14: Optical micrograph of a hydrophilized PDMS substrate after water condensation has taken place. The ridges collapse along the length of the groove similar to a closing zipper.

densation was started, a collapse of the ridges along the length of the groove was observed as one can see in Figure 6.14, and no wetting effects would take place after that. A possible explanation for this effect is sketched in Figure 6.15 and might be the following: when waters starts to adsorb on the grooves, filaments with negative Laplace pressure are formed, which apply an attractive force to the ridges of the groove. At the same time, the liquid, due to its low contact angle, wets the walls of the groove. Due to the strong capillary forces between the liquid adsorbed at the upper part of the ridges, which are also the most flexible, these two "snap" together. By doing so, they decrease the cross-section area of the groove and consequently of the liquid. Since the liquid volume has to remain constant, the liquid spreads along the groove. As it spreads, it causes the ridges to collapse due to the capillary forces along the whole groove similar to a closing 
zipper. This "zipper effect" destroys any periodic structure that could be detected on such a substrate.

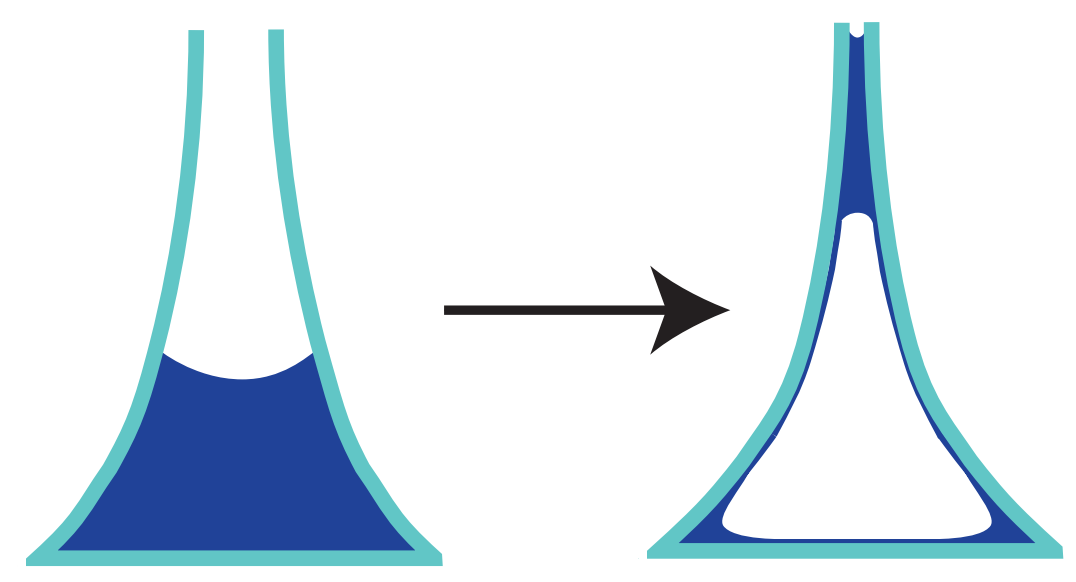

Figure 6.15: Schematic rapresentation of the "zipper effect". 


\section{Chapter 7}

\section{Summary and outlook}

So far, the dewetting phenomena had been studied thoroughly on rigid substrates, thus, the mechanisms that dominate the dewetting process are well known. Our aim has been to extend such knowledge to non-rigid substrates and examine how their deformability affects the dewetting process.

We reported our results of a systematic study of the dewetting of thin polystyrene films on elastic and on viscous substrates as a function of the substrate viscoelastic properties.

We showed that the dewetting velocity exhibits a different behaviour based on whether the substrate's elastic or viscous properties dominate the system. The curve of the dewetting rates on the elastic substrates indicates that energy dissipation takes place also at the liquid/rubber interface, whereas this could not be observed for the viscous substrates, where the developed rim grows linearly with time. Furthermore, the dewetting velocity on the elastic substrates increases with increasing substrate elasticity, whereas, for the viscous substrates, it exhibits a significantly more complicated behaviour as a function of substrate viscosity. The latter, though essentially explained by existing theories, presents some peculiarities that have not been completely understood yet.

Additionally, we characterised the overall shape of the rim surrounding the growing hole, i.e. both the air/PS and PS/substrate interface. We found that its shape is strongly correlated to the viscoelastic properties of the substrate, influencing therefore the dewetting process. The information obtained from the analysis of the rim profiles matched accurately the one from the dewetting rates discussed above. 
Parallel to the experimental progress concerning the dynamics of polymer thin films on rubber elastic substrates, the corresponding theory is in a developing state as well. In a first attempt to explain our results with the given theory of linear elasticity, we disregarded any tangential forces that might be acting on the liquid/rubber interface. For small hole radii, we observed a satisfactory agreement between the experimentally measured and theoretically calculated deformation. Nevertheless, an increasing quantitative deviation was observed for increasing hole radii. Thus, a first attempt to explain the deformation during hole growth by implementing only the Laplace pressure inside the liquid has given results that do not fully match our experimental observations. Therefore, it is evident that further dynamics aspects need to be additionally considered. The linear elasticity theory might not be sufficient to account for these effects, therefore it might be appropriate to model our system based on the non-linear elasticity theory.

In collaboration with the group of A. Münch and B. Wagner, a numerical model has been developed in order to explain theoretically the dewetting on a viscous substrate. The focus in this case has been placed on the direct comparison of the shape of the interfaces of a moving rim to the experimental results and its dependence on the ratios of the viscosities and the surface tension. A satisfactory agreement between experiment and theory has been achieved, although with some minor deviations between the theoretical predictions and the experimental results. That is partly because, to experimentally measure with great accuracy certain parameters, is a demanding process. Therefore, the experimental uncertainty of many parameters might lead to a deviation between the actual experimental value and the one implemented in the model. Furthermore, the role of slippage in the system has not yet been analysed. Slippage has an impact on many aspects of liquid flow, such as energy dissipation at the liquid/solid interface, the propagation of the contact line and the liquid profiles. In the present work we have assumed no slip between the substrate and the liquid. It is of interest to verify this assumption and, in case of it turning out false, it is important to implement in our theory a component that systematically describes how the film dynamics and profiles change in time as the slip length is varied.

Furthermore, we studied the stresses that sessile nanodroplets apply on the elastic substrates, generating a characteristic craterlike shape on the substrate. We analysed the complete shape of the droplet, i.e. the shape of the air/PS interface, the deformation at the dry side of the three phase contact line (TPCL) and the deformation below the droplet. We compared the shape of our droplets to existing theories and we found them 
to be in good agreement. Moreover, we have developed a technique that, in combination with existing theoretical models, allows us to derive the substrate elastic properties.

The knowledge gained by the study of thin polymer films on planar viscoelastic substrates has been applied on the examination of wetting phenomena on structured viscoelastic substrates. Specifically, part of this work has been concerned with the investigation of water morphologies in rectangular grooves of rubber elastic substrates. We showed that the pattern obtained by the condensation of water on such substrates depends on the geometry of the groove i.e. in our case, on the ridge aspect ratio and on the groove width. This study is still at an early stage and there are challenges to overcome. Regarding the wetting liquid, the use of polymers, e.g. Polystyrene with an adequate chain length such that it is glassy at room temperature, would be very useful, as one would not have to be concerned with water's relatively high vapour pressure anymore. Thus, since the liquid structures could become glassy on demand by quenching them at room temperature, new techniques could be employed other than optical microscopy in order to study the structures in more detail, e.g. AFM. Finally, even though important basic knowledge on wetting phenomena was gained by tuning the substrate wettability and elasticity, these are definitely key parameters whose role has to be investigated more profoundly. 

Appendices 



\section{Appendix A}

\section{Notes on the preparation of the PDMS substrates}

In order to be able to successfully compare our results for the different substrates and extract safe assumptions regarding their elastic properties, it is important that the preparation and storage methods do not affect the reproducibility of our results.

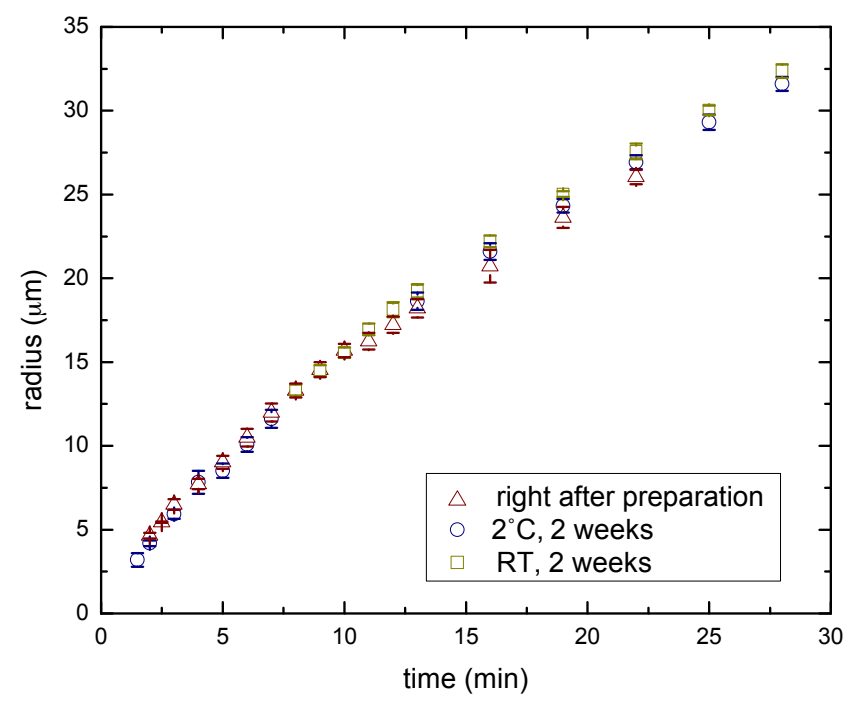

Figure A.1: Dewetting rates of a $150 \mathrm{~nm}$ thick PS(17.4k) film on SG184 substrates of different aging. 
One concern was the aging of the substrates. Even though fresh samples were produced regularly, a storage time of some days was inevitable. To control the aging of the samples, we compare the dewetting rates of PS on three SG184 substrates with different storage time and method: one substrate was used right after curing, the other was stored at room temperature for two weeks and the third one was stored at $2{ }^{\circ} \mathrm{C}$ for two weeks. The results are shown in Figure A.1. The dewetting rates of PS on the three substrates do not exhibit any significant variation, therefore no decisive modification of the substrate elasticity took place due to aging within the time-lapse of two weeks.

Furthermore, to control if the presence of possible non cross-linked PDMS molecules inside the cured rubber affected the resulting elasticity of the substrate, we followed a well-established method of leaching the PDMS after curing in order to remove the residual non cross-linked polymers from the otherwise cross-linked matrix. The sol-

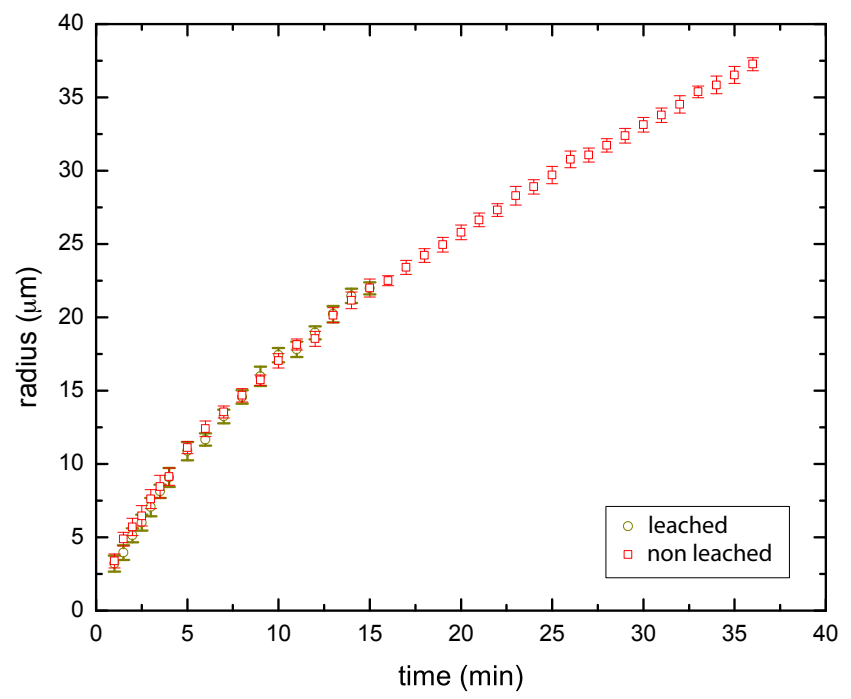

Figure A.2: Dewetting rates for a $150 \mathrm{~nm}$ thick PS(17.4k) film dewetting on SG184 at $120^{\circ} \mathrm{C}$.

vents used for leaching the PDMS were toluene, a good solvent for PDMS, adequate to remove the non cross-linked molecules and ethanol, a solvent which is miscible with toluene, adequate to remove any toluene molecules remaining in the cross-linked matrix of PDMS. The sample was immersed in toluene for 24 hours, subsequently in ethanol for 10 hours and finally again in toluene for 24 hours. Initially, bulk samples of PDMS were 
leached with this method and their weight was measured before and after leaching. An insignificant decrease in weight in the order of $1 \%$ was measured. Subsequently, films of PDMS on $\mathrm{Si}$ wafers as prepared for our experiments were leached following the same protocol. We then compared the dewetting rates of PS on a leached and a non-leached sample. The results are shown in Figure A.2. We observe no significant difference in the dewetting rates. Nevertheless, on the non-leached sample, the growing holes maintained their circular shape for longer time. This is probably to the fact that successive swelling and deswelling of PDMS in the solvents deteriorated the quality of the surface, which lead to more pinning sites for the three-phase contact line of the dewetting liquid.

The curing time can also play a role in the final elasticity of the substrate. The manufacturers of SYLGARD suggest a curing time of $10-15$ minutes at $150^{\circ} \mathrm{C}$. Nevertheless, to ensure a cross-linking as complete as possible, we use longer times. As one can observe in Figure A.3, judging from the nearly similar dewetting rates, curing the PDMS for at least 30 minutes at $170^{\circ} \mathrm{C}$, results in practically the same elasticity as for a 90 minute curing at $170^{\circ} \mathrm{C}$. Therefore, at the specific temperature, a curing time of $30-90$ minutes assures a practically similar cross-linking ratio.

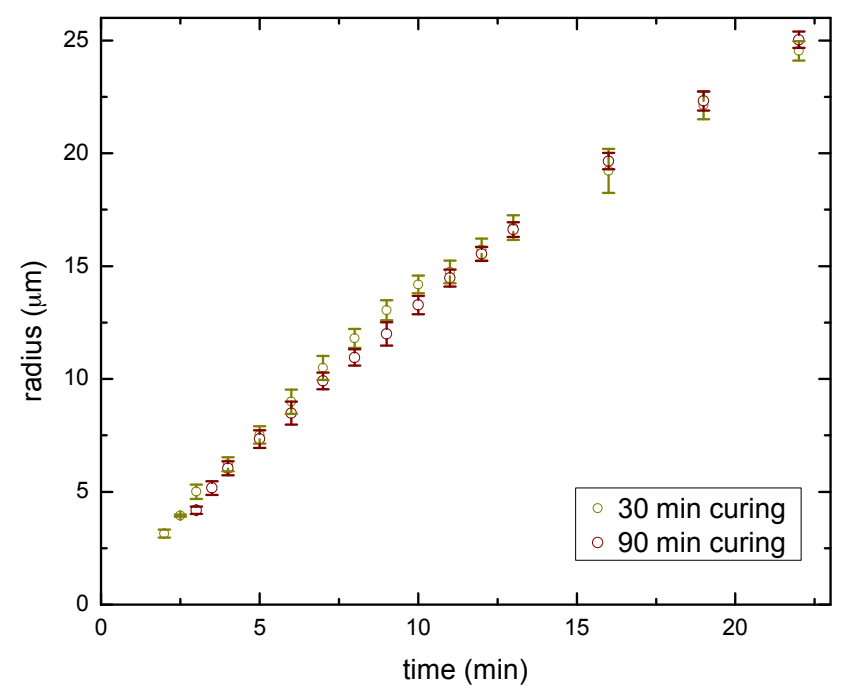

Figure A.3: Dewetting rates for a $150 \mathrm{~nm}$ thick PS(17.4k) film dewetting on SG184 at $120^{\circ} \mathrm{C}$.

SG186 is significantly more viscous than SG184. When spin-coated on a Si wafer, it 
results into a thicker PDMS film than that of SG184. Since PDMS is a good thermal insulator, to avoid obtaining different results due to the difference in surface temperature rather than in elasticity, we dilute SG186 in toluene as described on page 41. To ensure that the dilution in toluene does not alter the viscoelastic properties of the resulting PDMS film, we compare the dewetting rates of PS on two substrates of SG186: one diluted in toluene and the other not. In this case, the annealing temperature of PS was reached very slowly, i.e. with a rate of $1^{\circ} \mathrm{C} / \mathrm{min}$, so that enough time was given to the surface of the thicker SG186 to reach the desired temperature. As can be seen in Figure A.4, no significant difference was observed in the dewetting rates of PS on the two SG186 substrates.

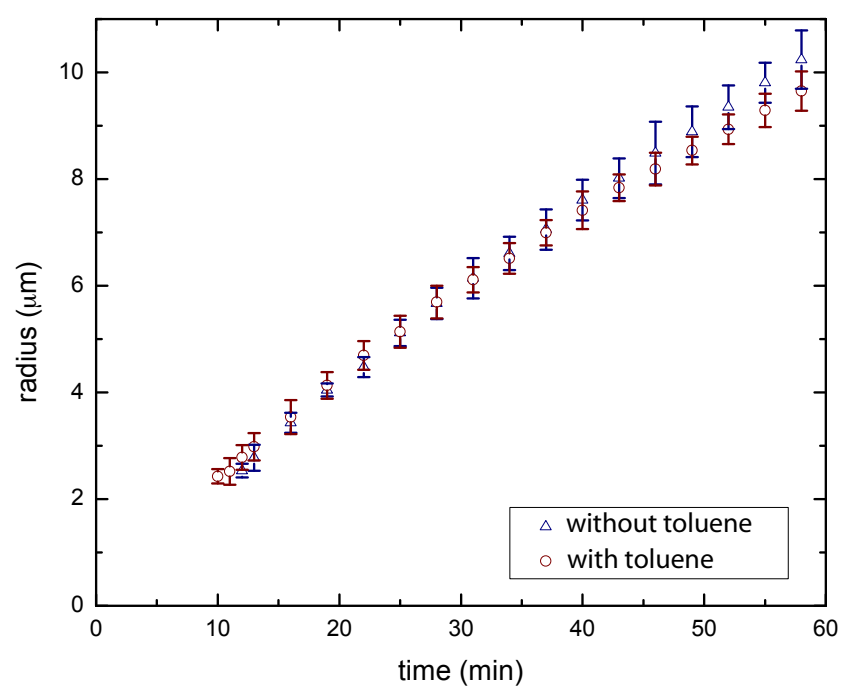

Figure A.4: Dewetting rates for a $150 \mathrm{~nm}$ thick PS(17.4k) film dewetting on SG186 at $120^{\circ} \mathrm{C}$. 


\section{Appendix B}

\section{Model - Rubber elastic substrates}

We restrict our theoretical calculations to sufficiently late times after nucleation such that the radius of the hole has become reasonably large compared to the width of the rim to assume that the shape of the dewetting front is translationally invariant with respect to the direction perpendicular to the dewetting front (the $z$-direction with respect to our convention, cf. Figure 4.5. (c) on page 79) and consider a two dimensional crosssection of the dewetting front in the $x z$-plane. As the slope of the liquid/air interface in the rim and in the transition region between the rim and the unperturbed flat film is small compared to unity, we suppose that the pressure gradient inside the liquid is mainly horizontal, i.e. in the $x$-direction. Hence, the pressure difference between two points with the same $x$ - coordinate, one close to the liquid/air interface and another close to the substrate is neglected.

Furthermore, we assume that the deformation of the PDMS substrate is caused only by the pressure in the film. In particular this implies that tangential stresses acting on the substrate wall are disregarded. For a known interfacial tension $\gamma$, the Laplace pressure $p$ inside the liquid can be calculated from the profile $z(x)$ of the liquid film as

$$
p(x)=\gamma c(x),
$$

where $c$ is the local curvature

$$
c(x)=-\frac{\ddot{z}(x)}{\left(1+\dot{z}^{2}(x)\right)^{3 / 2}}
$$


of the liquid-air interface at the position $x$. In linear elastic theory the deformation $\mathbf{u}(\mathbf{r})$ at a point $\mathbf{r}=(x, y, z)$ of the semi-infinite elastic substrate $z \leq 0$ can be calculated from a superposition of deformations caused by point-like normal loads distributed on the surface of the elastic substrate. The solution of Boussinesq and Cerruti [89] for a point-like normal load of unity placed at the origin of the Cartesian coordinate system reads

$$
\begin{aligned}
&\left(\begin{array}{l}
u_{x} \\
u_{y} \\
u_{z}
\end{array}\right)= \\
& \frac{1}{4 \pi E}\left(\begin{array}{lll}
\frac{b}{r}-\frac{x^{2}}{r^{3}}-\frac{a x^{2}}{r(r+z)^{2}}-\frac{a z}{r(r+z)} & \frac{x y}{r^{3}}-\frac{a x y}{r(r+z)^{2}} & \frac{x z}{r^{3}}-\frac{a x}{r(r+z)} \\
\frac{x y}{r^{3}}-\frac{a x y}{r(r+z)^{2}} & \frac{b}{r}-\frac{y^{2}}{r^{3}}-\frac{a y^{2}}{r(r+z)^{2}}-\frac{a z}{r(r+z)} & \frac{y z}{r^{3}}-\frac{a y}{r(r+z)} \\
\frac{x z}{r^{3}}+\frac{a x}{r(r+z)} & \frac{y z}{r^{3}}+\frac{a y}{r(r+z)} & \frac{b}{r}-\frac{z^{2}}{r^{3}}
\end{array}\right)
\end{aligned}
$$

where $a=1-2 \nu$ and $b=2(1-\nu)$, while $E$ and $\nu$ denote the Young's modulus and the Poisson ratio respectively. Since PDMS rubber can be regarded as nearly incompressible, we have $\nu=1 / 2$. Hence, $a=0$ and $b=1 / 2$ and the Greens function (Equation B.3) simplifies to

$$
\begin{aligned}
&\left(\begin{array}{l}
u_{x} \\
u_{y} \\
u_{z}
\end{array}\right)= \\
& \frac{1}{4 \pi E}\left(\begin{array}{lll}
\frac{1}{r}-\frac{x^{2}}{r^{3}} & \frac{x y}{r^{3}} & \frac{x z}{r^{3}} \\
\frac{x y}{r^{3}} & \frac{1}{r}-\frac{y^{2}}{r^{3}} & \frac{y z}{r^{3}} \\
\frac{x z}{r^{3}} & \frac{y z}{r^{3}} & \frac{1}{r}-\frac{z^{2}}{r^{3}}
\end{array}\right)
\end{aligned}
$$

Because of the tanslational invariance with respect to the $y$-direction we may integrate the left side of Equation B.4 over $y$ and arrive at the deformation

$$
u_{z}(x)=\frac{1}{2 \pi} \ln |x|
$$

of the interface at $x$ due to a unity point load on the surface $z=0$ at $x=0$. The contour 
of the deformed elastic substrate is then given by the convolution

$$
\delta z\left(x_{o}\right)=\frac{1}{2 \pi} \int_{x_{c}}^{x_{f}} \mathrm{~d} x p(x) \ln \left|x-x_{o}\right|-\frac{P}{2 \pi} \ln \left|x_{c}-x_{o}\right|
$$

of the normal load $p(x)$ and the 2D Greens function (Equation B.5). The point $x=x_{c}$ on the surface of the elastic substrate is the position of the contact line while $x_{f}$ represents a point which is sufficiently far away from the rim where the film is flat and hence $p=0$. The second term corresponds to the point load $P=\int_{x_{c}}^{x_{f}} \mathrm{~d} x p(x)$ of the contact line at $x=x_{c}$. The total pressure load then integrates to zero.

The results of the comparison between the experimentally and the theoretically derived rim profiles are given on page 84 in Chapter 4. 



\section{Appendix $\mathrm{C}$}

\section{Model - Liquid substrates}

To numerically model the case of PS dewetting on PMMA, we state a free boundary problem that can describe the dewetting dynamics as well as the stationary states for the two-layer PS-PMMA system. At this point we do not account for elastic properties in any of the two liquids, thus we assume that we are in a parameter regime where our experiments allow the liquids to be considered Newtonian. That means that the model we present can explain our system only in the case of PMMA and PS molecular weights below or around the entanglement length. Furthermore, we consider the typical height scale $H$ to be much smaller than the typical length scale $L$ so that $\epsilon=\frac{H}{L}$ is a small parameter. The dynamics of the two liquids is dominated by viscous dissipation, surface tension, and the long-range van der Waals forces.

We solve the Navier-Stokes equation for the PS and the PMMA

$$
\rho_{i}\left(\partial_{t} \mathbf{u}_{i}+\mathbf{u}_{i} \cdot \nabla \mathbf{u}_{i}\right)=\nabla \cdot \sigma_{i}+\mathbf{f}_{i} \quad \text { in } \Omega_{i} \quad(i=A, B)
$$

where $\mathbf{u}_{i}, i=A$ and $i=B$, is the velocity of liquid in the domain $\Omega_{A}$ (PS) and $\Omega_{B}$ (PMMA), respectively, as shown in Figure 5.2 on page 90 . The stress tensor for the two Newtonian fluids is

$$
\sigma_{i}=-p_{i} \mathbb{I}+\mu_{i}\left(\nabla \mathbf{u}_{i}+\nabla \mathbf{u}_{i}^{\top}\right)
$$

Using the functions $h_{A}$ and $h_{B}$ as defined in Figure 5.2, we define the domains $\Omega_{A}(t)=$ $\left\{(r, y) \in \mathbb{R}^{2}: h_{B}(t, r)<y<h_{A}(t, r)\right\}$ and $\Omega_{B}(t)=\left\{(r, y) \in \mathbb{R}^{2}: 0<y<h_{B}(t, r)\right\}$. The position of the silicon/PMMA substrate is located at $\Gamma_{0}=\{y=0\}$, whereas the 
position of the PMMA/PS and PS/air interfaces are located at $\Gamma_{B}=\left\{y=h_{B}\right\}$ and $\Gamma_{A}=\left\{y=h_{A}\right\}$ respectively.

The two domains are coupled by a stress jump condition

$$
\left(\sigma_{A}-\sigma_{B}\right) \cdot \mathbf{n}=2 \gamma_{B} c_{B} \mathbf{n}_{B}
$$

where $\mathbf{n}_{B}$ is a normal vector onto the boundary $\Gamma_{B}, c_{B}$ is the mean curvature and $\gamma_{B}$ the coefficient for the surface tension between PS and PMMA. Furthermore, we have a kinematic condition

$$
\left(\partial_{t} h_{B} \mathbf{j}-\mathbf{u}_{B}\right) \cdot \mathbf{n}_{B}=0
$$

which fixes the normal component of the velocity of the interface; $\mathbf{j}$ being the unit vector $\mathbf{j}=(1,0,0)$. The velocity $\mathbf{u}_{B}$ is continuous across the interface $\Gamma_{B}$. At the interface $\Gamma_{A}$, we have the corresponding stress condition $\sigma_{A} \cdot \mathbf{n}_{A}=-2 \gamma_{A} c_{A} \mathbf{n}_{A}$ and kinematic condition $\left(\partial_{t} h_{A} \mathbf{j}-\mathbf{u}_{A}\right) \cdot \mathbf{n}_{A}=0$.

By applying the lubrication approximation, this set of coupled partial differential equations can be simplified to a set of partial differential equations for the functions $h_{A}$ and $h_{B}$ alone. Various aspects of such models have been studied earlier, e.g. by Thiele et al. [90]. In this model we focus in the direct comparison of the shapes of the interfaces $h_{A}$ and $h_{B}$ of a moving rim with the experimental results and study their dependence on the parameters $\alpha$ and $\gamma$, which denote respectively the relative viscosity and relative surface tension of PMMA with respect to the PS.

Finally, the derivative of the intermolecular van der Waals potential from which we derive the force $\mathbf{f}_{i}$ is defined by

$$
V_{A}=-V_{B}=\frac{8 \phi^{*}}{3} \frac{h^{* 2}}{\left(h_{A}-h_{B}\right)^{3}}-\frac{h^{* 8}}{\left(h_{A}-h_{B}\right)^{9}}
$$

After non-dimensionalization with suitable scales, we obtain the following problem: for the given initial data $0<h_{B}(0, r)<h_{A}(0, r)$ we are invited to find $h_{B}(t, r)$ for the PMMA/PS interface and $h_{A}(t, r)$ for the PS/air interface, such that

$$
\partial_{t} h_{i}=\operatorname{div} \sum_{j=A, B} Q_{i j} \operatorname{grad} p_{j} \quad \text { with } \quad p_{i}=-2 \tilde{\gamma}_{i} c_{i}+V_{i}\left(h_{2}-h_{1}\right)
$$


where $\tilde{\gamma_{B}}=\gamma=\frac{\gamma_{B}}{\gamma_{A}}, \tilde{\gamma}_{A}=1$ and $i=A, B$. The mean curvature is

$$
2 c_{i}=\frac{h_{i}^{\prime \prime}}{\left(1+\epsilon^{2} h_{i}^{\prime 2}\right)^{3 / 2}}+\frac{h_{i}^{\prime}}{r\left(1+\epsilon^{2} h_{i}^{\prime 2}\right)^{1 / 2}}
$$

with $h_{i}^{\prime}=\partial_{r} h_{i}$ and $h_{i}^{\prime \prime}=\partial_{r}^{2} h_{i}$. We are only interested in axial symmetric solutions $h_{A}$ and $h_{B}$, so that we can use the corresponding versions of divergence and gradient. The symmetric mobility matrix is thus defined

$$
\left(\begin{array}{ll}
Q_{A A} & Q_{A B} \\
Q_{B A} & Q_{B B}
\end{array}\right)=\frac{1}{\alpha}\left(\begin{array}{cc}
\frac{\left(h_{A}-h_{B}\right)^{3}}{3}(\alpha-1)+\frac{h_{A}^{3}}{3} & \frac{h_{1}^{2}}{2}\left(h_{A}-\frac{h_{B}}{3}\right) \\
\frac{h_{B}^{2}}{2}\left(h_{A}-\frac{h_{B}}{3}\right) & \frac{h_{B}^{3}}{3}
\end{array}\right)
$$

As input parameters for the numerical calculations we used the input parameters derived from our experiments such as surface energies, viscosities, contact angle and film heights.

The comparison between the experimental results and the numerical simulations is discussed on page 99 in Chapter 5 . 



\section{Bibliography}

[1] D. Ansell, "surface tension.png." http://www.thenakedscientists.com/.

[2] K. Jacobs, R. Seemann, G. Schatz, and S. Herminghaus, "Growth of holes in liquid films with partial slippage," Langmuir, vol. 14, pp. 4961-4963, Sept. 1998.

[3] N. Giordano and J. Cheng, "Microfluid mechanics: progress and opportunities," Journal of Physics: Condensed Matter, vol. 13, no. 15, pp. R271-R295, 2001.

[4] P. Mitchell, "Microfluidics downsizing large-scale biology," Nat Biotech, vol. 19, no. 8, pp. 717-721, 2001.

[5] Cannon, T. Kuo, P. W. Bohn, and J. V. Sweedler, "Nanocapillary array interconnects for gated analyte injections and electrophoretic separations in multilayer microfluidic architectures," Analytical Chemistry, vol. 75, pp. 2224-2230, May 2003.

[6] P. Ramirez, S. Mafe, A. Alcaraz, and J. Cervera, "Modeling of pH-Switchable ion transport and selectivity in nanopore membranes with fixed charges," The Journal of Physical Chemistry B, vol. 107, pp. 13178-13187, Nov. 2003.

[7] P. Kohli, C. C. Harrell, Z. Cao, R. Gasparac, W. Tan, and C. R. Martin, "DNAFunctionalized nanotube membranes with Single-Base mismatch selectivity," Science, vol. 305, pp. 984-986, Aug. 2004.

[8] K. B. Jirage, J. C. Hulteen, and C. R. Martin, "Effect of thiol chemisorption on the transport properties of gold nanotubule membranes," Analytical Chemistry, vol. 71, pp. 4913-4918, Nov. 1999.

[9] T. Kuo, L. A. Sloan, J. V. Sweedler, and P. W. Bohn, "Manipulating molecular transport through nanoporous membranes by control of electrokinetic flow: Effect 
of surface charge density and debye length," Langmuir, vol. 17, pp. 6298-6303, Oct. 2001.

[10] T. Kuo, H. Kim, D. M. Cannon, M. A. Shannon, J. V. Sweedler, and P. W. Bohn, "Nanocapillary arrays effect mixing and reaction in multilayer fluidic structures," Angewandte Chemie (International Ed. in English), vol. 43, pp. 1862-5, Mar. 2004.

[11] K. Fa, J. J. Tulock, J. V. Sweedler, and P. W. Bohn, "Profiling pH gradients across nanocapillary array membranes connecting microfluidic channels," Journal of the American Chemical Society, vol. 127, pp. 13928-13933, Oct. 2005.

[12] S. Dietrich, "New physical phases induced by confinement," Journal of Physics: Condensed Matter, vol. 10, no. 49, pp. 11469-11471, 1998.

[13] B. Zhao, J. S. Moore, and D. J. Beebe, "Principles of Surface-Directed liquid flow in microfluidic channels," Analytical Chemistry, vol. 74, no. 16, pp. 4259-4268, 2002.

[14] J. M. K. Ng, I. Gitlin, A. D. Stroock, and G. M. Whitesides, "Components for integrated poly(dimethylsiloxane) microfluidic systems," Electrophoresis, vol. 23, no. 20, pp. 3461-3473, 2002.

[15] G. M. Whitesides and A. D. Stroock, "Flexible methods for microfluidics.," Physics Today, vol. 54, p. 42, June 2001.

[16] G. M. Whitesides, "The origins and the future of microfluidics," Nature, vol. 442, pp. 368-373, July 2006.

[17] T. Young, "An essay on the cohesion of fluids," Philosophical Transactions of the Royal Society of London (1776-1886), vol. 95, no. 1, pp. 65-87, 1805.

[18] P. G. de Gennes, "Wetting: statics and dynamics," Reviews of Modern Physics, vol. 57, p. 827, July 1985.

[19] J. N. Israelachvili, Intermolecular And Surface Forces. Academic Press, 3 ed., 2003.

[20] A. W. Adamson, Physical Chemistry of Surfaces. Wiley \& Sons, 0006 ed., Sept. 1997.

[21] H. Hamaker, "The London-van der Waals attraction between spherical particles," Physica, vol. 4, pp. 1058-1072, Oct. 1937. 
[22] E. M. Lifshitz Soviet Phys. JETP, vol. 2, no. 73, 1956.

[23] A. Sharma, "Relationship of thin film stability and morphology to macroscopic parameters of wetting in the apolar and polar systems," Langmuir, vol. 9, pp. 861869, Mar. 1993.

[24] A. Sharma and A. T. Jameel, "Nonlinear stability, rupture, and morphological phase separation of thin fluid films on apolar and polar substrates," Journal of Colloid and Interface Science, vol. 161, pp. 190-208, Nov. 1993.

[25] F. B. Wyart and J. Daillant, "Drying of solids wetted by thin liquid films," Canadian Journal of Physics, vol. 68, p. 1084, 1990.

[26] A. Vrij, "Possible mechanism for the spontaneous rupture of thin, free liquid films," Discussions of the Faraday Society, vol. 42, pp. 23-33, 1966.

[27] E. Ruckenstein and R. K. Jain, "Spontaneous rupture of thin liquid films," Journal of the Chemical Society, Faraday Transactions 2, vol. 70, pp. 132-147, 1974.

[28] M. B. Williams and S. H. Davis, "Nonlinear theory of film rupture," Journal of Colloid and Interface Science, vol. 90, pp. 220-228, Nov. 1982.

[29] A. N. Frumkin, "On the wetting phenomena and attachment of bubbles," J. Phys. Chem., vol. 12, no. 4, pp. 337-345, 1938.

[30] C. L. M. H. Navier, "Memoire sur les lois du mouvement des fluides," Mem. Acad. Sci. Inst. France, vol. 6, pp. 389-440, 1822.

[31] G. G. Stokes, "On the theories of the internal friction of fluids in motion and of the equilibrium and motion of elastic solids," Trans. Camb. Philos. Soc., vol. 8, pp. 287-319, 1845 .

[32] H. Lamb, Hydrodynamics. Dover Publications, 6 ed., Nov. 1993.

[33] F. Brochard and P. G. D. Gennes, "Shear-dependent slippage at a polymer/solid interface," Langmuir, vol. 8, pp. 3033-3037, Dec. 1992.

[34] C. Neto, D. R. Evans, E. Bonaccurso, H. Butt, and V. S. J. Craig, "Boundary slip in Newtonian liquids: a review of experimental studies," Reports on Progress in Physics, vol. 68, no. 12, pp. 2859-2897, 2005. 
[35] R. Fetzer, M. Rauscher, A. Münch, B. A. Wagner, and K. Jacobs, "Slip-controlled thin-film dynamics," EPL (Europhysics Letters), vol. 75, no. 4, pp. 638-644, 2006.

[36] R. Seemann, M. Brinkmann, E. J. Kramer, F. F. Lange, and R. Lipowsky, "Wetting morphologies at microstructured surfaces," Proceedings of the National Academy of Sciences of the United States of America, vol. 102, pp. 1848-1852, Feb. 2005.

[37] K. Khare, M. Brinkmann, B. M. Law, E. L. Gurevich, S. Herminghaus, and R. Seemann, "Dewetting of liquid filaments in wedge-shaped grooves," Langmuir, vol. 23, pp. 12138-41, Nov. 2007.

[38] K. Khare, S. Herminghaus, J. Baret, B. M. Law, M. Brinkmann, and R. Seemann, "Switching liquid morphologies on linear grooves," Langmuir, vol. 23, pp. 1299713006, Dec. 2007.

[39] M. Brinkmann and R. Blossey, "Blobs, channels and cigars: Morphologies of liquids at a step," The European Physical Journal E: Soft Matter and Biological Physics, vol. 14, pp. 79-89, May 2004.

[40] S. Herminghaus, M. Brinkmann, and R. Seemann, "Wetting and dewetting of complex surface geometries," Annual Review of Materials Research, vol. 38, pp. 101-121, July 2008.

[41] F. W. Billmeyer, Jr., Textbook of Polymer Science. John Wiley \& Sons, 3rd ed., Mar. 1984.

[42] R. A. Jones, Soft Condensed Matter. Oxford University Press, USA, 1 ed., Aug. 2002.

[43] G. S. Fulcher, "Analysis of recent measurements of the viscosity of glasses," Journal of the American Ceramic Society, vol. 8, no. 6, pp. 339-355, 1925.

[44] A. K. Varshneya, Fundamentals of Inorganic Glasses. Academic Press, Nov. 1993.

[45] J. Fox and P. J. Flory, "Second-Order transition temperatures and related properties of polystyrene. I. influence of molecular weight," Journal of Applied Physics, vol. 21, pp. 581-591, June 1950. 
[46] E. Guth, H. M. James, and H. Mark, "The kinetic theory of rubber elasticity," Advances in Colloid Science, vol. 2, pp. 253-299, 1946.

[47] S. J. Clarson and J. A. Semlyen, Siloxane Polymers. Prentice Hall, 1993.

[48] C. M. Stafford, C. Harrison, K. L. Beers, A. Karim, E. J. Amis, M. R. VanLandingham, H. Kim, W. Volksen, R. D. Miller, and E. E. Simonyi, "A buckling-based metrology for measuring the elastic moduli of polymeric thin films," Nat. Mater., vol. 3, no. 8, pp. 545-550, 2004.

[49] O. Scott, M. Begley, U. Komaragiri, and T. Mackin, "Indentation of freestanding circular elastomer films using spherical indenters," Acta Materialia, vol. 52, pp. 4877-4885, Sept. 2004.

[50] J. Becker, G. Grün, R. Seemann, and K. Jacobs, "Dynamical pattern formation upon dewetting," cond-mat/0106313, June 2001.

[51] R. Fetzer, M. Rauscher, R. Seemann, K. Jacobs, and K. Mecke, "Thermal noise influences fluid flow in thin films during spinodal dewetting," Physical Review Letters, vol. 99, p. 114503, Sept. 2007.

[52] R. Konnur, K. Kargupta, and A. Sharma, "Instability and morphology of thin liquid films on chemically heterogeneous substrates," Physical Review Letters, vol. 84, no. 5, p. 931, 2000.

[53] R. Fetzer, Impact of different interfaces on the flow properties of complex fluids. $\mathrm{PhD}$ thesis, University of Saarland, 2006.

[54] J. Sagiv, "Organized monolayers by adsorption. 1. formation and structure of oleophobic mixed monolayers on solid surfaces," Journal of the American Chemical Society, vol. 102, no. 1, pp. 92-98, 1980.

[55] J. P. Cleveland, B. Anczykowski, A. E. Schmid, and V. B. Elings, "Energy dissipation in tapping-mode atomic force microscopy," Applied Physics Letters, vol. 72, pp. 2613-2615, May 1998.

[56] R. Hillenbrand, M. Stark, and R. Guckenberger, "Higher-harmonics generation in tapping-mode atomic-force microscopy: Insights into the tip-sample interaction," Applied Physics Letters, vol. 76, pp. 3478-3480, June 2000. 
[57] R. W. Stark and W. M. Heckl, "Fourier transformed atomic force microscopy: tapping mode atomic force microscopy beyond the Hookian approximation," Surface Science, vol. 457, pp. 219-228, June 2000.

[58] S. Crittenden, A. Raman, and R. Reifenberger, "Probing attractive forces at the nanoscale using higher-harmonic dynamic force microscopy," Physical Review B, vol. 72, p. 235422, Dec. 2005.

[59] J. Legleiter, M. Park, B. Cusick, and T. Kowalewski, "Scanning probe acceleration microscopy (SPAM) in fluids: Mapping mechanical properties of surfaces at the nanoscale," Proceedings of the National Academy of Sciences of the United States of America, vol. 103, pp. 4813-4818, Mar. 2006.

[60] S. Hembacher, F. J. Giessibl, and J. Mannhart, "Force microscopy with Light-Atom probes," Science, vol. 305, pp. 380-383, July 2004.

[61] M. Stark, R. W. Stark, W. M. Heckl, and R. Guckenberger, "Inverting dynamic force microscopy: From signals to time-resolved interaction forces," Proceedings of the National Academy of Sciences of the United States of America, vol. 99, pp. 84738478, June 2002.

[62] R. W. Stark, "Optical lever detection in higher eigenmode dynamic atomic force microscopy," Review of Scientific Instruments, vol. 75, pp. 5053-5055, Nov. 2004.

[63] R. Pericet-Càmara, A. Best, H. Butt, and E. Bonaccurso, "Effect of capillary pressure and surface tension on the deformation of elastic surfaces by sessile liquid microdrops: An experimental investigation," Langmuir, vol. 24, pp. 10565-10568, Oct. 2008.

[64] "Even though the glue does not contain solvents, a slight swelling of the pdms cannot be excluded. in any case, even though the substrate swelling would definitely not be a desired phenomenon, it would not directly disturb our imaging, as this is done on glassy PS, which is not affected by the swelling of the PDMS.."

[65] Y. Zhao, X. Shi, and W. Li, "Effect of work of adhesion on nanoindentation," Rev.Adv.Mater.Sci., vol. 5, pp. 348-353, 2003. 
[66] H. Hertz, "Über die berührung fester elastischer Körper," J. Reine Angew. Math., vol. 92, pp. 156-171, 1881.

[67] A. Rusanov, "Theory of the wetting of elastically deformed bodies," Colloid Journal, vol. 37, pp. 614-618, 1975.

[68] R. Seemann, S. Herminghaus, and K. Jacobs, "Shape of a liquid front upon dewetting," Physical Review Letters, vol. 87, p. 196101, Nov. 2001.

[69] F. Brochard-Wyart, P. de Gennes, H. Hervert, and C. Redon, "Wetting and slippage of polymer melts on semi-ideal surfaces," Langmuir, vol. 10, pp. 1566-1572, May 1994.

[70] C. Redon, J. B. Brzoska, and F. Brochard-Wyart, "Dewetting and slippage of microscopic polymer films," Macromolecules, vol. 27, pp. 468-471, Mar. 1994.

[71] C. Neto and K. Jacobs, "Dynamics of hole growth in dewetting polystyrene films," Physica A: Statistical Mechanics and its Applications, vol. 339, pp. 66-71, Aug. 2004.

[72] R. Fetzer, K. Jacobs, A. Münch, B. Wagner, and T. P. Witelski, "New slip regimes and the shape of dewetting thin liquid films," Physical Review Letters, vol. 95, p. 127801, Sept. 2005.

[73] A. Carré and M. Shanahan, "Viscoelastic braking of a running drop," Langmuir, vol. 17, pp. 2982-2985, May 2001.

[74] M. E. R. Shanahan, "The spreading dynamics of a liquid drop on a viscoelastic solid," Journal of Physics D: Applied Physics, vol. 21, no. 6, pp. 981-985, 1988.

[75] N. Zaghzi, A. Carré, M. E. R. Shanahan, E. Papirer, and J. Schultz, "A study of spontaneous rubber/metal adhesion. I. the rolling cylinder test," Journal of Polymer Science Part B: Polymer Physics, vol. 25, no. 11, pp. 2393-2402, 1987.

[76] F. B. Wyart, P. Martin, and C. Redon, "Liquid/liquid dewetting," Langmuir, vol. 9, pp. 3682-3690, Dec. 1993.

[77] P. Martin, A. Buguin, and F. Brochard-Wyart, "Bursting of a liquid film on a liquid substrate," EPL (Europhysics Letters), vol. 28, no. 6, pp. 421-426, 1994. 
[78] P. Lambooy, K. C. Phelan, O. Haugg, and G. Krausch, "Dewetting at the liquidliquid interface," Physical Review Letters, vol. 76, p. 1110, Feb. 1996.

[79] S. Qu, Dewetting dynamics at polymer-polymer interfaces. PhD thesis, State University of New York at Stony Brook, 1997. Ph.D.

[80] S. Daniel, M. K. Chaudhury, and J. C. Chen, "Fast drop movements resulting from the phase change on a gradient surface," Science, vol. 291, no. 5504, pp. 633-636, 2001.

[81] R. A. Hayes and B. J. Feenstra, "Video-speed electronic paper based on electrowetting," Nature, vol. 425, no. 6956, pp. 383-385, 2003.

[82] B. Zhao, J. S. Moore, and D. J. Beebe, "Surface-Directed liquid flow inside microchannels," Science, vol. 291, pp. 1023-1026, Feb. 2001.

[83] J. Z. Wang, Z. H. Zheng, H. W. Li, W. T. S. Huck, and H. Sirringhaus, "Dewetting of conducting polymer inkjet droplets on patterned surfaces," Nat Mater, vol. 3, pp. 171-176, Mar. 2004.

[84] D. E. Kataoka and S. M. Troian, "Patterning liquid flow on the microscopic scale," Nature, vol. 402, pp. 794-797, Dec. 1999.

[85] M. Brinkmann and R. Lipowsky, "Wetting morphologies on substrates with striped surface domains," Journal of Applied Physics, vol. 92, pp. 4296-4306, Oct. 2002.

[86] C. Rascon and A. O. Parry, "Geometry-dominated fluid adsorption on sculpted solid substrates," Nature, vol. 407, pp. 986-989, Oct. 2000.

[87] F. Mugele, A. Klingner, J. Buehrle, D. Steinhauser, and S. Herminghaus, "Electrowetting: a convenient way to switchable wettability patterns," Journal of Physics: Condensed Matter, vol. 17, no. 9, pp. S559-S576, 2005.

[88] K. Khare, M. Brinkmann, B. M. Law, S. Herminghaus, and R. Seemann, "Switching wetting morphologies in triangular grooves," The European Physical Journal Special Topics, vol. 166, no. 1, pp. 151-154, 2009.

[89] J. Boussinesq, Application des potentiels à l' étude de l'équilibre et du mouvement des solides élastiques. Gauthier Villard, Paris, 1885. 
[90] A. Pototsky, M. Bestehorn, D. Merkt, and U. Thiele, "Alternative pathways of dewetting for a thin liquid two-layer film," Phys. Rev. E, vol. 70, no. 2, 2004. 



\section{Acknowledgments}

"There is a crack in everything That's how the light gets in."

Leonard Cohen

The accomplishment of this thesis would not have been possible without the guidance, contribution and support from many different people.

I would like to thank Prof. Dr. S. Herminghaus for having given me the opportunity to conduct my research in the Department of Dynamics of Complex Fluids of the MaxPlanck Institute for Dynamics and Self-Organization, a leading Institute in the field of Physics of Complex Systems.

Special thanks go to my supervisor Prof. Dr. R. Seemann. I thank him for the trust showed in my person by accepting me in his group and for the time dedicated to me as well as to the other members of our group. With his strong background and expertise in this scientific field, he has always provided me with the right solution to any problem encountered during my thesis work. I learned a lot during this time and I am convinced that this knowledge will help me in the future.

I thank Dr. M. Brinkmann, Prof. Dr. A. Münch, Dr. B. Wagner and Dr. D. Peschka for interesting discussions.

It was a pleasure for me to be surrounded by all the wonderful people in our department. During my stay here I got help in many different ways from Monika, who did more than her ordinary secretary duties, including house-hunting, to help all the foreign students in the department, $U d o$, who has always been super-efficient in resolving any technical problems encountered in the lab and Semra who is a "fierce and mighty" lab technician and "the sweetest thing" at the same time! 
Krish, Tuul and Shashi, thank you for having been such great office-mates. I had lots of fun with you guys during our long hours in the lab and in the office. You have been great coffee- and tea-companions and I will surely miss our conversations once I am away. I wish you all the best for the future and hope that our paths will meet again some day. Sravanti, even though we did not have the chance to spend as much time together as I would have liked, you will always be in my thoughts. There have been several common dreams and hopes connecting us and I am sure we will see them fulfilled one day!

I thank Ludo and Dominik, my dear friends from the Univeristy of Saarbrücken. Ludo, conferences and workshops were much more fun when you were around! I still laugh at the time when we got kicked out of the locked swimming pool! Dominik, you have been a great "student". But more than that you have been a wonderful friend. I am very proud of you and I am sure you will do great whatever you decide to do in your life, because you put your heart in it. Keep doing your own thing...

A big thank you goes to the first friends I met here in Göttingen. Oliver, even for such a short time, you have been a great flatmate! Lukas, who would have thought that after meeting in Rome, we would still meet in Göttingen?! It is indeed a smal world! Jola, I will always remember the fun we had during our German classes, but even more after class!

My most sincere and warm-hearted thoughts go to my family. I am grateful to my parents, Panagiotis and Dimitra, for all they have taught me, but most of all I thank them for having raised me with the conviction and the courage to always follow my heart and my dreams. I thank my Italian parents Guido and Rosetta, my theia Noula and Pappou Thymios for being such a wonderful family to me. The warmth when I am around you has always soothed my heart and made my days brighter.

Words will never be enough to thank you, Daniel, for all that you have sacrificed these years in order to be by my side. I will forever be grateful to you for the love, support and strength you have constantly given me and for all the tears you have kissed away during my moments of disbelief. 


\section{Curriculum Vitae}

\section{Personal Information}

Name: $\quad$ Konstantina Kostourou

Date of Birth: 16 July 1979

Place of Birth: Athens, Greece

Address: $\quad$ Baumschulenweg 1, 37083 Göttingen

Nationality: Greek

\section{Education}

February 2006 - Ph.D. Candidate at the Max-Planck Institute for Dynamics present und Self-organization, Göttingen (Referee: Prof. Dr. Stephan Herminghaus, Co-referee: Prof. Dr. Christoph Schmidt, Supervisor: Prof. Dr. Ralf Seemann).

$2004-2005$

Research Assistant in the working group of Prof. Dr. Franco Decker, Chemistry Department, University of Rome "La Sapienza", Italy.

$2003-2004$ II level University Master, National Institute of Nuclear Physics, Rome, Italy. (Supervisors: Prof. Dr. Annibale Mottana and Dr. Augusto Marcelli).

1997 - 2003 Ptychion (Diploma) in Physics, Faculty of Physics, National and Kapodistrian University of Athens, Greece. (Supervisor: Prof. Dr. Manos Danezis)

High School Leaving Cerificate, $60^{\text {th }}$ Lyceum of Kypseli, Athens, Greece. 

